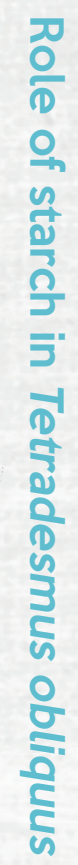

\title{
Role of starch in Tetradesmus obliquus
}

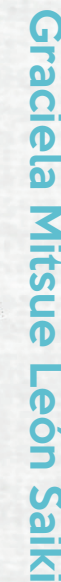




\section{Propositions}

1. Blocking starch production disturbs timing of cell division. (this thesis)

2. Diurnal light/dark cycles provide a benefit in microalgae. (this thesis)

3. Protocols that need a special touch are not good protocols.

4. A bioreactor is a dynamic system.

5. Taco shells do not make real tacos.

6. Cell phones are the new cigarettes.

Propositions belonging to the thesis entitled:

"Role of starch in Tetradesmus obliquus"

Graciela Mitsue León Saiki

Wageningen, 27 August 2018 


\section{Role of starch in \\ Tetradesmus obliquus}

Graciela Mitsue León Saiki 


\section{Thesis committee}

\section{Promotor}

Prof. Dr R.H. Wijffels

Professor of Bioprocess Engineering

Wageningen University \& Research

\section{Co-promotors}

Dr D.E. Martens

Associate professor, Bioprocess Engineering Group

Wageningen University \& Research

Dr D. van der Veen

Post-doc, Bioprocess Engineering Group

Wageningen University \& Research

\section{Other members}

Prof. Dr E.J. Smid, Wageningen University \& Research

Prof. Dr M.J.E.C. van der Maarel, University of Groningen

Dr B.O. Arredondo Vega, CIBNOR, La Paz, Mexico

Dr L. de Jaeger, Chr Hansen A/S, Hoersholm, Denmark

This research was conducted under the auspices of the Graduate School VLAG (Advanced studies in Food Technology, Agrobiotechnology, Nutrition and Health Sciences). 


\section{Role of starch in \\ Tetradesmus obliquus}

\section{Graciela Mitsue León Saiki}

Thesis

submitted in fulfilment of the requirements for the degree of doctor at Wageningen University

by the authority of the Rector Magnificus,

Prof. Dr A.P.J. Mol,

in the presence of the

Thesis Committee appointed by the Academic Board

to be defended in public

on Monday 27 August 2018

at 1:30 p.m. in the Aula. 
Graciela Mitsue León Saiki

Role of starch in Tetradesmus obliquus,

222 pages.

PhD thesis, Wageningen University, Wageningen, the Netherlands (2018)

With references, with summaries in English and Spanish

ISBN: 978-94-6343-308-2

DOI: https://doi.org/10.18174/454224 
Para Katzuo

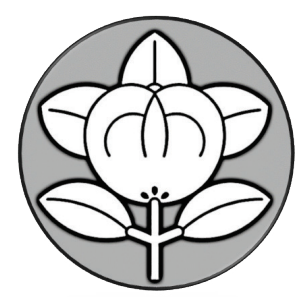




\section{Contents}

Chapter 1

Chapter 2

Chapter 3

Chapter 4

Chapter 5

Chapter 6

Chapter 7

References

Introduction and thesis outline

9

The role of starch as transient energy buffer in

19 synchronized microalgal growth in Tetradesmus obliquus

The diurnal transcriptional landscape of the 43 microalga Tetradesmus obliquus

The impact of day length on cell division and 81 efficiency of light use in a starchless mutant of Tetradesmus obliquus

Diurnal biochemical responses of Tetradesmus obliquus under light/dark cycles and nitrogen limitation

Metabolic modelling and energy parameter 119 estimation of Tetradesmus obliquus

General Discussion

Acknowledgements/agradecimientos

About the author 


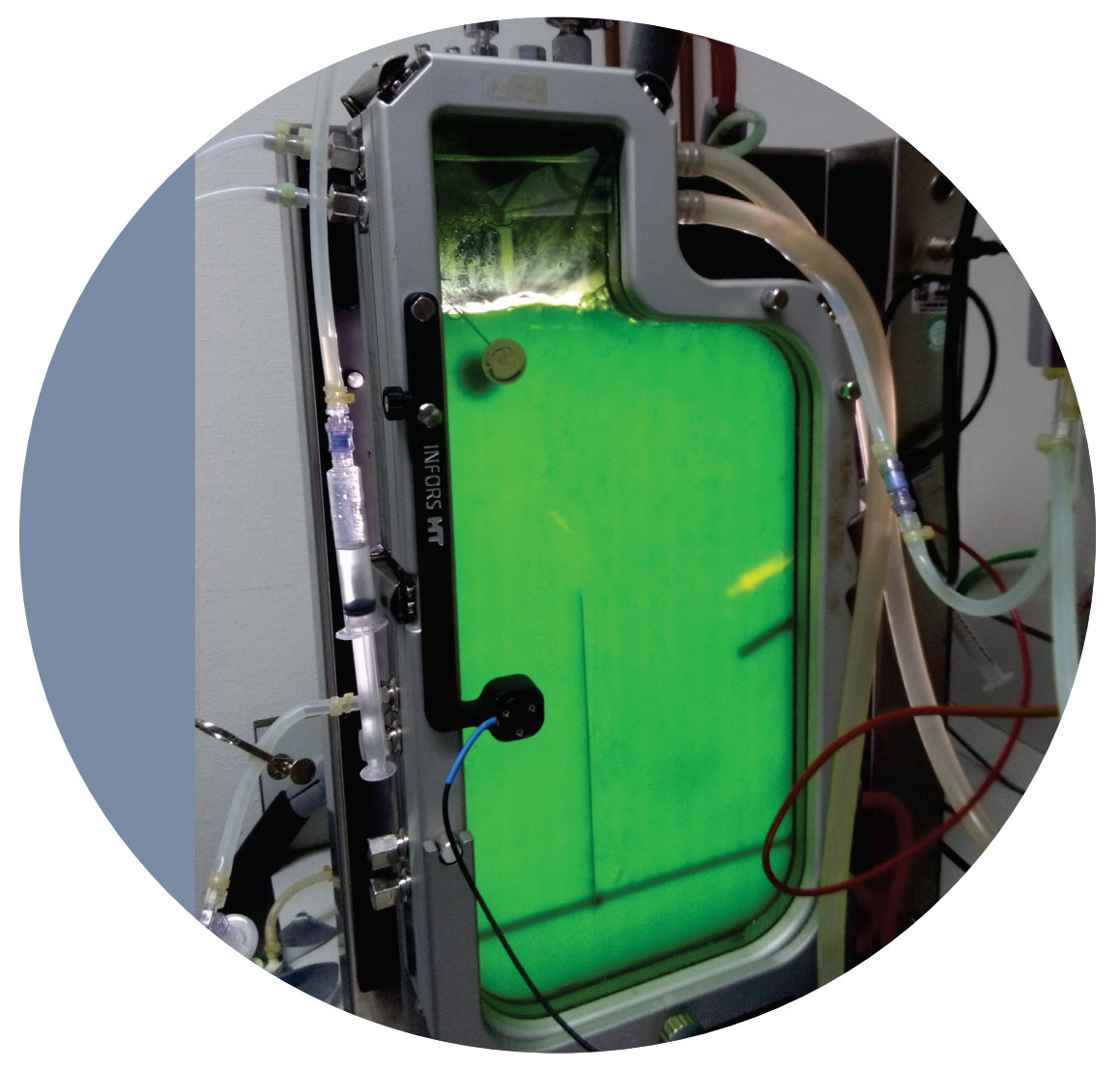




\section{Chapter 1}

Introduction and thesis outline 


\subsection{Microalgae production under light/dark cycles}

To reduce the dependence on fossil fuels, new sustainable biobased feedstocks need to be utilized (Ruiz et al., 2016). Microalgae are considered one of the most promising feedstocks as they can convert carbon dioxide not only into biofuels, but also into foods, feeds, and high value compounds. Microalgae are of special interest due to their capacity to produce carbon-rich lipids. Oleaginous microalgae can accumulate between $20-60 \%$ of triacylglycerides (TAG) on a dry weight basis under stress conditions (mainly nitrogen starvation). They can reach a higher areal productivity compared to agricultural crops without competing for arable land (Chisti, 2007; Hu et al., 2008; Wijffels and Barbosa, 2010). Additional to TAG, many microalgae produce starch under nitrogen starvation.

Large scale microalgal production will be done outdoors (Blanken et al., 2013), where microalgae are exposed to day/night (LD) cycles. Therefore an optimal production process must take into account the LD cycles during the growth phase (nutrient replete conditions) and the production phase (nutrient deplete conditions, e.g. nitrogen starvation).

Many organisms have organized their metabolism around the LD cycles and anticipate the changes in the environment, of which light availability is of special importance for microalgae (Shearman et al., 2000; Mittag, 2001; McClung, 2006; Salichos and Rokas, 2010; Causton et al., 2015). Environmental cues (e.g. sunrise and sunset) function as time indicators to entrain an organism's internal timing to a period of $24 \mathrm{~h}$ (McClung, 2006; Niwa et al., 2013). Photosynthetic microorganisms have evolved their metabolism in such a way as to capture sunlight efficiently during the day and to perform light sensitive processes (such as DNA replication and cell division) at night (Nikaido and Johnson, 2000; Suzuki and Johnson, 2001; Fábregas et al., 2002; de Winter et al., 2013). This is reflected by changes in cell size and biomass composition, mainly due to the accumulation of starch and cell division, during the diurnal cycle (Figure 1.1).

The advantage of synchronizing their metabolism to LD cycles becomes noticeable when comparing growth and energy conversion efficiency under LD cycles to those under continuous light (Jacob-Lopes et al., 2009; de Winter et al., 2013; Krzemińska et al., 2014). Under continuous light the cues for synchronisation do not work because 
the signal is always present. This results in asynchronous cultures where, at each moment, cells of all different stages of the LD cycle are present. This results in lower energy efficiencies of light use and lower biomass yield on light, demonstrating the benefit of synchronising metabolism to LD cycles.
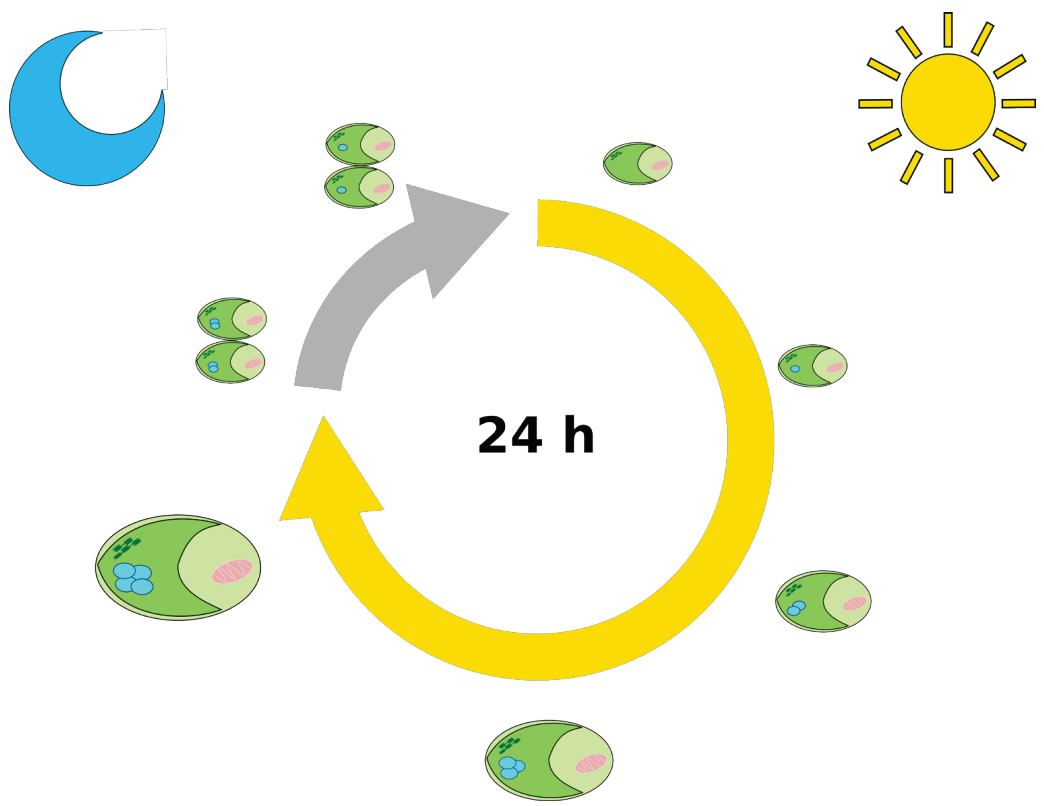

Figure 1.1. Representation of oscillations in cell size and biomass composition during light/dark cycles. Blue circles inside the cells represent starch.

\subsection{Starch in microalgae}

Many microalgae rely on starch under nitrogen replete conditions and LD cycles. When looking through the diurnal cycle (Figure 1.1), starch is accumulated during the light period, reaching the maximum content before the dark period starts, and it is consumed during the dark period to provide carbon precursors and energy for different metabolic processes (such as cell division).

In microalgae, other storage compounds besides starch can be used to store the captured light energy as chemical energy. An overview of the preferred storage compound for different microalgae is shown in Table 1.1. 
Table 1.1. Overview of preferred storage compound for different microalgae.

\begin{tabular}{|c|c|c|c|}
\hline Microalgae & Nitrogen replete & $\begin{array}{c}\text { Stress } \\
\text { conditions }\end{array}$ & Reference \\
\hline Tetradesmus obliquus & Starch & Starch and TAG & Remmers et al. (2017) \\
\hline T. obliquus s/m1 & - & TAG & $\begin{array}{c}\text { León-Saiki et al. (2017), } \\
\text { Remmers et al. (2017) }\end{array}$ \\
\hline $\begin{array}{l}\text { Neochloris } \\
\text { oleoabundans }\end{array}$ & Starch & Starch and TAG & $\begin{array}{l}\text { Breuer et al. (2012), } \\
\text { de Winter et al. (2013; } \\
\text { 2014) }\end{array}$ \\
\hline $\begin{array}{l}\text { Nannochloropsis } \\
\text { gaditana }\end{array}$ & Lipids and $\mathrm{CHO}$ & TAG & $\begin{array}{l}\text { Fábregas et al. (2002), } \\
\text { Simionato et al.(2013) }\end{array}$ \\
\hline $\begin{array}{l}\text { Nannochloropsis } \\
\text { oceanica }\end{array}$ & $\begin{array}{l}\text { TAG and CHO } \\
\text { (laminarin) }\end{array}$ & Lipids & $\begin{array}{c}\text { Meng et al. (2015), Poliner } \\
\text { et al. (2015), Banerjee et al. } \\
\text { (2017) }\end{array}$ \\
\hline $\begin{array}{l}\text { Chlamydomonas } \\
\text { reinhardtii }\end{array}$ & Starch & Starch and TAG & $\begin{array}{l}\text { Li et al. (2010a), } \\
\text { Willamme et al. (2015) }\end{array}$ \\
\hline C. reinhardtii BAFJ5 & - & TAG & Li et al. (2010a) \\
\hline $\begin{array}{l}\text { Phaeodactylum } \\
\text { tricornutum }\end{array}$ & $\begin{array}{l}\text { Lipids and water } \\
\text { soluble glucans } \\
\text { (chrysolaminarin) }\end{array}$ & TAG & $\begin{array}{l}\text { Breuer et al. (2012), } \\
\text { Chauton et al. (2013) }\end{array}$ \\
\hline Isochrysis sp. & $\begin{array}{l}\mathrm{CHO} \text { and neutral } \\
\text { lipids }\end{array}$ & $\begin{array}{l}\mathrm{CHO} \text { and neutral } \\
\text { lipids }\end{array}$ & Lacour et al. (2012) \\
\hline Chlorella vulgaris & Starch & Starch and TAG & $\begin{array}{c}\text { Brányiková et al. (2011), } \\
\text { Breuer et al. (2012) }\end{array}$ \\
\hline
\end{tabular}

TAG: triacylglycerides; CHO: Carbohydrates.

Overall, the role of starch in microalgae has not been explored as much as in plants (Smith and Stitt, 2007; Sulpice et al., 2009). In microalgae, starch is often considered as an unwanted product competing for carbon and energy with TAG (de Jaeger et al., 2014). As starch and TAG are competing for carbon precursors, a successful strategy to improve the TAG yield on light during nitrogen starvation is to knock out the ability to make starch, resulting in starchless mutants (Li et al., 2010a; de Jaeger et al., 2014).

When looking at microalgae starchless mutants, the behaviour is strain dependent. For example, the starchless mutant BAFJ5 of Chlamydomonas reinhardtii showed reduced growth compared to its wild-type under continuous light (Li et al., 2010a). In contrast, Vonlanthen et al. (2015) found no significant difference in growth for the starchless mutant ST68 of Chlorella sorokiniana compared to the wild-type under continuous light.

In summary, knocking out of the ability to make starch results in improved TAG yield on light during the production phase under nitrogen starvation. However, this knock 
out also results in reduced growth and energy conversion efficiency during the growth phase (nitrogen replete conditions), counteracting the gain in TAG productivity during the production phase. Therefore, a better understanding on the role of starch in oleaginous microalgae and how their metabolism is organized around LD cycles could provide information on how to improve the TAG yield on light without the drawbacks in energy conversion efficiency observed in starchless mutants.

\subsection{Aim and thesis outline}

This thesis aims to obtain more insight into the diurnal behaviour of the oleaginous microalgae Tetradesmus obliquus (Figure 1.2A) and the role of starch in this microalgae under light/dark (LD) cycles. The potential of this microalga for lipid production has been previously demonstrated (Mandal and Mallick, 2009; Breuer et al., 2012; Ho et al., 2012; Remmers et al., 2017). In addition, de Jaeger et al. (2014) developed a starchless mutant, s/m1. This starchless mutant cannot produce starch due to a single nucleotide polymorphism in the small subunit of ADP-glucose pyrophosphorylase, the committed step of starch biosynthesis (this is the synthesis of ADP-glucose from DGlucose-1-phosphate and ATP) (de Jaeger, 2015). This mutant showed an improvement of $51 \%$ in the maximum TAG yield on light compared to the wild-type ( $0.217 \mathrm{~g} \cdot \mathrm{mol}_{\mathrm{ph}}{ }^{-1}$ compared to $0.144 \mathrm{~g} \cdot \mathrm{mol}_{\mathrm{ph}}{ }^{-1}$ for its wild-type) and a higher maximum TAG content $\left(0.57 \mathrm{~g} \cdot \mathrm{g}_{\mathrm{DW}}{ }^{-1}\right.$ compared to $\left.0.45 \mathrm{~g} \cdot \mathrm{g}_{\mathrm{DW}}{ }^{-1}\right)$ in batch cultures under nitrogen starvation (Breuer et al., 2014).

To study the role of starch, first we compared the overall performance of the wild-type and the mutant using photobioreactors operated under continuous turbidostat mode (Figure 1.2B). This experimental set-up allows for a constant photon absorption rate as the culture was diluted with fresh medium when the light intensity at the rear of the reactor dropped below the setpoint. After reaching steady-state, the diurnal cycle was repeated every $24 \mathrm{~h}$ and, therefore, we could study the oscillations throughout the cycle.

We first examined the role of starch under nitrogen replete conditions in Chapter 2. We looked into the changes in the scheduling of cellular processes in T. obliquus wildtype and the starchless mutant under both continuous light and a 16:8 h light/dark (LD) cycle, especially in the trends in growth rate and timing of cell division. Furthermore, 
we analysed the changes in biomass composition in intervals of $1 \mathrm{~h}$ for the 16:8 $\mathrm{h}$ LD cycle and of $3 \mathrm{~h}$ for continuous light. Thus we showed the benefit of the ability to make starch under LD cycles and the impact on growth and cell division when starch synthesis is blocked.
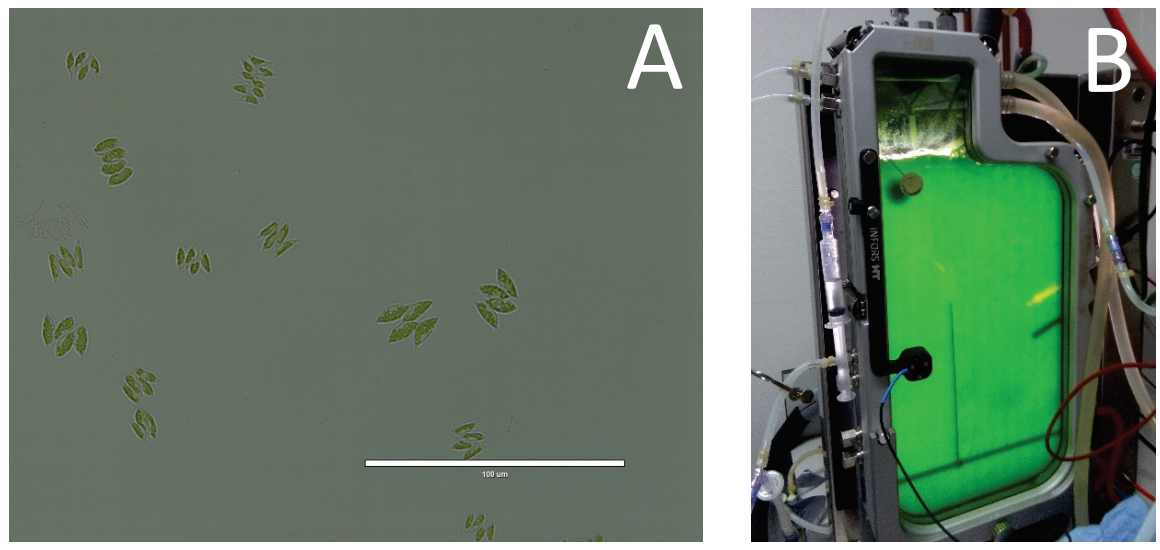

Figure 1.2. Microscopic photograph of Tetradesmus obliquus wild-type (A). The scale bar indicates $100 \mu \mathrm{m}$. Photograph of the flat panel photobioreactor used in this thesis for cultivation (B).

Then in Chapter $\mathbf{3}$ we looked into the regulation of cellular processes. We studied the diurnal changes in transcriptome of $T$. obliquus wild-type and starchless mutant $\operatorname{sim} 1$ under 16:8 $\mathrm{h}$ LD cycles. The annotation for the earlier sequenced genome (Carreres et al., 2017) was done and we looked into the diurnal effects in gene expression in intervals of $1 \mathrm{~h}$ for the wild-type. For the s/m1 mutant, gene expression was studied in intervals of $3 \mathrm{~h}$ and compared to the wild-type as a reference.

When thinking about microalgae outdoor production, variations in the length of the day and night periods will occur naturally. As this might impact he biomass productivity and biomass yield on light, in Chapter 4 we looked into the effect of three different LD cycles (12:12 h, 14:10 h, and 16:8 h LD) on T. obliquus wild-type and starchless mutant slm1. We looked at growth, timing of cell division, biomass yield on light and energy 
conversion efficiency. This way we could assess the importance of starch for longer dark periods.

Microalgae are of interest due to their high triacylglycerides (TAG) content, which is enhanced under stress conditions such as nitrogen limitation/starvation (Hu et al., 2008). Since large scale TAG production occurs outdoor under natural LD cycles, in Chapter 5 we looked into the diurnal changes of both T. obliquus wild-type and $s / m 1$ under nitrogen limitation. We looked into changes in growth and biomass composition, as well as the ability of both strains to accumulate and use TAG and starch during the $24 \mathrm{~h}$ cycle.

Next, we looked into metabolism in silico by developing a model describing the primary metabolism of T. obliquus in Chapter 6. This model included 351 reactions and 183 metabolites distributed over 4 compartments: cytosol, chloroplast, mitochondrion, and extracellular space. The model was completed by experimentally estimating the energy parameters for biomass formation and maintenance. Finally, the prediction capacity of the model was evaluated by looking into the biomass, TAG, and starch yield on light.

In Chapter 7 we discuss the role of starch during the growth and the production phase. First, the behaviour of oleaginous microalgae under both nitrogen replete and deplete conditions is discussed. Followed by our conclusions on the drawbacks from the lack of starch, by looking into the starchless mutant s/m1. Finally, the bottlenecks in microalgae metabolism are discussed and suggestions are made on how to expand our knowledge in microalgal metabolism. Altogether, this thesis shows that starch has an important role in the diurnal behaviour of the microalga Tetradesmus obliquus under light/dark cycles. 


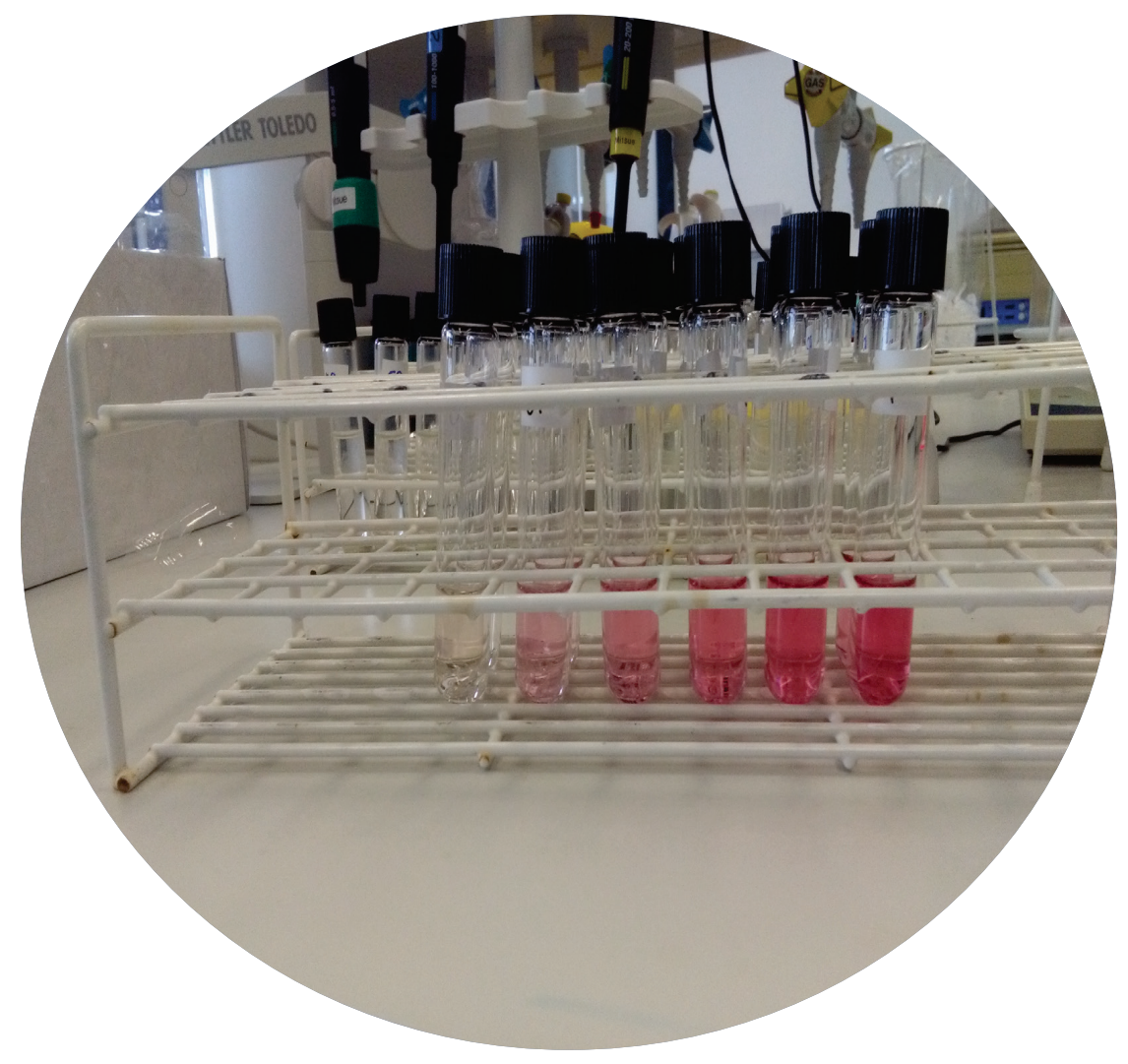




\section{Chapter}

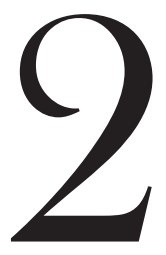

\section{The role of starch as transient energy buffer in synchronized microalgal growth in Tetradesmus obliquus}

This chapter is published as:

GM León-Saiki*, IM Remmers*, DE Martens, PP Lamers, RH Wijffels, D van der Veen(2017) The role of starch as transient energy buffer in synchronized microalgal growth in Acutodesmus obliquus. Algal Research 25, 160-167

* Authors contributed equally 
Photosynthetic organisms have evolved to use light efficiently by scheduling their cellular processes, such as growth and cell division, at specific times of the day. During the day, fixated carbon is used for growth and is partially stored as carbohydrates (e.g. starch). It is commonly assumed that this accumulated starch is essential for fuelling up cell division at night. To test this hypothesis, this study investigates growth, cell division, and presence of a transitory energy storage (TES) in both the wild-type and starchless mutant strain of Tetradesmus obliquus under light/dark (LD) cycles and nitrogen replete conditions.

T. obliquus (formerly known as Scenedesmus obliquus) wild-type utilized light $20 \%$ more efficiently under LD regimes compared with continuous light. When exposed to LD regimes, the wild-type scheduled cell division in a 4-hour period starting $2 \mathrm{~h}$ before 'sunset'. Starch acted as major transitory energy storage (TES) compound: it was accumulated during the last part of the light period and was consumed throughout the entire dark period. The s/m1 mutant, with a blocked starch synthesis pathway, showed diurnal rhythms in growth and cell division. However, no other carbohydrates nor triacylglycerols took over the role of TES compound in slm1. Therefore, in contrast to what is generally acknowledged, this study shows that neither starch nor any other major alternative TES is required for synchronized growth and cell division in T. obliquus. The starchless mutant did show a reduced growth and cell division rate compared to the wild-type. Starch thus plays a major role in efficient harnessing of light energy over LD cycles, likely because the ability to accumulate starch enhances biomass production capacity and accelerates cell division rate in $T$. obliquus. 


\subsection{Introduction}

Diurnal cycles of biological activities are ubiquitous and allow many organisms to anticipate and adapt to changing environmental conditions (McClung, 2006; Farré, 2012; Goldbeter et al., 2012; de Winter et al., 2013). In plants, it has previously been reported that numerous endogenous and environmental factors can regulate cell growth, with the light period being the major agent for entrainment of the diurnal cycle (Bishop and Senger, 1971; Graf et al., 2010; de Winter et al., 2013). Nowadays, microalgae receive attention as promising sustainable sources for both commodity products (e.g. biofuels and proteins) and high value compounds (e.g. polyunsaturated fatty acids and pigments). Although some microalgae-derived high-value products are already commercially available, large scale production is hampered by high costs of production and downstream processing (Pulz and Gross, 2004; Spolaore et al., 2006; Wijffels and Barbosa, 2010; Ruiz et al., 2016). One key bottleneck in reducing these costs is to improve biomass growth and yield (Wijffels and Barbosa, 2010). In both terrestrial plants and microalgae, it has been shown that growth is influenced by the presence of a diurnal energy storage system like starch, and that a lack of such system often results in reduced growth rates (Caspar et al., 1985; Stitt and Zeeman, 2012). Therefore, a thorough understanding of the role of storage metabolites under diurnal, outdoor conditions might contribute to commercialization of large-scale microalgal production systems (de Winter, 2015).

In most photosynthetic organisms, carbon is fixated in storage compounds (e.g. carbohydrates or lipids) during the light period which are subsequently consumed during the dark period to support different nocturnal metabolic processes (Nikaido and Johnson, 2000; Fábregas et al., 2002; de Winter et al., 2013; Michels et al., 2014; Edmundson and Huesemann, 2015). Specifically for microalgae, some strains use starch as the primary storage metabolite (Klein, 1987; Ballin et al., 1988; Brányiková et al., 2011; de Winter et al., 2014; Vitova et al., 2015), while others use non-starch carbohydrates (e.g. chrysolaminarin) or lipids (Lacour et al., 2012; Chauton et al., 2013; Poliner et al., 2015). The presence of such transitory energy storage (TES) compounds allows efficient use of sunlight under diurnal light/dark (LD) conditions, as was shown for Neochloris oleoabundans (de Winter et al., 2013). The role of TES compounds and the regulation of the cell cycle have been studied extensively (Wanka et al., 1970; 
Schulze et al., 1991; Sulpice et al., 2009; Garz et al., 2012; Bišová and Zachleder, 2014; Vitova et al., 2015). Many studies imply that the energy required for synchronized cell division at night is supplied by TES compounds. However, the mechanisms for carbon partitioning with regard to TES remain poorly understood (Klein, 1987; Li et al., 2011; Merchant et al., 2012; Bišová and Zachleder, 2014; Jia et al., 2015; Ma et al., 2016).

In recent years, many microalgal strains have been suggested as promising candidates for the production of food, fuel, or chemicals (Rosenberg et al., 2008; Rodolfi et al., 2009; Mata et al., 2010; Wijffels and Barbosa, 2010). Among them, Tetradesmus obliquus (formerly known as Scenedesmus obliquus and Acutodesmus obliquus (Krienitz and Bock, 2012)) is considered as an industrially relevant strain for food and fuel production (Breuer et al., 2012). To further improve triacylglycerols (TAG) productivity in $T$. obliquus, a starchless mutant was created using random mutagenesis (de Jaeger et al., 2014). This mutant showed a 51\% higher TAG yield on light compared to the wild-type $\left(0.144 \pm 0.004\right.$ in the wild-type to $0.217 \pm 0.011 \mathrm{~g}_{\mathrm{TAG}} \cdot \mathrm{mol}_{\mathrm{ph}}{ }^{-1}$ in the starchless mutant) under batch wise nitrogen starvation, while maintaining its photosynthetic efficiency (Breuer et al., 2014), showing the potential of blocking the starch pathway for TAG production. This starchless mutant shows potential for large scale food and biofuel production. Similar mutations have been generated in other microalgal strains and have been studied intensively for biomass productivity, lipid content, carbon partitioning and photosynthetic efficiency (Ramazanov and Ramazanov, 2006; Siaut et al., 2007; Wang et al., 2009; Li et al., 2010a; Work et al., 2010; Vonlanthen et al., 2015; Sirikhachornkit et al., 2016). However, the impact of starch deficiency on synchronized growth and cell division in microalgae remains unknown.

This study aims to understand the role of starch in synchronized growth and cell division in T. obliquus wild-type under nitrogen replete and different diurnal light conditions (continuous and day/night). A starchless mutant of $T$. obliquus was used to explore the consequences of the absence of starch and if there are other compounds, such as TAG, that can serve as alternative TES under diurnal LD cycles and nitrogen replete conditions. 


\subsection{Materials and Methods}

\subsubsection{Strains, pre-culture conditions and cultivation medium}

Wild-type Tetradesmus obliquus UTEX 393 was obtained from the Culture Collection of 12 and Scenedesmus obliquus (Krienitz and Bock, 2012). The starchless mutant of T. obliquus (s/m1) was generated as described by de Jaeger et al. (2014). Liquid cultures were maintained in a culture chamber with shaker $\left(25^{\circ} \mathrm{C}, 100 \mathrm{rpm}\right.$, air in headspace, continuous illumination at $30-40 \mu \mathrm{mol} \cdot \mathrm{m}^{-2} \cdot \mathrm{s}^{-1}$ ) in $250 \mathrm{~mL}$ Erlenmeyer flasks containing $100 \mathrm{~mL}$ of filter sterilized (pore size $0.2 \mu \mathrm{m}$ ) defined medium, as described in (Breuer et al., 2013b). Prior to the start of the experiments, cultures were placed in a shake incubator operating at $23^{\circ} \mathrm{C}$ with a light intensity of $180 \mu \mathrm{mol} \cdot \mathrm{m}^{-2} \cdot \mathrm{s}^{-1}$, a $16: 8 \mathrm{~h}$ (light/dark, LD) block cycle and a headspace enriched with $2.5 \% \mathrm{CO}_{2}$ to reach the desired inoculation cell density.

\subsubsection{Reactor set-up and experimental conditions}

T. obliquus was continuously cultivated in an aseptic flat panel airlift-loop reactor with a $1.7 \mathrm{~L}$ working volume and a $0.02 \mathrm{~m}$ light path (Labfors $5 \mathrm{Lux}$, Infors HT, Switzerland). Cultures were continuously sparged with air containing $2 \% \mathrm{CO}_{2}$ at $1 \mathrm{~L} \cdot \mathrm{min}^{-1}$. The temperature was controlled at $27.5^{\circ} \mathrm{C}$ and the $\mathrm{pH}$ was maintained at $7.0 \pm 0.1$ by automatic supply of $5 \% \mathrm{v} / \mathrm{v} \mathrm{H}_{2} \mathrm{SO}_{4}$. Several drops of a sterile $1 \% \mathrm{v} / \mathrm{v}$ solution of antifoam (Antifoam B, Baker, The Netherlands) were manually added to the culture when foam was observed (0-1 $\left.\mathrm{mL} \cdot \mathrm{day}^{-1}\right)$. The reactors were illuminated by $260 \mathrm{LED}$ lamps with a warm white spectrum $(450-620 \mathrm{~nm})$ spread evenly on the reactor's culture side. A black cover was placed on the back of the reactor to ensure that environmental light could not enter the reactor. Before inoculation, the incident photon flux density was calibrated and set at $500 \mu \mathrm{mol} \cdot \mathrm{m}^{-2} \cdot \mathrm{s}^{-1}$. Light was provided either in continuous mode or in a 16:8 h LD block cycle. After inoculation, the light intensity at the back side of the culture was continuously measured by a light meter (LI-250, Licor, USA). All cultivations were turbidostat controlled, which ensured automatic dilution of the culture with fresh medium when the light intensity at the back of the reactor dropped below the setpoint $\left(10 \mu \mathrm{mol} \cdot \mathrm{m}^{-2} \cdot \mathrm{s}^{-1}\right)$. During all cultivations, cultures were exposed to light limitation only 
and nitrogen was continuously measured to ensure that there was no nitrogen limitation.

The reactor was inoculated to an optical density $\left(O D_{750}\right)$ of 0.1 . Duplicate independent experiments were run to check reproducibility. Systems were operated in batch mode until the light at the back of the reactor reached the setpoint. At this moment, the turbidostat control was started and the system was allowed to reach steady state. Cultures exposed to diurnal light conditions showed identical repetitive $24 \mathrm{~h}$ oscillations in dilution rate. Therefore, steady state was defined as a constant biomass concentration and dilution rate for a period of at least 3 residence times. The overflow was collected on ice for $24 \mathrm{~h}$ periods and used to determine the $24 \mathrm{~h}$ average dilution rate $\left(D_{24 h}\right.$ in day $\left.{ }^{-1}\right)$ and biomass composition according to de Winter et al. (2017a).

The total amount of light absorbed is kept constant by the turbidostat control. This means that changes in biomass concentration in the reactor are possible when light absorbing and scattering properties of the biomass change (e.g. due to cell division or changes in pigmentation) over short time intervals. Therefore, growth rate does not equal the dilution rate over small time intervals $\left(D_{t}\right.$ in $\left.h^{-1}\right)$ during the day. The timespecific cell division rate $\left(\mu_{\mathrm{t}}\right.$ in $\left.\mathrm{h}^{-1}\right)$ can be calculated using a balance for cell counts $\left(\mathrm{C}_{\text {cells }}\right.$ in number of cells $\left.\cdot \mathrm{mL}^{-1}\right)$ over short time intervals ( $\left.<15 \mathrm{~min}\right)$, as shown in Equation 2.1:

$$
\frac{d C_{c e l l s}}{d t}=-D_{t} \cdot C_{\text {cells }}+\mu_{t} \cdot C_{\text {cells }}
$$

Sampling was done every hour for the experiments with a LD cycle. For all the other experiments, sampling was done every $3 \mathrm{~h}$. Liquid samples were freshly taken from the reactor and either immediately used for wet analysis or centrifuged for $5 \mathrm{~min}$ at 1200 x g for biochemical analysis. For biochemical analysis, the resulting pellet was transferred to bead beating tubes (Lysing Matrix E; MP Biomedicals Europe) or glass tubes (for total carbohydrates analysis), freeze dried overnight and stored at $-20{ }^{\circ} \mathrm{C}$ until further analysis. 


\subsubsection{Wet biomass analysis: Biomass concentration}

Optical density was measured at $750 \mathrm{~nm}\left(O D_{750}\right)$. The dry weight (DW) concentration was determined by filtrating a known volume of culture broth over pre-weighted glass fibre filters (Whatman ${ }^{\mathrm{TM}}$, GE Healthcare, UK) and measuring the weight increase of the filters after drying, as described by Kliphuis et al. (2012).

\subsubsection{Biochemical analysis}

\section{Total carbohydrate and starch content}

Total carbohydrates were extracted and quantified according to Dubois et al (DuBois et al., 1956) and Herbert et al. (1971). A phenol-sulphuric acid solution was added to 5-10 mg freeze dried algae. The absorbance was measured at $483 \mathrm{~nm}$ using glucose monohydrate as standard. Samples were analysed in duplicate. Starch content was determined using a total starch kit (Megazyme, Ireland) with modifications as described by de Jaeger et al. (2014) with the difference that $5 \mathrm{mg}$ of freeze dried biomass was used for the analysis. A known amount of starch was analysed as positive control and glucose monohydrate was used as standard on each assay. Starch productivity was calculated using a balance for starch over short time intervals (Equation 2.2). With the change over one or three hours intervals in starch content $\left(\frac{d C_{\text {starch }}}{d t}\right)$, dilution rate $\left(D_{t}\right)$, starch content $\left(C_{\text {starch, }}\right)$ and the starch productivity $\left(r_{\text {starch, } t}\right)$ :

$$
\frac{d C_{\text {starch }}}{d t}=-D_{t} \cdot C_{\text {starch }, t}+r_{\text {starch }, t}
$$

\section{Lipid analysis}

Triacylglycerol (TAG) content, total fatty acid (TFA) content, and fatty acid composition were determined as described by Breuer et al. (2013a) with the following modifications. Lipids were extracted from $5 \mathrm{mg}$ of lyophilized biomass in the presence of a chloroform:methanol $(1: 1.25 \mathrm{v} / \mathrm{v})$ solution containing $180 \mu \mathrm{g} \cdot \mathrm{mL}^{-1}$ glyceryl trinonadecanoate (T4632; Sigma-Aldrich) and $300 \mu \mathrm{g} \cdot \mathrm{mL}^{-1}$ 1,2-dipentadecanoyl-snglycero-3-phospho-(1'-rac-glycerol) (sodium salt) (840446, Avanti Polar Lipids Inc.) as internal standards. Chloroform and methanol were evaporated and the lipid extract 
was dissolved in $1 \mathrm{~mL}$ hexane:diethylether (7:1 v/v). The lipid extract was separated in neutral and polar lipids using a Sep-Pak Vac silica cartridge (6 cc, $1 \mathrm{~g}$; Waters). Silica cartridges were prewashed with $10 \mathrm{~mL}$ of hexane before loading the sample onto the column. The neutral lipid fraction, which contains TAG, was eluted with $10 \mathrm{~mL}$ of hexane:diethylether $(7: 1 \mathrm{v} / \mathrm{v})$. The polar lipid fraction, which contains mainly membrane lipids, was eluted with $10 \mathrm{~mL}$ of methanol:acetone:hexane $(2: 2: 1 \mathrm{v} / \mathrm{v} / \mathrm{v})$. Both extracts were separately methylated and quantified using gas chromatography (GC-FID) as described by Breuer et al. (2013a). The TFA profile and content were calculated as the sum of each individual fatty acid in the neutral and polar lipid fraction.

\section{Protein}

The total protein concentration was determined using the colorimetric assay (Bio-Rad DC protein assay) as described by Postma et al. (2015) with the difference that 10 $15 \mathrm{mg}$ of freeze dried biomass was used for analysis.

\subsubsection{Cell number, diameter and division rate}

T. obliquus is characterized by the formation of coenobia (Bišová and Zachleder, 2014). Cells were separated, but not disintegrated, by sonication for $30 \mathrm{~s}$ at $30 \%$ amplitude using a probe sonicator (Sonics vibra-cell, USA). Single cell presence was verified under the microscope. Cell number and diameter were determined using a Beckman Coulter Multisizer 3 (Beckman Coulter Inc., USA). The sonicated culture was diluted 200 times in Isoton ${ }^{\circledR}$ II diluent solution. Duplicate manual cell counts were done using a Neubauer improved counting chamber (DHC-N01, C-Chip, INCYTO, Republic of Korea).

\subsubsection{Biomass yield on light}

Biomass yield on light $\left(\mathrm{Y}_{\mathrm{x}, \mathrm{ph}}\right.$ in $\left.\mathrm{g}_{\mathrm{DW}} \cdot \mathrm{mol}_{\mathrm{ph}}{ }^{-1}\right)$ was calculated using the daily dilution rate $\left(D_{24 h}\right.$ in day $\left.^{-1}\right)$, the biomass concentration in the overflow $\left(C_{x, 24 h}\right.$ in $\left.g \cdot L^{-1}\right)$ and the volumetric photon supply rate $\left(r_{\mathrm{ph}}\right.$ in $\left.\mathrm{mol}_{\mathrm{ph}} \cdot \mathrm{L}^{-1} \cdot \mathrm{day}^{-1}\right)$ using Equation 2.3:

$$
Y_{x, p h}=\frac{D_{24 h} \cdot C_{X}}{r_{p h}}
$$




\subsubsection{Reproducibility and statistical analysis}

Biological replicate $(n=2)$ cultivations were run to check reproducibility under all experimental conditions. Unless stated differently, biochemical analysis was performed with technical duplicates $(n=2)$. Student's T-test with a significance level of $p<0.05$ was used to support the results and conclusions in this study.

\subsection{Results and discussion}

\subsubsection{T. obliquus wild-type shows synchronized growth under LD conditions}

To investigate the role of starch as transitory energy storage (TES) mechanism in synchronized cell division in microalgae, we first characterized the growth of T. obliquus wild-type under 16h:8 h light/dark (LD) cycles. Duplicate reactors were subjected to LD cycles at an incident light intensity of $500 \mu \mathrm{mol} \cdot \mathrm{m}^{-2} \cdot \mathrm{s}^{-1}$. All cultures were maintained at a fixed level of absorbed light in turbidostat-operated reactors, as the outgoing light intensity was measured and controlled at a predetermined setpoint $\left(10 \mu \mathrm{mol} \cdot \mathrm{m}^{-2} \cdot \mathrm{s}^{-1}\right)$. During the light period, constant turbidity was maintained by automatically adding fresh media.

In steady state, the average biomass concentration over 24 hours is constant and the daily dilution rate is equal to the average specific growth rate over a repetitive $24 \mathrm{~h}$ LD period. T. obliquus wild-type exhibited an average dilution rate $\left(D_{24 h}\right)$ of $1.12 \pm 0.01$ day $^{-1}$.

The time specific dilution rate fluctuates clearly in a cyclic pattern (Figure 2.1A), when calculated over $15 \mathrm{~min}$ time intervals. The rate initially increases and reaches a maximum of $0.11 \mathrm{~h}^{-1}$ after $5 \mathrm{~h}$ of light. Thereafter it decreases to 0 when the dark period starts ( $16 \mathrm{~h}$ after 'sunrise'). This fluctuating dilution rate pattern repeats itself every $24 \mathrm{~h}$, indicating a synchronization to LD cycles. The cyclic pattern in dilution rate is due to variations in growth rate and light absorption (e.g. pigmentation or scattering due to alterations in biomass composition) (de Winter et al., 2014). The biomass concentration remained constant at $1.25 \pm 0.25 \mathrm{~g} \cdot \mathrm{L}^{-1}$ (Figure $2.1 \mathrm{~B}$ ) and, therefore, biomass productivity is proportional to the dilution rate. 

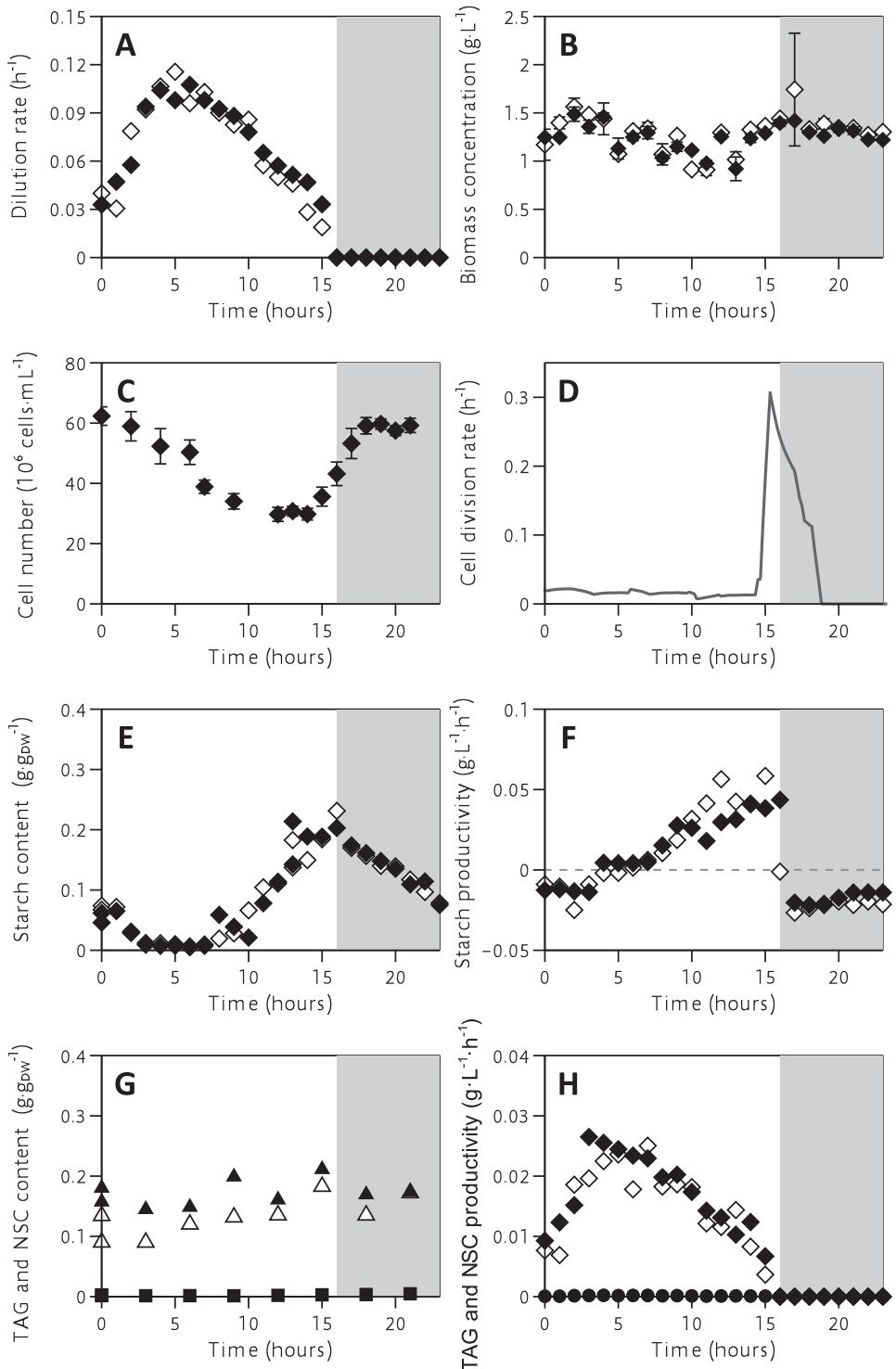

Figure 2.1. Daily variation in (A) dilution rate, (B) biomass concentration, (C) cell number, (D) cell division rate, (E) starch content, (F) starch productivity, (G) triacylglycerols (TAG, squares) and non-starch carbohydrates (NSC, triangles) content, and (H) TAG (circles) and NSC (diamonds) productivity for Tetradesmus obliquus wild-type under light/dark cycles. Open and closed symbols represent independent duplicate cultures. The $x$ axis shows hours after 'sunrise'. Shaded areas indicate the dark period. Error bars show the standard deviation on technical variability $(n=2)$. Error bars for starch, TAG and NSC are not shown because they were smaller than $3 \%$ compared to the average value. 
To explain the change in dilution pattern, we studied the changes in cell number and diameter throughout the diurnal cycle. During each cycle, the cell number doubled from $28.4 \pm 0.4$ million cells $\cdot \mathrm{mL}^{-1}$ to $61 \pm 2.8$ million cells $\cdot \mathrm{mL}^{-1}$ (Figure $2.1 \mathrm{C}$ ) during the last $2 \mathrm{~h}$ of light ( $14 \mathrm{~h}$ after 'sunrise') and the first $2 \mathrm{~h}$ of dark (16-17 hours after 'sunrise'). Total cell number remained stable afterwards until the end of the dark period. When the dark period was over, the culture was diluted and cells were washed out of the reactor during the first $12 \mathrm{~h}$ of light (Figure 2.1C).

By calculating the cell division rate (Figure 2.1D) based on dilution rates and cell numbers, we observed that the cell division rate increased sharply $2 \mathrm{~h}$ before the start of the dark period ( $14 \mathrm{~h}$ after 'sunrise') and decreased to zero approximately $2 \mathrm{~h}$ after the dark period started ( $18 \mathrm{~h}$ after 'sunrise'). In accordance with this, the cell diameter increased during the light period (from 3 to 14 hours after 'sunrise') and thereafter decreased (from 4.2 to $3.3 \mu \mathrm{m}$ ) due to cell division (Supplementary Figure S2.1). These results show that LD cycles result in synchronization of cell division in T. obliquus wildtype. Similar results have been described previously (Bongers, 1958; Senger, 1970). Bongers (1958) also found increasing cell volume during a $14 \mathrm{~h}$ light period with a simultaneous decrease in cell number for continuous cultures. When the dark period started, mature cells divided within $2 \mathrm{~h}$ into new cells and the cell diameter decreased to the original value.

\subsubsection{Biomass composition of T. obliquus wild-type fluctuates throughout diurnal light/dark cycles}

In order to further study the role of transitory energy storage (TES) compounds on synchronized cell division, we examined the diurnal changes in biomass composition in $1 \mathrm{~h}$ intervals. When T. obliquus was grown under diurnal LD cycles, it was observed that starch metabolism was tightly synchronized to LD cycles (Figure 2.1E). Starch was accumulated during the second half of the light period and consumed throughout the entire dark period. Starch started accumulating 7 hours after 'sunrise', simultaneously with a decrease in biomass productivity, and reached its maximum content of $0.20 \mathrm{~g} \cdot \mathrm{g}_{\mathrm{DW}}{ }^{-1}$ at the end of the light period. In the last hours of light, the change in biomass productivity was solely caused by starch accumulation. Therefore, the biomass productivity was equal to the starch productivity. Overnight losses in biomass 
and starch are similar (Supplementary Table S2.2). This supports the hypothesis that starch is respired overnight, as also observed in other diurnal studies on photosynthetic organisms (Blanken et al., 2017). Interestingly, although starch is consumed throughout the entire dark period, complete degradation was only observed $3 \mathrm{~h}$ after 'sunrise'.

Based on the diurnal accumulation and consumption, starch seems to act as the TES compound in T.obliquus wild-type. Previous work suggested that other storage metabolites such as triacylglycerols (TAG) or non-starch carbohydrates (NSC) could also act as TES (de Winter et al., 2014; Jia et al., 2015). However, these metabolite concentrations remained stable throughout the LD cycle (Figure 2.1G). The NSC fraction, which is defined as the total carbohydrate content minus the starch content, was stable at approximately $0.20 \mathrm{~g} \cdot \mathrm{gDw}^{-1}$. In addition, the TAG content was lower than $0.01 \mathrm{~g} \cdot \mathrm{g}_{\mathrm{DW}}{ }^{-1}$ and did not change throughout the diurnal cycle. As a consequence, both the NSC and TAG productivity followed the diurnal trend of biomass production rate (Figure $2.1 \mathrm{H}$ ). This observation suggests that starch acts as the sole TES compound, while the rate of NSC and TAG only fluctuates as a result of the production of functional biomass. We conclude that solely starch is used as the TES compound in T. obliquus wild-type.

\subsubsection{Continuous illumination abolishes synchronized growth in T. obliquus wild-type}

To further study the impact of LD cycles on cell physiology, we also cultivated T. obliquus wild-type under continuous light conditions. Here, as with the LD cycles, the biomass concentration remained stable due to turbidostat control (Supplementary Figure S2.2A). In contrast to the cultures exposed to LD cycles, no cyclic diurnal behaviour was observed in the cultures adapted to continuous light. All measured parameters were constant over time: total carbohydrates $0.33 \mathrm{~g} \mathrm{~g}_{\mathrm{DW}}{ }^{-1}$, proteins $0.47 \mathrm{~g}$ $\mathrm{gDW}^{-1}$, TAG $0.002 \mathrm{~g} \mathrm{gDw}^{-1}$ and a dilution rate of $1.54 \pm 0.03 \mathrm{day}^{-1}$. In addition, no apparent fluctuations in the biomass productivity and composition (protein, total carbohydrates, and TAG content) were observed (Figure S2.2E and G). This indicates that T. obliquus does not synchronize its cell division under constant light conditions. Similar observations have been reported in terrestrial plants, where transcripts involved in 
starch metabolism were tightly light regulated; when plants were placed in dark conditions the oscillations diminished almost immediately (Smith, 2004; Lu, 2005). In addition, no growth rhythms were found in maize (Zea mays), rice (Oryza sativa) (Poiré et al., 2010), and the microalga Neochloris oleoabundans (de Winter et al., 2013) when grown under constant light conditions.

\subsubsection{The starchless mutant T. obliquus slm1 shows synchronized growth}

It is often hypothesized that starch accumulation in plants acts as overflow metabolism during the light period and as a transitory carbon storage to fuel physiological processes (such as scheduled cell division) during the dark period (Caspar et al., 1985; Weise et al., 2011; de Winter et al., 2013; Webb and Satake, 2015). In T. obliquus wildtype, we showed that starch acts as the sole transitory energy storage (TES) molecule under nitrogen replete conditions (Figure 2.1E and G). The starch and TAG biosynthesis pathways are competing for carbon precursors (Fan et al., 2012). Thus, it was hypothesized that when the pathway to starch synthesis is blocked, the carbon and energy flux was automatically redirected towards TAG. As TAG could also be used as diurnal TES compound in other microalgae (Poliner et al., 2015), we hypothesized that these starchless mutants could potentially use TAG as the TES compound. To test this hypothesis, we examined growth characteristics of $T$. obliquus starchless mutant s/m1 (de Jaeger et al., 2014) under continuous light and diurnal light/dark (LD) cycles. S/m1 is not capable of producing starch due to a mutation in the small subunit of ADPglucose pyrophosphorylase (de Jaeger, 2015), a regulatory enzyme responsible for the first committed step in starch biosynthesis (i.e. synthesis of ADP-Glucose from ATP and D-Glucose-1-phosphate).

When s/m1 was subjected to a $16: 8 \mathrm{~h}$ LD cycle, an average dilution rate of $0.90 \pm 0.01$ day $^{-1}$ was found. Dilution rate patterns of $s / m 1$ were similar to those observed for the wild-type (Figure 2.2A) but absolute values were approximately $20 \%$ lower. As for the wild-type, biomass concentration remained constant over a $24 \mathrm{~h}$ cycle (Figure 2.2B); therefore, biomass productivity is proportional to the dilution rate. Also here, the biomass productivity of the $\operatorname{s} / \mathrm{m} 1$ was approximately $20 \%$ lower compared to the wild-type under LD cycles. 

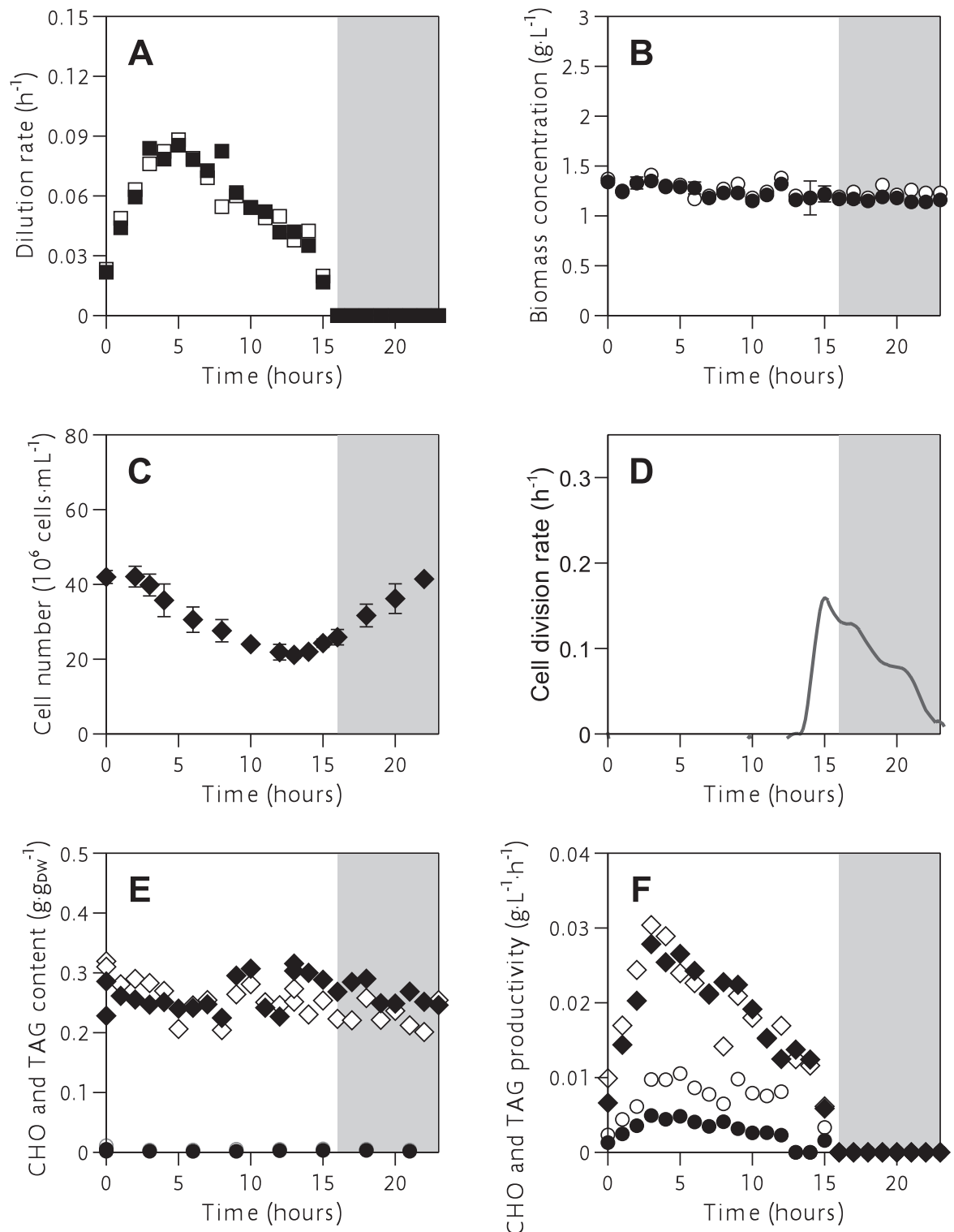

Figure 2.2. Daily variation in (A) dilution rate, (B) biomass concentration, (C) cell number, (D) cell division rate, $(\mathrm{E})$ carbohydrates (CHO, diamonds) and triacylglycerols (TAG, circle) content (E), and (F) CHO (diamond) and TAG (circle) productivity for T. obliquus slm1 under light/dark cycles. Open and closed symbols represent independent duplicate cultures. The $x$ axis indices hours after 'sunrise'. Shaded areas indicate the night period for the LD cultures. Error bars show the standard deviation of technical replicates $(n=2)$. 
As for the wild-type, the s/m1 was also grown under continuous light. However, under continuous light the dilution rate was even lower for $\operatorname{s} / m 1$ when compared to the wildtype $\left(1.03 \pm 0.06\right.$ day $^{-1}$ for s/m1 compared with $1.50 \pm 0.10$ day $^{-1}$ for the wild-type, Supplementary Figure S2.2D). Similar reduced growth rates were also observed for starchless mutants of Chlamydomonas reinhardtii (Li et al., 2010b).

To obtain insight into the scheduling of cell division and possible interaction with an alternative TES, we also studied cell diameter and division rate throughout a $24 \mathrm{~h} \mathrm{LD}$ cycle (with $16 \mathrm{~h}$ of light, followed by $8 \mathrm{~h}$ of darkness). As shown in Figure 2.2C and D, T. obliquus $\operatorname{sim} 1$ shows cyclic behaviour in cell division rate and cell diameter (data available in Figure S2.1) throughout the LD cycle. During each cycle, cell number doubled from $22.0 \pm 0.7$ million cells $\mathrm{mL}^{-1}$ to $41.4 \pm 0.5$ million cells $\cdot \mathrm{mL}^{-1}$ (Figure $2.2 \mathrm{C}$ ). Cell division started $2 \mathrm{~h}$ before the dark period ( $14 \mathrm{~h}$ after 'sunrise') as for the wildtype. However, while for the wild-type cell division was confined to a 4-hour period, the mutant continued cell division until the end of the dark period. Subsequently cell division stopped and the cell number decreased during the light period due to dilution of the culture. In accordance with this, average cell diameter increased from 3.8 to $5.1 \mu \mathrm{m}$ during the light period (Supplementary Figure S2.1) and decreased due to cell division afterwards (from $14 \mathrm{~h}$ after 'sunrise'). Interestingly, the mutant s/m1 and the wild-type show different subpopulation patterns in cell diameter. The wild-type shows three subpopulations, while the mutant is more uniform. We do not know the cause for this. However, it can be seen that in the case of the wild-type all three subpopulations increase in size during the day and decrease in size due to division in the night (Supplementary Figure S2.1). Based on our results, we can conclude that starch is not necessary for synchronized cell division. However, presence of starch seems to enhance the rate of cell division and seems to confine it to a shorter time period.

Many studies speculate on the need of a TES compound to support cell division and maintenance during the night. To determine if other energy dense molecules (TAG or non-starch carbohydrates) could act as TES, we also analysed the biomass composition with intervals of $1 \mathrm{~h}$. TAG content remained below the detection limit (Figure 2.2E). Although the non-starch carbohydrate content was slightly higher in the starchless mutant ( $22 \%$ versus $27 \%$ for the wild-type and starchless mutant, respectively), there was no diurnal pattern observed under either LD cycles or continuous light (Figure 2.2E 
and Supplementary Figure S2.2F). Therefore, in contrast to our expectations, these findings indicate that neither TAG nor any alternative carbohydrates (Figure 2.2E) are used in $\operatorname{s} / m 1$ to replace starch as TES agent.

\subsubsection{A starch knockdown reduces energy conversion efficiency}

During photosynthesis, light energy is converted into chemical energy. This energy is subsequently used to produce biomass. Under LD cycles, the presence of a TES, such as starch, can improve the light-to-biomass conversion efficiency during nitrogen replete growth (Klein, 1987; Lacour et al., 2012; de Winter et al., 2013; Vitova et al., 2015). To elaborate on this, we calculated the biomass yield on light (Eq. 2.3). T. obliquus wild-type uses light more efficiently under LD cycles compared to continuous light (Figure 2.3), as it produces approximately $20 \%$ more biomass per mole photon absorbed when grown under LD cycles $\left(0.98 \pm 0.00 \mathrm{~g} \cdot \mathrm{mol}_{\mathrm{ph}}{ }^{-1}\right.$ for continuous light compared to $1.18 \pm 0.03 \mathrm{~g} \cdot \mathrm{molph}^{-1}$ for LD cycles). In contrast, the $\mathrm{s} / \mathrm{m} 1$ showed similar biomass yields on light for both continuous and LD conditions $\left(0.72 \pm 0.04 \mathrm{~g} \cdot \mathrm{mol}_{\mathrm{ph}}{ }^{-1}\right.$ for continuous light compared to $0.80 \pm 0.04 \mathrm{~g} \cdot \mathrm{molph}^{-1}$ for LD cycles). As the biomass composition was different for the two strains under the different light regimes, we corrected for the actual energy harnessed in the biomass (Supplementary File S2.1). Similar trends as for biomass yield on light were found for the energy conversion efficiencies under the different light regimes (Supplementary Table S2.1). We can therefore conclude that $T$. obliquus wild-type is able to retain more of the supplied energy in biomass under LD cycles compared to continuous light conditions and less energy is dissipated.

The benefit observed for the wild-type under LD cycles as compared to continuous light is lost for the starchless mutant $\operatorname{s} / m 1$. We thus conclude that starch plays a role in efficient harnessing of light energy. Possibly, this is because the accumulation of starch allows the algae to fixate extra light energy during the day. In addition, starch could supply energy and carbon and electrons for processes at night that prepare the algae for efficient light harvesting, or reduce photo damage, at the start of the day. This statement is supported by the increased dilution rate of the wild-type immediately after switching on the light (Figure 2.1A).

Also, when comparing the two strains, we showed that the starchless mutant has 
reduced energy conversion efficiencies under both continuous and LD conditions compared to the wild-type (Supplementary Table S2.1) under nitrogen replete conditions. Interestingly, under nitrogen starvation and continuous light, Breuer et al. (2014) observed that the mutant $s / m 1$ has a similar energy conversion efficiency compared with the wild-type. Nitrogen starvation is often concomitant with arrest of cell growth. Apparently the capability to accumulate starch only gives an advantage with respect to energy efficiency in growing algae. More research is needed to develop a better understanding in role of starch and the limitations in metabolism under diurnal LD cycles. Eventually, this knowledge may support new strategies to overcome the reduced photosynthetic efficiencies in, for example, starchless mutants.

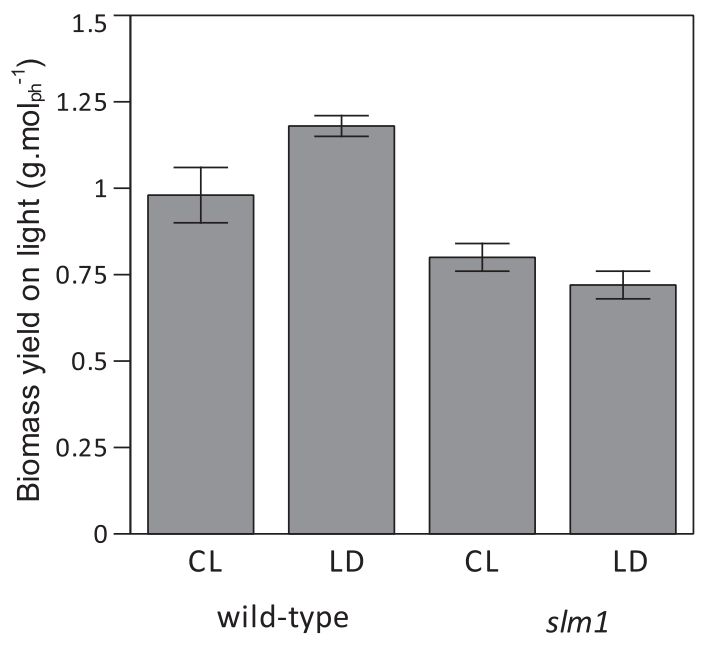

Figure 2.3. Biomass yield on light. Values represent averages of at least 3 daily overflows for two replicate cultivation runs. The error bars show the standard deviation. CL: continuous light, LD: Light/dark cycles. 


\subsection{Conclusions}

T. obliquus synchronizes to LD cycles. In this study we found diurnal cyclic behaviour in growth rate, cell division and starch content for T. obliquus wild-type. Our results show that starch acts as the only major transitory energy storage (TES) metabolite during LD cycles for T. obliquus. By accumulating starch during the day and respiring it during the night, the wild-type maximizes its overall light energy conversion efficiency.

When starch synthesis is blocked, as in the starchless mutant $\operatorname{sim1}$, we also observed diurnal rhythms in growth and cell division. Storage compounds, however, did not show diurnal changes. This indicates that none of the measured metabolites (TAG and carbohydrates) took over the role of starch as a TES compound. Transient storage of energy is therefore not required for synchronized cell division and growth. The starchless mutant did show a reduced growth rate compared to the wild-type, as well as an increased time necessary to perform cell division. It is therefore possible that the presence and nightly respiration/degradation of starch enhances the rate of cell division.

In addition, the lack of a TES resulted in a lower energy conversion efficiency compared to the wild-type. Therefore, by removing the pathway for starch synthesis, the benefit of LD cycles in energy conversion efficiency is lost. A deeper insight into the role of starch in light harnessing efficiency is required to fully benefit from the LD cycles.

\section{Funding}

This work was supported by the Consejo Nacional de Ciencia y Tecnología - CONACyT, Mexico, Scholar 218586/Scholarship 314173 and the EU FP7 Fuel4Me project under grant agreement No. 308983. In addition, GMLS. is part of the program "Doctores Jóvenes para el Desarrollo Estratégico Institucional" by the Universidad Autónoma de Sinaloa.

\section{Acknowledgments}

The authors would like to thank Snežana Gegić for her help with the carbohydrate analysis and Wendy Evers with the TAG analysis. 


\section{Supplementary information}
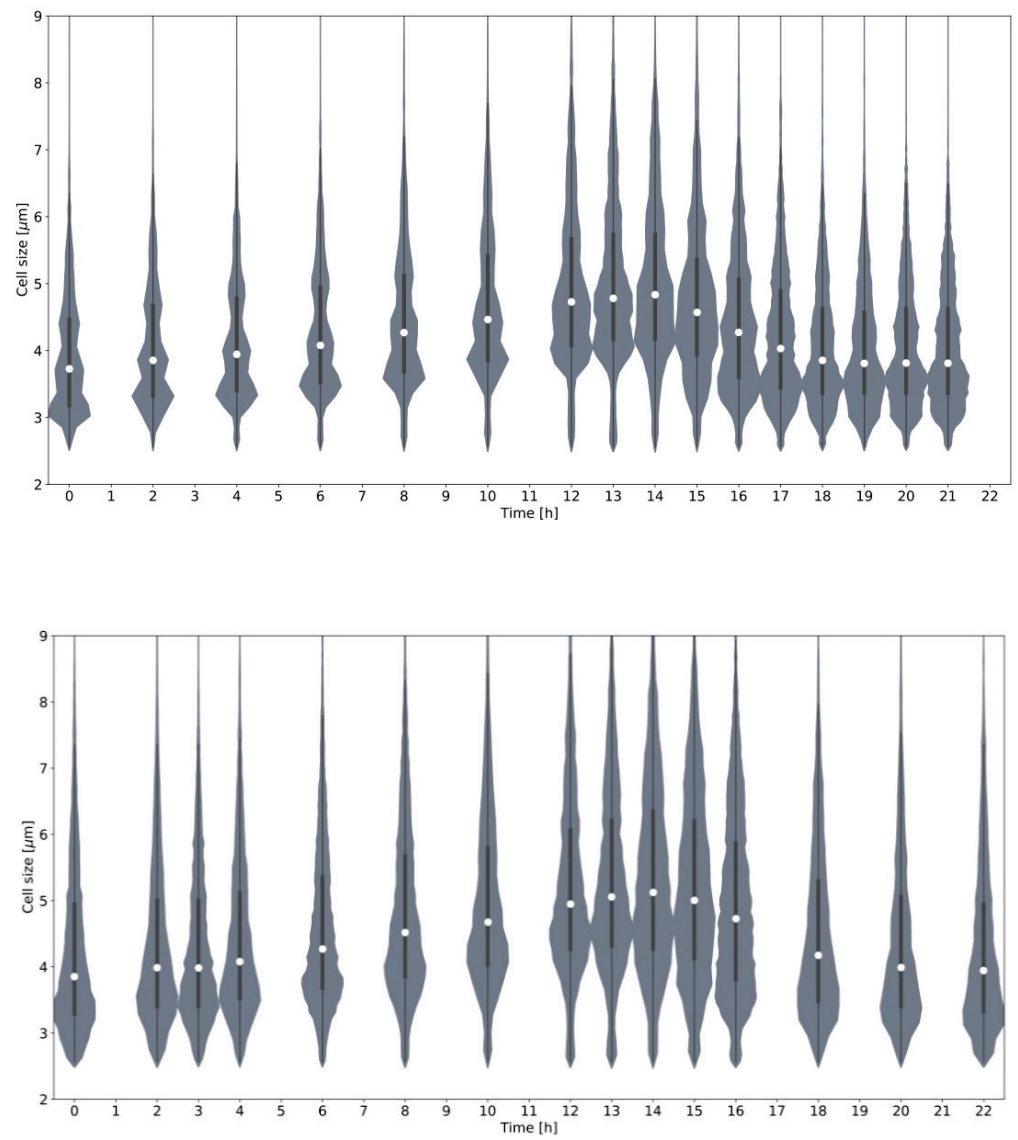

Supplementary Figure S2.1. Cell diameter distribution for Tetradesmus obliquus wildtype (top) and $s / m 1$ (bottom). The length of the diagram represent the size of the population, the width represents the density of the population on each size value, and the white dot represents the average. The $x$ axis shows hours after 'sunrise'. Dark period starts at $16 \mathrm{~h}$. 

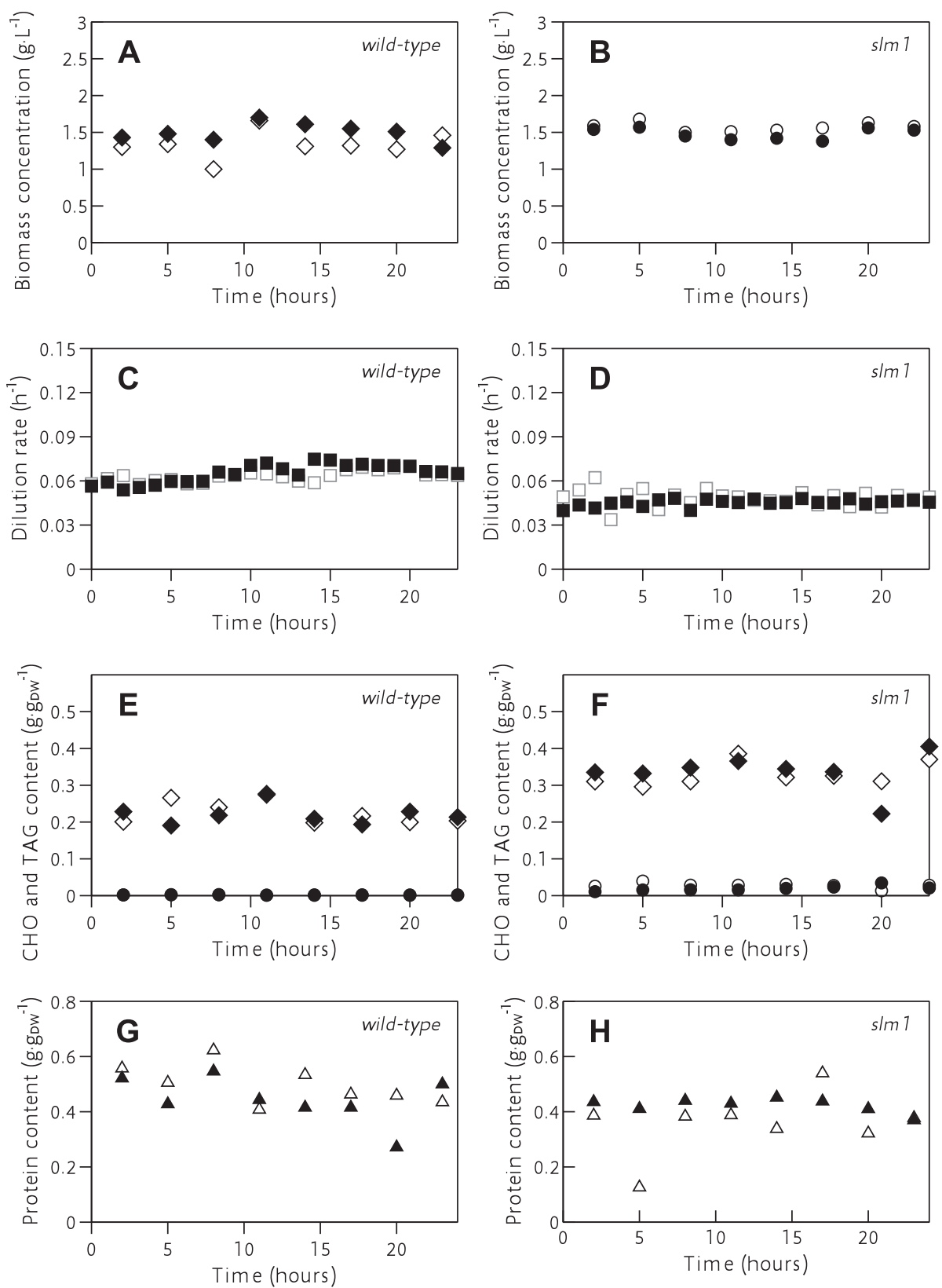

Supplementary Figure S2.2. Trends in biomass concentration (A and B), dilution rate ( $C$ and $D$ ), carbohydrate ( $\mathrm{CHO}$ in diamonds) and TAG (circles) ( $E$ and $\mathrm{F}$, and proteins ( $G$ and $H$ ) for Tetradesmus obliquus wild-type (A, C, E, G) and $\operatorname{sim1~(B,~D,~F,~H)~}$ under continuous light. Open and closed symbols represent duplicate cultures. 
Supplementary Table S2.1. Theoretical energy fixation rate for the wild-type and slm1 under continuous and LD regimes. Standard deviations (SD) are shown under average values (average \pm SD).

\begin{tabular}{|c|c|c|c|c|c|c|c|c|}
\hline \multirow{4}{*}{$\begin{array}{l}\text { Strain } \\
\text { Light cycle } \\
\text { Dry weight } \\
\text { concentration } \\
\left(\mathrm{g} \cdot \mathrm{L}^{-1}\right)\end{array}$} & \multicolumn{4}{|c|}{ Wild-type } & \multicolumn{4}{|c|}{$s / m 1$} \\
\hline & \multicolumn{2}{|c|}{ Continuous light } & \multicolumn{2}{|c|}{ LD cycles } & \multicolumn{2}{|c|}{$\begin{array}{c}\text { Continuous } \\
\text { light }\end{array}$} & \multicolumn{2}{|c|}{ LD cycles } \\
\hline & 1.38 & 1.34 & 1.42 & 1.40 & 1.64 & 1.53 & 1.17 & 1.15 \\
\hline & \pm 0.06 & \pm 0.05 & \pm 0.05 & \pm 0.03 & \pm 0.10 & \pm 0.03 & \pm 0.07 & \pm 0.08 \\
\hline Dilution rate & 1.52 & 1.49 & 1.14 & 1.14 & 0.97 & 1.07 & 0.84 & 0.84 \\
\hline$\left(\right.$ day $\left.^{-1}\right)$ & \pm 0.08 & \pm 0.14 & \pm 0.01 & \pm 0.02 & \pm 0.02 & \pm 0.04 & \pm 0.02 & \pm 0.01 \\
\hline $\begin{array}{l}\text { Triacylglycerides } \\
\text { content }\end{array}$ & 0.00 & 0.00 & 0.00 & 0.00 & 0.02 & 0.03 & 0.00 & 0.01 \\
\hline (g.gow) & \pm 0.00 & \pm 0.00 & \pm 0.00 & \pm 0.00 & \pm 0.00 & \pm 0.00 & \pm 0.00 & \pm 0.00 \\
\hline Carbohydrate content & 0.25 & 0.22 & 0.19 & 0.19 & 0.26 & 0.29 & 0.21 & 0.25 \\
\hline$\left(g \cdot g_{D w}\right)$ & \pm 0.03 & \pm 0.04 & \pm 0.01 & \pm 0.01 & \pm 0.02 & \pm 0.01 & \pm 0.02 & \pm 0.04 \\
\hline $\begin{array}{l}\text { Energy fixation rate } \\
\text { (mol photons } \mathrm{L}^{-1} \text { day }^{-1} \text { ) }\end{array}$ & 1.30 & 1.16 & 1.00 & 0.98 & 1.02 & 1.01 & 0.58 & 0.60 \\
\hline $\begin{array}{l}\text { Photon absorption } \\
\text { rate } \\
\text { (mol photons } \mathrm{L}^{-1} \text { day }^{-1} \text { ) }\end{array}$ & \multicolumn{2}{|c|}{2.05} & \multicolumn{2}{|c|}{1.36} & \multicolumn{2}{|c|}{2.05} & \multicolumn{2}{|c|}{1.36} \\
\hline $\begin{array}{l}\text { Energy fixation } \\
\text { efficiency }\end{array}$ & 63.35 & 56.80 & 73.72 & 72.34 & 49.79 & 49.30 & 42.63 & 43.99 \\
\hline (\% photons used) & \pm 0.01 & \pm 0.01 & \pm 0.00 & \pm 0.01 & \pm 0.04 & \pm 0.03 & \pm 0.00 & \pm 0.02 \\
\hline
\end{tabular}
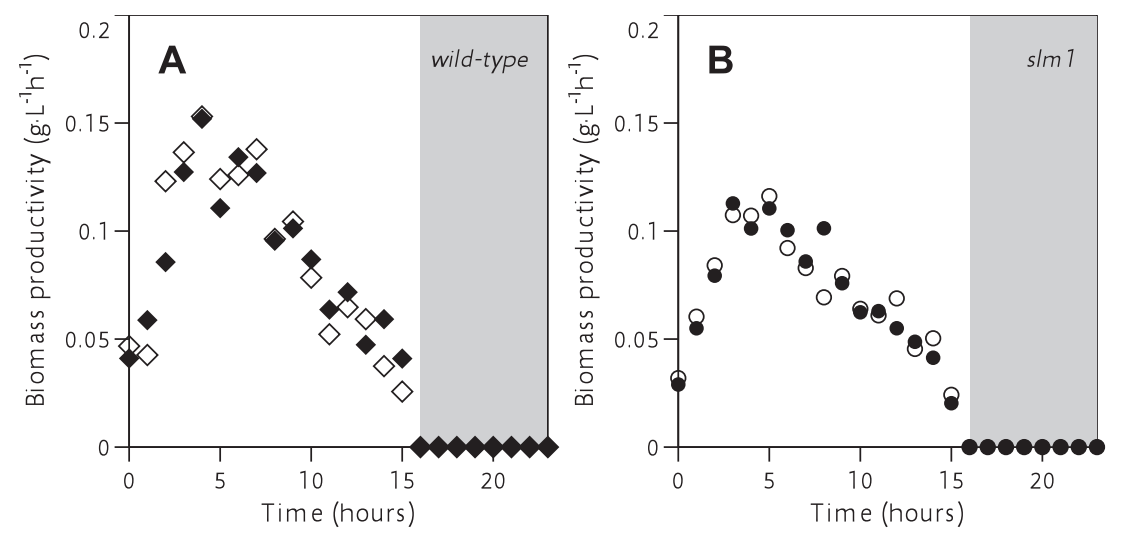

Supplementary Figure S2.3. Diurnal biomass productivity under LD cycles for Tetradesmus obliquus wild-type (A) and starchless mutant (B). Open and closed symbols represent duplicate cultures. The $x$ axis shows hours after 'sunrise'. Shaded areas indicate the dark period. 

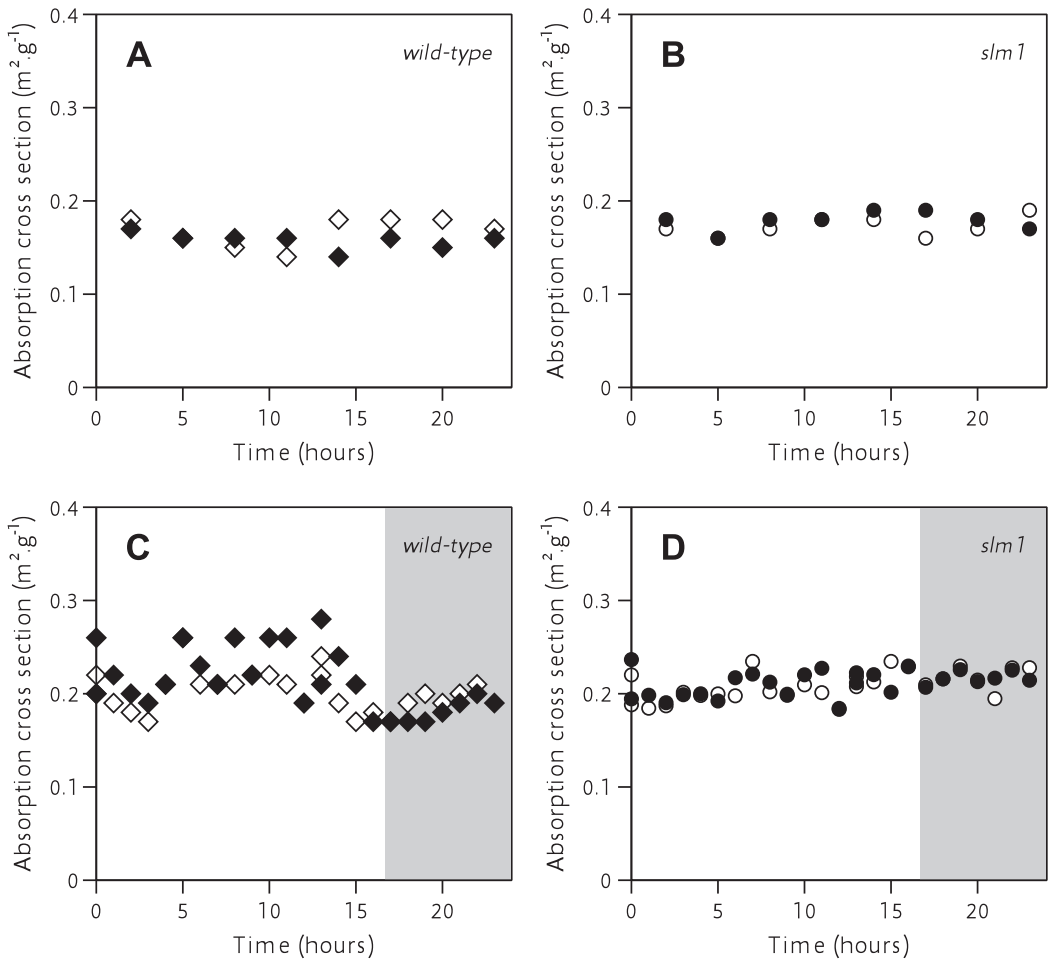

Supplementary Figure S2.4. Absorption cross section for continuous light (A and B) and light/dark cycles ( $C$ and $D$ ) for Tetradesmus obliquus wild-type ( $A$ and $C$ ) and starchless mutant slm1 (B and D). Open and closed symbols represent replicate cultures. The absorption spectrum was measured in diluted cell suspensions $\left(\mathrm{OD}_{750}\right.$ of approximately 0.5) according to de Mooij et al. (2016) Shaded areas indicate the night period for the light/dark cultures.

Supplementary Table S2.2. Overnight differences in dry weight and starch concentration. Standard deviations (SD) are shown under average values (average $\pm \mathrm{SD})$.

\begin{tabular}{lccc}
\hline Strain & \multicolumn{2}{c}{ Wild-type } & s/m1 \\
& $\begin{array}{c}\text { Dry weight } \\
\text { concentration } \\
\left(\mathbf{g} \cdot \mathrm{L}^{-1}\right)\end{array}$ & $\begin{array}{c}\text { Starch } \\
\left(\mathbf{g} \cdot \mathrm{L}^{-1}\right)\end{array}$ & $\begin{array}{c}\text { s/my weight } \\
\text { concentration } \\
\left(\mathbf{g} \cdot \mathrm{L}^{-1}\right)\end{array}$ \\
\hline Sunset & 1.43 & 0.26 & 1.20 \\
& \pm 0.02 & \pm 0.03 & \pm 0.00 \\
Sunrise & 1.24 & 0.08 & 1.24 \\
Overnight & \pm 0.00 & \pm 0.01 & \pm 0.02 \\
difference & 0.19 & 0.19 & -0.04 \\
\hline
\end{tabular}




\section{Supplementary File S2.1 - Energy conversion efficiency}

The theoretical energy conversion efficiency was calculated from the theoretical photon requirements for biomass production and the photon consumption rate, as illustrated by Equation S2.1. Biomass was considered to consist of TAG, starch and functional biomass. The functional biomass was determined by subtracting the measured fractions of TAG and starch from the measured total biomass. The photon requirements for functional biomass, TAG and starch were calculated based on the $24 \mathrm{~h}$ average productivity ( $\mathrm{r}_{\text {TAG }}$ for TAG productivity, $\mathrm{r}_{\text {starch }}$ for starch productivity and

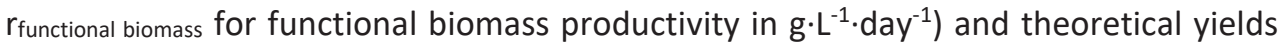
on photons of these biomass fractions. These theoretical yields were estimated using flux balance analysis according to (Kliphuis et al., 2012; Breuer et al., 2015a) and were $1.62 \mathrm{~g}$ functional biomass $\cdot \mathrm{mol}$ photon $^{-1}\left(\mathrm{Y}_{\text {max }}\right.$ functional biomass,ph), $3.24 \mathrm{~g}_{\text {starch }} \cdot \mathrm{mol}$ photon $^{-1}\left(\mathrm{Y}_{\text {max }}{ }_{\text {carbs,ph}}\right)$ and $1.33 \mathrm{~g}_{\mathrm{TAG}} \cdot \mathrm{mol}_{\text {photon }}{ }^{-1}\left(\mathrm{Y}_{\mathrm{max}}{ }_{\mathrm{TAG}, \mathrm{ph}}\right)$. The photon consumption rate $\left(r_{\mathrm{ph}}\right.$,absorbed in $\mathrm{mol} \mathrm{ph} \cdot \mathrm{L}^{-1} \cdot$ day $\left.^{-1}\right)$ was calculated by subtracting the incoming from the outgoing light intensity.

Theoretical energy conversion efficiency

$$
=\frac{\frac{r_{T A G}}{Y_{T A G, p h}^{\max }}+\frac{r_{\text {starch }}}{Y_{\text {starch }, p h}^{\max }}+\frac{r_{\text {fuctional biomass }}}{Y_{\text {functional biomass }, p h}^{\text {max }}}}{r_{\text {ph,absorbed }}}
$$

Wild-type cultures exposed to LD cycles used $73 \pm 1 \%$ of the photons supplied to create biomass, compared with $60 \pm 5 \%$ when grown under continuous light conditions. In contrast to the wild-type, the efficiency was lower for the starchless mutant $s / m 1$ under LD cycles compared with continuous light $(43 \pm 1 \%$ under LD cycles compared with $50 \pm 0.4 \%$ under continuous light regime, $T$-test, $p<0.05$ ). 


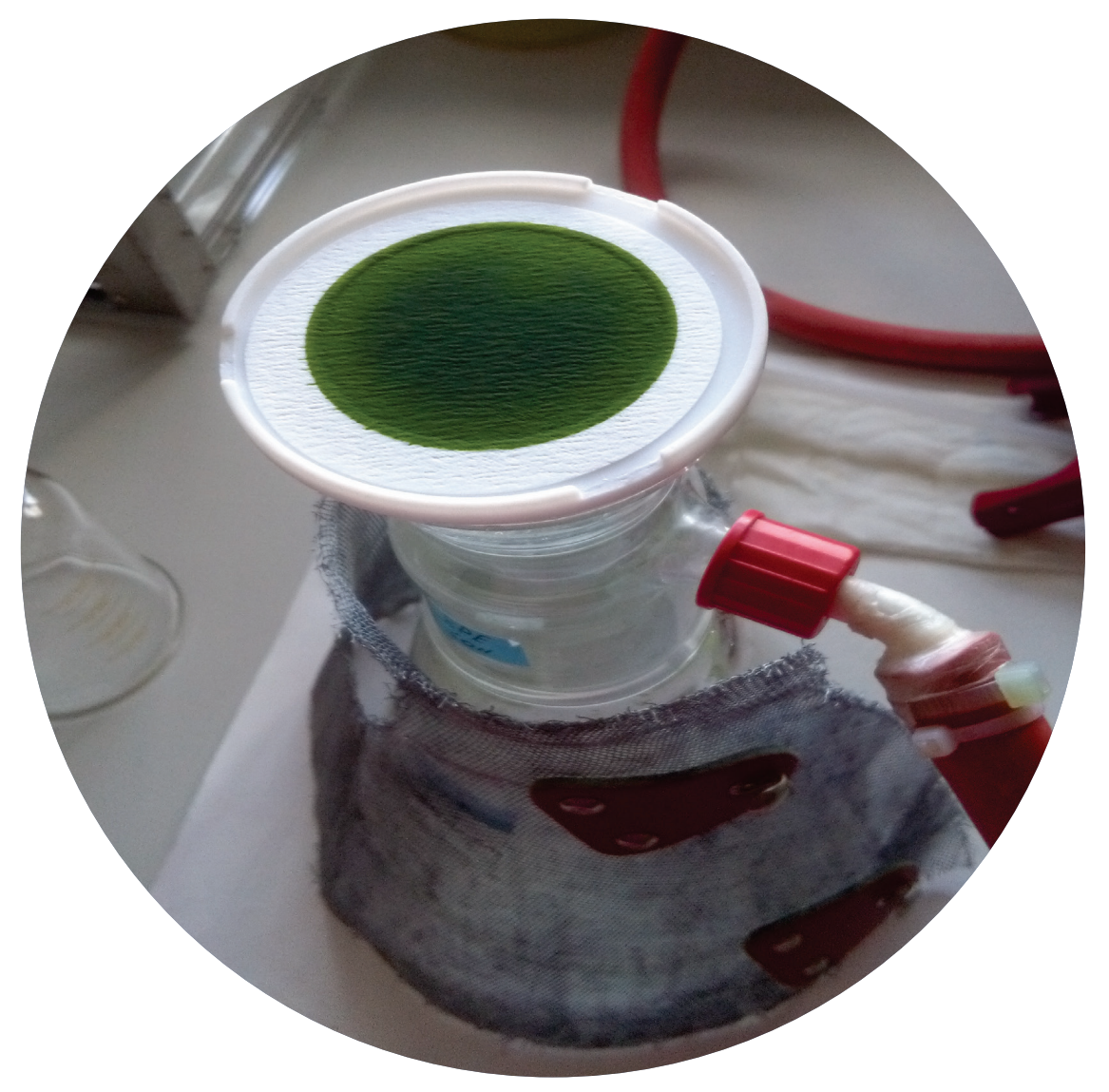




\section{Chapter}

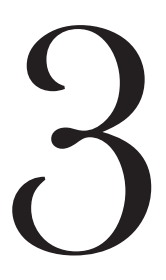

\section{The diurnal transcriptional landscape of the microalga Tetradesmus obliquus}


Tetradesmus obliquus is a promising oleaginous microalga for the production of biofuels. Its physiological responses have been studied under light/dark (LD) cycles, showing that starch is used as temporary energy storage. However, little is known about the scheduling of cellular processes. In this work, we looked into the diurnal changes of the transcriptome profile of T. obliquus adapted to 16:8 h LD cycles grown under turbidostat culture conditions. First, its genome was annotated resulting in 19,795 genes, which encode 21,493 transcripts, and further translate into 19,723 protein sequences. When combining the annotated genome with gene expression information, we identified 4,686 genes that had a significant change of expression level during the diurnal LD cycle. Global principal component analysis of $T$. obliquus wild-type shows that samples are arranged in a clocklike pattern. This indicates that gene expression is impacted mainly by two effects: light availability and time of the day. Six time phases were identified based on the expression similarities between individual samples. Using a clustering of genes based on their expression similarity, again 6 clusters peaking at different times of the day were observed. The time phases correlated with peaks of expression from the gene clusters. Functional analysis of the genes in each cluster revealed a clear succession of cellular events. Additionally, these transcriptional cellular phenotypes link to biochemical measurements from this alga. Furthermore, we also studied the impact of the lack of starch on the timing of gene expression using the T. obliquus starchless mutant $s / m 1$. When analyzing the data from $s / m 1$, significant changes in the gene temporal profiles were identified. Most of the detected differences between strains can be explained by a time shift or by changes in amplitude of the peaks, with only a few genes displaying a pattern clearly distinct from their wild-type expression profile. Overall, two main trends were observed for the $\operatorname{sim1}$ : earlier expression of processes shortly after the light went on followed by a delay of expression of the processes shortly before the dark period. 


\subsection{Introduction}

Microalgae are a promising source of compounds of interest (lipids, proteins, and pigments) for production of food, feed, chemicals, and fuels (Wijffels et al., 2010; Wijffels and Barbosa, 2010; Guarnieri and Pienkos, 2015). Large scale microalgal production will be primarily done outdoors under natural diurnal light/dark (LD) cycles (Norsker et al., 2011; Blanken et al., 2013). Diurnal cycles are ubiquitous and photosynthetic organisms synchronize their metabolic activities to anticipate light changes in the environment (McClung, 2006; Farré, 2012; de Winter et al., 2013; LeónSaiki et al., 2017). Synchronization in photosynthetic organisms involves the regulation of photosynthesis to maximize carbon fixation and use of light during the day, and to schedule light sensitive processes (such as DNA synthesis and cell division) at night (Nikaido and Johnson, 2000; Suzuki and Johnson, 2001; de Winter et al., 2013).

Tetradesmus obliquus is a microalga recognized as an industrially relevant strain for food and fuel production (Breuer et al., 2012; Remmers et al., 2017). T. obliquus can reach a maximum triacylglycerides (TAG) content of $0.45 \mathrm{~g} \cdot \mathrm{g}_{D W^{-1}}$ and a maximum TAG yield on light of $0.14 \mathrm{~g} \cdot \mathrm{mol}_{\mathrm{ph}}{ }^{-1}$ under batch nitrogen starvation and continuous light conditions (Breuer et al., 2014). For further improvement of TAG yield on light, de Jaeger et al. (2014) developed the starchless mutant s/m1, which cannot synthesize starch due to a mutation in the small subunit of ADP-glucose pyrophosphorylase, the committed step of starch biosynthesis (de Jaeger, 2015). Under the culture conditions of continuous light and batch nitrogen starvation, the s/m1 mutant showed a higher maximum TAG yield on light $\left(0.217 \mathrm{~g} \cdot \mathrm{mol}_{\mathrm{ph}}{ }^{-1}\right)$ and maximum TAG content $(0.57$ $\left.\mathrm{g} \cdot \mathrm{g}_{\mathrm{DW}}{ }^{-1}\right)$ compared to the wild-type without a decrease in photosynthetic efficiency (Breuer et al., 2014).

The physiological behavior of T. obliquus wild-type and starchless mutant ( $s / m 1)$ has also been studied under 16:8 h LD cycles in turbidostat controlled systems (León-Saiki et al., 2017; León-Saiki et al., 2018). Under this light regime and nitrogen replete conditions, $T$. obliquus showed synchronization and diurnal patterns of its metabolism, which amongst others, indicated that starch was used as a temporary energy storage. This is, starch was accumulated during the light period and was used during the dark period. Cell division was also synchronized and occurred during the night. Notably the energy conversion efficiency and the yield of biomass on light were higher under LD 
cycles than under continuous light. The starchless mutant $\operatorname{s} / m 1$ showed a lower energy conversion efficiency (11 to $24 \%$ lower) and biomass yield on light (13 to 39\% lower) compared to the wild-type for different photoperiods (León-Saiki et al., 2018). Furthermore, for the $\operatorname{s} / m 1$ mutant, cell division still occurred mainly during the night, but at a slower rate and no diurnal oscillations in any of the other measured compounds were found (León-Saiki et al., 2018). Unlike for the wild-type, the diurnal LD cycles did not provide an advantage for the $\operatorname{s} / m 1$ mutant compared to continuous light, as the biomass yield on light as well as the energy conversion efficiency was similar under both conditions (León-Saiki et al., 2017). This indicates that starch may play an important role in harvesting additional light energy during LD cycles.

While the biochemical analysis allowed us to draw some useful conclusions, we still lack information on how its different cellular processes are regulated during the diurnal cycle and how the synthesis and use of starch is connected to them. Furthermore, while the inability to make starch had an impact on energy conversion efficiency, it is not known how timing and regulation of the different processes are affected in s/m1. LD cycles and the subsequent synchronization of the microalgal population makes it of paramount importance to unravel the timing of cellular and subcellular events. Understanding the timing at which metabolic changes take place is essential to understand the phenotype exhibited by the wild-type and mutant strains, and to optimally design experiments characterizing mutant strains.

Time resolved transcriptome analysis can give useful insights into the timing, regulation, and order of the different physiological processes in the cell. Furthermore, it allows the association of the cellular phenotype (gene expression) with the biochemical measurements. Algal diurnal transcriptional regulation is not well known. To our knowledge, only a few reports on diurnal oscillations under LD cycles in microalgae have been published (Fujiwara et al., 2009; Chauton et al., 2013; Poliner et al., 2015; Zones et al., 2015; Suzuki et al., 2016). Fujiwara et al. (2009) studied transcriptional changes in the eukaryotic red alga Cyanidioschyzon merolae grown under 12:12 h LD cycle in intervals of $2 \mathrm{~h}$. Chauton et al. (2013) studied the diurnal transcriptional changes in the diatom Phaedactylum tricornutum under 16:8 h LD cycles. The authors studied changes in biochemical composition (carbohydrates and lipids) in 8 different unequally distributed time points during a period of 26.5 hours (5 points during the light period and 3 during the dark period). Poliner et al. (2015) 
reported on Nannochloropsis oceanica and Zones et al. (2015) on Chlamydomonas reinhardtii under a 12:12 h LD cycle. For N. oceanica the authors studied growth, changes in biomass composition (lipids and glucose) and in gene expression in interval of $3 \mathrm{~h}$. While for $C$. reinhardtii a more detailed studied was done with intervals of $1 \mathrm{~h}$ during most of the cycle and every $30 \mathrm{~min}$ for some time points distributed over the $24 \mathrm{~h}$ cycle. Our study, however, is the first one to look into the transcriptional changes in a diurnal cycle of an oleaginous green alga with a high sampling frequency (intervals of $1 \mathrm{~h}$ ). Additionally, this is the first study reporting on time resolved gene expression in a starchless mutant, which could give insight into the role of starch in microalgae.

To fully characterize the changes induced by LD cycles and the role of starch metabolism, we analyzed and compared the transcriptional landscape of T. obliquus wild-type and s/m1 under diurnal 16:8 h LD cycles. For this, we cultivated both strains in continuous turbidostat controlled photobioreactors. Samples were taken after steady-state was reached in which the algae grew synchronized to the LD cycle. For the wild-type, sampling was done in intervals of $1 \mathrm{~h}$, for high resolution, while for $\operatorname{sim} 1$ sampling was done at $3 \mathrm{~h}$ intervals.

\subsection{Materials and Methods}

\subsubsection{Experimental set-up and sampling}

Wild-type Tetradesmus obliquus UTEX 393 (formerly known as Acutodesmus obliquus and Scenedesmus obliquus (Krienitz and Bock, 2012)) was obtained from the Culture Collection of Algae, University of Texas. The starchless mutant of T. obliquus (s/m1) was generated as described by de Jaeger et al. (2014). T. obliquus was continuously cultivated in a sterile flat panel airlift-loop reactor with a $1.7 \mathrm{~L}$ working volume (Labfors 5 Lux, Infors HT, Switzerland). Pre-culture conditions and reactor set-up $\left(27.5^{\circ} \mathrm{C}, \mathrm{pH}\right.$ 7.0 and gas flow rate of $1 \mathrm{~L} \cdot \mathrm{min}^{-1}$ air enriched with $2 \% \mathrm{CO}_{2}$ ) were controlled as described by León-Saiki et al. (2017). Light was provided in a 16:8 h light/dark (LD) block at an incident photon flux density of $500 \mu \mathrm{mol} \cdot \mathrm{m}^{-2} \cdot \mathrm{s}^{-1}$. Cultivations were turbidostat controlled, where fresh medium was fed to the cultures when the light intensity at the rear of the reactor dropped below the setpoint $\left(10 \mu \mathrm{mol} \cdot \mathrm{m}^{-2} \cdot \mathrm{s}^{-1}\right)$. Feeding of medium was stopped during the dark period. After steady state was reached, $8 \mathrm{~mL}$ samples

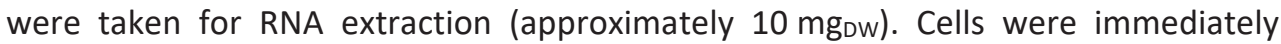


collected by centrifugation ( $4255 \times \mathrm{g}, 0^{\circ} \mathrm{C}$ for $5 \mathrm{~min}$ ), supernatant was discarded and pellets were frozen in liquid nitrogen and stored at $-80^{\circ} \mathrm{C}$ until further extraction. Samples were taken in intervals of one hour for the wild-type or every three hours for the $\operatorname{sim1}$. Due to restrictions on working hours of the laboratory, the samples were collected in two successive parts to allow sampling the dark period during the day. After collecting samples of the first half of the cycle, light settings were shifted and the culture was then allowed to reach steady state again before collecting samples for the second half of the cycle. The first and the last samples of each measured half cycle are overlapping samples to verify that the same steady state was reached. Therefore, four RNA samples are present at these overlapping time points. Overall 72 samples were taken for RNA extraction.

\subsubsection{RNA isolation and quality control}

RNA extraction was performed using the Maxwell ${ }^{\circledR} 16$ LEV simplyRNA Tissue kit (Promega). Frozen algae pellet $(\approx 200 \mu \mathrm{L})$ were submerged in $400 \mu \mathrm{L}$ of homogenizing buffer supplemented with $8 \mu \mathrm{L}$ 1-thioglycerol in a $2 \mathrm{~mL}$ Lysing matrix $C$ tube (MP), prefilled with a mix of glass beads. Samples were disrupted using a FastPrep-24 instrument (MP). After disruption, all liquid was transferred to a LEV RNA Cartridge. $300 \mu \mathrm{L}$ lysis buffer were added and the rest of the extraction was performed using a Maxwell MDx AS3000 machine (Promega) following manufacturer's instructions. RNA integrity and quantity were assessed with an Experion system (Bio-Rad), and only high quality samples (RIN value $\geq 7$ ) were selected. Total RNA was sent for whole transcriptome sequencing to Novogene Bioinformatics Technology Co. Ltd (Hong Kong, China).

\subsubsection{Genome structural annotation}

Using the available genome sequence of T. obliquus (Carreres et al., 2017), we performed an RNA-Seq-based genome annotation using BRAKER1 (Hoff et al., 2016). RNA reads from 38 samples of both the wild-type and the $s / m 1$, and one additional sample from each strain but under nitrogen limited condition (not further discussed here) were given as additional BRAKER1 input. The annotation information was processed using our semantic framework pipeline (Koehorst et al., 2018) and the information was stored according to our integrated ontology (Dam et al., 2017) which 
respects the FAIR Data principles (Wilkinson et al., 2016). The annotation framework and the ontology were extended with the necessary tools (Trapnell et al., 2010; Dobin et al., 2013; Hoff et al., 2016) and ontology terms for the purpose of this analysis.

\subsubsection{Genome functional annotation and pathway mapping}

Proteins were annotated to GO terms using InterProScan5 and Argot2, with default parameters (Falda et al., 2012; Jones et al., 2014). Proteins were annotated to enzyme commission (EC) number using EnzDP (Nguyen et al., 2015). Results with a complete EC number and a likelihood-score of at least 0.2 were used for subsequent analysis. To choose a threshold for the likelihood-score that results in a good trade-off between true positive and false positives, we visually compared the completeness of KEGG metabolic maps while avoiding dispersion of each reaction into different expression clusters.

\subsubsection{RNA-seq normalization and expression calculation}

Using all the transcripts found from the genome annotation, we aligned the reads from each sample and calculated the FPKM values using Cufflinks (Trapnell et al., 2010). As a pre-filter to remove unexpressed genes and false positives from gene annotation, we selected the transcripts with a coverage of at least 1 and a FPKM of at least 0.1 in at least 10 of the 113 analyzed samples. These samples include the 72 of this study, and the rest were taken under LD cycles and under nitrogen limited conditions, but are not discussed in this manuscript. Additionally, we selected the transcripts having an expression of at least 10 samples with a value higher or equal to the 0.15 quantile. Following the advice of maSigPro (Nueda et al., 2014), the FPKM of all samples were normalized using the scaling normalization method TMM (Robinson and Oshlack, 2010) using the R functions from edgeR package "calcNormFactors" (Robinson et al., 2010).

\subsubsection{Gene clustering}

To identify genes with significant expression profile changes over time, maSigPro was used with default parameters with a few exceptions (Äijö et al., 2014; Nueda et al., 2014). The regression model was set to a maximum of 23 degrees, the parameter 
"counts" was set to true, "nvar.correction" was also set to true, the "step method" set as backward, and R-squared was set to 0.7 . The R-squared was chosen based on the relevance and the balance in the number of enriched pathways identified in each cluster (see below). To better estimate the number of clusters to group the gene expression, we evaluated combinations of significance level ( $Q: 0.005$ to 0.05 ) and $R$ squared $(0.85$ to 0.5$)$. We decided to keep the standard-strict values $(Q=0.05, R$ squared=0.7), which would also give a good balance in pathway enrichment and number of genes in each group.

Hierarchical clustering with agglomerative linkage was performed using the $\mathrm{R}$ stats package (hclust function). Number of clusters from 3 to 25 were evaluated using the $R$ function "cluster.stats" from package "fpc" (Hennig, 2018). For each set of clusters, the indexes for average silhouette widths, normalized gamma, two Dunn indexes, average within average between ratio, and Calinski and Harabasz index were computed for each cluster separations and each set of clusters were compared one on one with the Rand index.

\subsubsection{Enrichment analyses}

Enrichment analyses were performed using the hypergeometric function to model the probability density using the "phyper" function from the R package stats (R Core Team, 2017). Two types of analysis were performed: pathway and GO term enrichment. Pathway enrichment required associating annotated Enzyme Commission (EC) numbers to metabolic maps. We used the online available resource from KEGG pathway maps (Kanehisa and Goto, 2000; Kanehisa et al., 2016). The KEGG pathways fitting the following requirements were kept for further analysis: at least $25 \%$ of coverage for the annotated EC numbers in the pathway map, at least 3 EC numbers annotated, $60 \%$ coverage if 3 to 6 EC numbers annotated, and 50\% coverage if 6 to 10 EC numbers annotated. For the hypergeometric test we considered the universe size, $N$, to be the total number of EC numbers in all pathways in the genome, $m$ is the number of successes in the universe and is defined as the number of EC numbers in the corresponding pathway in the genome, $k$ and $x$ are the sample size and the number of successes in the sample (or considered gene subset) respectively. Similarly, for the GO enrichment, $N$ is the total number of genes annotated to any GO terms in the genome, $m$ is defined by the number of genes annotated to the considered GO term in 
the genome, and $k$ and $x$ refer to the considered subset of genes. Multiple test correction for the GO enrichment was performed using the Benjamini-Hochberg procedure. To handle the GO information such as ontology, ancestor, and offspring, we used "GO.db" database from bioconductor (Carlson et al., 2007). Additionally, to reduce the number of $\mathrm{GO}$ terms and conserve the most specific, only the terms not having any offspring in the selection were retained. For both enrichment analysis, results with a p-value lower or equal to 0.05 were considered as significant. We additionally provide the pathway coverage, and the $x$ and $m$ values to better understand the reason of each significant enrichment.

\subsection{Results}

\subsubsection{Genome annotation}

The genome of $T$. obliquus UTEX 393, submitted in 2016, is about 100 million base pairs separated in 1,368 scaffold sequences (Carreres et al., 2017). The BRAKER1 annotation revealed 19,795 genes, which transcribed 21,493 coding sequences, that translated into 19,723 unique protein sequences. The difference in the numbers of coding sequences and unique protein sequences is the result of transcripts with different sequences but coding for the same protein sequence.

To analyze the changes in transcription over a diurnal cycle of $T$. obliquus wild-type and slm1 strains, we sequenced RNA from biological duplicate runs. T. obliquus wild-type was sampled every hour in duplicate resulting in 52 samples. $\operatorname{sim} 1$ was sampled in intervals of three hours resulting in 20 samples. For both strains, time points $0 \mathrm{~h}$ and $13 \mathrm{~h}$ were overlapping samples and therefore sequenced in quadruplicate (see materials and methods for more details). Not all the transcripts were found to be expressed and filtering was performed to identify genes with very low expression that were not further considered. From the 19,723 proteins, 16,810 proteins remained after filtering for subsequent analysis. GO term annotation yielded 13,687 proteins that matched to 2,394 unique GO terms. EC annotation yielded 3,559 proteins that matched to 1,315 EC numbers. 


\subsubsection{Light/dark cycles induce systemic transcriptional changes following a circular pattern}

4,686 genes were found to have a significant change of expression during the diurnal cycle. A principal component (PC) analysis for these selected genes is shown in Figure 3.1A. The PC plot provides a global overview of changes in gene expression over time. The time point samples form an ordered and circular pattern over the two first PCs. These two first PCs together explain $87 \%$ of the variation in expression. To demonstrate that this is not a consequence of gene selection, we rendered another PC analysis considering the whole set of expressed genes (data not shown). Within this set, the overall pattern is noisier, but the two first components still explain $78 \%$ of the variation in expression. The main changes in gene expression occur along PC1 from $1 \mathrm{~h}$ to about $5 \mathrm{~h}$ and back again from $8 \mathrm{~h}$ to about $13 \mathrm{~h}$. The second important change occurs along PC2 from $6 \mathrm{~h}$ to $9 \mathrm{~h}$ and back again from about $22 \mathrm{~h}$ to $0 \mathrm{~h}$. During the period from $14 \mathrm{~h}$ to $22 \mathrm{~h}$ there is very little change in gene expression. Overall, genes associated to light harvesting complex (LHC) were found to be the main contributors of PC1. The two highest contributing genes to PC2 are glyceraldehyde-3-phosphate dehydrogenase (GA3PDH) and glucose-6-phosphate isomerase (GPI). LHC genes also contributed strongly to PC2.

The heatmap representation in Figure 3.1B offers a complementary view of these multi-dimensional data. It allows the evaluation of differences and similarities in expression between the time points. Two samples corresponding to time points $13 \mathrm{~h}$ and $15 \mathrm{~h}$ are separated in the dendrogram from the neighboring samples and from the biological duplicate samples. This separation results from the very low correlation in gene expression of these time points with the other time points that are several hours apart, whereas for the biological replicates and the surrounding hours the correlation is high. Three main groups of time points can be identified in the dendrograms in Figure 3.1B, which we will refer to as time phases I, II, and III. Additionally, each phase can be subdivided into two sub-phases, $a$ and $b$, which represent subtler expression changes. The occurrence of the phases over the 16:8 h LD cycle is shown in Figure 3.1E for both strains. The phases were named according to their order of appearance from $\mathrm{O} \mathrm{h}$ ("sunrise") to $23 \mathrm{~h}$. These time phases are also apparent in the PC plots. Similarly, time phases were also associated to the $\operatorname{s} / m 1$ strain based on the PC analysis plots. 

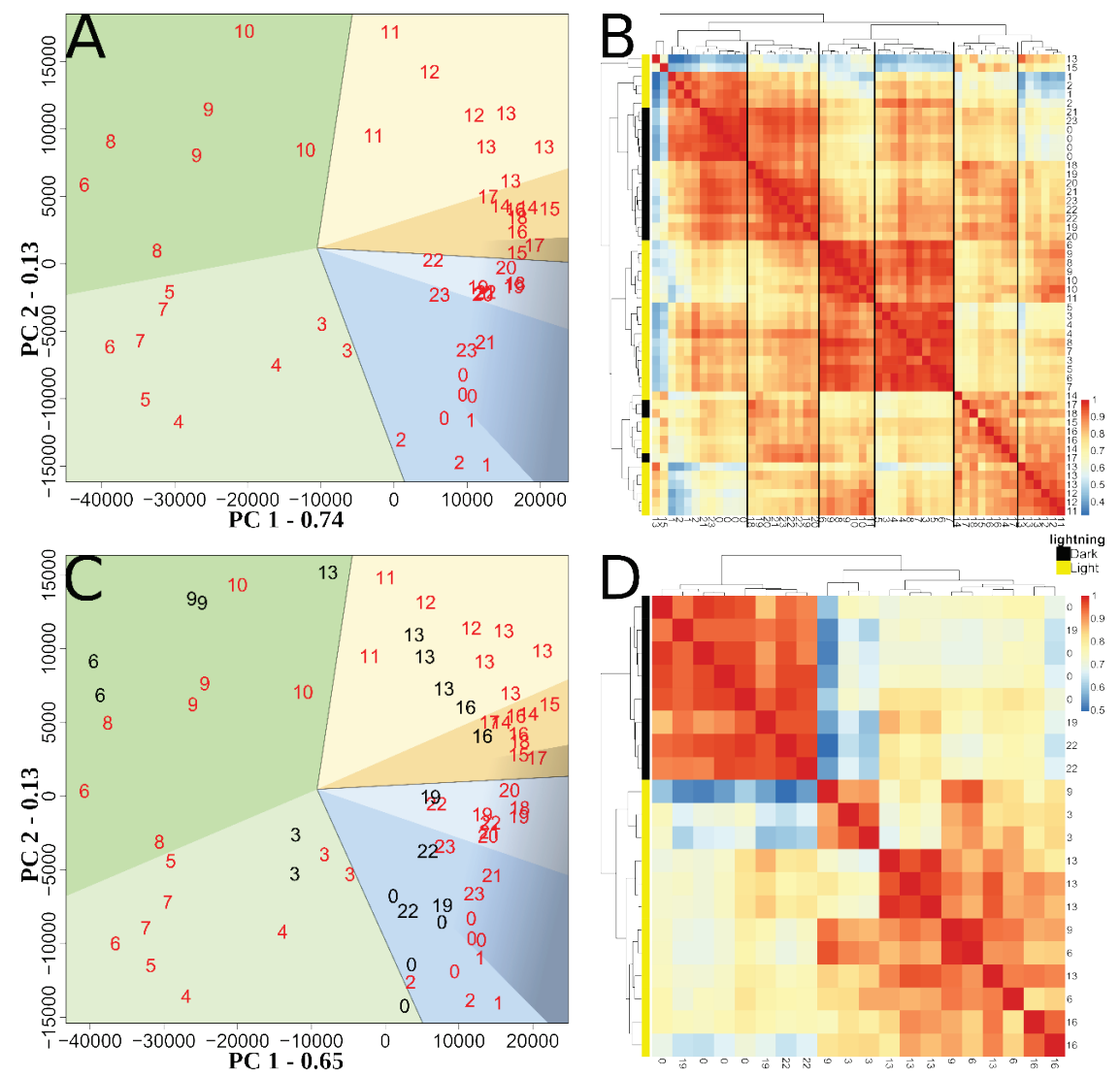

\begin{tabular}{|c|c|c|c|c|c|c|c|c|c|c|c|c|c|c|c|c|c|c|c|c|c|c|c|c|c|}
\hline \multicolumn{2}{|c|}{$E_{\text {Time }}(h)$} & 0 & 1 & 2 & 3 & 4 & 5 & 6 & 7 & 8 & 9 & 10 & 11 & 12 & 13 & 14 & 15 & 16 & 17 & 18 & 19 & 20 & 21 & 22 & 23 \\
\hline \multicolumn{2}{|c|}{ Light } & \multicolumn{17}{|c|}{ ON } & \multicolumn{7}{|c|}{ OFF } \\
\hline ฆี & WT & \multicolumn{3}{|c|}{ Ia } & \multicolumn{5}{|c|}{ IIa } & & IIb & & \multicolumn{3}{|c|}{ IIIa } & \multicolumn{4}{|c|}{ IIIb } & \multicolumn{5}{|c|}{ Ib } & Ia \\
\hline$\overline{1}$ & MT & Ia & & & IIa & & & IIb & & & IIb & & & & IIIa & & & IIIIb & & & $\mathbf{I b}$ & & & Ia & \\
\hline
\end{tabular}

Figure 3.1. Principal Component Analysis (PCA) of gene expression data for Tetradesmus obliquus wild-type (A) and both wild-type and starchless mutant s/m1 (C) during 16:8h light/dark cycle. Heatmaps showing similarity of gene expression data among samples for the wild-type (B) and $s / m 1$ (D) strain. Overview of the time phases (E). Numbers in (A) represent the time points of the samples, with red for the wild-type and black for the $\operatorname{sim} 1$ samples. Background colors in both PCA plots refer to the colors given to the six time sub-phases described in (E), and the dark shade refers to the approximate dark period. The dark lines merging at the center of both PCA plots separate the three time phases for the wild-type. Dark vertical lines across the heatmap in (B) indicate different time phases. The $x$ axis indicates hours after 'sunrise'. 
However, for $\operatorname{s} / m 1$ we noticed a change in phase timing that represents a significant change of expression occurring one to two hours before each dark-light shift.

\subsubsection{Gene expression clusters and time profiles}

\section{Cluster profiles}

We clustered the 4,686 genes that showed significant changes over time in different numbers of clusters ranging from 3 to 25 . Next we evaluated the cluster separation using 7 well established indexes to assess similarities within clusters and differences between clusters (data not shown). Based on this we opt for separating the genes in 6 clusters.

The resulting six cluster profiles were summarized using the median points and are depicted in Figure 3.2. All these profiles are different, but there are certain similarities between clusters 1, 2 and 4, and between clusters 3 and 5 . Clusters 2 and 1 both show two peaks of expression throughout the $24 \mathrm{~h}$ cycle, with a peak at the end of the light period and one at the beginning of the dark period. However, cluster 2 shows a time shift with the two peaks appearing $2 \mathrm{~h}$ and $1 \mathrm{~h}$ earlier, respectively. Cluster 4 also shows two peaks, one at the very end of the day and a relatively low one in the night. Expression of cluster 3 rises continuously from $5 \mathrm{~h}$ until the end of the night, and drops sharply when the light goes on (time $0 \mathrm{~h}$ ). Cluster 5 is different from cluster 3 because the continuous rise is interrupted by a small peak and dip at $15 \mathrm{~h}$ and $17 \mathrm{~h}$, respectively. Furthermore, the peak and sharp drop right after light goes on, as well as the start of the continuous increase, occur 1 hour later. As for cluster 6 , the profile displays an early day expression peaking at $3 \mathrm{~h}$ and decreasing fast until $12 \mathrm{~h}$, followed by another slow increase until beginning of the dark period where it stabilizes.

The temporal profile of each cluster peaks at different moments of the diurnal cycle. Cluster 5 peaks the earliest, $1 \mathrm{~h}$ after light goes on. Cluster 6 peaks at $3 \mathrm{~h}$, cluster 2 peaks around $10 \mathrm{~h}$ and shows a smaller secondary peak at $17 \mathrm{~h}$. Cluster 1 , with a similar profile, shows the first peak at $13 \mathrm{~h}$ and the second at $18 \mathrm{~h}$. Cluster 4 has a rather gradual increase of expression and peaks around $14 \mathrm{~h}$ followed by a smaller peak at $20 \mathrm{~h}$. Finally, cluster 3 has very gradual expression changes throughout the day, and shows a peak at $23 \mathrm{~h}$. 


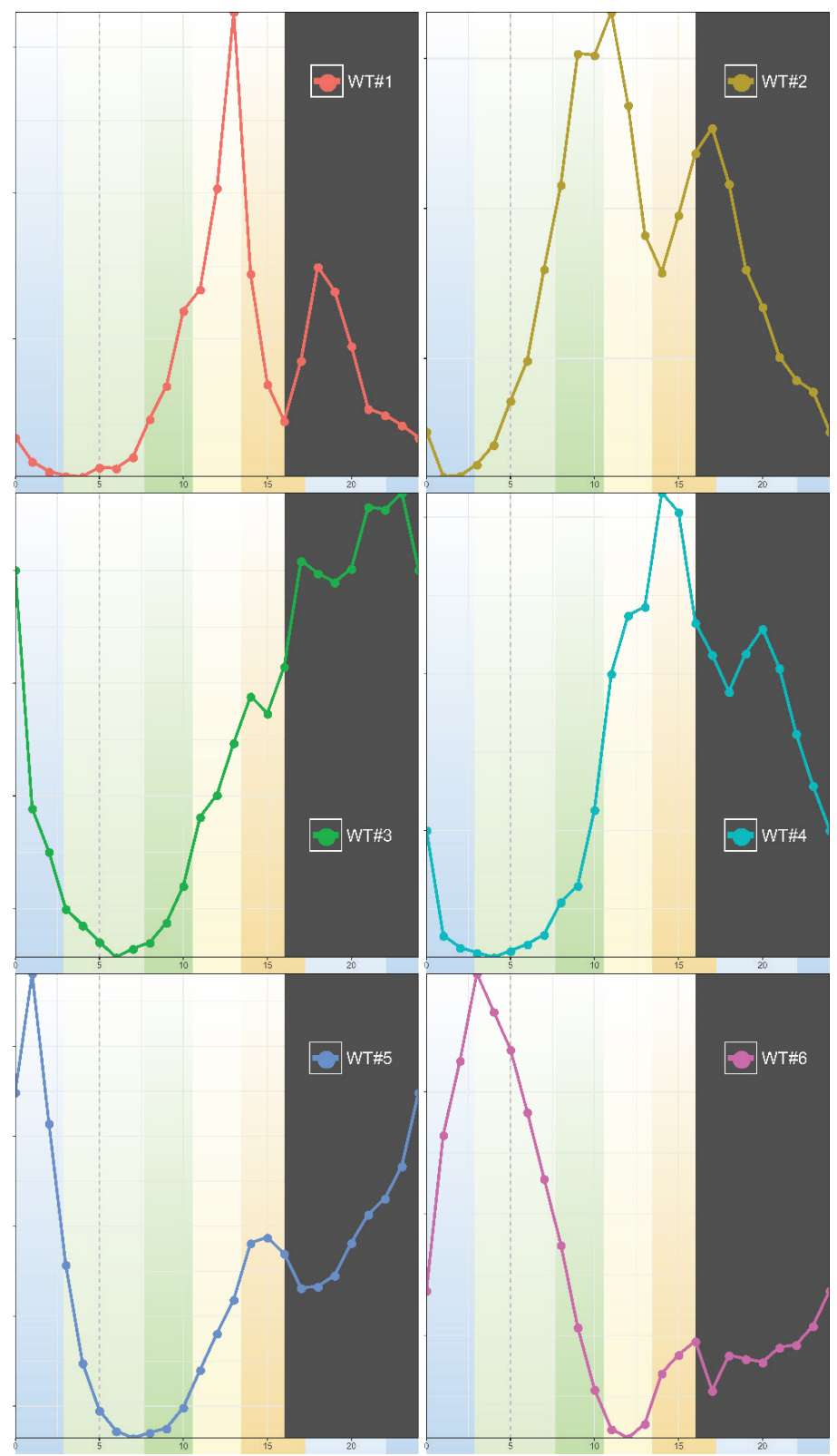

Figure 3.2. Expression profile of the 6 gene clusters for Tetradesmus obliquus under 16:8 $\mathrm{h}$ light/dark cycles. The plots show the median profile of the genes in all clusters. The name of the cluster is specified in the legend. The colors in the background of the time points correspond to the colors given to the time phases in Figure 3.1. The vertical gray dashed line represents the time point of maximum dilution rate taken from León-Saiki et al. (2017). Shaded area indicates the dark period. 


\section{Cluster functional analyses}

To understand the functions of the genes in the clusters, we performed two sets of complementary enrichment analyses. Metabolic pathway enrichment analysis provides direct information on gene metabolic functions. The functional annotation identified genes associated to EC numbers. Mapping these EC numbers to KEGG pathway maps revealed 67 pathways for which enough genes can be associated to the corresponding reactions to warrant further analysis, as detailed in Materials and methods. GO enrichment analyses were performed by considering the three ontologies: biological process (BP), molecular function (MF) and cellular component (CC). These analyses provide a wider overview not restricted to metabolism. The enrichment results are summarized in Table 3.1.

Table 3.1. Pathway enrichment and GO enrichment analysis for the 6 gene clusters found for Tetradesmus obliquus wilt-type under 16:8 h light/dark cycles. The three GO ontologies have been used (biological process (BP), molecular function (MF) and cellular component (CC))

\begin{tabular}{|c|c|c|}
\hline Cluster & Pathways & GO terms \\
\hline 1 & $\begin{array}{l}\text { Galactose metabolism } \\
\text { Starch and sucrose metabolism } \\
\text { Other glycan degradation } \\
\text { Amino sugar and nucleotide sugar } \\
\quad \text { metabolism } \\
\text { Metabolism of xenobiotics by } \\
\text { cytochrome P450 } \\
\text { Drug metabolism - cytochrome P450 }\end{array}$ & $\begin{array}{l}\text { BP: } \\
\text { carbohydrate metabolic process } \\
\text { MF: } \\
\text { hydrolase activity, hydrolyzing O-glycosyl } \\
\quad \text { compounds } \\
\text { microtubule binding } \\
\text { polysaccharide binding }\end{array}$ \\
\hline 2 & $\begin{array}{l}\text { Lysine degradation } \\
\text { Phenylalanine metabolism } \\
\text { Glutathione metabolism } \\
\text { N-Glycan biosynthesis } \\
\text { Various types of N-glycan } \\
\text { biosynthesis } \\
\text { Propanoate metabolism }\end{array}$ & $\begin{array}{l}\text { BP: } \\
\text { DNA replication } \\
\text { DNA repair } \\
\text { DNA recombination } \\
\text { protein complex assembly } \\
\text { vesicle-mediated transport } \\
\text { cell cycle process } \\
\text { regulation of cellular metabolic process } \\
\text { proteasome-mediated ubiquitin- } \\
\quad \text { dependent protein catabolic process } \\
\text { organelle fission } \\
\text { chromosome organization } \\
\text { regulation of primary metabolic process } \\
\text { carbohydrate derivative biosynthetic } \\
\quad \text { process } \\
\text { single-organism organelle organization }\end{array}$ \\
\hline
\end{tabular}


microtubule cytoskeleton

protein complex

cytoskeletal part

MF:

DNA binding

DNA-directed DNA polymerase activity

ATP binding

microtubule binding

transcription factor binding

four-way junction helicase activity

ATPase activity, coupled

\begin{tabular}{|c|c|c|}
\hline 3 & $\begin{array}{l}\text { Fatty acid degradation } \\
\text { Pyrimidine metabolism } \\
\text { Butanoate metabolism } \\
\text { Folate biosynthesis }\end{array}$ & \\
\hline 4 & $\begin{array}{l}\text { Glycolysis / Gluconeogenesis } \\
\text { Glutathione metabolism } \\
\text { Starch and sucrose metabolism } \\
\text { Sphingolipid metabolism } \\
\text { Pyruvate metabolism } \\
\text { Glyoxylate and dicarboxylate } \\
\quad \text { metabolism } \\
\text { Thiamine metabolism } \\
\text { Pantothenate and CoA biosynthesis } \\
\text { Nitrogen metabolism } \\
\text { Metabolism of xenobiotics by } \\
\quad \text { cytochrome P450 } \\
\text { Drug metabolism - cytochrome P450 }\end{array}$ & $\begin{array}{l}\text { BP: } \\
\text { carbohydrate metabolic process } \\
\text { oxidation-reduction process } \\
\frac{\text { MF: }}{\text { oxidoreductase activity, acting on } \mathrm{CH}-\mathrm{OH}} \\
\quad \text { group of donor }\end{array}$ \\
\hline 5 & $\begin{array}{l}\text { Ubiquinone and other terpenoid- } \\
\text { quinone biosynthesis } \\
\text { Purine metabolism } \\
\text { Glycine, serine and threonine } \\
\text { metabolism } \\
\text { Cysteine and methionine } \\
\text { metabolism } \\
\text { Valine, leucine and isoleucine } \\
\text { biosynthesis } \\
\text { Lysine biosynthesis } \\
\text { Arginine and proline metabolism } \\
\text { Riboflavin metabolism } \\
\text { Aminoacyl-tRNA biosynthesis }\end{array}$ & $\begin{array}{l}\text { BP: } \\
\text { RNA methylation } \\
\text { tRNA modification } \\
\text { translation } \\
\text { nucleobase-containing small molecule } \\
\quad \text { metabolic process } \\
\text { alpha-amino acid biosynthetic process } \\
\text { CC: } \\
\text { ribosome } \\
\text { MF: } \\
\text { RNA binding } \\
\text { Structural constituent of ribosome } \\
\text { GTPase activity } \\
\text { GTP binding } \\
\text { S-adenosylmethionine-dependent } \\
\quad \text { methyltransferase activity } \\
\text { ligase activity, forming carbon-nitrogen } \\
\quad \text { bonds }\end{array}$ \\
\hline 6 & $\begin{array}{l}\text { Glycine, serine and threonine } \\
\text { metabolism } \\
\text { Phenylalanine, tyrosine and } \\
\text { tryptophan biosynthesis }\end{array}$ & $\begin{array}{l}\text { BP: } \\
\text { tRNA aminoacylation for protein } \\
\quad \text { translation } \\
\text { steroid biosynthetic process } \\
\text { coenzyme metabolic process }\end{array}$ \\
\hline
\end{tabular}


Carbon fixation in photosynthetic organisms

Porphyrin and chlorophyll metabolism

Terpenoid backbone biosynthesis

Carotenoid biosynthesis

Aminoacyl-tRNA biosynthesis

\author{
porphyrin-containing compound \\ biosynthetic process \\ photosynthesis \\ pigment biosynthetic process \\ oxidation-reduction process \\ CC: \\ Photosystem
}

Cluster 5 shows enrichment in pathways related to amino acids, and riboflavin metabolism. GO terms enrichment is in agreement and contains terms suchs as tRNA modification, aminoacyl-tRNA biosynthesis, ribosome, alpha-amino acid biosynthetic process and translation. All these indicate a strong increase in amino acids and protein synthesis.

Cluster 6, also shows enrichment in genes related to transcription, amino acid and protein synthesis, but to a lower extent than cluster 5 . The carbon fixation pathway is found enriched in cluster 6, which goes together with enrichment in the pigments and in starch synthesis. The enrichment in synthesis of pigments, which include carotenoids and chlorophylls, agrees with enrichment of the synthesis of their precursor (phytyldiphosphate) in the terpenoids backbone synthesis pathway. The pigments are needed for building the photosystems and starting the carbon fixation. The GO terms in 
biological process are in agreement with the pathway enrichments. The GO terms in molecular function provide extra information on the nature of chemical reactions performed by these genes. We also found that this cluster contains genes associated to the LHC that were found to strongly contribute to PC1 and PC2.

Cluster 2 is also enriched with amino acids pathways: lysine degradation and phenylalanine metabolism. Additionally, the enrichment in N-Glycan biosynthesis and in various types of $\mathrm{N}$-glycan biosynthesis, together with the enriched GO terms "protein complex assembly" and "vesicle-mediated transport" indicates important protein maturation processes leading to complex proteins and some transport of proteins to membranes. In this cluster, the GO term enrichment displays clear terms related to cell cycle progression, such as "DNA replication", "organelle fission", "chromosome organization", "microtubule", "DNA polymerase activity". This is a nice example of valuable information that is obtained from the GO enrichment and that cannot be obtained from the pathway enrichment.

Cluster 1 is mostly enriched in pathways associated to different kind of carbohydrates metabolism. The first pathways enriched are galactose metabolism, starch and sucrose metabolism, other glycan degradation, and amino sugar and nucleotide sugar metabolism. With the GO terms displaying "polysaccharide binding", "hydrolase activity", and "hydrolyzing O-glycosyl", we observed the same trend. The GO term for "microtubule binding" is the only one of this kind in this cluster, but it comes right after cluster 2 where a lot of cytoskeletal and microtubule terms were found enriched. Finally, there is enrichment in Cytochrome P450 which hints on repair mechanisms potentially related to photo-damage.

Cluster 4 shows enrichment in a very diverse set of metabolic pathways, covering glycolysis, pyruvate metabolism, glutathione metabolism, co-enzyme-A, starch and other carbohydrates polymers. More importantly, the cluster is also enriched in genes related to nitrogen metabolism possibly linked to nitrogen assimilation.

Cluster 3 shows enrichment in fatty acids degradation and folate biosynthesis. 


\subsubsection{Transcriptional landscape of Tetradesmus obliquus slm1}

The previous section described transcriptional adaptations in a 16:8 h LD cycle growth condition of $T$. obliquus wild type. In this section we compare changes in gene expression between the starchless mutant $(s / m 1)$ and wild-type to understand the differences resulting from the lack of starch accumulation. An overview of gene expression data in $s / m 1$ in comparison to the wild-type is shown in Figure 3.1C. The PC analysis shows very similar regulation over time between both strains. While many time points display similar expression patterns, there are also clear differences related to the separation in time phases.

The s/m1 time points from phase I seem to have an earlier change in expression in comparison to the same time points in the wild-type. The overall expression profile from phase I until $3 \mathrm{~h}$ is very similar between the wild-type and $\operatorname{s} / m 1$ but with a minor advance over PC1 for $s / m 1$. The $s / m 1$ samples at $6 \mathrm{~h}$ and $9 \mathrm{~h}$ are displaying very similar expression to the wild-type on the PC1, but distinctly different on the PC2. This change reflects a significant earlier change of expression of genes that contribute to PC2 and causes $\operatorname{sim} 1$ samples to be in phase IIb. Finally, the processes related to the changes in phase III seems to linger in comparison to the wild-type. This is, the $\operatorname{sim} 1$ time points at $13 \mathrm{~h}$ display similar expression on the PC2, but did not yet reach the expression of the wild-type in PC1. The samples at $16 \mathrm{~h}$ are getting closer to the wild-type, but still seem to display a small delay in the changes of their expression profiles. As for the wildtype, the PC analysis considering the whole set of expressed genes displays a very similar pattern, but even noisier than the PC analysis of selected gene set described above.

Figure 3.1D shows expected similarities between time points, but the biological duplicates from 6-9 $\mathrm{h}$ and 13-16 $\mathrm{h}$ appear more similar than in the wild-type, which agrees with the PC analysis plot. These similarities reflect the described earlier change and delay in the associated processes. The relative time phases of $\operatorname{sim} 1$ are also shown in Figure 3.1E. Overall, two main trends are observed: earlier changes in expression of processes shortly after light and a delay in change of expression of the processes before the dark period. 


\section{Comparison of the gene expression dynamics in $T$. obliquus wild-type and $s / m 1$}

Using the regression based approach of maSigPro, we identified genes with differences in expression between $\operatorname{sim} 1$ and wild-type. Our experimental design included sequencing samples of the wild-type every hour and of $\operatorname{sim} 1$ every three hours. However, maSigPro is designed to allow uneven distribution of time points. 784 genes showed no significant differences in their expression profile between both strains, while 3,902 genes showed significant differences between them. Additionally, 40 genes were found to have a time profile in the mutant, while they did not have a time profile in the wild-type. This could mean that those genes were too noisy or were not expressed in the wild-type.

\section{Differences in time profiles}

Clustering was done for the gene expression data obtained from the mutant s/m1. Analysis of clustering performance indicators again resulted in 6 clusters. As previously stated, 784 genes did not show any significant difference in time dynamics between the two strains, therefore, these genes were used to guide the identification of the mutant clusters. The cluster assignments of these 784 genes in both strains is shown in Figure 3.3A. The wild-type genes in clusters 1 and 2 are all found in one $\operatorname{sim} 1$ cluster, named cluster $1 \& 2$. Likewise, the wild-type genes in clusters 3 and 5 are all found in one $\operatorname{s} / m 1$ cluster, named cluster $3 \& 5$. The wild-type genes in cluster 4 and 6 ended up in separate $\operatorname{sim} 1$ clusters and consequently kept the same cluster name. Finally two new clusters became apparent, termed $A$ and $B$.

Figure 3.3B shows the cluster assignments of the 3,902 genes with significant differences in their time profiles when comparing the wild-type and the s/m1. From the genes with altered expression (Figure 3.3B), there is a general trend of genes to remain clustered together. The wild-type genes in cluster 2 are mainly found in the associated slm1 gene cluster 1\&2. Similarly, almost all wild-type genes of cluster 6 are also found in the associated $\operatorname{sim} 1$ gene cluster 6 . The wild-type genes in cluster 4 are distributed over five $\operatorname{s} / m 1$ gene clusters, with the majority in the associated $\operatorname{sim} 1$ cluster 4 . The wild-type genes in cluster 3 are distributed over three clusters, with the majority in the 
associated $\operatorname{s} / m 1$ cluster $3 \& 5$. Interestingly, most of the wild-type genes in cluster 1 end up in $\operatorname{s} / m 1$ cluster $B$, while a substantially lower amount of genes end up in $\operatorname{sim} 1$ cluster 1\&2. Similarly, the genes in wild-type cluster 5 are distributed over $\operatorname{sim} 1$ cluster $3 \& 5$, 5 , and the majority in $\operatorname{s} / m 1$ cluster A.
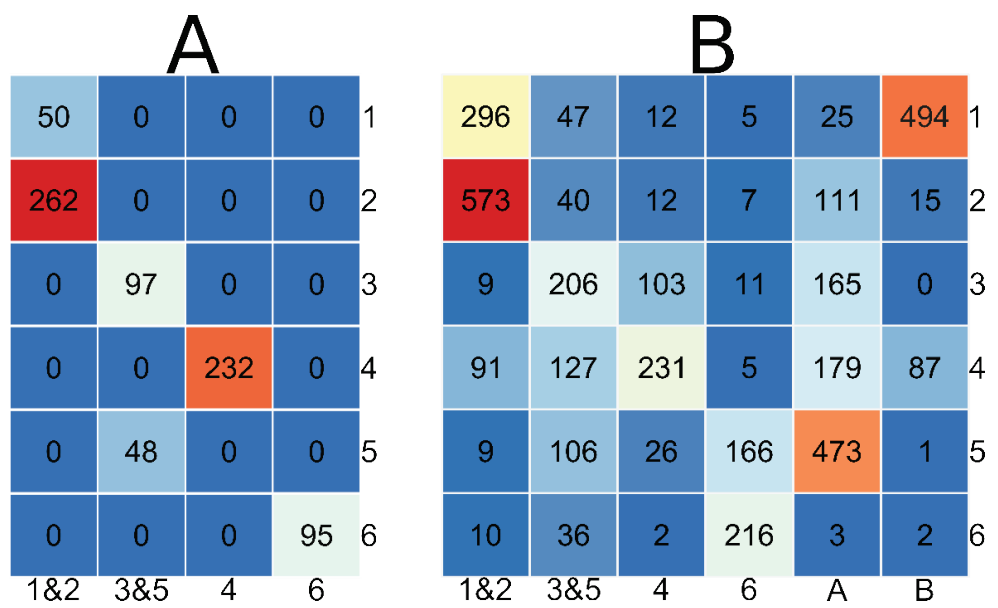

Figure 3.3. Distribution of genes between time profile clusters of Tetradesmus obliquus wild-type (rows) and starchless mutant slm1 (columns). (A) 782 genes with the same time expression profile in slm1 and wild-type. (B) Genes with significantly different time profiles in $s / m 1$ and wild-type, as identified by maSigPro (3,902 genes). Background color ranges from blue, white, to red, with red being the highest value and blue the lowest.

SIm1 cluster profiles are depicted in Figure 3.4, together with the associated wild-type clusters. Overall, the time profiles of $\operatorname{s} / m 1$ clusters show strong similarities with the associated wild-type cluster time profiles. SIm1 clusters A and B are displayed together with the wild-type clusters 5 and 1, respectively, due to the large number of genes found in common. The profile of $\operatorname{sim} 1$ cluster $1 \& 2$ is similar to that of wild-type clusters 1 and 2, but appears shifted and lacks strong peaks (Figure 3.4A). The genes that were found in wild-type cluster 4 and are now found in $\operatorname{sim} 1$ cluster $1 \& 2$, are thus expressed earlier in the diurnal cycle for the mutant as compared to the mutant. This could however be due to the difference in the sampling frequency, that may have resulted in missing the real peak time. The profile of $\operatorname{sim} 1$ cluster $3 \& 5$ is also similar to the profiles of wild-type clusters 3 and 5 , as its expression is highest during the night and first hours of the day (Figure 3.4D), and low during the rest of the day. Expression of 
genes in the $\operatorname{s} / m 1$ cluster $3 \& 5$ does not increases before the dark, and that it does not decrease before the beginning of the light period. Profiles of $\operatorname{sim} 1$ cluster 4 and 6 look more alike to their corresponding wild-type profiles, but with a visible lower expression during the light and dark period (Figure 3.4B and E). Notably, a substantial number of genes of the wild-type cluster 5 end up in $\operatorname{sim} 1$ cluster 6 , meaning that they are probably not expressed during the dark period anymore in s/m1. SIm1 cluster A (Figure $3.4 \mathrm{C}$ ) is a new cluster with a single peak at $13 \mathrm{~h}$ and low expression during the night and beginning of the day. Most genes from wild-type cluster 5 end up in this new cluster. To a lesser extent, genes from wild-type cluster 2,3 and 4 were also found in this cluster. This means that all these genes are no longer expressed in the dark for $s / m 1$. This is probably the most drastic change of expression between the strains. The time profile of $\operatorname{s} / m 1$ cluster B (Figure 3.4F) is most similar to wild-type cluster 1 , from which it gets most genes. It is also like wild-type cluster 1 and 4 with a slight shift of the peaks. Thus the transfer of genes from wild-type clusters 1 and 4 to $\operatorname{s} / m 1$ cluster $B$ reflects an earlier change of expression of these genes. Again, the lower sampling resolution can affect the outcome in this case.

In summary, we notice that most changes in expression were not associated to different cluster in the wild-type and $s / m 1$. These genes should be more affected on their amplitude, and not necessarily on the time regulation. There were also a number of genes associated to different cluster in the wild-type and $\operatorname{slm} 1$. These were subjected to strong changes in time regulation, which in most cases resulted in a time shift. Overall, many cases of detected changes could be the consequence of reduced sampling resolution in $s / m 1$. Among the genes with altered time regulation in $s / m 1$, there is a large number of genes not being expressed during the dark anymore. 

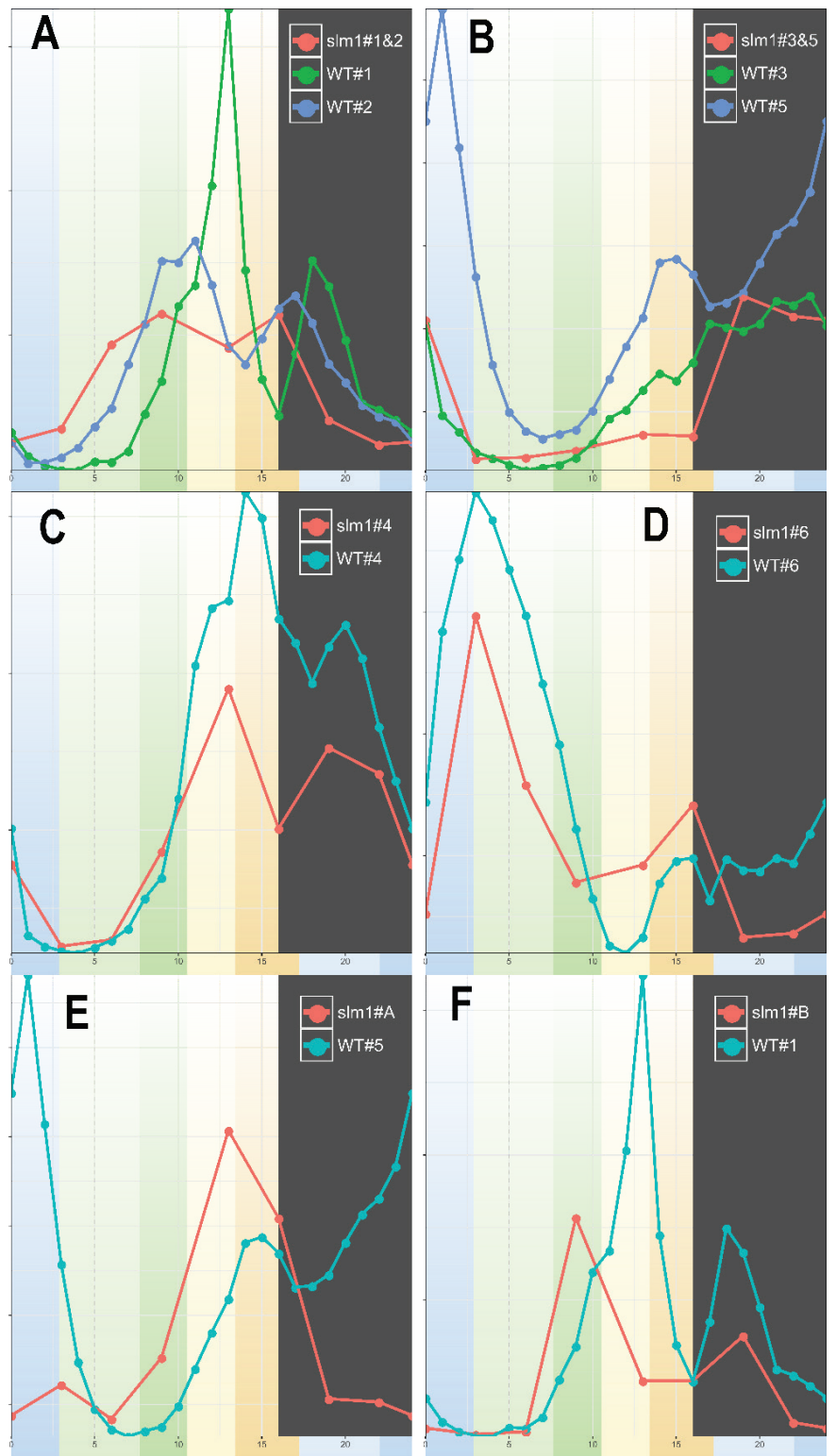

Figure 3.4. Expression profiles of clusters obtained from Tetradesmus obliquus slm1 samples and their associated profiles in the wild-type. Wild-type clusters 1 and 2 , $s / m 1$ cluster $1 \& 2$ (A); wild-type and $s / m 1$ cluster 4 (B); wild-type cluster 5 and $s / m 1$ cluster A (C); wild-type clusters 3 and 5 , slm1 cluster 3\&5 (D); wild-type and slm1 cluster 6 (E); wild-type cluster 1 and $\operatorname{slm} 1$ cluster B (F). Slm1 clusters were plotted together with the related wild-type clusters according to the distribution of genes in Figure 3.3. Background colors refer to the time phases identified in the wild-type and depicted in Figure 3.1E. 


\section{Functional differences between gene clusters in T. obliquus wild-type and $s / m 1$}

As shown in Figure 3.3.B, the genes were transferred in rather large groups from wildtype clusters to $\operatorname{s} / m 1$ clusters. Furthermore, the $\operatorname{s} / m 1$ clusters associated to wild-type clusters also conserved a majority of genes. As a result, the enrichments remain very similar and are in agreement with the observed gene transfer shown in the Figure 3.3B. $S / m 1$ clusters $A$ and $B$, are similar to wild-type clusters 5 and 1 , respectively in terms of enrichment. $\operatorname{S} / m 1$ cluster $1 \& 2$ remains enriched for glutathione metabolism and the pathways related to carbohydrates, but not for the two amino acids pathways (lysine degradation and phenylalanine metabolism). This cluster also remains enriched in cellular structure and DNA replication, but without any biological process GO terms. SIm1 cluster $3 \& 5$ remain enriched in fatty acid degradation and several amino acids metabolisms, but no other fatty-acids related pathways are enriched in this cluster. Enrichments related to gene expression, amino acids synthesis, and protein translation activities, seems to have been transferred from wild-type cluster 5 to $\operatorname{s} / m 1$ cluster A (Figure 3.3B). Glutathione metabolism is also found enriched in $\operatorname{sim} 1$ cluster A and those genes were found in wild-type cluster $4 . \operatorname{SIm} 1$ cluster 4 remains enriched in pathways related to carbohydrate metabolisms but no more in nitrogen metabolism, which is not enriched in any $\operatorname{s} / m 1$ cluster. Just like the time profile, the enrichment in slm1 cluster 6 is very similar to the one of wild-type. Again, due to the high number of genes from wild-type cluster 1 and the few from wild-type cluster 4 , the enrichment in slm1 cluster $B$ is comparable to wild-type cluster 1 , and retinol appears to be transferred from wild-type cluster 4. Globally, the enrichments are following the expectations drawn from the gene transfers described in Figure 3.3B. The enrichments allow us to track the changes in cellular phenotype.

\subsection{Discussion}

This study analyzed gene expression dynamics in a 16:8 h light/dark (LD) cycle synchronized culture of Tetradesmus obliquus wild-type. Hourly sample collection and RNA sequencing allowed us to observe in detail the changes of gene expression during a diurnal cycle. A starchless mutant of the same species (s/m1) was also studied. SIm1 
was cultivated in the same conditions, but RNA samples were taken every three hours. The succession of cellular events were then compared between the wild-type and the slm1 mutant, allowing us to examine the role and importance of starch as a transient energy storage compound in this microalga.

\subsubsection{The diurnal rhythm of $T$. obliquus wild-type is driven by six transcriptional phases}

The overall analysis of the changes in gene expression in both the wild-type and $\operatorname{sim} 1$ strain shows a circular pattern in the PC analysis plot. This analysis established two strong principal components. In the wild-type, changes in expression appeared to occur sequentially, back and forth along each PC. In the studied process, two main effects may impact gene expression being light availability and time itself. It is important to stress here that in our experimental setup, $T$. obliquus cells are synchronized to the diurnal LD cycles (León-Saiki et al., 2017). Many organisms synchronized their metabolism to anticipate the changes in the environment (Mittag, 2001; McClung, 2006; Causton et al., 2015). For photosynthetic microorganisms, this synchronization provides a benefit as they can capture sunlight efficiently during the day and perform light sensitive processes at night (Suzuki and Johnson, 2001; de Winter et al., 2013). In Tetradesmus obliquus synchronization was observed in growth and cell division, as well as in changes in biomass composition (León-Saiki et al., 2017).

\subsubsection{Time phases}

The overall expression patterns and the similarities between time point samples in the wild-type lead to the identification of 3 major and 3 more subtle shifts of expression at specific time points, which separates the 6 time phases described in Figure 3.1E. These time phases can also be associated to changes along the two first PC. The differences between phase I and phases II and III are the most prominent. In the PC plot these phases are mainly separated along the first PC, that represents $74 \%$ of the total variance of the wild-type data.

The temporal structure represented by the identified time phases is also reflected in the expression patterns of the gene clusters. The identified clusters in the wild-type 
show distinct peaks of expression following a strict succession. Thus, cluster 5 peaks during phase la; cluster 6 during Ila; cluster 2 during IIb, cluster 1 during IIla, cluster 4 during IIIb, and, finally, cluster 3 during Ib. It should be stressed that the choice of 6 phases and 6 clusters was done in a totally independent way. Thus, the agreement between both indicates that they are related to each other.

\section{Temporal succession of cellular events as described by the gene clusters}

An overview of the changes in the transcriptional landscape and experimental measurements of the wild-type through the diurnal LD cycle is presented in

Figure 3.5.

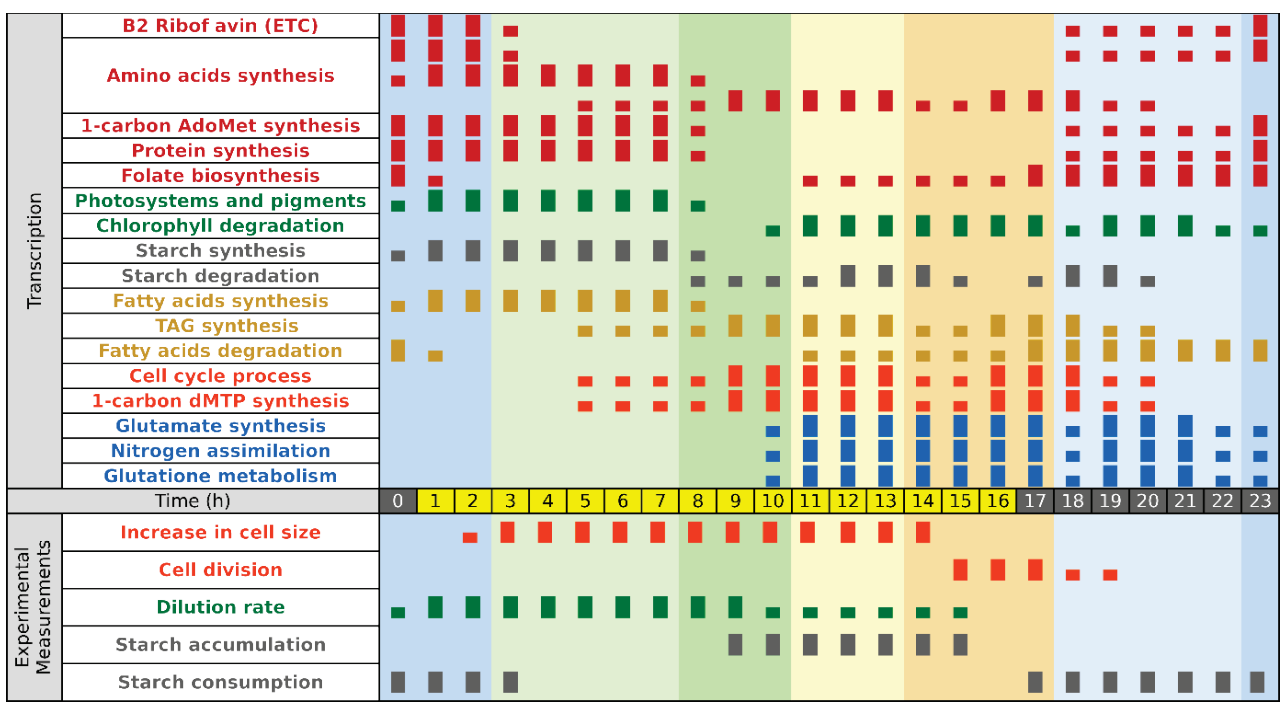

Figure 3.5: Overview of the diurnal changes in the transcriptional landscape and experimental measurements of Tetradesmus obliquus wild-type under 16:8 h light/dark cycles. The experimental measurements were taken from León-Saiki et al. (2017). Small and big rectangles indicate that the process is occurring at low or high level, respectively. Groups of related processes are indicated in the same color.

The median profile of the genes in cluster 5 peaks the first after light. The enrichment of cluster 5 reflects some preliminary metabolic processes amino acids synthesis and protein synthesis. At the same time, enrichment in riboflavin metabolism indicates that the cellular respiration is initiated, energy is retrieved from catabolism of starch or 
possibly fatty acid degradation. This is in agreement with the observed use of starch at the beginning of the day. The small peak of expression found around $15 \mathrm{~h}$ is probably a result of requirements for cellular division and night processes. Other amino acids synthesis pathways are also found enriched in different clusters, but cluster 5 contains the majority. There is a significant number of metabolic processes that contains a mixture of genes regulated as in cluster 5 and 6 , such as the carbon fixation.

Next, cluster 6 mainly contains genes involved in pigment and in starch synthesis, besides carbon fixation and amino acids synthesis. The main contributing genes in the PCA (Figure 3.1A) were also found in cluster 6. These main contributors are the light harvesting complexes, which are known to display a large variation in transcription. As displayed in Figure 3.5, the overall transcription increase of the genes related to pigment synthesis and the photosystems correlates directly with the sharp increase in dilution rate at the beginning of the day as observed by León-Saiki et al. (2017). The pathway enrichment did not reveal starch and sucrose metabolism because only four enzymes involved in synthesis are present in the pathway map (Supplementary Figure S3.1 and S3.2). Three out of the four enzymes for starch synthesis are found in this cluster. This agrees with the experimental observation of a net use of starch in the first hours of the light period. Then, when starch synthesis is upregulated (early day) and enzymes are translated, starch synthesis starts and we see net synthesis of starch from $5 \mathrm{~h}$ after the light went on. More details are given below in a dedicated section on starch metabolism.

Following cluster 6 , the genes in cluster 2 show a peak in expression at $13 \mathrm{~h}$. This cluster contains genes involved in the whole cell cycle and some protein maturation. There seems to be an important level of protein maturation through glycosylation in the endoplasmic reticulum at this stage. The maturation process is compatible with the observed cell growth. Furthermore the GO enrichment shows terms associated with progression through the cell cycle, meaning that at this time, DNA replication starts and takes place in the following hours. Finally, GO terms on organelle fission, chromosome organization, and organelle organization, point towards cell division which agrees with the observed cell division that starts just before the night and continues for a main part during the early night. 
Shortly after the genes in cluster 2 peak, genes associated to cluster 1 increase in expression. This cluster contains genes related to degradation of various glycans together with enrichment in several carbohydrate metabolic pathways. The information on individual genes indicates that these reactions are taking part in the starch degradation. Starch is degraded during the night, which is thus in agreement with the expression of these genes just before the start of the night in cluster 1 . Since the genes are already upregulated before the dark period starts, this is also suggests that there is a circadian rhythm signaling that the night is coming. Furthermore the large diversity of enrichment in carbohydrate related pathways is suggesting interconversion between carbohydrates. Additionally, it is possible that the interconversion of sugars is related to the synthesis of cellobiose that is needed for the growing cell walls of the dividing cells.

The next cluster for which the gene expression shows a peak is cluster 4 . Cluster 4 also shows enrichment for carbohydrate pathways, but more importantly it is enriched in glycolysis. Together, they clearly indicate that the genes for starch degradation and glycolysis are providing the means for the organism to generate the needed energy during the night. Glutamate and ATP synthesis associated genes are strongly upregulated in cluster 4 . It seems that the wild-type overexpresses these genes to continue assimilation of nitrogen during the night that can be used for the synthesis of chlorophyll and amino acids at the start of the day. In this way new photosystems can be quickly synthesized and the light can be harvested efficiently. This is in agreement with the observed nitrogen consumption during the night by the wild-type. Notably, s/m1 does not consume nitrate in the night and nitrogen metabolism is not enriched for $\operatorname{sim1}$, which suggests that pathways in nitrogen metabolism are regulated by the energy status of the cell. Nitrate reductase was not annotated and we could not analyze its expression. Nevertheless, the nitrite reductase was not regulated in time and its expression was extremely low. Cluster 4 also shows an overall increase of expression in genes associated to the glutathione reductive cycle, indicating some form of oxidative stress. This stress could be a side effect of the whole day exposure to light leading to accumulation of reactive oxygen species.

Finally, the next cluster showing an increase in expression is cluster 3. This cluster is enriched in fatty acid degradation. Apparently, degradation of fatty acids occurs during the night and early morning, according to the regulation of genes in cluster 3 . 
Degradation of fatty acids in the night is, however, not observed experimentally. Possibly, these enzymes are involved in remodeling of membranes that has to occur after cell division.

In summary, the annotation and enrichment analyses uncovered the general gene function of the genes in each cluster, thus showing a clear succession of cellular events. Overall, the gene clusters represent the following general functions, by order of highest peak appearance: RNA transcription, gene expression, protein synthesis, pigments synthesis, starch synthesis, fatty acids synthesis, protein glycosylation, cellular growth and division, starch degradation and sugars inter-conversions, riboflavin synthesis, folate synthesis, and, finally, degradation of fatty acids (Figure 3.5). This succession of transcriptional events and their relative expression in time indicates that $T$. obliquus does not simply adapt its cellular phenotype to direct signals like the switching on and of the light, but it can regulate the gene expression in anticipation of changes from light to dark and vice versa. For example, before the light is turned on, the microalga starts synthesizing important metabolites that will fulfill the high demand of gene expression and protein synthesis and allow quick synthesis of photosystems to efficiently capture the light. Likewise starch degrading enzymes are upregulated before the night starts.

\subsubsection{Impact of the lack of starch on the diurnal rhythms}

When analyzing data from s/m1, significant changes in the temporal profiles were identified for the vast majority of the genes compared to the wild-type (3,902 genes). In most cases, the genes remained clustered together, as is for the wild-type, and most differences can be explained by a time shift or a change in amplitude of the peaks. Time changes of expression in phase II and IIla contain most of the overall differences between the wild-type and $s / m 1$. However, the less frequent sampling of $s / m 1$ (every $3 \mathrm{~h})$ might have impacted the detection of expression changes and the cluster separation.

The lack of starch seems to have other repercussions than just a time shift in the processes. From the six clusters for the wild-type, two clusters (1-2 and 3-5) could not be efficiently separated in s/m1. However, two extra clusters of time profiles were 
found: A and B. Whereas cluster B could be confounded with wild-type cluster 1, cluster A showed a novel expression profile not seen in the wild-type.

The biochemical data for the $\operatorname{sim} 1$ also matches the transcriptomic results, but to a lesser extent than for the wild-type. Analysis of data obtained from the starchless mutant $\operatorname{s} / m 1$ shows that the phases from the wild-type are to some extent preserved. However the mutant shows an earlier change in expression of processes expressed in the morning and an extension of the change in expression of processes expressed shortly before the dark period.

The lack of starch seem to result in rescheduling some biological processes during the light period that could not happen at night due to the lack of energy. Processes required from expression and translation were transferred from cluster 5 to cluster $A$ in $\operatorname{s} / m 1$. Processes moved to $\operatorname{s} / m 1$ cluster $A$ are not expressed at night anymore. This means that the expression of these processes could be regulated by the energy status of the cell, which is still high when s/m1 cluster A peaks (before dark, Figure 3.4) and low during the night due to the lack of starch. For example, thiamine metabolism is found enriched in cluster $A$, which is a coenzyme used in starch catabolism, indicating that starch might be used as an energy source. Additionally, in $s / m 1$, amino acids could be catabolized, as there is no starch metabolism enriched. Glutathione metabolism was found enriched in cluster $A$, but it does not reflect a strong change in expression compared to the wild-type in cluster 4 , besides the absence of expression during the dark period.

\subsubsection{Selected reactions and pathways}

In this section, we zoom in on a few specific pathways of interest.

\section{Starch synthesis}

While enrichment for "starch and sucrose metabolism" was associated to cluster 2, the detailed view at the pathway (supplementary Figure S3.1 and S3.2) revealed that the reactions causing the enrichment are related to starch degradation and sugars interconversions. More interestingly, three of the four necessary reactions for synthesizing starch are found enriched in cluster 6, being the ADP-Glucose 
pyrophosphorylase (EC:2.7.7.27), the starch synthase (EC:2.4.1.21) and the granulebound starch synthase (EC:2.4.1.242). The fourth, starch branching enzyme, was not found to be regulated in time. The granule-bound starch synthase was associated to another cluster in s/m1, but both strain profiles overlap and this difference appears to be due to the lower sampling resolution in the $s / m 1$. We also noticed that the expression of this enzyme peaks in the middle of the light period, few hours later than the starch synthase. The amylose isomerase (EC:2.4.1.18) was not found to be regulated in time for either strain.

The expression profile of processes related to starch synthesis and degradation show some correlation to the starch measurements (Figure 3.5). Starch is a transient energy compound and is subjected not only to daily turnover, but also to simultaneous synthesis and degradation (Stitt and Zeeman, 2012; León-Saiki et al., 2017). Between $16 \mathrm{~h}$ and $0 \mathrm{~h}$, only degradation occurs due to the lack of light energy for production. Between 0 and $4 \mathrm{~h}$, the starch synthesis machinery is regenerated along with many other energy demanding processes. During these first hours of light apparently the light reaction and carbon fixation still do not supply sufficient energy and carbon for the fast build-up of photosystems and there is still net degradation of starch, together with the newly formed 3-phosphoglycerate from the carbon fixation, to support metabolism. Between 4 and $8 \mathrm{~h}$, starch flow balances out after cluster 6 peaks. Between 8 and $15 \mathrm{~h}$, the starch production is at full potential and starch is accumulated. Right before dark, starch accumulation slows down due to the increased energy being used for cell division.

The ADP-glucose pyrophosphorylase is known to be the reason that $\operatorname{sim} 1$ cannot synthesize starch, and is due to a nonsense mutation resulting in a premature terminal codon in the small subunit of the protein complex (de Jaeger, 2015). Interestingly, in the same study that assessed the mutation, this gene was also found to be strongly down-regulated compared to wild-type (approximately 5 fold). Our cluster analysis associated this gene in cluster 6 for both strains. The expression of the gene encoding the small subunit (g788.t1) is much lower in $\operatorname{s} / m 1$ compared to the wild-type (Figure 3.6) and is even more reduced than previously observed (de Jaeger, 2015). As detected by the clustering method, the time profile appears similar. This conservation of regulation indicates no disturbances in the diurnal regulatory system for starch synthesis. Additionally, the large subunit of the ADP-glucose pyrophosphorylase 
(g12538.t1) is expressed similarly between both strains, with a potentially slight increase during light period. The sharp peak of expression at 14-15 h was not observed in $\operatorname{sim1}$, which may have been due again to the lower measurement resolution at those time points for s/m1. Finally, the small subunit is mostly expressed during the first half of the light period, and the large subunit is mostly expressed right before dark. This is a surprising gap in time between the synthesis of both subunits. Possibly, the large subunit is synthesized right before night in anticipation of the next light period, leaving more time for this larger protein to mature.

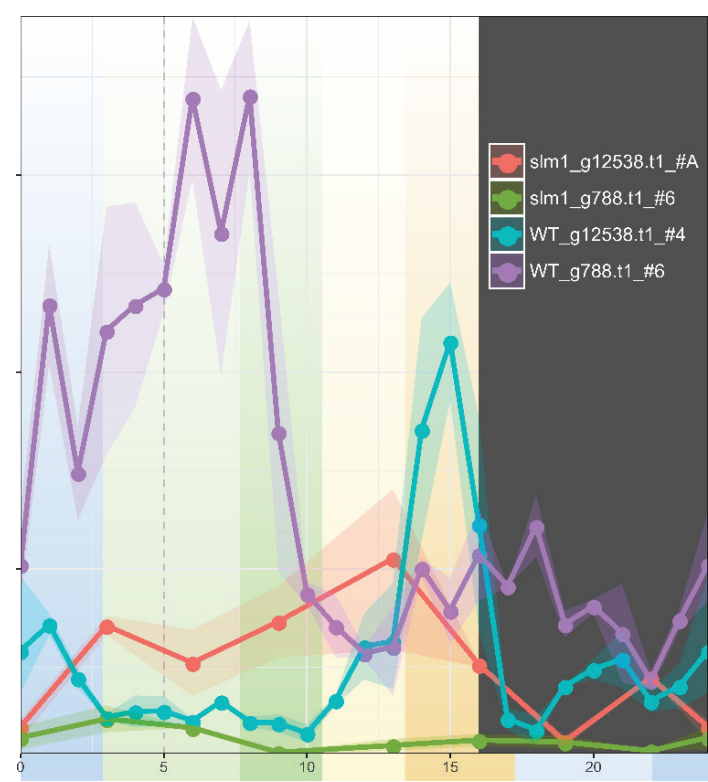

Figure 3.6. Changes in gene expression in the enzyme ADP glucose pyrophosphorylase (E.C. 2.7.7.27) large subunit (g12538.t1) and small subunit (g788.t1) for Tetradesmus obliquus wild-type and starchless mutant slm1. The line indicates the mean values, the ribbon around the line indicates the maximum and minimum values. Shaded area indicates the dark period. Background colors refer to the time phases identified in the wild-type and depicted in Figure 3.1E. 


\section{DNA and amino acids synthesis}

Folate, vitamin B9, is a vital cofactor necessary for diverse reactions and maintenance processes. Notably, folate activated one-carbons are used for synthesizing purines, thymidylate (dTMP) and are used in the methylation of homocysteine into methionine. dTMP is necessary for DNA synthesis and especially for cellular division. Methionine can be adenosylated (EC:2.5.1.6, not clustered) to a methyl donor Sadenosylmethionine (SAM-e), used in different processes such as protein methylation. The gene expression in the pathway "one carbon pool by folate" of the wild-type is clearly decoupled for these two processes. While dTMP related reactions are associated to cluster 2, SAM-e reactions are associated to clusters 5 and 6 . This result is also displayed in Figure 3.5, in which their respective synchronization with cellular division and protein synthesis is clear. Interestingly, "folate biosynthesis" was found enriched in cluster 3 (Table 3.1), together with the reactions from GTP until the precursor of dihydrofolate (DHF). Considering the temporal succession of these events, it seems that folate is being accumulated before the light period in order to sustain the high levels of protein synthesis at dawn.

\section{Nitrogen metabolism}

In $\operatorname{s} / m 1$, few genes were clustered in A instead of cluster 4, which had the consequence of losing the enrichment in nitrogen metabolism (Supplementary Figure S3.3 and S3.4). With the time profile plots of those reactions (Figure 3.4), we can confirm that the reactions kept a very similar expression pattern and intensity. Only the hydroxylamine reductase (EC 1.7.99.1) displays a change with significant decrease of expression at night, as expected from its association to cluster $A$. This also correlates with the lack of nitrogen consumption during the night in $\operatorname{sim1}$ (data not shown), probably because starch is needed to supply energy in the night for this process.

\subsection{Conclusions}

We show in this work that LD cycles induce systems level transcriptional patterns that indicate a strict succession of cellular events that match changes in biochemical parameters. Some of these adaptations appear to be a direct response to changes in 
light. However, in some cases, anticipation to the shift of light condition is observed, indicating a cellular time-keeping system.

Additionally, we studied the diurnal transcriptional changes in the starchless mutant of T. obliquus s/m1. The major change observed in the timing of expression of different processes was that expression during the night was not anymore upregulated for a number of processes. These were processes related to nitrogen assimilation and protein synthesis. This shows that these pathways are somehow regulated by the availability of energy. Furthermore, these processes have to be done now during the day, meaning that $\operatorname{sim} 1$ is less prepared to start growing when the light goes on. Additionally, we observed that the mutation rendering $\operatorname{s} \mid m 1$ unable to produce starch impacts pathways directly associated to energy storage, such as carbon fixation. Insights in these processes are very valuable to develop strategies to increase yields. In s/m1, there is additionally an overall earlier onset of processes triggered shortly after the light is on and an extension of the time length of processes that would normally occur before the beginning of the dark period. The lack of starch as transient energy storage forces energy demanding processes to occur during the light period. This indicates that no other transient energy storage compound is available for the cell.

Lastly, we show that there is a general good agreement between the transcriptional changes and the changes in the external phenotypes, as characterized by experimental measurements. This study shows that a single change in a metabolic pathway due to mutations or metabolic engineering can result in changes in the timing of expression of biological processes.

\section{Funding}

This research project was supported by the Consejo Nacional de Ciencia y Tecnología CONACYT, Mexico, Scholar 218586/Scholarship 314173. In addition, GMLS is part of the program "Doctores Jóvenes para el Desarrollo Estratégico Institucional" by the Universidad Autónoma de Sinaloa.

\section{Acknowledgments}

The authors would like to thank Tom Schonewille for the RNA extraction of the samples. 


\section{Supplementary information}

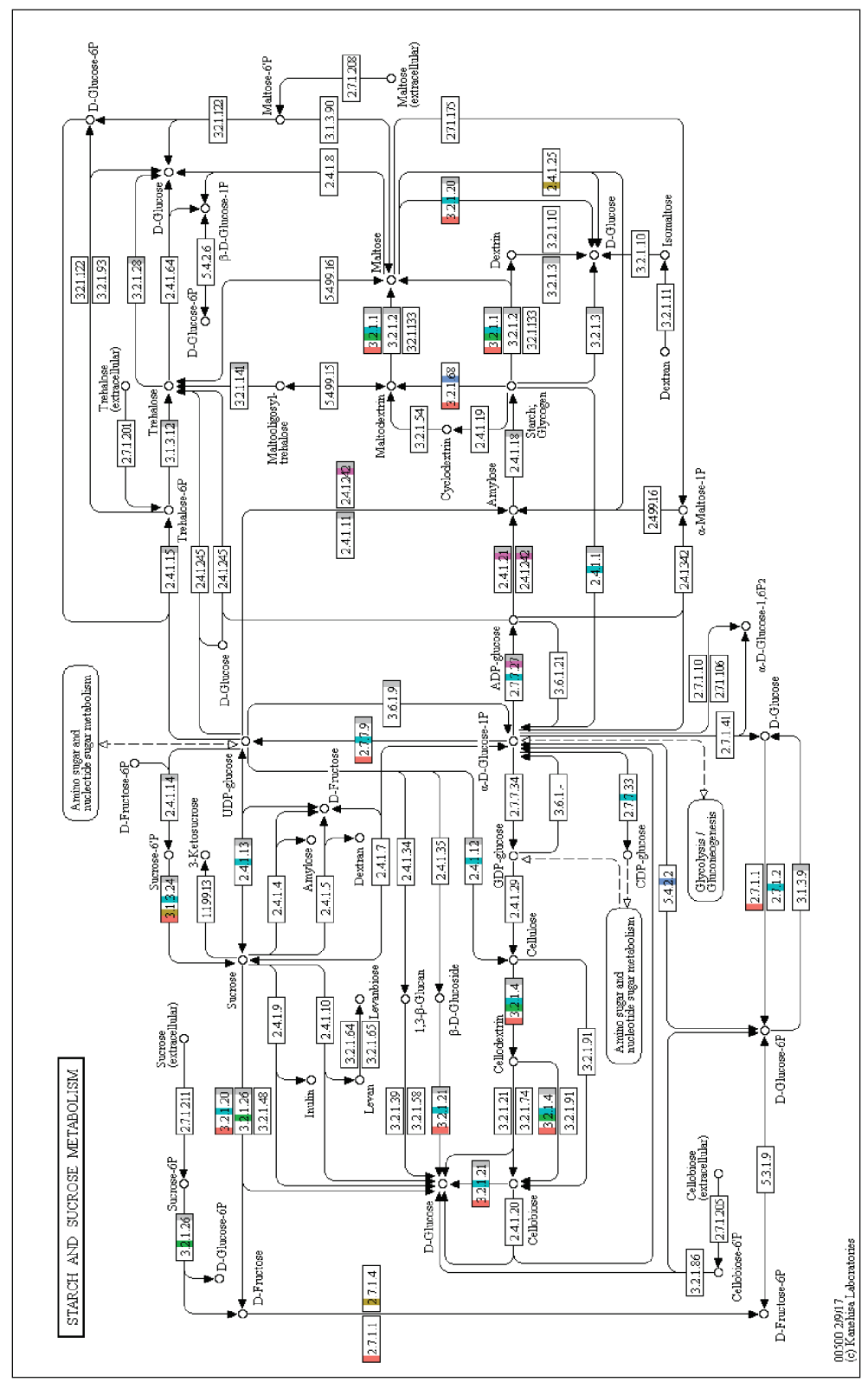

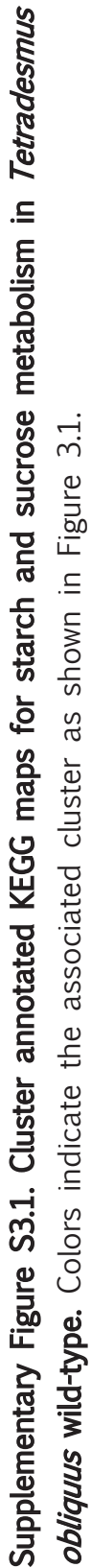




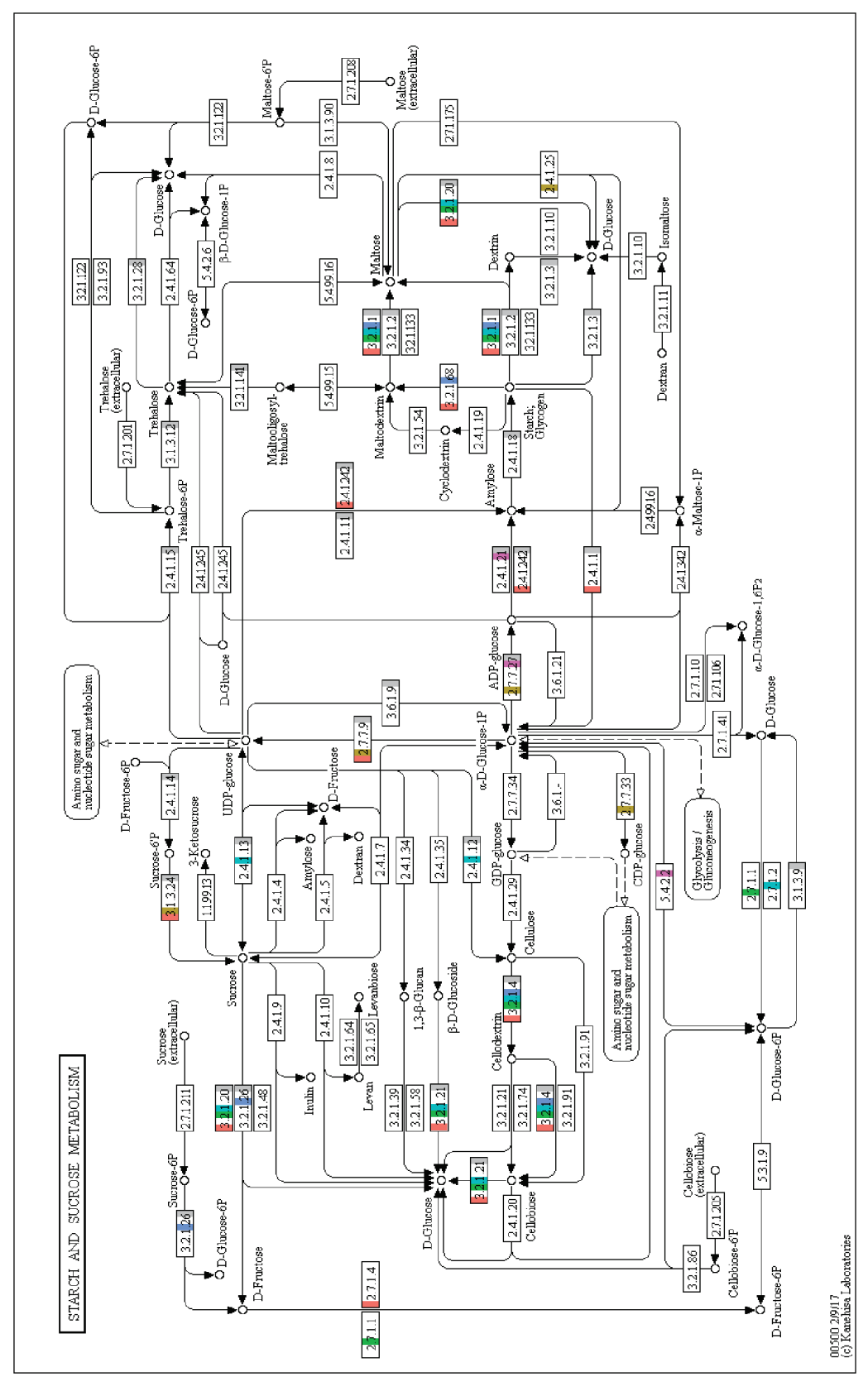

ญั

.드

틍

Ð

늘

ำ ๙

专 㐫

흔 ఫ্巳

농

ฮั

岃

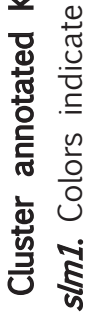

กู่

๒

bo

농 


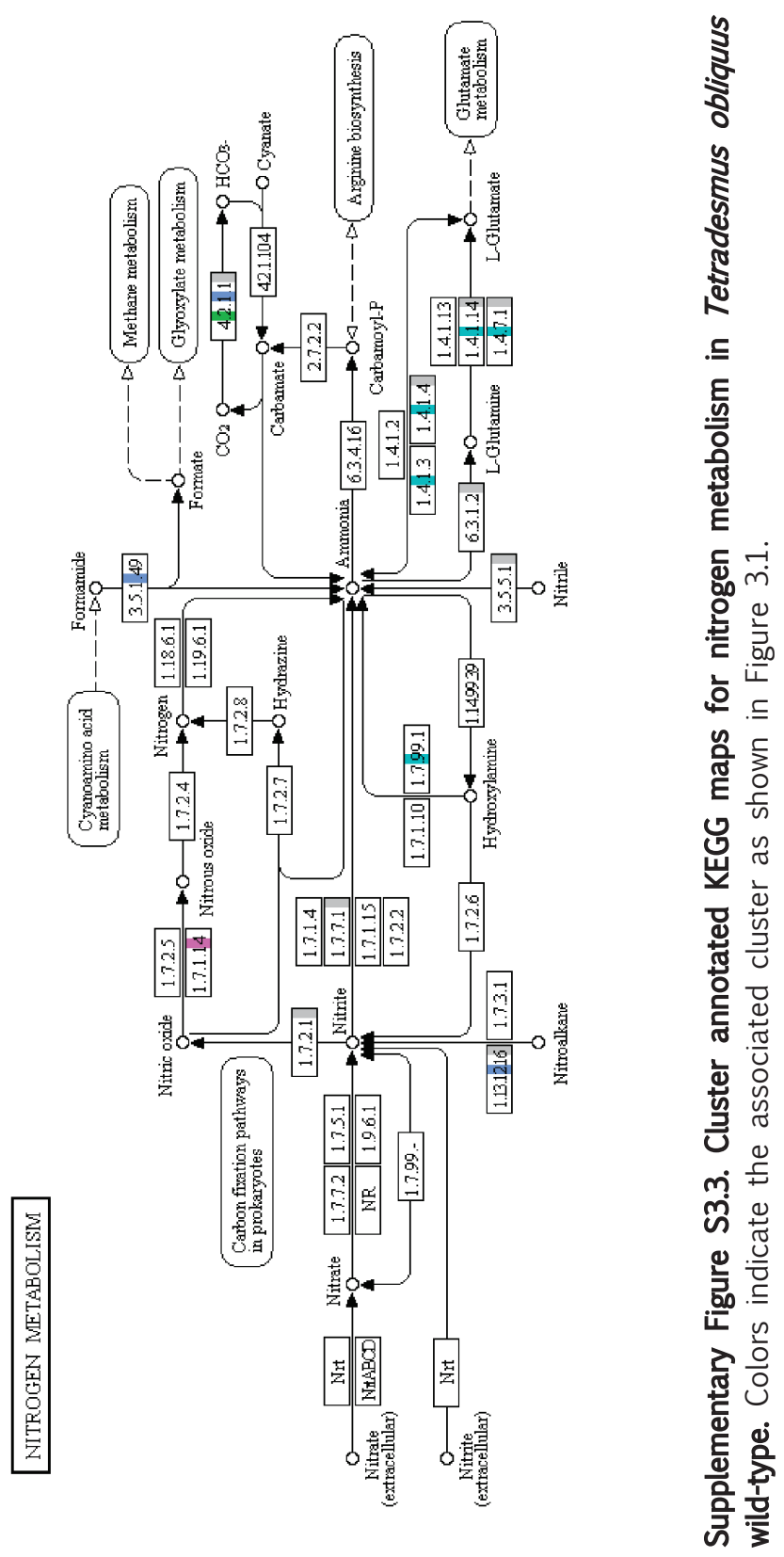




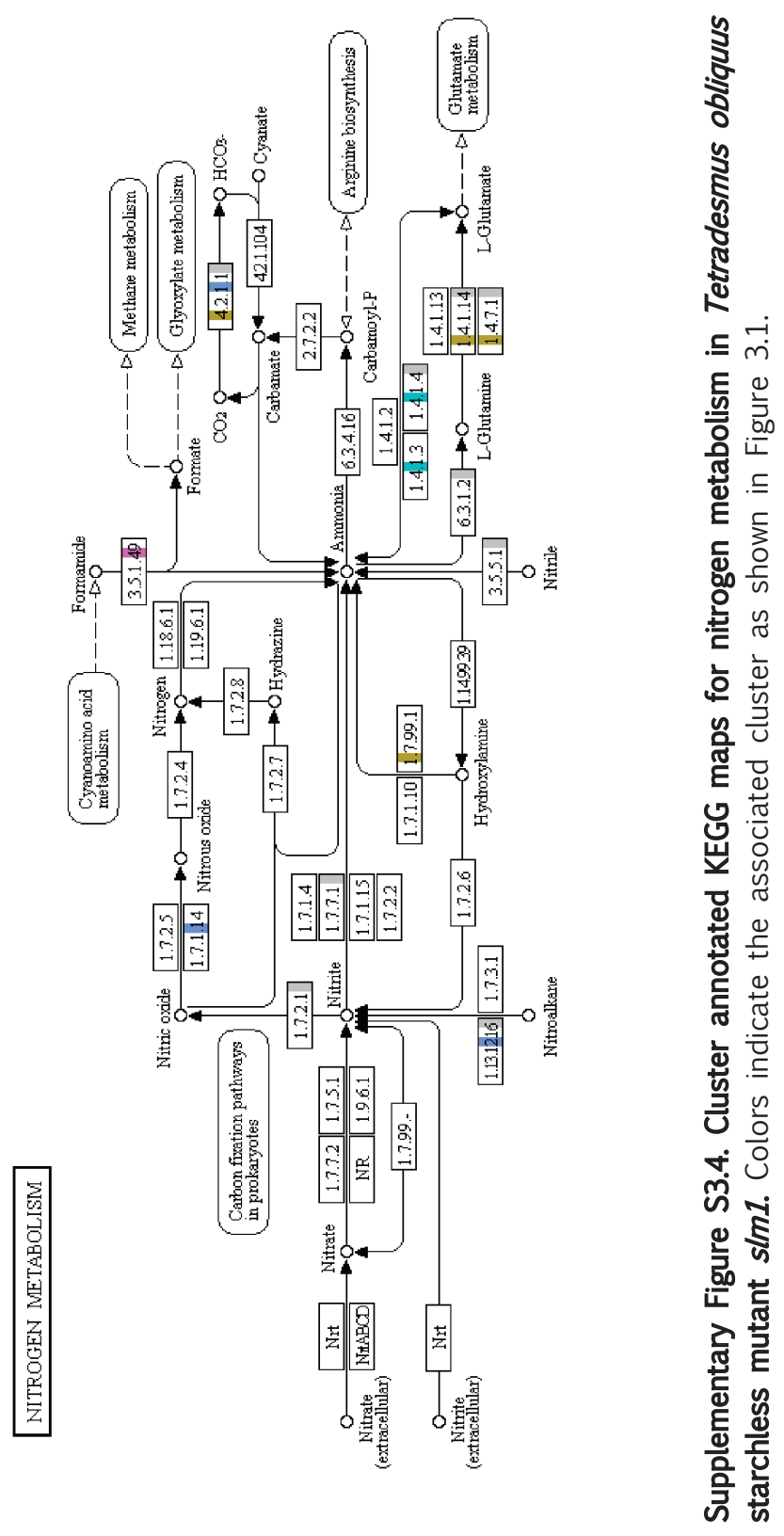




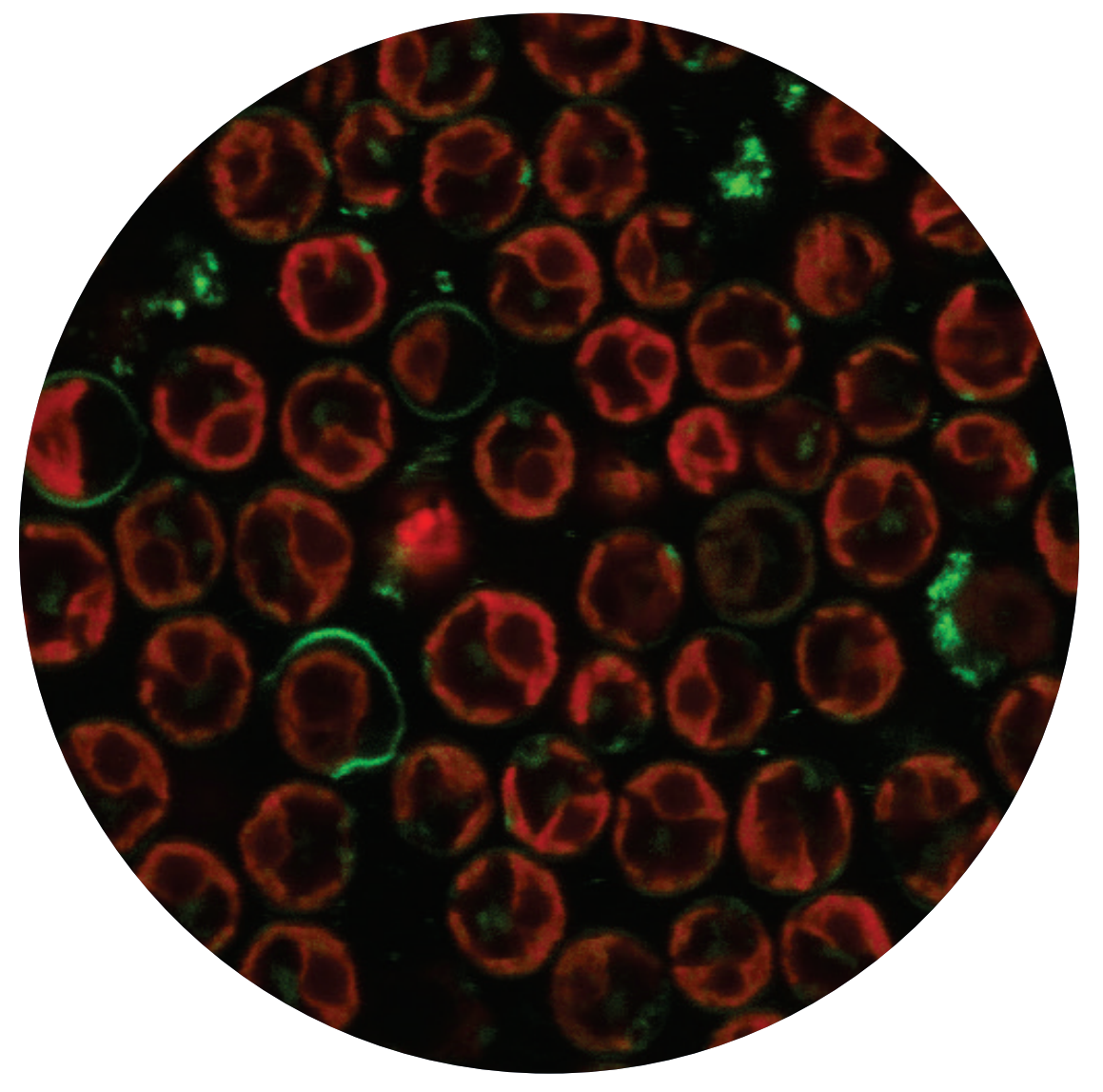




\section{Chapter 4}

\section{The impact of day length on cell division and efficiency of light use in a starchless mutant of Tetradesmus obliquus}

This chapter is published as:

GM León-Saiki, T Cabrero Martí, D van der Veen, RH Wijffels, DE Martens (2018) The impact of day length on cell division and efficiency of light use in a starchless mutant of Tetradesmus obliquus. Algal Research 31, 387-394 
Large scale microalgal production will be primarily done under natural sunlight conditions, where microalgae will be exposed to diurnal cycles of light and dark (LD) and to differences in the length of both periods (photoperiod). Tetradesmus obliquus (formerly known as Scenedesmus obliquus), a strain with potential for biofuel production, and the starchless mutant s/m1 were grown under 3 different LD periods: 16:8 h, 14:10 h and 12:12 h. Cell division started a fix number of hours after the light went on ('sunrise'), independently of the length of the photoperiod. For the wildtype, cell division started approximately $14 \mathrm{~h}$ after the beginning of the day and occurred mainly at night. For the starchless mutant $\operatorname{s} / m 1$, timing of cell division was also independent of the photoperiod length (starting 10-12 h after 'sunrise'). However, as opposed to the wild-type, cell division always started during the day. For both strains, growth rate increased with increased length of the light period. The $s / m 1$ mutant is capable of surviving long dark periods (up to $12 \mathrm{~h}$ ) despite the lack of starch. In general, the $\operatorname{sim} 1$ mutant has a lower photosynthetic efficiency than the wild-type, with the 12:12 h LD resulting into even less efficiency than the other two LD cycles. 


\subsection{Introduction}

Microalgae can be used as source for commercial products of interest such as biofuels, chemicals, food, and feed (Wijffels et al., 2010; Wijffels and Barbosa, 2010). Large scale microalgal production will be primarily done under natural sunlight conditions (Norsker et al., 2011; Blanken et al., 2013), where microalgae will be exposed to diurnal cycles of light and dark (LD). Diurnal LD cycles are ubiquitous and many organisms synchronize their metabolism to anticipate the changing environment (Shearman et al., 2000; Mittag, 2001; McClung, 2006; Salichos and Rokas, 2010; Causton et al., 2015). Environmental cues (known as Zeitgeber, which is German for time indicator) entrain the internal timing to a period of $24 \mathrm{~h}$ (McClung, 2006; Niwa et al., 2013). Cues such as sunrise (dawn), sunset (dusk), changes in light intensity or temperature, as well as light pulses can be used to entrain this diurnal cycle (Roenneberg and Foster, 1997). For photosynthetic organisms, synchronization to the diurnal LD cycle translates into finetuning their photosynthetic apparatus to capture sunlight efficiently during the day and to schedule ultraviolet or oxygen sensitive processes (e.g. nitrogen fixation, DNA synthesis or cell division) at night (Nikaido and Johnson, 2000; Suzuki and Johnson, 2001; Fábregas et al., 2002; de Winter et al., 2013). In addition, the length of the light and dark periods under natural sunlight conditions varies depending on the region and the season, which has an impact on biomass productivity and photosynthetic efficiency (Jacob-Lopes et al., 2009) depending on the species.

The microalga Tetradesmus obliquus (formerly known as Scenedesmus obliquus (Krienitz and Bock, 2012) and reclassified as Acutodesmus obliquus (Wynne and Hallan, 2015)) is an industrially relevant strain whose potential has been demonstrated (Mandal and Mallick, 2009; Ho et al., 2012; Breuer et al., 2013b; Remmers et al., 2017). In addition, de Jaeger et al. (2014) developed a starchless mutant, s/m1, which is incapable of synthesizing starch due to a single nucleotide polymorphism in the small subunit of ADP-glucose pyrophosphorylase, the committed step of starch biosynthesis (de Jaeger, 2015). This mutant showed a higher maximum triacylglyceride (TAG) yield on light $\left(0.217 \mathrm{~g} \cdot \mathrm{mol}_{\mathrm{ph}}{ }^{-1}\right.$ compared to $0.144 \mathrm{~g} \cdot \mathrm{mol}_{\mathrm{ph}}{ }^{-1}$ for its wild-type) and a higher maximum TAG content ( $0.57 \mathrm{~g} \cdot \mathrm{gDW}^{-1}$ compared to $\left.0.45 \mathrm{~g} \cdot \mathrm{gDW}^{-1}\right)$ in batch cultures under nitrogen starvation (Breuer et al., 2014). Furthermore, the photosynthetic efficiency of the mutant was comparable to the wild-type under nitrogen starvation. 
Prior to the TAG producing step, which commonly takes place under nitrogen limitation/starvation and LD cycles, biomass must be grown under nitrogen replete conditions. Under nitrogen replete conditions, T. obliquus wild-type uses starch as a temporary energy storage compound during LD cycles (León-Saiki et al., 2017). Thus, energy and carbon are stored during the day which are next used at night. When starch synthesis is blocked, as for starchless mutants, different effects on growth under nitrogen replete conditions are observed for different microalgae. However, most of the studies have been done under continuous light (Ramazanov and Ramazanov, 2006; Li et al., 2010b; Work et al., 2010; Vonlanthen et al., 2015), which is not relevant for outdoor production. Furthermore effects of the absence of starch are expected to be more severe during LD cycles, since the algae use starch during the dark as a source of energy and carbon. To our knowledge, only one report of growth of the starchless mutant of Chlorella pyrenoidosa STL-PI was done under 12:12 h LD cycles, showing an increase in growth compared to its wild-type (Ramazanov and Ramazanov, 2006). Additionally, as starchless mutants are made to improve TAG production, most studies on these mutants focus on their performance during the TAG producing step under nitrogen limitation or starvation conditions (Li et al., 2010b; Li et al., 2010a; Work et al., 2010; Breuer et al., 2014; Vonlanthen et al., 2015; Remmers et al., 2017), and little is known about their diurnal behavior under nitrogen replete conditions and LD cycles. The diurnal cycles of the starchless mutant of T. obliquus $s / m 1$ were studied under 16:8 h LD cycles, where this mutant showed synchronized growth and cell division even in the absence of starch or any other storage compound, albeit with decreased growth and energy efficiency compared to its wild-type (León-Saiki et al., 2017). However, as previously mentioned, production conditions outdoors will include variations in the light and dark periods and it is thus interesting to know how the mutant will react to these variations. Especially, it is interesting to know how the mutant will react to longer dark periods since a temporary energy storage compound is missing.

Therefore, the aim of this paper is to obtain insight into how a starchless mutant of T. obliquus copes with different LD periods as compared to its wild-type. For this, scheduling of cell division, energy efficiency and biomass composition were measured under 3 different photoperiods of typical day/night duration throughout the year for both the wild-type and the starchless mutant $s / m 1$. 


\subsection{Materials and methods}

\subsubsection{Strains, pre-culture conditions and cultivation medium}

Wild-type Tetradesmus obliquus UTEX 393 (reclassified from Scenedesmus obliquus (Krienitz and Bock, 2012) and Acutodesmus obliquus (Wynne and Hallan, 2015)) was obtained from the Culture Collection of Algae, University of Texas. The starchless mutant of T. obliquus (s/m1) was generated as described by de Jaeger et al. (2014). Liquid cultures of $100 \mathrm{~mL}$ of filter sterilized (pore size $0.2 \mu \mathrm{m}$ ) defined medium designed by Breuer et al. (2013b) were maintained in a culture chamber with shaker in $250 \mathrm{~mL}$ Erlenmeyer flasks $\left(25^{\circ} \mathrm{C}, 16: 8 \mathrm{~h}\right.$ light/dark cycles with $30-40 \mu \mathrm{mol} \cdot \mathrm{m}^{-2} \cdot \mathrm{s}^{-1}$, $150 \mathrm{rpm}$, air in headspace). Prior to the start of the experiments, cultures were placed in a shake incubator operating at $25^{\circ} \mathrm{C}$ with continuous light $\left(120 \mu \mathrm{mol} \cdot \mathrm{m}^{-2} \cdot \mathrm{s}^{-1}\right)$ and a headspace enriched with $2.5 \% \mathrm{CO}_{2}$ to reach the desired inoculation cell density.

\subsubsection{Reactor set-up and experimental conditions}

T. obliquus was continuously cultivated in a sterile flat panel airlift-loop reactor with a 1.7 $\mathrm{L}$ working volume and a $0.02 \mathrm{~m}$ light path (Labfors $5 \mathrm{Lux}$, Infors HT, Switzerland). Reactor set-up, temperature, $\mathrm{pH}$ and airflow were set and controlled as described by León-Saiki et al. (2017). Light was provided at an incident photon flux density of $500 \mu \mathrm{mol} \cdot \mathrm{m}^{-2} \cdot \mathrm{s}^{-1}$ in 3 different light/dark (LD) block cycles: 16:8 h, 14:10 h, and 12:12 h. Cultivations were turbidostat controlled, where the culture was diluted with fresh medium when the light intensity at the back of the reactor dropped below the setpoint $\left(10 \mu \mathrm{mol} \cdot \mathrm{m}^{-2} \cdot \mathrm{s}^{-1}\right)$. The feeding was stopped during the dark period to prevent washing of the culture.

The reactor was inoculated at an optical density $\left(O D_{750}\right)$ of 0.1 . Cultures were allowed to reach steady state, which was defined as a constant biomass concentration and $24 \mathrm{~h}$-dilution rate for a period of at least 3 residence times. After steady state was reached, liquid samples were freshly taken from the reactor and either immediately used for cell count $(1 \mathrm{~mL})$ and dry weight measurements $(3 \mathrm{~mL}$, in triplicate) or centrifuged for $5 \mathrm{~min}$ at $2360 \mathrm{x}$ for biochemical analysis $(12 \mathrm{~mL}$ for proteins, $5 \mathrm{~mL}$ for starch, $5 \mathrm{~mL}$ for triacylglycerides (TAG), and $5 \mathrm{~mL}$ for total carbohydrates). For biochemical analysis, the resulting pellet was transferred to bead beating tubes (Lysing 
Matrix E; MP Biomedicals Europe) or glass tubes (for total carbohydrates analysis) and stored at $-20{ }^{\circ} \mathrm{C}$. Pellets were freeze dried and stored again at $-20{ }^{\circ} \mathrm{C}$ until further analysis. Sampling was done in intervals of $1 \mathrm{~h}$ for cell counts. Biomass composition was analysed in intervals of $3 \mathrm{~h}$ for the 14:10 $\mathrm{h}$ and 12:12 $\mathrm{h}$ LD. For the 16:8 $\mathrm{h}$ LD, biomass composition was obtained from a previous publication (León-Saiki et al., 2017). In addition, at least 3 daily overflow samples were collected for each photoperiod and strain.

\subsubsection{Analyses}

Dry weight (DW) concentration was determined in triplicate as described by Kliphuis et al. (2012). Starch was measured using a total starch kit (Megazyme, Ireland) as described by de Jaeger et al. (2014) with the modification that $5 \mathrm{mg}$ of freeze dried biomass was used for the analysis. Protein content was measured using a colorimetric assay (Bio-Rad DC protein assay) as described by Postma et al. (2015) with the difference that 10-12 mg of freeze dried biomass was used for analysis. Triacylglycerol (TAG) content was determined as described by Remmers et al. (2017). Total carbohydrates were extracted and quantified according to DuBois et al. (1956) and Hebert et al. (1971).

\subsubsection{Cell number and size}

T. obliquus cells aggregate and form coenobia (Bišová and Zachleder, 2014). To separate the cells, a $1 \mathrm{~mL}$ cell suspension was sonicated on ice for $30 \mathrm{~s}$ at $30 \%$ amplitude using a probe sonicator (Sonics vibra-cell, USA). The absence of coenobia after sonication was verified under the microscope. Cell number and size were determined using a Beckman Coulter Multisizer 3 (Beckman Coulter Inc., USA). The sonicated culture was diluted 200 times with Isoton ${ }^{\circledR}$ II diluent solution. Cells with diameter above $2.5 \mu \mathrm{m}$ were counted. As some cell counts were done only in duplicate $(n=2)$, we show the range of values measured by including the maximum and minimum values found. 


\subsubsection{Dilution rate, doubling time and time-specific cell division rate}

Dilution rate $\left(D_{24 h}\right.$ in day $\left.^{-1}\right)$ was calculated by logging the medium (feed) and acid consumption over $24 \mathrm{~h}\left(\mathrm{~V}_{24 \mathrm{~h}}\right.$ in $\left.\mathrm{L}\right)$ and the volume of the photobioreactor ( $\mathrm{V}_{\mathrm{PBR}}$ in $\mathrm{L}$ ) (Eq. 4.1) (de Winter et al., 2017b):

$$
\mu_{24 h}=D_{24 h}=\frac{V_{24 h}}{V_{P B R}}
$$

Dilution rates over small intervals of time $\left(D_{t}\right)$ were calculated by logging the medium and acid consumption in intervals of $10 \mathrm{~min}$, followed by a moving average per $60 \mathrm{~min}$. Dilution patterns were repeated daily. Values corresponding to $1 \mathrm{~h}$ were averaged and used for the time-specific cell division rate $\left(\mu_{\mathrm{t}}\right)$, which was calculated based on a cell number balance ( $\left.C_{\text {cells }}\right)$ and $D_{t}$ following:

$$
\begin{aligned}
& \frac{d C_{\text {cells }}}{d_{t}}=-D_{t} \cdot C_{\text {Cells }}+\mu_{t} \cdot C_{\text {Cells }} \\
& \mu_{t}=\frac{\frac{d C_{\text {cells }}+D_{t} \cdot C_{\text {Cells }}}{d_{t}}}{C_{\text {Cells }}}
\end{aligned}
$$

Hourly values were added up to get the cumulative cell division rate. The average dilution rate over the light period was calculated by dividing the daily average dilution rate $\left(D_{24 h}\right)$ by the amount of hours of light supplied, with the following equation:

$$
D_{\text {over light period }}=\frac{D_{24 h} \cdot 24}{\text { hours of light supplied }}
$$

The doubling time $\left(t_{d}\right)$ was calculated as a function of the dilution rate $(D)$, using the following equation:

$$
t_{d}=\frac{\ln 2}{D}
$$

\subsubsection{Calculations}

Biomass yield on light (in $\mathrm{g}_{\mathrm{DW}} \cdot \mathrm{mol}_{\mathrm{ph}}{ }^{-1}$ ) was calculated as the ratio between the biomass productivity (in $\mathrm{g} \cdot \mathrm{L}^{-1} \cdot \mathrm{day}^{-1}$ ) and the photon absorption rate (in $\mathrm{mol}_{\mathrm{ph}} \mathrm{L}^{-1} \cdot \mathrm{day}^{-1}$ ). Starch productivity (in $\mathrm{g} \cdot \mathrm{L}^{-1} \cdot \mathrm{h}^{-1}$ ) was calculated using a balance for starch over short time intervals as explained by León-Saiki et al. (2017). The theoretical energy conversion 
efficiencies were calculated based on the theoretical photon requirements for the biomass components $\left(3.24 \mathrm{~g} \cdot \mathrm{mol}_{\mathrm{ph}^{-1}}\right.$ for starch and $1.62 \mathrm{~g} \cdot \mathrm{mol}_{\mathrm{ph}}{ }^{-1}$ for functional biomass (Kliphuis et al., 2012; Breuer et al., 2015a)) and the photon absorption rate (León-Saiki et al., 2017). Triacylglyceride (TAG) content remained below 1\% and was omitted for this calculation. Samples were taken in intervals of $3 \mathrm{~h}$ in the 14:10 $\mathrm{h} \mathrm{LD}$ and 12:12 h LD cycles. For the calculation of the hourly energy conversion efficiency in these 2 cycles, the additional points for biomass composition were estimated assuming a proportional change between the measured points.

\subsection{Results and discussion}

\subsubsection{Growth rate under different light regimes}

We started by characterizing growth of Tetradesmus obliquus wild-type and starchless mutant $\operatorname{sim} 1$ under light/dark (LD) cycles of 16:8 h, 14:10 h and 12:12 h. Since the reproducibility of the turbidostat set-up has been shown before (León-Saiki et al., 2017), one reactor run was done for each condition. The 16:8 h LD cultivation was repeated in this study and the results were comparable with our previous study for the wild-type and starchless mutant $\operatorname{s} / m 1$, as shown in Supplementary Figure S4.1. This shows the reproducibility of the experimental set-up.

During the light period, the cultures were diluted to maintain a constant light absorption over the culture and thus, a constant flux of photons to the culture inside the turbidostat controlled reactors. The dilution rate for the wild-type and s/m1 mutant under the three different LD cycles are presented in Figure 4.1. For the wild-type, maximum dilution rate was always reached 5-7 h after 'sunrise'. When comparing both strains, their dilution patterns were similar (Figure 4.1), where the wild-type reached higher dilution rates compared to the $/ \mathrm{m} 1$ mutant, indicating a faster growth rate. For the $s / m 1$ mutant, no difference in maximum dilution rate value was found between the photoperiods 14:10 $\mathrm{h}$ and 12:12 $\mathrm{h}$ LD $\left(0.07 \mathrm{~h}^{-1}\right)$ (Figure 4.1B). However, for the 16:8 $\mathrm{h}$ LD cycle, the maximum value reached was higher $\left(0.09 \mathrm{~h}^{-1}\right)$. Additionally, an unexplained oscillatory pattern was observed in this cycle for the mutant (Figure 4.1B), which was also observed in previous cultures at this LD cycle (Supplementary Figure S4.1). 
Table 4.1 shows for all conditions the average dilution rate over $24 \mathrm{~h}\left(\mathrm{D}_{24 h}\right)$, which is equal to the average growth rate. As can be seen, shorter light periods lead to lower dilution rates for both the wild-type and the $\operatorname{s} / m 1$ mutant, which is expected based on the fact that these cultures received a lower amount of light. Higher average $D_{24 h}$ translates into shorter doubling time $\left(t_{d}\right)$, as the microalgae are growing faster (Table 4.1). By looking into the doubling times (Table 4.1), it can be seen that for the wild-type this value is always below one per day, indicating that at least some cells must divide more than once per day. For the $\operatorname{s} \mid m 1$, doubling times are higher, specially under 14:10 $\mathrm{h}$ and 12:12 h LD cycles, indicating that cells divided approximately once per day. To verify if the difference in dilution rate can be fully explained by the difference in light received, the average dilution over the light period was calculated and is also shown in Table 4.1. This results in a more or less constant dilution rate for the $\operatorname{sim} 1$ mutant, while the wild-type actually shows an increase in the dilution with shorter day lengths. This means that the wild-type, during the time the light is on, grows faster at shorter light periods.
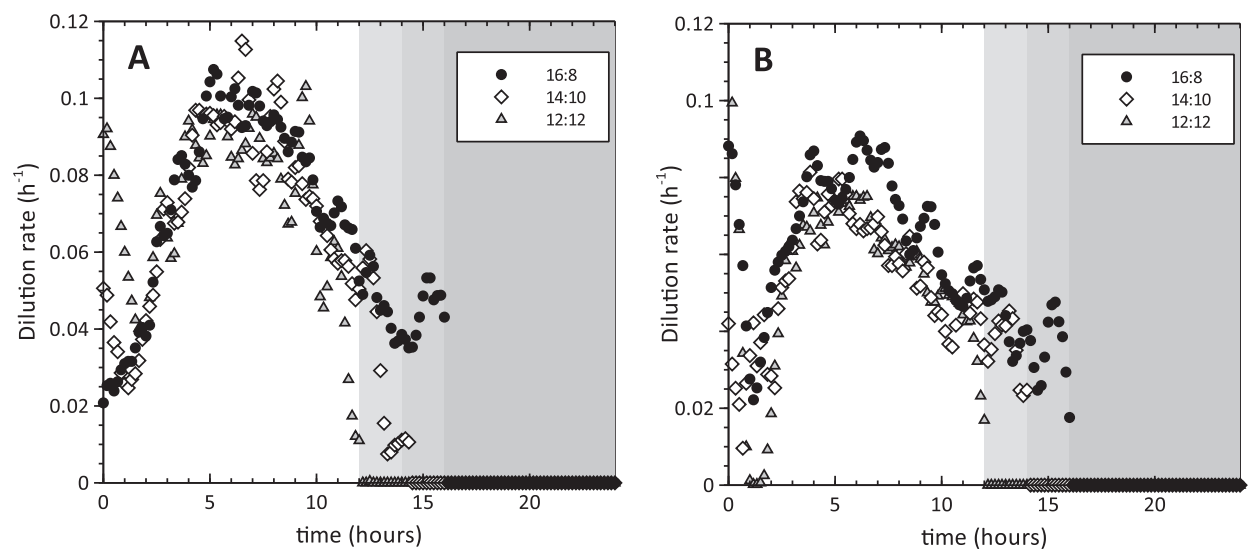

Figure 4.1. Changes in dilution rate over a $24 \mathrm{~h}$ period for Tetradesmus obliquus wild-type (A) and slm1 (B) during different photoperiods: 16:8 h light/dark (LD), 14:10 $\mathrm{h}$ LD and 12:12 h LD. The $x$ axis represents hours after 'sunrise'. Shaded area indicates the dark period. 
The light/dark periods at which microalgae are exposed impact biomass production and metabolism of microalgae (Jacob-Lopes et al., 2009; Krzemińska et al., 2014). As microalgal biomass production would be done outdoors with natural light/dark periods, the influence of LD cycles with different lengths has been studied. De Winter et al. (2017b) studied the influence of three different LD cycles: 20:4 h, 16:8 $\mathrm{h}$ and 12:12 h LD on growth of the microalga Neochloris oleoabundans under continuous turbidostat conditions. As expected, they also observed a decrease in the average $D_{24 h}$ with the shorter light periods. Dilution patterns were comparable with the ones observed for $T$. obliquus, reaching the maximum value approximately after $6 \mathrm{~h}$ of light and then decreasing until the end of the light period.

Table 4.1. Comparison of average dilution rate per $24 \mathrm{~h}\left(\mathrm{D}_{24 \mathrm{~h}}\right)$, doubling time and dilution per hour of light supplied of Tetradesmus obliquus wild-type and slm1 under 3 different light/dark cycles. Average $D_{24 h}$ values are shown as value \pm standard deviation for at least three daily values $(n \geq 3)$.

\begin{tabular}{lcccccccc}
\hline $\begin{array}{c}\text { Light/dark } \\
\text { period }\end{array}$ & \multicolumn{3}{c}{$\begin{array}{c}\text { Average } \mathrm{D}_{24 \mathrm{~h}} \\
\text { (day }{ }^{-1} \text { ) }\end{array}$} & \multicolumn{2}{c}{$\begin{array}{c}\text { Doubling time } \\
\text { (day) }\end{array}$} & \multicolumn{2}{c}{$\begin{array}{c}\text { Average D over } \\
\text { light period (day }{ }^{-1} \text { ) }\end{array}$} \\
& Wild-type & slm1 & Wild-type & slm1 & Wild-type & s/m1 \\
\hline $16: 8$ & 1.03 & \pm 0.05 & 0.93 & \pm 0.04 & 0.67 & 0.75 & 1.55 & 1.40 \\
$14: 10$ & 0.94 & \pm 0.06 & 0.76 & \pm 0.02 & 0.74 & 0.91 & 1.61 & 1.30 \\
$12: 12$ & 0.87 & \pm 0.02 & 0.66 & \pm 0.03 & 0.80 & 1.05 & 1.74 & 1.32 \\
\hline
\end{tabular}

Additionally, Jacob-Lopes et al. (2009) studied the influence of 12 different LD periods, from continuous light $(24: 0 \mathrm{~h})$ to $2: 22 \mathrm{~h}$ LD, on biomass production of the cyanobacterium Aphanothece microscopica Nägeli. They observed a linear reduction in biomass productivity with the reduction in the length of the light period, except for the $12: 12 \mathrm{~h} L D$, where the value did not follow the trend and increased compared to the value at 14:10 h LD cycle. Krzeminska et al. (2014) investigated the influence of light/dark cycles (12:12 h LD), compared to continuous light, on 5 different microalgae species: Neochloris conjuncta, Neochloris terrestris, Neochloris texensis, Botryococcus braunii and Tetradesmus obliquus under batch cultivation. They looked into the biomass doubling time and found that the microalgae $B$. braunii and $T$. obliquus had a higher growth rate under continuous light, while the three species of Neochloris grew better under 12:12 h LD cycles. The results obtained for T. obliquus agree with our results where the doubling time obtained under $12: 12 \mathrm{~h}$ LD (0.80 day) was higher than 
that obtained by León-Saiki et al. (2017) under continuous light (0.46 day). However, our values of doubling time were lower than those reported by Krzeminska et al. (2014) (1.17 \pm 0.02 day under $12: 12 \mathrm{~h}$ LD and $0.93 \pm 0.01$ day under continuous light), which could be related to the experimental conditions (such as cultivation medium and light settings).

Growth behavior of microalgal starchless mutants varies under nitrogen replete conditions. However, most of the studies have been carried out under continuous light. The starchless mutant BAFJ5 of Chlamydomonas reinhardtii showed reduced growth compared to its wild-type under continuous light (Li et al., 2010b), as was also observed for the starchless mutant of T. obliquus $\operatorname{s} / m 1$ under continuous light (León-Saiki et al., 2017). While Vonlanthen et al. (2015) found no significant difference in growth for the starchless mutant ST68 of Chlorella sorokiniana compared to the wild-type. For starchless mutants only one study could be found that was done under LD cycles. The growth of a starchless mutant of Chlorella pyrenoidosa STL-PI was studied under continuous light and a 12:12 h LD cycle (Ramazanov and Ramazanov, 2006). The authors found a higher growth rate of the mutant compared to the wild-type for both conditions, opposite to what we found for T. obliquus. This could be due to differences between these two species. Another explanation could be the fact that they based their conclusions on cell numbers and not on dry weight and that the amount of dry weight per cell is lower for the mutant than for the wild-type. However, this hypothesis cannot be tested as the results on dry weight production are not presented.

\subsubsection{Cell division}

Next we looked into the differences in cell division between the strains during the different LD cycles. First, we measured cell density in intervals of $1 \mathrm{~h}$ (Figure 4.2). Cell counts from León-Saiki et al. (2017) were included together with the data obtained by repeating the 16:8 $\mathrm{h}$ LD cycle. All cycles showed a decrease in cell number when the light started $(t=0)$ for a period of about $12 \mathrm{~h}$ due to dilution of the reactor. Cell numbers then stayed constant for a period of $3 \mathrm{~h}$ after which they increased sharply from $t=15 \mathrm{~h}$ to $\mathrm{t}=20 \mathrm{~h}$. Cell numbers differed between the photoperiods and strains. For the wild-type under 16:8 h LD, cell density went from approximately 29.8 million cells $\cdot \mathrm{mL}^{-1}$ to 61.4 million cells $\cdot \mathrm{mL}^{-1}$ (Figure $4.2 \mathrm{~A}$ ). For the $14: 10 \mathrm{~h} \mathrm{LD}$, cell density 
increased from 35.5 million cells $\cdot \mathrm{mL}^{-1}$ to 72.7 million cells $\mathrm{mL}^{-1}$. Finally, for the $12: 12 \mathrm{~h}$ LD cell density was lower and increased from 28.4 million cells $\cdot \mathrm{mL}^{-1}$ to 55.2 million cells $\cdot \mathrm{mL}^{-1}$. The higher cell density for the 14:10 h LD could be explained by the higher proportions in cells with lower diameter compared to the other two LD cycles (Supplementary Figure S4.2A). This indicates a lower absorbance per cell possibly due to a lower pigment content. For the wild-type, in general, cell number doubled within a small time-frame between 16-19 $\mathrm{h}$ for all the tested LD cycles.

The cell densities for the $\operatorname{s} / m 1$ were lower than for the wild-type in all tested LD cycles (Figure 4.2B). For the $16: 8 \mathrm{~h}$ LD, cell density went from 26.1 million cells $\cdot \mathrm{mL}^{-1}$ to 51.0 million cells $\cdot \mathrm{mL}^{-1}$. For the $14: 10 \mathrm{~h} \mathrm{LD}$, cell density increased from 26.8 million cells $\cdot \mathrm{mL}^{-1}$ to 39.4 million cells $\mathrm{mL}^{-1}$. Finally, for the $12: 12 \mathrm{~h} \mathrm{LD}$ cell density was lower and increased from 24.6 million cells $\cdot \mathrm{mL}^{-1}$ to 43.8 million cells $\cdot \mathrm{mL}^{-1}$. While for the wildtype the increase in cell density happened within a short time frame between 15-19 h, for the mutant under 16:8 h LD and 12:12 h LD cycles the increase started earlier and was slower (between 14-21 h). For the 14:10 h LD, cell increase was faster, taking only $2 \mathrm{~h}(\mathrm{t}=16 \mathrm{~h})$.
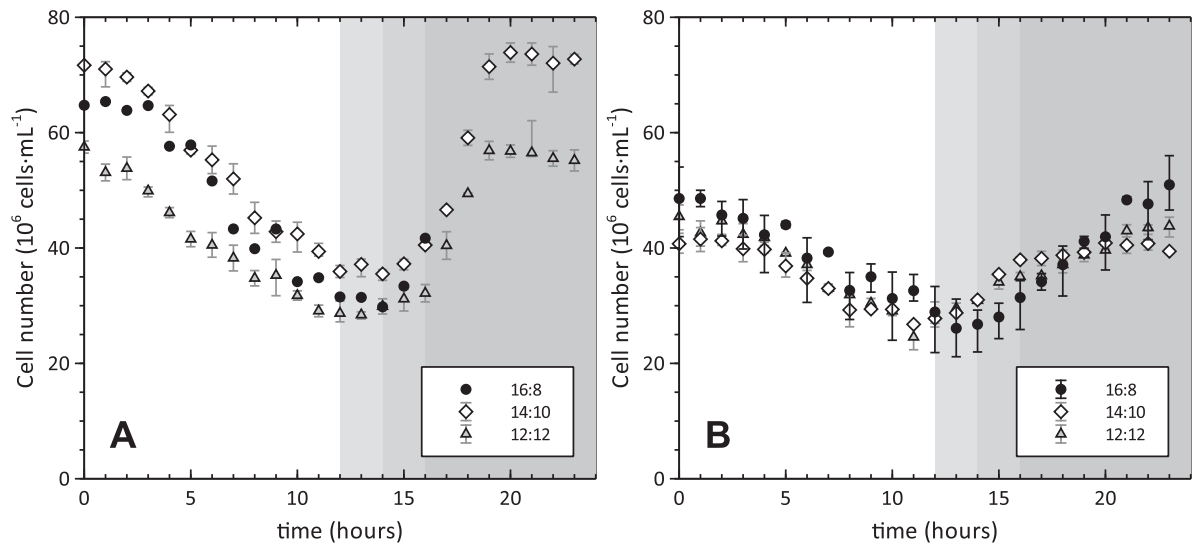

Figure 4.2. Cell density over a $24 \mathrm{~h}$ period for Tetradesmus obliquus wild-type (A) and $\operatorname{sim} 1$ (B). The $x$ axis represents hours after 'sunrise'. Shaded areas indicate the dark periods. Error bars show the highest and lowest values found for cell counts (with 2 or more data points used, $n \geq 2$ ), except for the wild-type under 16:8 h LD where some measurements were single and no error bars are shown. Data from León-Saiki et al. (2017) is included for the 16:8 h LD period. 
Since the change in cell numbers are a combined effect of cell division and dilution, we calculated the cell division rate to identify more precisely when cell division took place. For this the cell number data and the average dilution rate per hour were used, as explained in material and methods section 4.2.5. Figure 4.3 shows the cumulative average cell division rate for a certain time (t). This is the average proliferation per day over the period from $\mathrm{t}=0$ to $\mathrm{t}=\mathrm{t}$.

For the wild-type, there is a slow cell division rate during most of the day period, as can be seen from the small slope of the curve (Figure 4.3A). In general, cell division started around 14-15 $\mathrm{h}$ after the light went on, independently of the LD cycle, which is in accordance with the literature (de Winter et al., 2017b). Cell division stops 17-18 h after the light went on. For the 16:8 h LD cycle the sudden increase in cell division and the stop of cell division seem to start $1 \mathrm{~h}$ earlier than for the other two LD cycles. As expected, the final cumulative growth rate reached was comparable to the $24 \mathrm{~h}$ average dilution rate $\left(D_{24 h}\right)$ mentioned in Table 4.1 and was higher for longer light periods, which is directly related to the longer light periods.
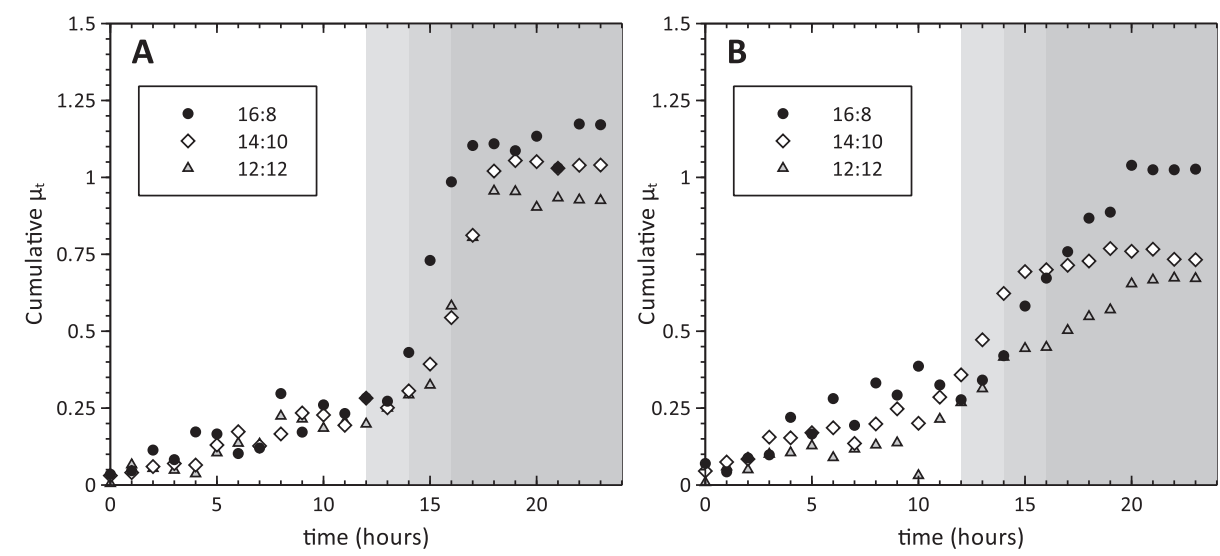

Figure 4.3. Cumulative average cell division rate $\left(\mu_{\mathrm{t}}\right)$ for Tetradesmus obliquus wildtype (A) and $s / m 1$ (B) during different photoperiods: 16:8 h LD, 14:10 $\mathrm{h}$ LD and 12:12 $\mathrm{h}$ LD. The $x$ axis represents hours after 'sunrise'. Shaded area indicates the dark period. 
Timing and patterns of cell division were different in the $\operatorname{s} \mid m 1$ compared to the wildtype. The slow cell division rate during the day is comparable to the wild-type. The sudden increase in cell division rate is less sharp for the $\operatorname{s} / m 1$ and seems to start earlier, around 10-12 $\mathrm{h}$ after 'sunrise' (14-15 h for the wild-type) independent of the length of the LD cycle applied. The period to complete cell division after the sudden increase at $10-12 \mathrm{~h}$ is longer for the mutant $(8 \mathrm{~h})$ than for the wild-type $(3 \mathrm{~h})$ (Figure 4.3B). When comparing the average cell size at the point where the sudden increase started (this is 14-15 $\mathrm{h}$ for the wild-type and 10-12 $\mathrm{h}$ for the $s / \mathrm{m} 1$ ), we observed that the average diameter is the same for both strains (Supplementary Figure S4.2C and D). This size could be an indicator of reaching the point after which cell division can occur independently of the presence of light (Oldenhof et al., 2007). The decreased cell division rate of the $s / m 1$, as well as the timing of the cell division (starting during the light period) is possibly related to the absence of a temporary energy storage compound (starch), which translates into less availability of carbon and energy.

As previously mentioned, light is one of the major cues for synchronization to daily cycles. Timing of cell division in microalgae has been suggested to depend on dawn (sunrise) and dusk (sunset) (de Winter et al., 2017b). For T. obliquus, sunrise seems to be the factor that defines timing of cell division, since the starting of cell division was similar (approximately $14 \mathrm{~h}$ for the wild-type and 10-12 $\mathrm{h}$ for the $\operatorname{sim} 1$ ), independently of the beginning of the dark period.

\subsubsection{Changes in starch content}

Concomitant with the synchronization to the LD cycles, cell composition changes throughout a 24 h cycle (Fábregas et al., 2002; Chauton et al., 2013; de Winter et al., 2013; Poliner et al., 2015). As previously observed, starch is the main component that shows oscillations in T. obliquus wild-type during light/dark cycles (León-Saiki et al., 2017).

As expected, starch was accumulated during the light period, and reached its maximum measured content when the night started (Figure 4.4A). The longer the light period, the higher the content of starch $\left(0.22 \mathrm{~g} \cdot \mathrm{gDW}^{-1}\right.$ for the $16: 8 \mathrm{~h} \mathrm{LD}, 0.18 \mathrm{~g} \cdot \mathrm{gDW}^{-1}$ for the 14:10 $\mathrm{h}$ LD and $0.16 \mathrm{~g} \cdot \mathrm{gDW}^{-1}$ for the 12:2 $\mathrm{h}$ LD cycle). In all LD cycles, starch content started increasing approximately $7 \mathrm{~h}$ after 'sunrise' (Figure 4.4A). A similar behavior 
was observed for the microalga Neochloris oleoabundans (de Winter et al., 2017b), where starch content started to increase 6-7 h after 'sunrise', with a higher maximum starch content with longer photoperiods.

To study the changes in starch production/consumption, we calculated the starch productivity during the measured time points (Figure 4.4B). Starch production rate started and increased about 5-7 h after the light went on. As soon as the dark period started, starch was consumed. As the light went on again starch consumption continued until $4 \mathrm{~h}$ after 'sunrise'. After this time point starch was depleted for the 16:8 $\mathrm{h}$ LD and 14:10 h. A notable exception was the 12:12 h LD showing no consumption in these $4 \mathrm{~h}$; also, the starch content did not become zero. It is unclear why $T$. obliquus did not totally consume the stored starch under this LD cycle, however, it should be mentioned that starch content was measured in intervals of $3 \mathrm{~h}$, and the point where starch is completely used as well as starch consumption may have been missed at this low resolution of measurements. Interestingly, the moment when starch is depleted coincides with the maximum value of the dilution rate (Figure $4.1 \mathrm{~A}$ ). At this maximum dilution rate, starch consumption switches to starch production (around $8 \mathrm{~h}$ after 'sunrise').
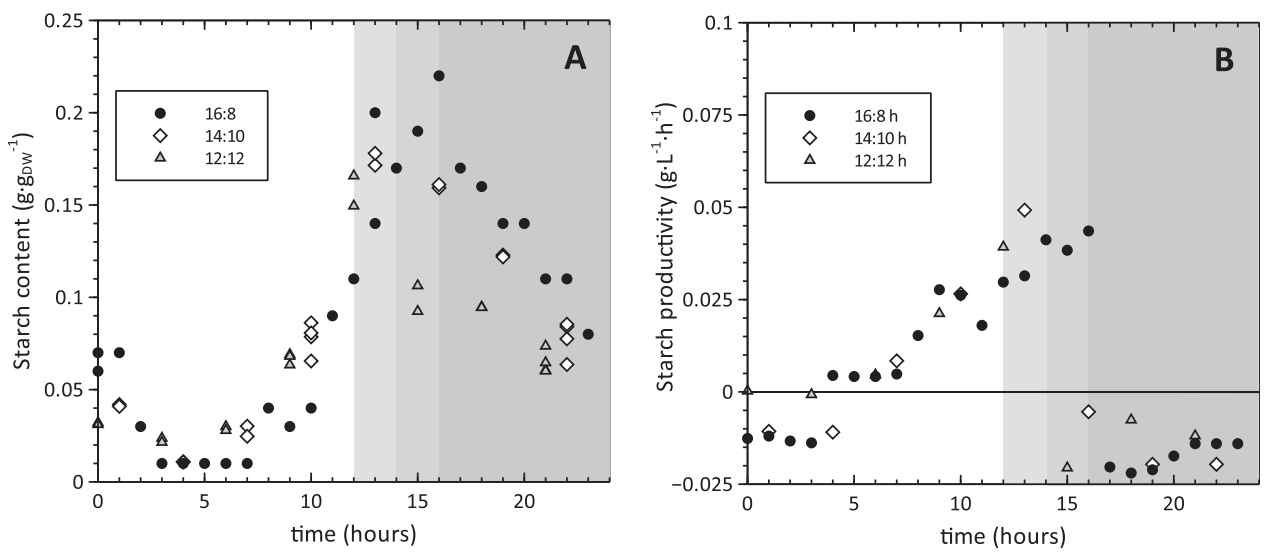

Figure 4.4. Changes in starch content $(A)$ and starch productivity (B) for Tetradesmus obliquus wild-type under different light/dark (LD) cycles. The $x$ axis represents hours after 'sunrise'. Shaded area indicates the different dark periods. Data for the 16:8 $\mathrm{h}$ LD cycle was provided by León-Saiki et al. (2017). 
The non-starch carbohydrates showed no changes through the measured points (Supplementary Figure S4.3). The protein content for the wild-type showed a decrease during the light period, which was concomitant with the increase in starch content (Supplementary Figure S4.3). For both strains, TAG content remained below $0.01 \mathrm{~g} \cdot \mathrm{gDw}^{-1}$ in all sampling points (not shown). For the starchless mutant s/m1, starch levels remained below $0.016 \mathrm{~g} \cdot \mathrm{g}_{\mathrm{DW}}{ }^{-1}$ during all sampled points in all photoperiods tested (not shown) and no fluctuations in biomass composition were observed for the tested LD cycles (Supplementary Figure S4.3).

\subsubsection{Efficiency of energy fixation in biomass components}

To calculate the energy conversion efficiency, i.e. the fraction of photons absorbed whose energy is fixed in biomass components, we measured the biomass composition (functional biomass and storage compounds, i.e. starch) of the daily overflows for both strains. The overflow composition remained similar during the different LD cycles (Table 4.2), but the average dry weight concentration, as well as the biomass productivity showed an increase with longer light periods. The TAG fraction was low (below $0.01 \mathrm{~g} \cdot \mathrm{g}_{\mathrm{DW}}{ }^{-1}$ ) and therefore not considered for the calculations. For T. obliquus wild-type a similar fraction of the supplied energy was fixed in biomass under 14:10 h $(54.61 \pm 0.07 \%)$ and $12: 12 \mathrm{~h}(55.18 \pm 0.06 \%)$ LD cycles (Figure 4.5A). These values were lower compared to the $16: 8 \mathrm{~h}$ LD cycle $(62.77 \pm 0.08 \%)$. This could be related to timing of cell division. The 16:8 h LD cycle is the only one where cell division starts during the day. Thus part of the energy needed for cell division may still come from the light, resulting in less use of starch during the night and allow to fix extra energy as compared to $14: 10 \mathrm{~h}$ and 12:12 h LD, where cell division occurs at night and relies completely on starch.

For the wild-type, the biomass yield on light was the highest with the longest light period (16:8 h LD) (Table 4.2). However, the increase does not continue until the dark period is skipped (continuous light), as the biomass yield on light was $0.98 \pm 00 \mathrm{~g} \cdot \mathrm{mol}_{\mathrm{ph}}{ }^{-1}$ (León-Saiki et al., 2017).

When the starch synthesis path is blocked, as in the $s / m 1$ mutant, the energy efficiency is lower than for the wild-type for all LD cycles (Figure 4.5A). However, the behavior was different compared to its wild-type. For the mutant, the highest biomass yield on 
light and the energy conversion efficiency occurred under 16:8 h LD cycle. This was surprisingly maintained during the 14:10 h LD, but dropped for the longest dark period tested (12:12 h LD cycle) (Table 4.2). This could also be related to timing of cell division. The 12:12 h LD cycle is the only tested photoperiod where cell division occurs mainly in the dark. This agrees with the slower division rates observed for this cycle compared to $16: 8 \mathrm{~h}$ and $14: 10 \mathrm{~h}$ LD where cell division starts during the day (Figure 4.3).

Table 4.2. Average steady state values for Tetradesmus obliquus wild-type and slm1 under different light/dark cycles. Average values are shown as value \pm standard deviations.

\begin{tabular}{|c|c|c|c|c|c|c|}
\hline & \multicolumn{3}{|c|}{ wild-type } & \multicolumn{3}{|c|}{$\operatorname{s} / m 1$} \\
\hline & $16: 8$ & $14: 10$ & $12: 12$ & $16: 8$ & $14: 10$ & $12: 12$ \\
\hline Dry weight concentration & 1.38 & 1.15 & 1.07 & 1.18 & 1.23 & 1.07 \\
\hline$\left(g \cdot L^{-1}\right)$ & \pm 0.07 & \pm 0.07 & \pm 0.04 & \pm 0.05 & \pm 0.03 & \pm 0.02 \\
\hline Biomass productivity & 1.42 & 1.08 & 0.93 & 1.10 & 0.94 & 0.70 \\
\hline$\left(\mathrm{g} \cdot \mathrm{L}^{-1} \cdot \mathrm{day}^{-1}\right)$ & \pm 0.08 & \pm 0.07 & \pm 0.04 & \pm 0.07 & \pm 0.03 & \pm 0.03 \\
\hline Biomass yield on light & 1.04 & 0.91 & 0.91 & 0.81 & 0.79 & 0.68 \\
\hline$\left(\mathrm{g} \cdot \mathrm{mol}_{\mathrm{ph}}{ }^{-1}\right)$ & \pm 0.08 & \pm 0.09 & \pm 0.04 & \pm 0.07 & \pm 0.04 & \pm 0.03 \\
\hline Starch & $0.05^{*}$ & 0.05 & 0.03 & $0^{*}$ & 0 & 0 \\
\hline$\left(g \cdot g_{D w}{ }^{-1}\right)$ & \pm 0.01 & \pm 0.01 & \pm 0.01 & & & \\
\hline $\begin{array}{l}\text { Photon absorption rate } \\
\left(\mathrm{mol}_{\mathrm{ph}} \cdot \mathrm{L}^{-1} \cdot \mathrm{day}^{-1}\right)\end{array}$ & 1.36 & 1.19 & 1.02 & 1.36 & 1.19 & 1.02 \\
\hline
\end{tabular}

Standard deviations were calculated based on at least 3 overflows. Except for the wildtype under $12: 12 \mathrm{~h} \mathrm{LD}$ where $\mathrm{n}=2$, and the value represents the average $\pm \mathrm{max} / \mathrm{min}$ values.

During a LD cycle the dilution rate and biomass composition change. Since the photon absorption rate is constant, the energy conversion efficiency will change over a LD cycle. To get more insight into this, the energy efficiency was calculated in one hour intervals based on the measured biomass composition, dilution rate and photon absorption rate at each time point. By looking at the energy conversion efficiency during the daily cycles, we found that the highest energy fixation occurs with the maximum dilution rate (Figure $4.5 \mathrm{~B}$ and $\mathrm{C}$ ). Both strains showed similar patterns but, as expected, the wild-type reached higher values (approximately $90 \%$ ) compared to the starchless mutant $\operatorname{s} / m 1$ (maximum $80 \%$ ). This maximum value decreased slightly with the shorter light periods for both the mutant and wild-type. 

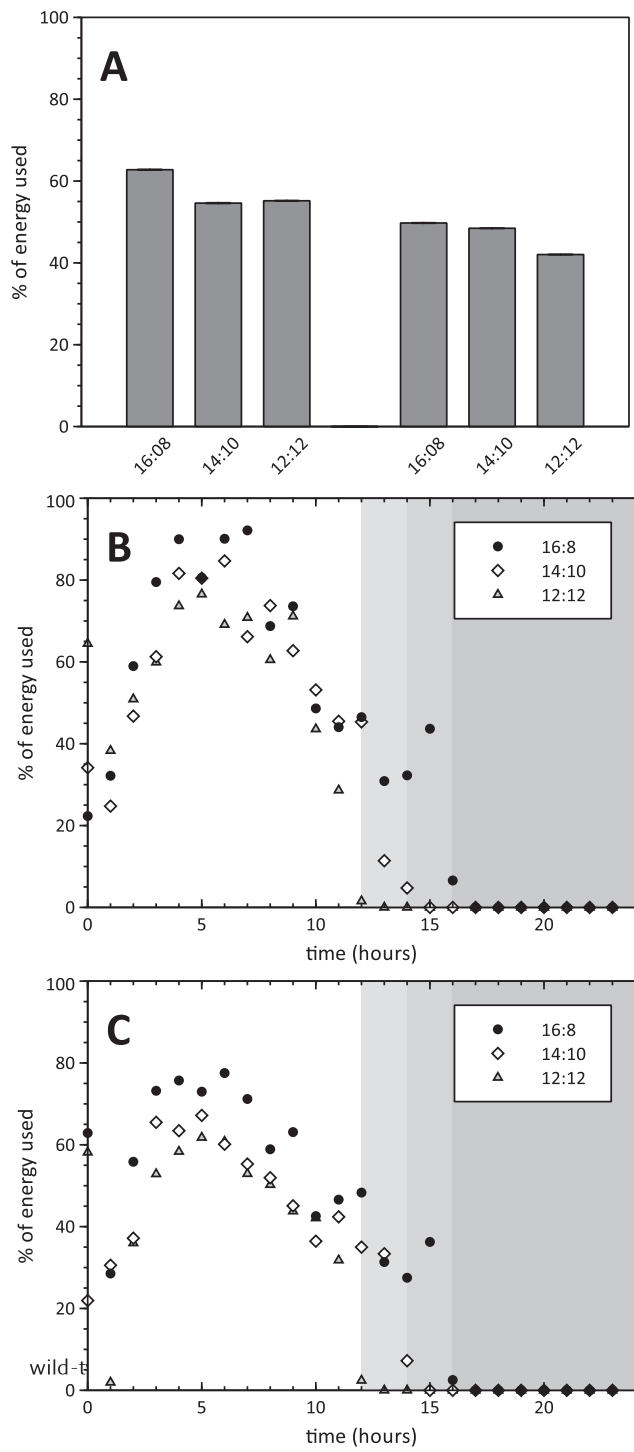

Figure 4.5. Theoretical photon efficiency as percentage of photons used to make biomass components (A) and hourly percentage of photons used for Tetradesmus obliquus wild-type (B) and slm1 (C) under different light/dark (LD) cycles. Values represent averages of at least 3 daily overflows for (A). Error bars represent the standard deviations calculated by error propagation. For the hourly energy conversion efficiency the $x$ axis represents hours after 'sunrise'. Shaded area indicates the dark period. Data for the 16:8 h LD cycle was provided by León-Saiki et al. (2017). 
At the start of the day starch is still used. Probably this is for the rapid synthesis of absorbing material to harvest light energy, which can be deduced from the rapid increase in dilution rate. Depletion of the starch coincides with the maximum energy efficiency and dilution rate. From that moment on starch is net synthesized and the starch content increases again. During the night, starch is used again for cell division and preparation for the moment when light goes on again. This can be derived from the fact that cell division is much slower in the mutant and the fact that for the mutant the increase in dilution rate starts later after the light is turned on. Apparently, starch is used during the night to prepare for the next light cycle or during the night energy is derived from cell components that have to be built up again if the light goes on.

\subsection{Conclusions}

The start of the light phase (sunrise) is the reference point for synchronized cell division in Tetradesmus obliquus. Cell division in T. obliquus wild-type started approximately $14 \mathrm{~h}$ after sunrise, independently of the length of the light period. Cell division occurred mainly at night, except during the longest light period tested (16:8 h light/dark cycle), where cell division started at the end of the light period and extended into the dark period. For the s/m1 mutant, cell division was also synchronized to the start of the light period but started earlier than for the wild-type (10-12 $\mathrm{h}$ after sunrise). Regarding biomass composition, the length of the day had an influence on the maximum starch content reached by the wild-type: the longer the light period, the higher the starch content. The starchless mutant $\operatorname{s} / m 1$ showed no oscillations in biomass composition. But, despite the lack of starch, the s/m1 mutant synchronized growth and cell division to the LD cycles. However, the absence of starch resulted in lower energy efficiencies (11-24\% lower) and biomass yield on light (13-39\% lower) for T. obliquus s/m1 compared to its wild-type under all tested LD cycles.

\section{Funding}

This research project was supported by the Consejo Nacional de Ciencia y Tecnología - CONACYT, Mexico, Scholar 218586/Scholarship 314173. In addition, GMLS is part of the program "Doctores Jóvenes para el Desarrollo Estratégico Institucional" by the Universidad Autónoma de Sinaloa. 


\section{Supplementary information}
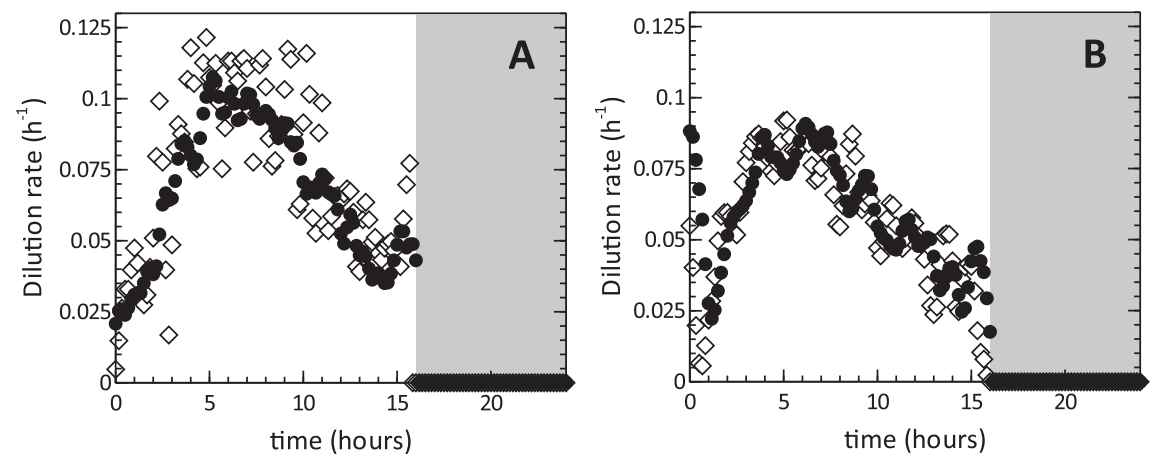

Supplementary Figure S4.1. Comparison of dilution rate patterns for the $16: 8 \mathrm{~h}$ light/dark cycles for Tetradesmus obliquus wild-type (A) and slm1 (B). Black circles represent results from this paper, grey diamonds from León-Saiki et al. (2017).
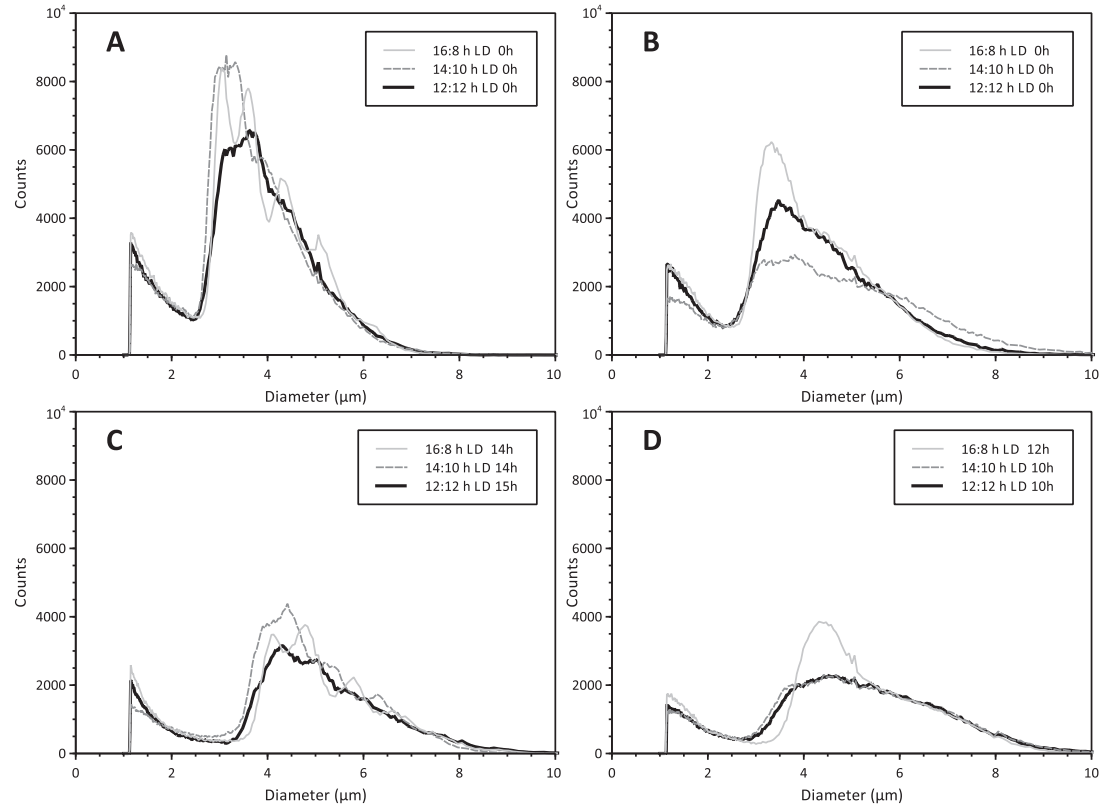

Supplementary Figure S4.2. Comparison of cell diameter for the wild-type (left) and slm1 (right) during the tested photoperiods. Values correspond to the beginning of the light period $(t=0)(A$ and $B)$ and before the beginning of sharp increase in cell division rate ( $C$ and $D$ ) (times indicated in the key box). 

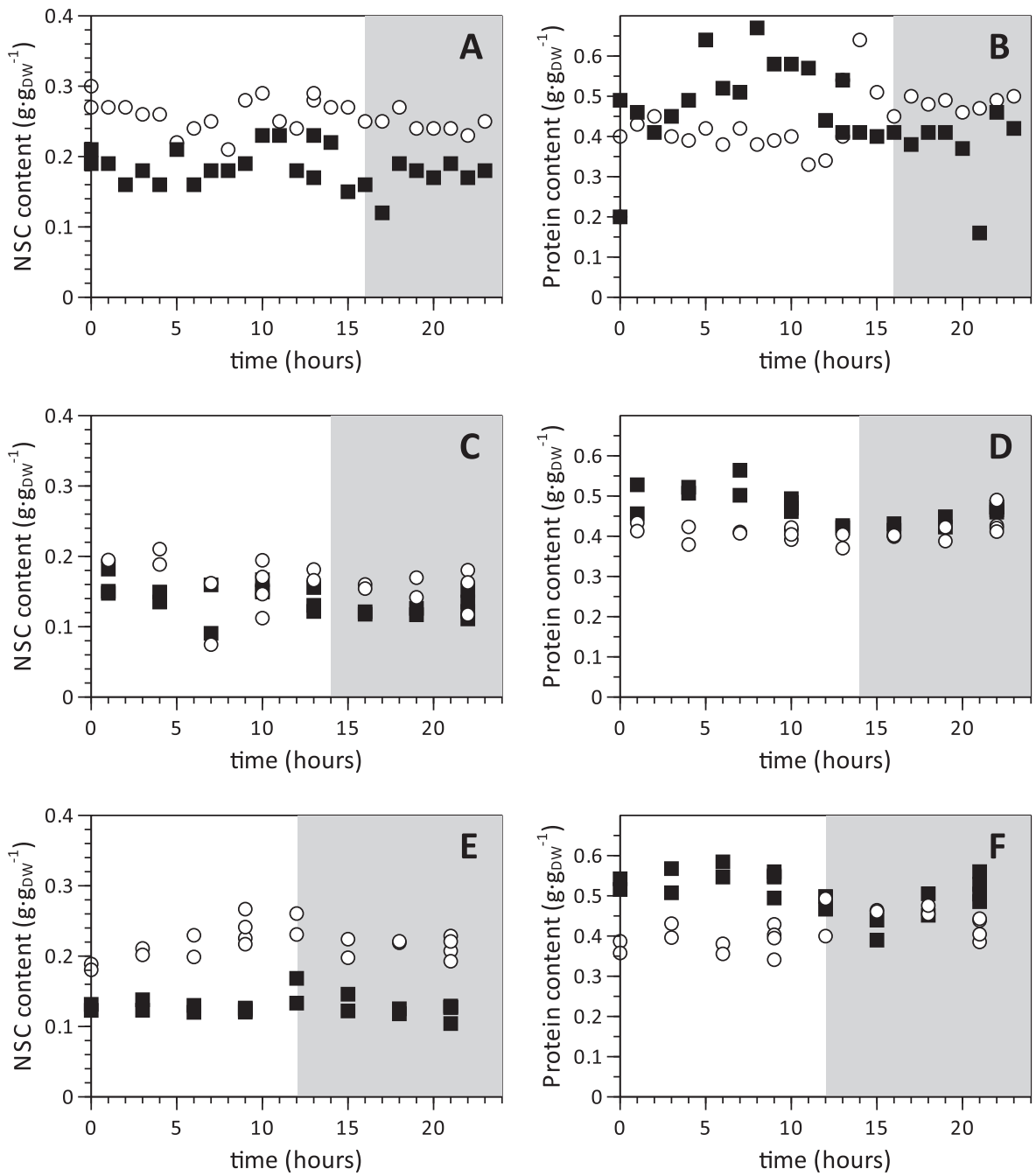

Supplementary Figure S4.3. Changes in non-starch carbohydrates and protein content for Tetradesmus obliquus wild-type and $s / m 1$ under different light/dark (LD) cycles. Non-starch carbohydrates (NSC): 16:8 h LD (A), 14:10 h LD (C) and 12:12 h LD (E). Protein: 16:8 h LD (B), 14:10 h LD (D) and 12:12 h LD (F). Closed squares represent the wild-type and opened circles represent the $\operatorname{sim1} 1$. The $\mathrm{x}$ axis represents hours after 'sunrise'. Shaded area indicates the dark period. Data for the 16:8 h LD cycle was provided by León-Saiki et al. (2017). 


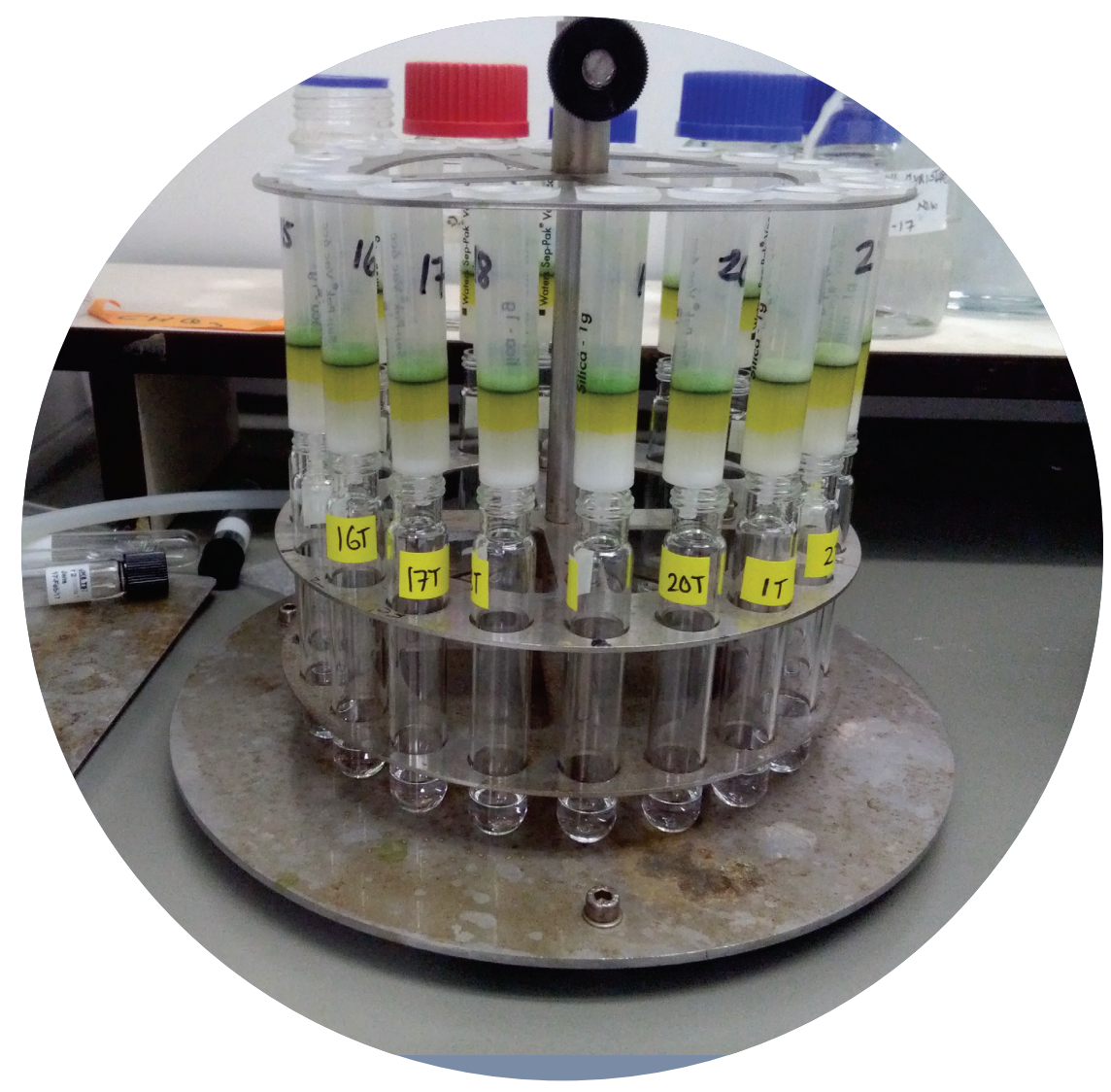




\section{Chapter}

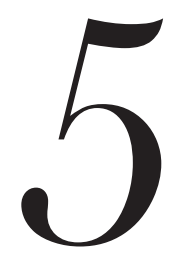

\section{Diurnal biochemical responses of Tetradesmus obliquus under light/dark cycles and nitrogen limitation}


In this work the diurnal behaviour during light/dark cycles and nitrogen limitation of Tetradesmus obliquus wild-type and starchless mutant $\operatorname{sim} 1$ is studied. During nitrogen limitation, the wild-type continued to use starch as the preferred storage compound to store energy and carbon. Starch was accumulated to an average content of $0.25 \mathrm{~g} \cdot \mathrm{gDW}^{-1}$, which is higher than the maximum observed under nitrogen replete conditions. Small oscillations were observed, indicating that starch was being used as a diurnal energy storage compound, but to a lesser extent than under nitrogen replete conditions. For the s/m1 mutant, TAG content was higher than for the wildtype (steady state value from the overflow was $0.23 \mathrm{~g} \cdot \mathrm{gDw}^{-1}$ for $\mathrm{s} / \mathrm{m} 1$ compared to $0.07 \mathrm{~g} \cdot \mathrm{g}_{\mathrm{DW}}{ }^{-1}$ for the wild-type). However despite the higher TAG content in the $\operatorname{sim} 1$, the conversion efficiency of photons into biomass components for the $\operatorname{sim} 1$ was only half of the one obtained for the wild-type. This is related to the observed decrease in biomass productivity (from 1.29 $\mathrm{g}_{\mathrm{DW}} \cdot \mathrm{L}^{-1} \cdot \mathrm{day}^{-1}$ for the wild-type compared to $0.52 \mathrm{~g}_{\mathrm{DW}} \cdot \mathrm{L}^{-1} \cdot \mathrm{day}^{-1}$ for the $\operatorname{s} / \mathrm{m} 1$ ). When looking through the diurnal cycle, the photosynthetic efficiency was lower for the $\operatorname{s} / m 1$ mutant compared to the wild-type, especially during the second half of the light period, where starch accumulation occurred in the wild-type. 


\subsection{Introduction}

Microalgae are considered as one of the most promising renewable feedstocks for the production of feed, fuels and chemicals (Wijffels and Barbosa, 2010; Chisti, 2013; Klok et al., 2014). However, in order to make large scale production of algal biodiesel economically feasible, high triacylglycerides (TAG) productivities are needed (Wijffels and Barbosa, 2010; Klok et al., 2013).

Tetradesmus obliquus (formerly known as Scenedesmus obliquus (Krienitz and Bock, 2012)) is a microalga of interest due to its high TAG content and the fact that it can retain a high photosynthetic efficiency under nitrogen starvation (Mandal and Mallick, 2009; Ho et al., 2012; Breuer et al., 2013b; Remmers et al., 2017). Besides, de Jaeger et al. (2014) developed the starchless mutant $\operatorname{sim} 1$ that showed a higher maximum TAG yield on light compared to the wild-type $\left(0.22 \mathrm{~g}_{\mathrm{TAG}} \cdot \mathrm{mol}_{\mathrm{ph}}{ }^{-1}\right.$ compared to $\left.0.14 \mathrm{~g}_{\mathrm{TAG}} \cdot \mathrm{mol}_{\mathrm{ph}}{ }^{-1}\right)$, as well as a higher maximum TAG content $\left(0.57 \mathrm{~g} \cdot \mathrm{g}_{\mathrm{DW}}{ }^{-1}\right.$ compared to $0.45 \mathrm{~g} \cdot \mathrm{gDW}^{-1}$ ) under batch nitrogen starvation and continuous illumination (Breuer et al., 2014).

In large scale production, microalgal biomass will be grown outdoors under favourable nitrogen replete conditions and light/dark cycles (LD). After biomass has been produced, the lipid production phase can be done under nitrogen deplete conditions. The behaviour of T. obliquus under nitrogen replete conditions and diurnal 16:8 h light/dark (LD) cycles was previously studied (León-Saiki et al., 2017). Under such conditions, $T$. obliquus wild-type and starchless mutant $s / m 1$ showed synchronized cell division and growth. T. obliquus wild-type showed diurnal oscillations in biomass composition, with an accumulation of starch during the light period that was consumed during the dark period and beginning of light period. For the $\operatorname{s} / m 1$, no such oscillations in biomass composition were found, which shows that this microalga does not need a temporary energy storage compound to survive dark periods to up to $12 \mathrm{~h}$ (León-Saiki et al., 2018). The lack of starch, however, resulted in a reduction of the conversion of energy (photons) to biomass (León-Saiki et al., 2017; León-Saiki et al., 2018).

Under nitrogen deplete conditions, many microalgal species accumulate starch and/or TAG, which allow the capture of energy and carbon that can be rapidly used when nitrogen becomes available again (Mulders et al., 2014). Since production of TAG 
occurs under nitrogen limitation or starvation and LD cycles, the behaviour of T. obliquus under these conditions is of interest. The overall steady state behaviour under nitrogen limitation and LD cycles of both T. obliquus wild-type and $s / m 1$ has been previously reported (Remmers et al., 2017). However, the detailed diurnal behaviour during a single LD cycle under nitrogen limitation has not been studied in these strains. Therefore, the aim of this paper is to obtain a better understanding of the diurnal behaviour of $T$. obliquus wild-type and starchless mutant $s / m 1$ under nitrogen limitation, with focus on the diurnal changes in starch and TAG content, and on energy efficiency.

\subsection{Materials and Methods}

\subsubsection{Strains, pre-culture conditions and cultivation medium}

Wild-type Tetradesmus obliquus UTEX 393 was obtained from the Culture Collection of Algae, University of Texas. The starchless mutant of $T$. obliquus (s/m1) was generated by de Jaeger et al. (2014). Pre-cultures were grown as described by León-Saiki et al. (2017) in defined medium described by Breuer et al. (2013b).

\subsubsection{Rector set-up and experimental conditions}

T. obliquus was continuously cultivated in a sterilised flat panel airlift-loop photobioreactor with a working volume of $1.7 \mathrm{~L}$ and a $0.02 \mathrm{~m}$ light path (Labfors $5 \mathrm{Lux}$, Infors HT, Switzerland). Temperature was maintained at $27.5^{\circ} \mathrm{C}$ and $\mathrm{pH}$ was controlled at 7.0 by the automatic addition of $2.5 \%(\mathrm{v} / \mathrm{v}) \mathrm{H}_{2} \mathrm{SO}_{4}$. The reactor was continuously sparged with $1 \mathrm{~L} \cdot \mathrm{min}^{-1}$ air enriched with $2 \% \mathrm{CO}_{2}$. Light was provided by a light panel with 260 LEDs with a warm white spectrum at an incident photon flux density of $500 \mu \mathrm{mol} \cdot \mathrm{m}^{-2} \cdot \mathrm{s}^{-1}$ in a $16: 8 \mathrm{~h}$ light/dark (LD) block cycle. The reactor was inoculated to an optical density $\left(O D_{750}\right)$ of 0.1 .

Duplicate turbidostat cultivations were done, where the light intensity at the rear of the reactor was kept constant at $10 \mu \mathrm{mol} \cdot \mathrm{m}^{-2} \cdot \mathrm{s}^{-1}$ by addition of medium. Dilution medium (Breuer et al., 2013b) was prepared without $\mathrm{KNO}_{3}$ and $\mathrm{KNO}_{3}$ was separately fed at $0.075 \mathrm{gN}_{\mathrm{N}} \cdot \mathrm{L}^{-1} \mathrm{day}^{-1}$ for the wild-type and $0.052 \mathrm{gN}_{\mathrm{N}} \mathrm{L}^{-1} \mathrm{day}^{-1}$ for the $\operatorname{s} / \mathrm{m} 1$. This corresponds to a nitrogen limitation of $30 \%$ of the nitrogen consumption rate observed 
under nitrogen replete and light limitation (Remmers et al., 2017). Dilution medium was only added during the light period and switched off during the night. This type of system allows the study of the interaction between growth, nitrogen consumption and lipid accumulation (Klok et al., 2013).

Cultures were allowed to reach steady state, which was defined as a constant biomass concentration and daily dilution rate for a period of at least 3 days. After steady state was reached, liquid samples were freshly taken from the reactor and either immediately used for dry weight measurements or centrifuged for $5 \mathrm{~min}$ at $2360 \mathrm{xg}$ for biochemical analysis (approximately 10-12 mg for proteins, $5 \mathrm{mg}$ for starch, $7 \mathrm{mg}$ for triacylglycerides (TAG) and $5 \mathrm{mg}$ for total carbohydrates). In addition, at least three daily overflow samples were collected for each strain.

\subsubsection{Dilution rate}

The dilution rate was calculated by logging the feed of the dilution medium (without $\mathrm{KNO}_{3}$ ) and the feed of the $\mathrm{KNO}_{3}$ medium in intervals of $10 \mathrm{~min}$. Dilution rates were then calculated as the total medium added over $30 \mathrm{~min}$ divided by the reactor volume. After that, a moving average of $60 \mathrm{~min}$ was done.

\subsubsection{Analyses}

Dry weight (DW) concentration was determined as described by Kliphuis et al. (2012). Total carbohydrates were determined according to DuBois et al. (1956) and Hebert et al. (1971) using glucose as a standard. Starch content was measured as described by de Jaeger et al. (2014) using a total starch kit (Megazyme, Ireland). Triacylglycerol (TAG) content was quantified as described by Remmers et al. (2017) using glyceryl trinonadecanoate (T4632; Sigma Aldrich) and 1,2-dipentadecanoyl-sn-glycero-3phospho-(1'-rac-glycerol) (sodium salt) (840446, Avanti Polar Lipids Inc.) as internal standards. Protein analysis as done using a colorimetric assay (Bio-Rad DC protein assay) as described by Postma et al. (2015). 


\subsubsection{Conversion of photons into biomass}

The theoretical amount of photons converted into biomass was calculated based on the theoretical photon requirements for the biomass components $\left(1.33 \mathrm{~g} \cdot \mathrm{mol}_{\mathrm{ph}}{ }^{-1}\right.$ for TAG, $3.24 \mathrm{~g} \cdot \mathrm{molph}^{-1}$ for starch, and $1.62 \mathrm{~g} \cdot \mathrm{molph}^{-1}$ for functional biomass (Kliphuis et al., 2012; Breuer et al., 2015a)) and the photon absorption rate (León-Saiki et al., 2017). Samples were taken in intervals of three hours. For the calculation of the hourly energy conversion efficiency the additional points for biomass composition were estimated assuming a proportional change between the measured points. Biomass composition of the overflow samples (collected during $24 \mathrm{~h}$ on ice) was used to calculate the average steady state values.

\subsubsection{Starch and TAG productivity}

The starch productivity $\left(r_{\text {starch, }}\right.$ in $\left.\mathrm{g} \cdot \mathrm{L}^{-1} \cdot \mathrm{h}^{-1}\right)$ was calculated using a balance for starch over short time intervals (Eq. 5.1), considering the change in starch content over three hour time intervals $\left(\frac{d C_{s t a r c h}}{d t}\right)$, the dilution rate $\left(D_{t}\right.$ in $\left.h^{-1}\right)$ and the starch content $\left(C_{\text {starch, } t}\right.$ in $\left.g \cdot L^{-1}\right):$

$$
\frac{d C_{\text {starch }}}{d t}=-D_{t} \cdot C_{\text {starch }, t}+r_{\text {starch }, t}
$$

The TAG productivity was calculated in a similar way where the changes in TAG content over intervals of 3 hours $\left(\frac{d C_{T A G}}{d t}\right)$, the dilution rate $\left(D_{t}\right.$ in $\left.h^{-1}\right)$, and the TAG content $\left(C_{T A G, t}\right.$ in $\mathrm{g} \cdot \mathrm{L}^{-1}$ ) were used.

\subsection{Results and discussion}

\subsubsection{Growth of Tetradesmus obliquus wild-type and slm1}

Our experimental set-up using turbidostat controlled systems imposes a fixed light uptake. We created an energy imbalance by decreasing the nitrogen supply rate to $30 \%$ of the value that would be needed to have nitrogen replete growth at the fixed light uptake rate. 
Cells responded to this limitation by reducing their growth rate (Figure 5.1). Nitrogen limited cultures showed a diurnal pattern in dilution rate, with both the wild-type and the starchless mutant $\operatorname{s} / m 1$ showing a similar pattern. Dilution patterns were, however, different than those under nitrogen replete conditions, specifically the start of dilution after sunrise and the moment where the maximum dilution rate was reached. Dilution started about $4 \mathrm{~h}$ after 'sunrise' under nitrogen limitation, while under nitrogen replete conditions dilution started immediately after the beginning of the light period. As a result, the time at which the maximum dilution rate was reached was also shifted (approximately $4 \mathrm{~h}$ after sunrise under nitrogen replete compared to $7 \mathrm{~h}$ after 'sunrise' for nitrogen limitation). A possible explanation for this shift may be that, as nitrogen was not added during the night, nitrogen content was equal to zero (starvation). This may have resulted in cells being unable to prepare for the next day, or cells switching to a nitrogen starvation mode from which they had to recover. With respect to the first case, it is possible that cells prepare during the night for the next day so they can start growing quickly. If nitrogen is needed for this preparation, cells would have to first fixate nitrogen when the light is on, which would explain the delay. With respect to the second case, cells switch to a nitrogen starvation mode, breaking down pigments or stopping pigment synthesis, which would result in a decreased pigment content at the beginning of the light period as supported by the fact that the light out for the wildtype was $1 \mu \mathrm{mol} \cdot \mathrm{m}^{-2} \cdot \mathrm{s}^{-1}$ above setpoint. Consequently, first a certain amount of pigment has to be synthesized before the light out drops below the setpoint and dilution starts. For the mutant an unexplained oscillatory pattern was observed (Figure 5.1B), which was also observed for this strain under nitrogen replete conditions and a 16:8 h LD cycle.

Under growth conditions (nitrogen replete), oleaginous microalgae produce only small amounts of TAG while under unfavourable nitrogen starvation conditions, TAG accumulation is induced and contents of up to $0.40 \mathrm{~g} \cdot \mathrm{gDW}^{-1}$ can be reached (Hu et al., 2008; Breuer et al., 2012). However, this unfavourable environmental conditions come with the disadvantage of a complete stop of cell division (Remmers et al., 2017). Nitrogen limitation processes were suggested as an attempt to overcome the disadvantages observed under nitrogen starvation (Klok et al., 2013). As observed for T. obliquus, growth still occurs under nitrogen limitation, but this is reduced to approximately half of the one observed under nitrogen replete conditions (from 
$1.12 \pm 0.01$ day $^{-1}$ under nitrogen replete (León-Saiki et al., 2017) to $0.55 \pm 0.07$ day $^{-1}$ under nitrogen limitation). This was also observed for the microalga Neochloris oleoabundans (de Winter et al., 2014). For the starchless mutant $\operatorname{s} / m 1$ the $24 \mathrm{~h}$ average daily dilution rate, i.e. growth rate, was reduced even more (from $0.90 \pm 0.01$ day $^{-1}$ under nitrogen replete to $0.22 \pm 0.02$ day $^{-1}$ under nitrogen limitation), showing again the reduction in growth and lower photosynthetic efficiency when blocking starch production.
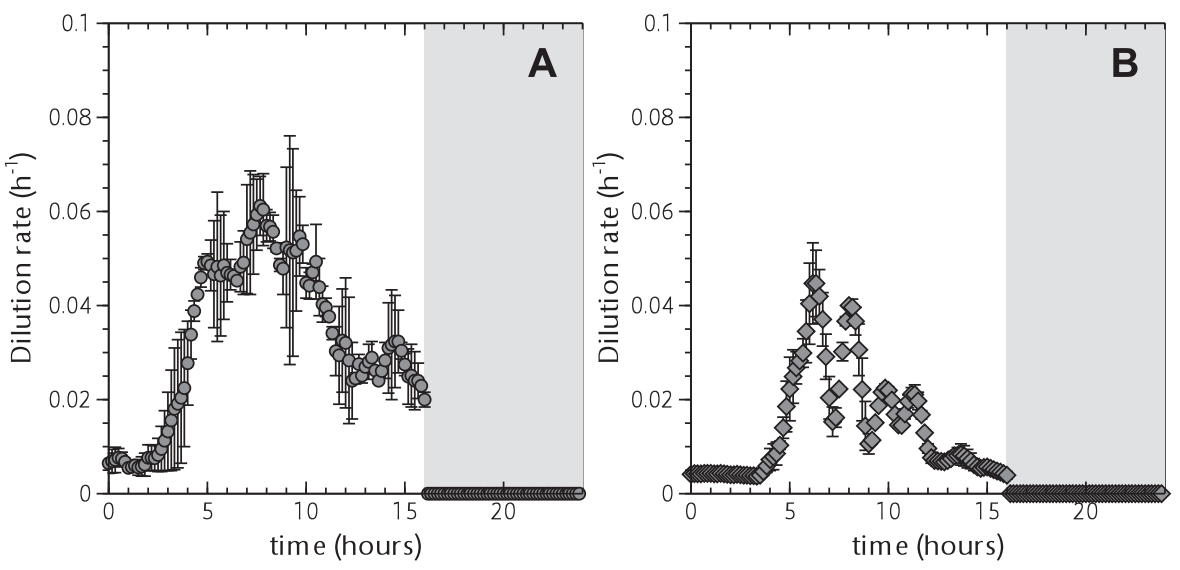

Figure 5.1. Changes in dilution rate for Tetradesmus obliquus wild-type (A) and slm1 (B) under nitrogen limitation. Values represent the average of replicate reactors and error bars represent minimum and maximum values. The $x$ axis shows hours after 'sunrise'. Shaded areas indicate the dark period.

\subsubsection{Diurnal changes of storage compounds: starch and TAG}

During nitrogen limitation, starch continued to be the preferred storage compound to store energy and carbon for the wild-type (Figure 5.2A). Starch was accumulated to an average content of $0.25 \mathrm{~g} \cdot \mathrm{gDw}_{\mathrm{DW}}{ }^{-1}$, with a maximum content of approximately $0.29 \mathrm{~g} \cdot \mathrm{gDw}^{-1}$, which is higher than the maximum observed under nitrogen replete conditions ( $0.20 \mathrm{~g} \cdot \mathrm{gDW}_{\mathrm{DW}}^{-1}$ ) (León-Saiki et al., 2017). The overall preference of $T$. obliquus wild-type towards storing starch has been previously observed (Breuer et al., 2014; Remmers et al., 2017). When looking into the starch content during the diurnal cycle, a small oscillation can be observed (Figure 5.2A), where starch slightly increases between 9-12 $\mathrm{h}$ and decreases during the dark period. This can also be seen in the 
starch productivity (Figure 5.2C), which remained approximately zero during the first $9 \mathrm{~h}$, then increased from $\mathrm{t}=12 \mathrm{~h}$ until $\mathrm{t}=21 \mathrm{~h}$ after which starch productivity was negative, indicating consumption.
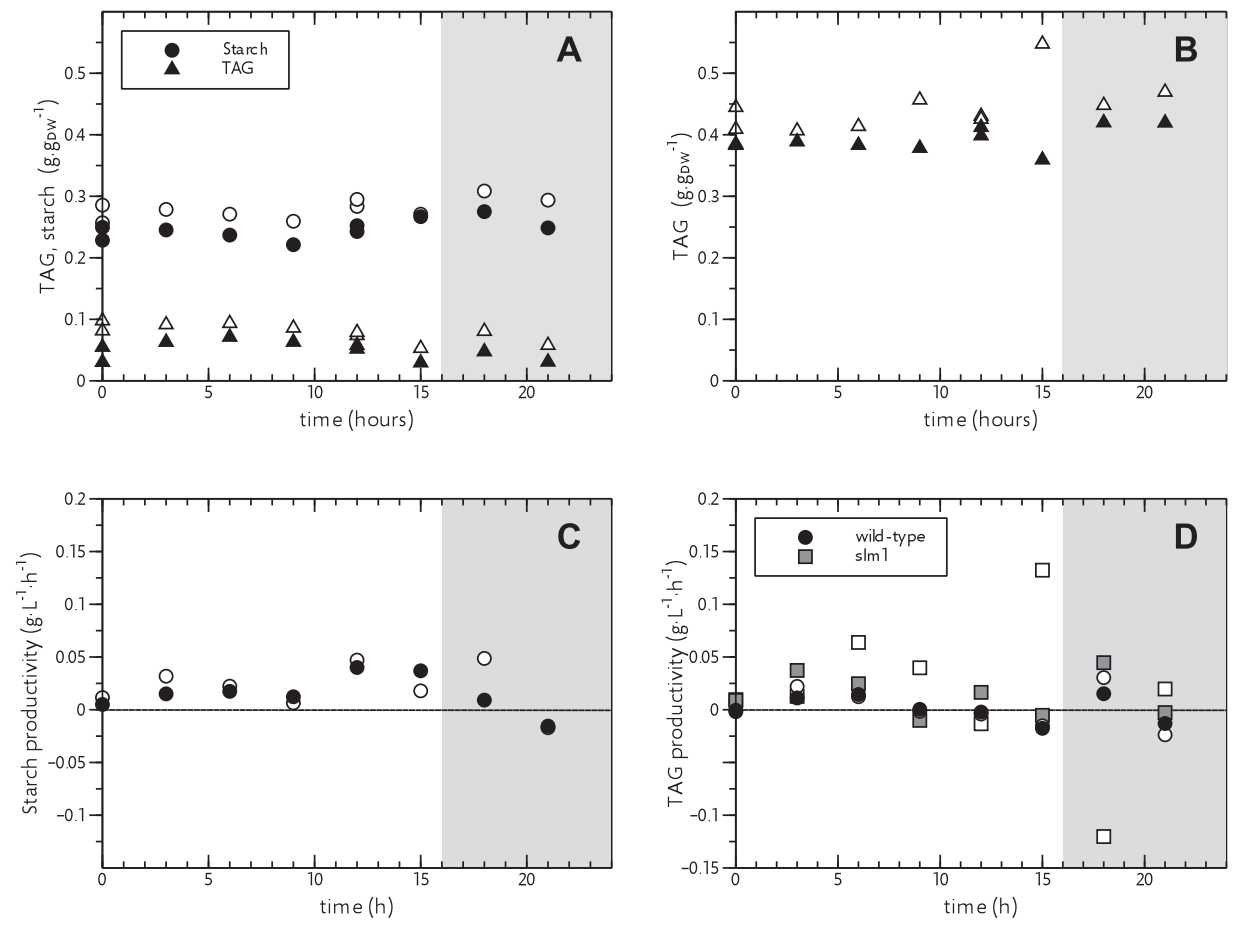

Figure 5.2. Diurnal changes under nitrogen limitation in starch and triacylglycerides (TAG) content for Tetradesmus obliquus wild-type (A) and slm1 (B) and in starch productivity for the wild-type (C) and TAG productivity for wild-type and $s / m 1$ (D). Open and closed symbols represent replicate cultures. The $x$ axis shows hours after 'sunrise'. Shaded areas indicate the dark period.

Under nitrogen replete conditions, TAG is not accumulated, whereas under nitrogen limitation or starvation substantial amounts of TAG can be accumulated in T. obliquus, as observed by Remmers et al. (2017). However, no information is known on the diurnal role of this compound under nitrogen limitation in T. obliquus. Therefore, we also measured the TAG content during the diurnal cycle in intervals of $3 \mathrm{~h}$. The wildtype showed a constant content of approximately $0.06 \mathrm{~g} \cdot \mathrm{g}_{\mathrm{DW}}{ }^{-1}$ for all measured time points (Figure 5.2A). This rather low content of TAG can be explained by the fact that carbon and energy are mainly stored as starch. TAG is only formed if the carbon and 
energy supply exceeds the storage capacity in starch, as also mentioned in literature (Fan et al., 2012; Breuer et al., 2014; Remmers et al., 2017). For T. obliquus, Remmers et al. (2017) found that TAG is only accumulated after starch reaches its cellular maximum of $0.40 \mathrm{~g} \cdot \mathrm{gDW}^{-1}$. Under this nitrogen limitation condition this maximum content is not reached, which explains the low content of TAG in the wild-type. The preference for starch cannot be explained from the energy density, since more energy can be stored in fatty acids as compared to starch (yield of complete oxidation of fatty acids is about $9 \mathrm{kcal} \cdot \mathrm{g}^{-1}$ compared to $4 \mathrm{kcal} \cdot \mathrm{g}^{-1}$ for carbohydrates (Berg et al., 2002)). A possible explanation for the preference for starch may be that the synthesis and degradation of fatty acids, components of TAG, are slower compared to carbohydrates (Lancelot and Mathot, 1985). Another reason might be that when cells grow photoautotrophically, fatty acids synthesis represent a significant loss of carbon. This is because the conversion of pyruvate (C3) to acetyl-CoA (C2) by the pyruvate dehydrogenase complex involves the loss of one of the fixed carbons. This means that, in terms of carbon fixation, starch is a more efficient storage compound for carbon (Johnson and Alric, 2013).

For the $\operatorname{sim} 1$ mutant the TAG content (approximately $0.42 \mathrm{~g} \cdot \mathrm{g}_{\mathrm{DW}}{ }^{-1}$ ) was about 7 times higher than for the wild-type during the diurnal cycle (Figure 5.2B). However, TAG showed no clear diurnal oscillations under nitrogen limitation for either strain (Figure 5.2A and B). When looking at the TAG productivity, diurnal variations were expected for the $\operatorname{sim} 1$ as the dilution rate is changing. However, no clear diurnal variations were observed (Figure 5.2D). This is because the values for dilution rate are similar or smaller than the accumulation term $\left(\frac{d C_{T A G}}{d t}\right)$. The accumulation term is in turn highly sensitive for small changes in the measured TAG content. This is, the TAG productivity for the slm1 is highly affected by measurement errors in the TAG content. For the wild-type, the values of the dilution rate are higher, making the calculation of the TAG productivity less sensitive to small errors in the TAG content, which in turn makes the differences between the time points more significant. 


\subsubsection{Energy conversion efficiency}

Under nitrogen replete conditions, the energy conversion efficiency into biomass of the wild-type is higher than that of the $\operatorname{s} / m 1$ mutant (León-Saiki et al., 2017; León-Saiki et al., 2018). We therefore investigated if this was also the case for nitrogen limitation conditions where TAG accumulation occurs. For this we calculated the minimal number of photons needed for the production of functional biomass, TAG, and starch (for the wild-type), based on the biomass composition of the overflow samples (average steady state values). Results are displayed as a percentage of used photons from the total amount supplied in Figure 5.3A. As can be seen, also for nitrogen limited conditions the efficiency is lower for the $\operatorname{s} / m 1$ mutant than for the wild-type (approximately $50 \%$ for the wild-type and $25 \%$ for the $\operatorname{sim} 1$ ). Even though the TAG content on the $\operatorname{sim} 1$ is higher than for the wild-type, this lower efficiency is related to the fact that the biomass productivity is reduced to less than half $\left(1.29 \pm 0.11 \mathrm{~g} \cdot \mathrm{L}^{-1} \cdot \mathrm{day}^{-1}\right.$ for the wild-type and $0.52 \pm 0.06 \mathrm{~g} \cdot \mathrm{L}^{-1} \cdot \mathrm{day}^{-1}$ ) (Supplementary Table S5.1). Also, both strains are less efficient under nitrogen limited conditions compared to nitrogen replete conditions ( $62.77 \pm 0.08 \%$ for the wild-type and $49.75 \pm 0.07 \%$ for the $\operatorname{s} / m 1$ (León-Saiki et al., 2018)).

When looking into the conversion efficiency during the diurnal cycle in intervals of one hour (Figure 5.3B and C), we observe that during the first half of the day, both strains behave similarly, with the wild-type showing an increase in energy use one hour before the $\operatorname{sim} 1$. However, in the second half of the day (approximately from $\mathrm{t}=7 \mathrm{~h}$ ), the $\operatorname{sim} 1$ mutant is much less efficient than the wild-type. Notably the second half of the light period is the moment when starch accumulation occurs in the wild-type, probably explaining the extra energy fixated.

Under nitrogen starvation, both starch and TAG can act as an overflow sink for electrons (Hu et al., 2008). For starchless mutants, it has been suggested that the difference in energy efficiency and biomass yield on light might be due to an increased rate of energy dissipation compared to the wild-type (Li et al., 2010b; Remmers et al., 2017) since electrons can no longer be channeled to starch. However, under nitrogen limitation growth still occurs and starch still seems to have a role as a diurnal energy storage compound, such as under nitrogen replete conditions (León-Saiki et al., 2017), which provides an extra benefit for the wild-type compared to the $\operatorname{sim} 1$. 

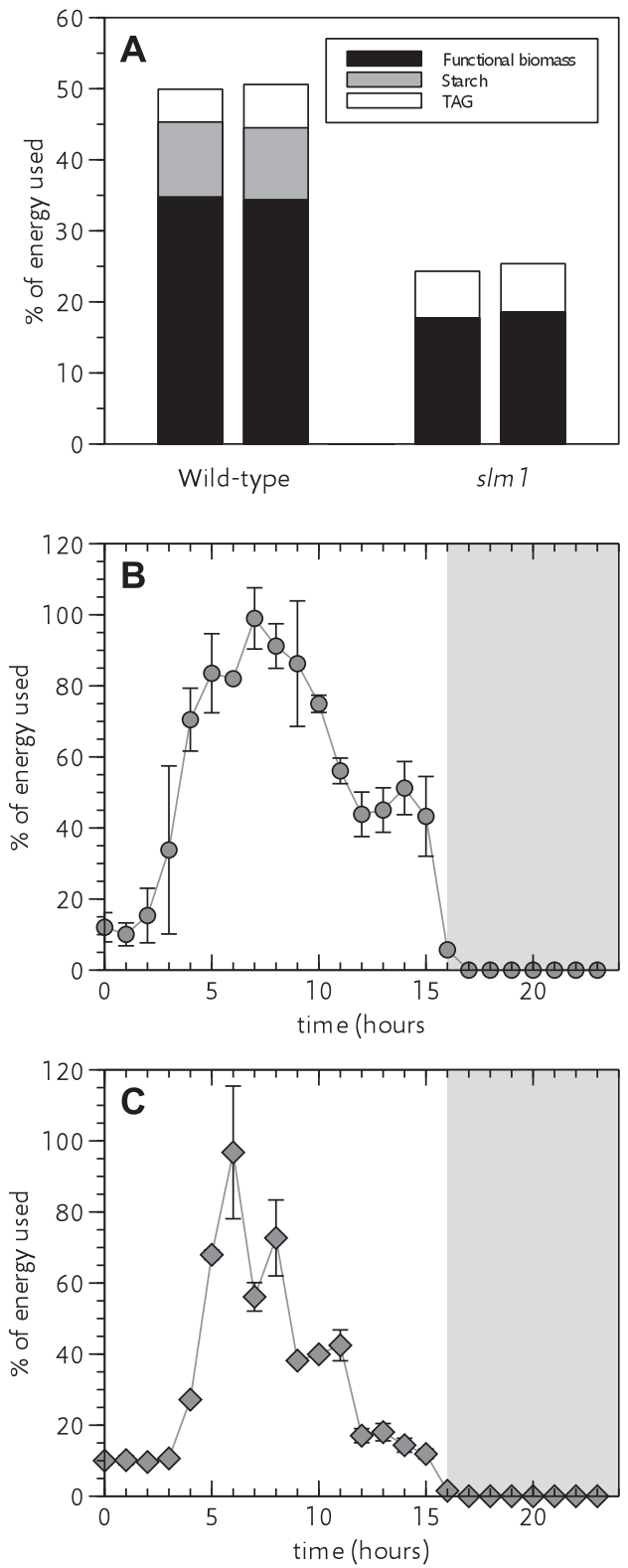

Figure 5.3. The percentage of photons (as a fraction of the total supplied) minimally needed to make the different biomass components (A) and hourly percentage of photons used by Tetradesmus obliquus wild-type (B) and $s / m 1$ (C). For (A), values represent the average values of at least 3 overflow samples. For the hourly energy conversion efficiency ( $B$ and $C$ ) the $x$ axis represents hours after 'sunrise'. Values represent average of replicate reactors and error bars represent minimum and maximum values. Shaded area indicates the dark period. 


\subsection{Conclusions}

Under continuous nitrogen limitation, both Tetradesmus obliquus wild-type and s/m1 showed a repeated diurnal dilution pattern, with the s/m1 showing a lower $24 \mathrm{~h}$ dilution rate (growth rate) compared to the wild-type. Under this conditions, T. obliquus wild-type continued to prefer starch to store energy and carbon, and starch was accumulated to an average content of approximately $0.25 \mathrm{~g} \cdot \mathrm{g}_{\mathrm{DW}}{ }^{-1}$. Additionally, starch showed small diurnal oscillations, where starch content increased during the last hours of the light period (10-16 h) and decreased during the dark period. This provided an advantage for the wild-type compared to the $\operatorname{sim1}$, as the energy conversion efficiency into the biomass components during the diurnal cycle was higher for the wild-type, especially during the last hours of light, where starch is accumulated. Even though the TAG content in the $\operatorname{s} / m 1$ was higher than for the wild-type (average steady state values of approximately $0.23 \mathrm{~g} \cdot \mathrm{gDW}^{-1}$ for the $\operatorname{sim} 1$ compared to $0.07 \mathrm{~g} \cdot \mathrm{gDW}^{-}$ ${ }^{1}$ for the wild-type), the conversion efficiency of photons into biomass components for the $\operatorname{sim} 1$ was only half of the one obtained for the wild-type, which is due to the decrease in biomass productivity in the $\operatorname{s} / m 1$ (from $1.29 \mathrm{gDw} \cdot \mathrm{L}^{-1} \cdot \mathrm{day}^{-1}$ for the wild-type to $0.52 \mathrm{gDW}_{\mathrm{DW}} \mathrm{L}^{-1} \cdot \mathrm{day}^{-1}$ for the $\left.\operatorname{s} / \mathrm{m} 1\right)$.

\section{Funding}

This research project was supported by the Consejo Nacional de Ciencia y Tecnología - CONACYT, Mexico, Scholar 218586/Scholarship 314173. In addition, GMLS is part of the program "Doctores Jóvenes para el Desarrollo Estratégico Institucional" by the Universidad Autónoma de Sinaloa. 


\section{Supplementary information}

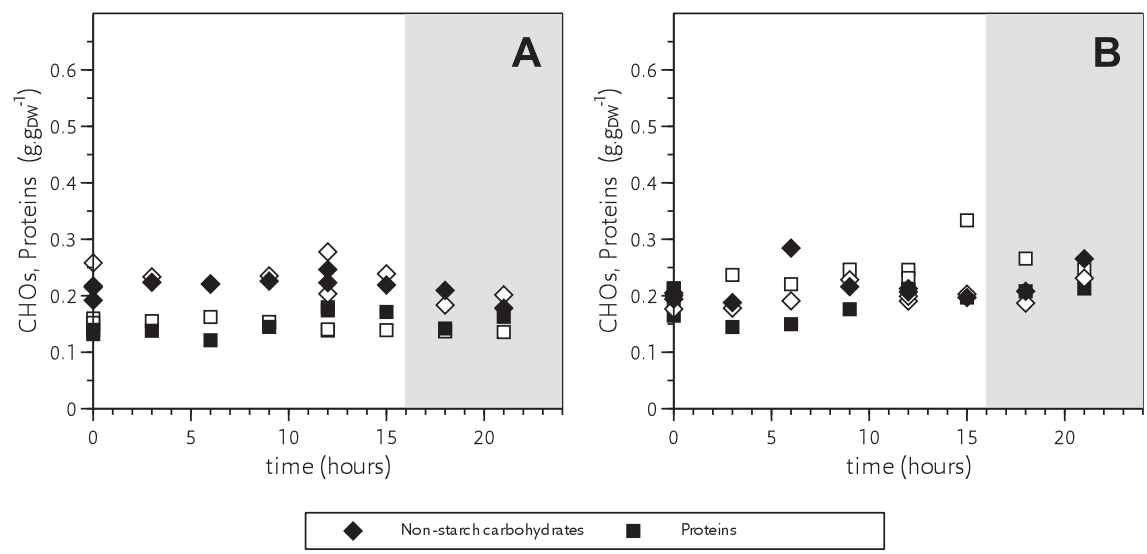

Supplementary Figure S5.1. Diurnal changes in non-starch carbohydrates (diamonds) and proteins (squares) for Tetradesmus obliquus wild-type (A) and slm1 (B) under nitrogen limitation. Open and closed symbols represent replicate cultures. The $x$ axis shows hours after 'sunrise'. Shaded areas indicate the dark period.

Supplementary Table S5.1. Average steady state values for Tetradesmus obliquus wild-type and $s / m 1$ under nitrogen limitation. Average values are given for biological duplicate reactor runs. Average values are shown as value \pm standard deviations.

\begin{tabular}{|c|c|c|c|c|}
\hline \multirow[b]{2}{*}{ Dry weight concentration $\left(\mathrm{g} \cdot \mathrm{L}^{-1}\right)$} & \multicolumn{2}{|c|}{ Wild-type } & \multicolumn{2}{|c|}{$s / m 1$} \\
\hline & $2.16 \pm 0.17$ & $2.55 \pm 0.18$ & $2.42 \pm 0.17$ & $2.40 \pm 0.10$ \\
\hline Dilution rate $\left(\right.$ day $\left.^{-1}\right)$ & $0.61 \pm 0.05$ & $0.50 \pm 0.04$ & $0.21 \pm 0.02$ & $0.22 \pm 0.02$ \\
\hline Biomass productivity $\left(\mathrm{g} \cdot \mathrm{L}^{-1} \cdot\right.$ day $\left.^{-1}\right)$ & $1.31 \pm 0.13$ & $1.27 \pm 0.11$ & $0.51 \pm 0.06$ & $0.53 \pm 0.07$ \\
\hline Biomass yield on light $\left(\mathrm{g} \cdot \mathrm{mol}_{\mathrm{ph}}{ }^{-1}\right)$ & $0.97 \pm 0.09$ & $0.93 \pm 0.08$ & $0.37 \pm 0.04$ & $0.39 \pm 0.05$ \\
\hline Triacylglycerides $\left(g \cdot g_{D W}{ }^{-1}\right)$ & $0.06 \pm 0.01$ & $0.08 \pm 0.01$ & $0.23 \pm 0.02$ & $0.23 \pm 0.01$ \\
\hline Starch $\left(g \cdot g_{D w^{-1}}\right)$ & $0.35 \pm 0.03$ & $0.34 \pm 0.02$ & - & - \\
\hline $\begin{array}{l}\text { Photon absorption rate } \\
\left(\mathrm{mol}_{\text {photons }} \cdot \mathrm{L}^{-1} \cdot \text { day }^{-1}\right)\end{array}$ & \multicolumn{2}{|c|}{1.36} & \multicolumn{2}{|c|}{1.36} \\
\hline
\end{tabular}




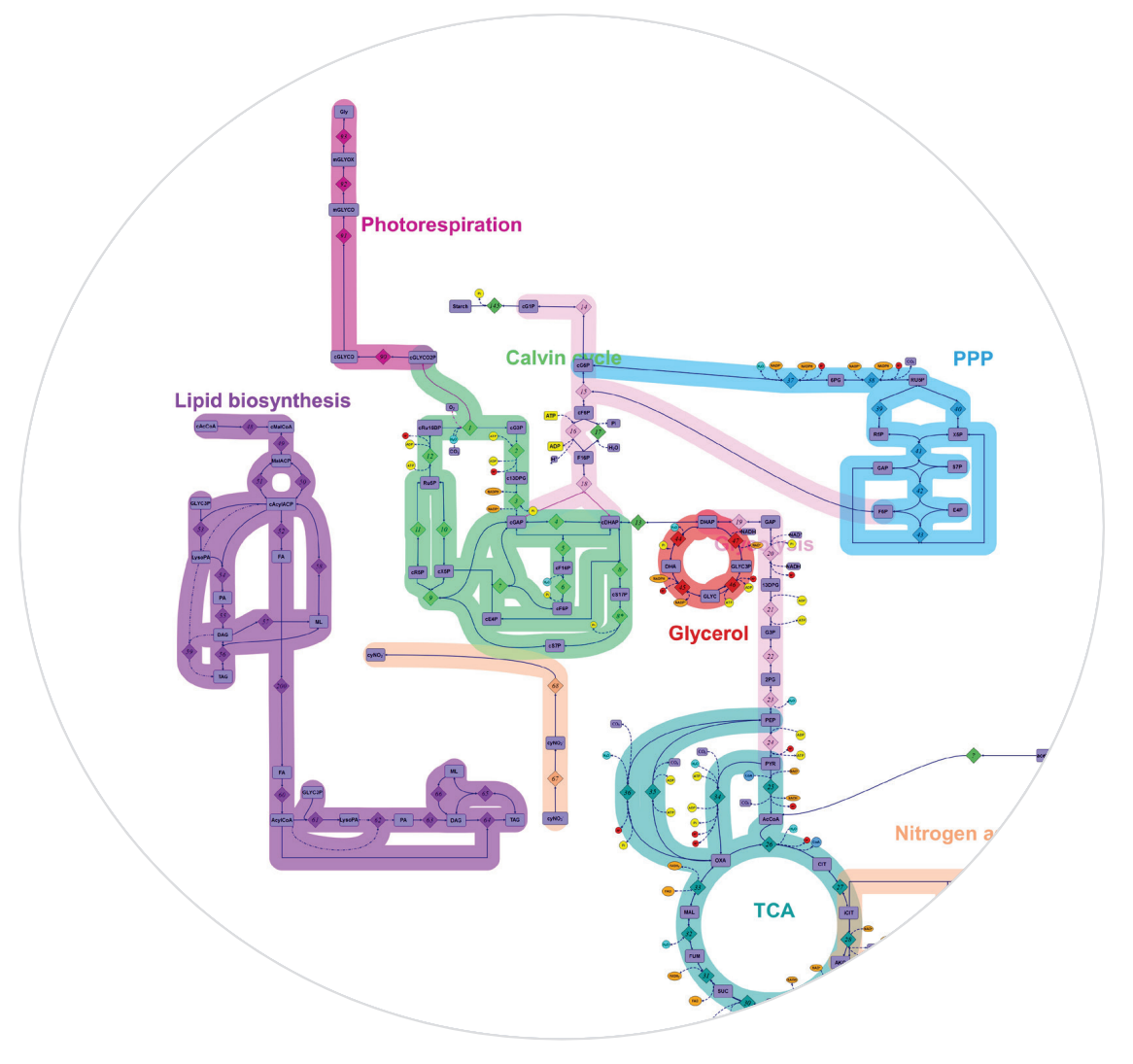




\section{Chapter}

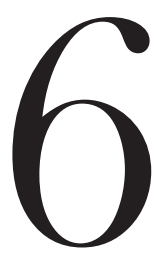

\section{Metabolic modelling and energy parameter estimation of Tetradesmus obliquus}

This chapter is submitted for publication as:

GM León-Saiki, N Ferrer Ledo, D Lao-Martil, D van der Veen, RH Wijffels, DE Martens Metabolic modelling and energy parameter estimation of Tetradesmus 
We developed a metabolic network describing the primary metabolism of Tetradesmus obliquus aimed to get a better understanding of metabolism to improve industrial production. The network includes 351 reactions with 183 metabolites distributed over 4 compartments: cytosol, chloroplast, mitochondria, and extracellular space. The energy requirements for biomass assembly and maintenance ( $K_{\mathrm{x}}$ and $\mathrm{m}_{\mathrm{ATP}}$, respectively) were experimentally determined from batch cultures and included in the model. The determined values were $121.02 \mathrm{mmol}_{\text {ATP }} \cdot \mathrm{gDW}^{-1}$ for $\mathrm{K}_{\mathrm{x}}$ and $0.66 \mathrm{mmol}_{\mathrm{ATP}} \cdot \mathrm{gDW}^{-1} \cdot \mathrm{h}^{-1}$ for the $\mathrm{m}_{\text {ATP. }}$. The maintenance value found for $T$. obliquus is, to our knowledge, one of the lowest reported in literature for microalgae. This low value is also in agreement with the photon maintenance requirement found experimentally for T. obliquus $\left(1.18 \mathrm{mmol}_{\mathrm{ph}} \cdot \mathrm{gDW}^{-1} \cdot \mathrm{h}^{-1}\right)$. Finally, the theoretical maximum yields based on the model for biomass, triacylglycerides (TAG), and starch yield on light were calculated to be $1.15 \mathrm{~g} \cdot \mathrm{mol}_{\mathrm{ph}}{ }^{-1}, 1.05 \mathrm{~g}_{\mathrm{TAG}} \cdot \mathrm{mol}_{\mathrm{ph}}{ }^{-1}$, and $2.69 \mathrm{~g}_{\mathrm{starch}} \cdot \mathrm{mol}_{\mathrm{ph}}{ }^{-1}$. 


\subsection{Introduction}

Microalgae are promising sustainable cell factories for the production of food, feed, nutraceuticals, chemicals, and fuels due to their photosynthetic ability and product diversity (Wijffels et al., 2013; Guarnieri and Pienkos, 2015). The oleaginous microalgae Tetradesmus obliquus (formerly known as Scenedesmus obliquus (Krienitz and Bock, 2012)) is an industrially relevant strain that can accumulate high amounts of lipids (Mandal and Mallick, 2009; Ho et al., 2012; Breuer et al., 2013b; Remmers et al., 2017). T. obliquus reached a maximum triacylglyceride (TAG) yield on light of $0.14 \mathrm{~g}_{\mathrm{TAG}} \cdot \mathrm{mol}_{\mathrm{ph}}$ ${ }^{1}$ and a final TAG content of $0.45 \mathrm{~g} \cdot \mathrm{gDW}^{-1}$ under batch nitrogen starvation conditions (Breuer et al., 2014).

At this moment the bulk production of microalgal products is not economically feasible, which is partly due to cultivation costs (Ruiz et al., 2016). The production costs can be lowered by increasing the yield on light for a desired product. Strategies to accomplish this involve the steering of algal metabolism towards the product of choice and increasing the efficiency with which the light is used. However, finding the right pathways and enzymes to target in order to reach this is difficult due to a lack of understanding of algal metabolism. Genome-scale metabolic models are a powerful tool for obtaining a better understanding of metabolism and are used for several applications, such as finding targets for metabolic engineering or elucidating regulatory mechanisms in a metabolic network (Feist and Palsson, 2010).

Several metabolic models can be found in literature for eukaryotic microalgae. A first reconstruction of the metabolic network of Chlorella sorokiniana contained 67 reactions and 61 metabolites, with a low level of compartmentalisation as little was known regarding localization (Yang et al., 2000). With the sequence and annotation of the genome of the model microalgae Chlamydomonas reinhardtii (Merchant et al., 2007) a large amount of information about the metabolism became available and metabolic models were developed (Boyle and Morgan, 2009; Manichaikul et al., 2009; Chang et al., 2011; Cogne et al., 2011; Dal'Molin et al., 2011; Kliphuis et al., 2012; Imam et al., 2015). These models had a higher level of compartmentalisation, as well as a higher number of reactions and metabolites. 
The problem with the genome-scale models is that both the annotation and prediction in which compartment a protein ends up, is poor (Reijnders et al., 2014). As an alternative to the genome scale models, core models are developed containing the primary metabolism (Kayser et al., 2005; Boyle and Morgan, 2009; Kliphuis et al., 2012; Muthuraj et al., 2013). These models differ in their representation of internal metabolism, as all the reactions that are annotated in the genome are present in the genome-scale models. However, both models can correctly make predictions, as shown for E. coli with a genome scale metabolic model (Feist et al., 2007) as well as a core model (Kayser et al., 2005). Additionally, the primary metabolism is usually well annotated and information on localisation of metabolic pathways can be obtained from literature.

As for genome-scale models, the amount of energy needed for making biomass (growth-associated maintenance, $\mathrm{K}_{\mathrm{x}}$ ) and for maintenance (non-growth-associated maintenance, $\mathrm{m}_{\text {ATP }}$ ) are two important unknown parameters that must be determined experimentally. A common strategy to estimate the energy parameters is using a chemostat set-up. However, if for both systems the maximum amount of light per cell is the same, the batch approach in principle covers the same range of growth rates as the chemostat approach. For algae growing under light-limited batch cultures, the available light per cell slowly decreases due to the growth of the algae themselves. Thus, the batch approach allows to go slowly through a whole range of different specific growth rates throughout the cultivation, whereas in a chemostat usually a limited number of growth rates is tested.

In this paper we developed a core model for $T$. obliquus. We verified whether the genes responsible for the reactions in the model are present in the genome as published by Carreres et al. (2017). Then we determined experimentally the energy parameters for maintenance and biomass formation from batch cultivations and included them in the model. Finally, the model was used to predict the theoretical yields of biomass, starch and triacylglycerides (TAG) on light. 


\subsection{Materials and methods}

\subsubsection{Model development and Flux Balance Analysis (FBA)}

An organism's metabolism can be described by a set of stoichiometric reactions (Stephanopoulos et al., 1998). We constructed a de novo metabolic network for Tetradesmus obliquus where the reactions were included in either the chloroplast, cytosol or mitochondria based on information from text books and literature (Hoefnagel et al., 1998; Laloi, 1999; Atteia et al., 2009; Stern, 2009; Chang et al., 2011). Afterwards, the transport steps between the compartments were added. Finally, we checked whether the genes responsible for the included reactions were present in the genome reported by Carreres et al. (2017).

After the biochemical network was developed, the intracellular fluxes of the network were studied using Flux Balance Analysis (FBA). An objective function was set and the solution space was narrowed down by including constraints. Such constraints included the quasi steady-state constrain where the accumulation of intermediate metabolites as well as the depletion is assumed to be zero (this is, no dynamics for the intracellular metabolites), setting boundaries for measured reactions, and restricting the directionality of reactions based on thermodynamics. This is mathematically represented as:

$$
\begin{array}{ll}
\text { Objective function: } & \max (c \cdot v) \\
\text { Constraints: } & S \cdot v=0 \\
& L B \leq v \leq U B
\end{array}
$$

Where $S$ is the stoichiometric matrix $S$ (where $S_{i j}$ contains the stoichiometric coefficient of compound $i$ in reaction $j), c$ is a row vector which defines the objective function, and $L B$ and $U B$ are the lower and upper boundaries for the reaction rates of the reaction vector $v$. To estimate the energy parameters, the ATP production was used as an objective function and the specific growth rates calculated on different time points (section 6.2.5) and the light supply rate (section 6.2.6) were measured and used to constrain the solution space. The network contained several circulations and therefore a unique solution for the flux distribution cannot be calculated. Therefore, we used the 
geometric mean to single out a unique solution. The geometric mean represents a unique solution in the solution space, which minimizes the total flux in the cell ,while satisfying the objective function, removing thermodynamically infeasible cycles and imposed constraints (Smallbone and Simeonidis, 2009).

In silico simulations were calculated using COBRA Toolbox (Becker et al., 2007; Schellenberger et al., 2011) with the 'glpk' solver in MATLAB R2015b version 8.6 (The MathWorks Inc, USA).

\subsubsection{Energy parameters}

The growth associated maintenance $\left(\mathrm{K}_{\mathrm{x}}\right)$ and the non-growth associated maintenance ( $\left.m_{\text {ATP }}\right)$ parameters were calculated from experimental data applied to the model. For this calculation, the ATP balance is written as:

$$
\sum \mathrm{s}_{\mathrm{i}}^{\mathrm{ATP}} \cdot \mathrm{v}_{\mathrm{i}}-\mathrm{K}_{\mathrm{x}} \cdot \mu-\mathrm{m}_{\mathrm{ATP}}=0
$$

The first term in the equation represents the net production of ATP in the network excluding ATP used for growth and maintenance. Here $\mathrm{si}^{\mathrm{ATP}}$ is the stoichiometric coefficient of ATP in reaction $i$ and $v_{i}$ is the flux through reaction $i$. ATP production from oxidative phosphorylation and the light reactions are included in this term. The stoichiometry for the oxidation of $\mathrm{NADH}$ and $\mathrm{FADH}_{2}$ in the oxidative phosphorylation is defined based on the $\mathrm{P} / \mathrm{O}$ ratio (ratio of ATP synthesized per $1 / 2$ mole of $\mathrm{O}_{2}$ reduced to water). A P/O ratio of 2.5 and 1.5 is generally accepted for $\mathrm{NADH}$ and $\mathrm{FADH}_{2}$, respectively, and was used for the model. With respect to ATP generation from light, the two most important processes that result in ATP and/or NADPH production were considered. During the linear electron transport (LET), according to the Z-scheme of photosynthesis, 3 ATP and 2 NADPH are produced for every 8 photons absorbed. Additionally, the cyclic electron transport (CET) yields 1 ATP per 2 photons absorbed. Optimal photosynthetic efficiency of 8 photons for 3 ATP and 2 NADPH is in practice never achieved, possibly because always a certain minimal amount of CET occurs. Therefore a minimum requirement of 10 photons is assumed for LET to produce 3 ATP and 2 NADPH (Kliphuis et al., 2012; Klok, 2013). $K_{x}$ represents the ATP requirement for the formation of biomass from biopolymers (in $\mathrm{mmol}_{\mathrm{ATP}} \cdot \mathrm{gDW}^{-1}$ ). This constant includes the processes that use ATP and are not defined as reactions in the network (e.g. the 
assembly of biopolymers like protein and lipids into functional cells). $\mu$ stands for the specific growth rate calculated on different time points $\left(h^{-1}\right)$. The final term $m_{\text {ATP }}$ (in $\mathrm{mmol}_{\mathrm{ATP}} \cdot \mathrm{g}_{\mathrm{DW}}{ }^{-1} \cdot \mathrm{h}^{-1}$ ) refers to the maintenance requirements of the cell, which vary depending on the species and culture conditions.

With the $\mathrm{P} / \mathrm{O}$ ratio and the stoichiometry of the light reactions known, the first term can be calculated if the flux distribution is known. This term represents the net specific ATP production rate ( $q_{\text {ATP }}$ ) in the network. Then Eq. 6.1 can be written as:

$$
\mathrm{q}_{\mathrm{ATP}}=\mathrm{K}_{\mathrm{x}} \cdot \mu+\mathrm{m}_{\mathrm{ATP}}
$$

The parameters $K_{x}$ and $m_{\text {ATP }}$ can now be experimentally estimated by plotting the simulated $q_{\text {ATP }}$ against the specific growth rates calculated on different time points $(\mu)$.

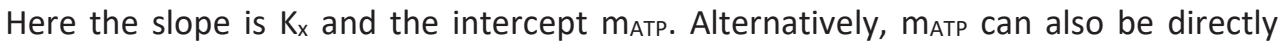
calculated from the qATP during the stationary phase $(\mu=0)$ of the batch culture.

To estimate $K_{x}$ and $m_{A T P}$, a series of batch experiments were carried out under nutrient replete conditions and at low light intensity. Samples were taken at different time points of the batch cultivations to determine a wide range of specific growth rates. The maximum ATP production rate ( $\mathrm{q}_{\text {ATP }}$ ) was calculated using FBA. For each growth rate a separate biomass equation was used that matched the measured biomass composition at that specific growth rate.

\subsubsection{Strains, pre-culture conditions and cultivation medium}

Wild-type Tetradesmus obliquus UTEX 393 was obtained from the Culture Collection of Algae, University of Texas. Pre-cultures were grown in filter sterilized (pore size $0.2 \mu \mathrm{m}$ ) freshwater medium $\left(\mathrm{KNO}_{3} 9.9 \mathrm{mM}, \mathrm{Na}_{2} \mathrm{SO}_{4} 0.7 \mathrm{mM}\right.$, HEPES $100.0 \mathrm{mM}$. MgSO${ }_{4} \cdot 7 \mathrm{H}_{2} \mathrm{O}$ $0.6 \mathrm{mM}, \mathrm{CaCl}_{2} \cdot 2 \mathrm{H}_{2} \mathrm{O} 0.5 \mathrm{mM}, \mathrm{K}_{2} \mathrm{HPO}_{4} 2.5 \mathrm{mM}, \mathrm{NaHCO}_{3} 10.0 \mathrm{mM}, \mathrm{Na}_{2} \mathrm{EDTA}_{2} \cdot 2 \mathrm{H}_{2} \mathrm{O}$ $80.0 \mu \mathrm{M}, \mathrm{MnCl}_{2} \cdot 4 \mathrm{H}_{2} \mathrm{O} 19.0 \mu \mathrm{M}, \mathrm{ZnSO}_{4} \cdot 7 \mathrm{H}_{2} \mathrm{O} 4.0 \mu \mathrm{M}, \mathrm{CoCl}_{2} \cdot 6 \mathrm{H}_{2} \mathrm{O} 1.2 \mu \mathrm{M}, \mathrm{CuSO}_{4} \cdot 5 \mathrm{H}_{2} \mathrm{O}$ $1.3 \mu \mathrm{M}, \mathrm{Na}_{2} \mathrm{MoO}_{4} \cdot 2 \mathrm{H}_{2} \mathrm{O} 0.1 \mu \mathrm{M}$, NaFeEDTA $27.8 \mu \mathrm{M}$, Biotin $0.1 \mu \mathrm{M}$, vitamin B1 $3.3 \mu \mathrm{M}$, vitamin B12 $0.1 \mu \mathrm{M}$ ) (Breuer et al., 2013b), as described by León-Saiki et al. (2017). 


\subsubsection{Batch reactor experiments}

Batch cultures of $T$. obliquus were conducted in a sterile flat panel airlift-loop reactor with a working volume of $1.8 \mathrm{~L}$ and a light path of $0.02 \mathrm{~m}$ (Labfors $5 \mathrm{Lux}$, Infors HT, Switzerland). Reactors were inoculated to an optical density (OD $\left.{ }_{750}\right)$ of 0.01 and were continuously illuminated by a panel with 260 LED lamps. Reactor medium composition was the same as for pre-cultures, with the differences that HEPES and $\mathrm{NaHCO}_{3}$ were not included. $\mathrm{KNO}_{3}$ initial concentration was $1 \mathrm{~g} \cdot \mathrm{L}^{-1}$ for replicate $\# 1,7.5 \mathrm{~g} \cdot \mathrm{L}^{-1}$ for replicates \#2-4, and $10 \mathrm{~g} \cdot \mathrm{L}^{-1}$ for replicates \#5-8 to prevent nitrogen limitation. For replicate $\# 1$, additional $\mathrm{KNO}_{3}$ was supplied to the culture when nitrogen was fully consumed. The incident light intensity was $90 \mu \mathrm{mol} \cdot \mathrm{m}^{-2} \cdot \mathrm{s}^{-1}$ (Breuer et al., 2015a), to ensure that the photosystems were working at maximum efficiency by avoiding light saturation and preventing damage and dissipation of energy (Baker et al., 2007; Kliphuis et al., 2012; Breuer et al., 2015a). A 2\% v/v antifoam solution (Antifoam B, Baker, The Netherlands) was supplied when foam build-up was observed.

\subsubsection{Specific growth rate}

The microalgal cultures experienced different growth rates throughout a batch cultivation. During the exponential growth phase, the increase in biomass per unit of time is proportional to the biomass present in the culture at the beginning of any unit of time (Michelle Wood et al., 2005). Maximum growth rate during the exponential phase, $\mu_{\max }\left(\right.$ in $\left.\mathrm{h}^{-1}\right)$, was calculated by performing a logarithmic linearization as follows:

$$
\ln \left(C_{x}\right)=\ln \left(C_{x_{0}}\right)+\mu_{\max } \cdot t
$$

where $C_{x}$ is the biomass concentration (in $\mathrm{g} \cdot \mathrm{L}^{-1}$ ) at time $t$ (in $\mathrm{h}$ ), and $\mathrm{C}_{\mathrm{x}_{0}}$ is the biomass concentration at the beginning (in $\left.\mathrm{g} \cdot \mathrm{L}^{-1}\right)$.

During the linear phase, the specific growth rate $\mu$ (in $\mathrm{h}^{-1}$ ) was calculated on different time points by dividing the slope between three measured biomass concentration points by the biomass concentration $\mathrm{C}_{\mathrm{x}}\left(\right.$ in $\left.\mathrm{g} \cdot \mathrm{L}^{-1}\right)$ :

$$
\frac{d C_{x}}{d t}=\mu \cdot C_{x} \rightarrow \mu=\frac{\frac{d C_{x}}{d t}}{C_{x}}=\frac{\frac{C_{x}(t)-C_{\chi}(t-1)}{\Delta t_{1}}+\frac{C_{x}(t+1)-C_{\chi}(t)}{\Delta t_{2}}}{2 C_{x}}
$$




\subsubsection{Specific light supply rate}

The light intake rate was calculated for each specific growth rate $\mu$ using the following equation:

$$
r_{E x}=\frac{P F D_{a b s} \cdot A_{p b r}}{C_{x} \cdot V_{p b r}}=\frac{P F D_{a b s}}{C_{x} \cdot Z_{p b r}}
$$

Where $\mathrm{r}_{\mathrm{Ex}}$ is the light supply rate per amount of biomass (in $\mathrm{mmol}_{\mathrm{ph}} \cdot \mathrm{g}_{\mathrm{DW}}{ }^{-1} \cdot \mathrm{h}^{-1}$ ), $\mathrm{PFD}_{\mathrm{abs}}$ is the absorbed photon flux density (in $\mathrm{mmol}_{\mathrm{ph}} \cdot \mathrm{m}^{-2} \cdot \mathrm{h}^{-1}$ ), $\mathrm{Apbr}_{\mathrm{pr}}$ is the illuminated area of the photobioreactor (in $\mathrm{m}^{2}$ ), $\mathrm{C}_{\mathrm{x}}$ is the biomass concentration (in $\mathrm{g} \cdot \mathrm{m}^{-3}$ ), $\mathrm{V}_{\mathrm{pbr}}$ is the working volume of the photobioreactor $\left(\mathrm{m}^{3}\right)$, and $Z_{p b r}$ is the light path (in $\mathrm{m}$ ). The PFDabs was estimated by subtracting the corrected light leaving the reactor $\left(P F_{\text {out }}\right.$, in $\mathrm{mmol}_{\mathrm{ph}} \cdot \mathrm{m}^{-}$ $\left.{ }^{2} \cdot \mathrm{h}^{-1}\right)$ to the incident light $\left(\mathrm{PDF}_{\mathrm{in}}\right.$, in $\left.\mathrm{mmol}_{\mathrm{ph}} \cdot \mathrm{m}^{-2} \cdot \mathrm{h}^{-1}\right)$ as described by Mulders et al. (2014). Light on the rear of the reactor was manually measured with a light meter (LI250, LICOR, USA) and corrected for the dissipation by the water jacket. In addition, the relationship between the specific light supply rate $\left(r_{E x}\right)$ and the specific growth rate at different time points $(\mu)$ can be used to estimate the biomass yield on light $Y_{x / p h}$ (in $\mathrm{gDw}_{\mathrm{DW}} \cdot \mathrm{mmol}_{\mathrm{ph}}{ }^{-1}$ ), as used by Zijffers et al. (2010) based on the model of Pirt (1965):

$$
r_{E x}=\frac{\mu}{Y_{x, p h}}+m_{E}
$$

where $m_{E}$ is the photon maintenance requirement (in $\mathrm{mmol}_{\mathrm{ph}} \cdot \mathrm{g}_{\mathrm{DW}}{ }^{-1} \cdot \mathrm{h}^{-1}$ ). The maintenance energy is the energy required for functions other than production of biomass (Pirt, 1965).

\subsubsection{Analyses}

Dry weight (DW) concentration was determined according to Kliphuis et al. (2012). Starch content was measured as described by de Jaeger et al. (2014) using $5 \mathrm{mg}$ of freeze-dried biomass and a total starch kit (Megazyme, Ireland). Triacylglycerol (TAG) content and polar lipid content were quantified as described by Remmers et al. (2017) using $7 \mathrm{mg}$ of freeze-dried biomass and 1,2-dipentadecanoyl-sn-glycero-3-phospho(1'-rac-glycerol) (sodium salt) (840446, Avanti Polar Lipids Inc) and tripentadecanoin (T4257, Sigma Aldrich) as internal standards. Total carbohydrates were measured by pre-treating $1 \mathrm{mg}$ of freeze dried biomass with $0.5 \mathrm{~mL}$ of $2.5 \mathrm{M} \mathrm{HCl}$, followed by 
incubation in boiling water for $3 \mathrm{~h}$. After this, samples were cooled down to room temperature and neutralized with $0.5 \mathrm{~mL}$ of $2.5 \mathrm{M} \mathrm{NaOH}$. Quantification was done using a $5 \%(\mathrm{v} / \mathrm{v})$ solution of phenol:water and concentrated $\mathrm{H}_{2} \mathrm{SO}_{4}$ according to DuBois et al. (1956) and Hebert et al. (1971). Absorbance was measured at $483 \mathrm{~nm}$. A $1 \mathrm{~g} \cdot \mathrm{L}^{-1}$ solution of glucose was used as standard and starch was used as control. Ash content was measured as described by Kliphuis et al. (2012) using at least 38 mg of freeze-dried biomass. Pigment (chlorophyll and carotene in $\mu \mathrm{g} \cdot \mathrm{mL}^{-1}$ ) content were extracted using methanol at $60^{\circ} \mathrm{C}$ for $30 \mathrm{~min}$. Absorbance was measured at 470, 652 and $665 \mathrm{~nm}$. The assay was done in triplicate. Arnon's equations were used to calculate the pigment concentration (Lichtenthaler, 1987):

$$
\begin{aligned}
& C h l_{a}=16.72 \cdot O D_{665}-9.16 \cdot O D_{652} \\
& C h l_{b}=34.09 \cdot O D_{652}-15.28 \cdot O D_{665} \\
& C h l_{\text {total }}=C h l_{a}+C h l_{b} \\
& \operatorname{Car}_{\text {total }}=\frac{1000 \cdot O D_{470}-1.63 \cdot C h l_{a}-104.96 \cdot C h l_{b}}{221}
\end{aligned}
$$

Where $\mathrm{Chl}_{\mathrm{a}}$ stands for chlorophyll a, Chl $\mathrm{b}_{\mathrm{b}}$ for chlorophyll b, $\mathrm{Ch}_{\text {total }}$ for chlorophyll total and Car total for carotene total.

\subsubsection{Biomass equation coefficients under different growth rates}

To calculate the biomass equation coefficients, first the biomass composition for all the measured components (carbohydrates $(\mathrm{CHO})$, polar lipids $(\mathrm{PL}), \mathrm{TAG}$, proteins, chlorophyll (chl), carotenes (car) and ashes) was normalized to $93.8 \%$ (100\% minus DNA and RNA content). DNA and RNA content were not experimentally measured but were taken from the literature $\left(0.002 \mathrm{~g} \cdot \mathrm{gDW}^{-1}\right.$ for DNA and $0.06 \mathrm{~g} \cdot \mathrm{g}_{\mathrm{DW}}{ }^{-1}$ for RNA) (Kliphuis et al., 2012). After normalization, the biomass equation coefficients (in $\mathrm{mmol} \cdot \mathrm{gDW}^{-1}$ ) were calculated by dividing the fraction of the component by the molecular weight of each component (CHO: $162.1 \mathrm{~g}_{\mathrm{CO}} \cdot \mathrm{mol}^{-1}, \mathrm{PL}: 757.5 \mathrm{gPL} \cdot \mathrm{mol}^{-1}$, TAG: $864.3 \mathrm{~g}_{\mathrm{TAG}} \cdot \mathrm{mol}^{-1}$, proteins: $106.4 \mathrm{~g}_{\mathrm{Prot}} \cdot \mathrm{mol}^{-1}$, chl: $869.2 \mathrm{gchl} \cdot \mathrm{mol}^{-1}, \mathrm{car} 536.9 \mathrm{~g} \mathrm{Car} \cdot \mathrm{mol}^{-1}$, RNA: $339.2 \mathrm{~g}_{\mathrm{RNA}} \cdot \mathrm{mol}^{-1}$, and DNA: $\left.356.0 \mathrm{~g}_{\mathrm{DNA}} \cdot \mathrm{mol}^{-1}\right)$. Molecular weights of the different macromolecules were calculated based on the elemental composition of the 
compound in the model. The biomass equation defined in this way produces $1 \mathrm{~g}$ of biomass dry weight.

The average biomass equation (in $\mathrm{mmol} \cdot \mathrm{g}_{\mathrm{DW}}{ }^{-1}$ ) used to calculate the theoretical yields was formulated using the biomass coefficients of the specific growth rates calculated for time points higher than $0.01 \mathrm{~h}^{-1}$, where the biomass composition remained almost constant. As the biomass was set to produce $1 \mathrm{~g}_{\mathrm{DW}}$, the flux resulting from the biomass synthesis equation is the specific growth rate and has units of $h^{-1}$.

\subsubsection{Theoretical yields of biomass, starch, and TAG on light}

The theoretical biomass yield on light was estimated by optimizing the biomass production divided by the photons supplied. The TAG and starch yield on light were estimated by optimizing the TAG or starch content, and dividing this by their molecular weight and the photons supplied. The molecular weights used were $147.7 \mathrm{~g} \cdot \mathrm{mol}^{-1}$ for biomass, $864.3 \mathrm{~g} \cdot \mathrm{mol}^{-1}$ for TAG and $162.1 \mathrm{~g} \cdot \mathrm{mol}^{-1} \mathrm{for}$ starch. The yield of TAG on starch was calculated by setting the lower boundary of the starch exchange rate and the light supply rate to -1 and 0 , respectively. TAG was used as the function to be optimized. The maintenance energy ( $m_{\text {ATP }}$ ) was set to zero for these estimations, as otherwise the yield becomes dependent on the light intensity used.

\subsubsection{Dissolved nitrate and phosphate}

The dissolved nitrate concentration was measured using a Seal Analytical AQ2 nutrient analyser (SEAL Analytical Inc., USA) as explained by Benvenuti et al. (2015). Phosphate concentration was also measured using a Seal Analytical AQ2 nutrient analyser (SEAL Analytical Inc., USA). The method is based on the reaction with acidic molybdate in the presence of antimony forms an antimony phospho-molybdate complex which is reduced by ascorbic acid to an intensely blue complex: phosphomolybdenum blue. The absorbance of this complex is measured spectrophotometrically at $880 \mathrm{~nm}$. Dilutions and standards were prepared according to manufacturer's instructions. 


\subsubsection{Statistical analysis}

Eight batch runs were performed at low light intensity to estimate the energy parameters. The maintenance requirement ( $m_{\text {ATP }}$ ) was calculated based on the model as the intercept from plotting the specific growth rates calculated on different time points and the $q_{\text {ATP }}(\mathrm{N}=65$ measurements). From these 65 measurements points, 15 corresponded to the stationary phase ( $\mathrm{N}=15$ measurements) and were used directly to estimate the maintenance energy. A Student's T-test with a significant level of $p<0.05$ was used to evaluate the difference between both values.

\subsection{Results and Discussion}

\subsubsection{Metabolic model construction}

In this work, a network of metabolic reactions describing the primary metabolism of Tetradesmus obliquus and their subcellular location was defined based on literature (Hoefnagel et al., 1998; Laloi, 1999; Atteia et al., 2009; Stern, 2009; Chang et al., 2011) and biochemical text books (Alberts et al., 2002).

In total, the metabolic network consists of 351 reactions with 183 metabolites distributed over 4 compartments: cytosol, chloroplast, mitochondria and extracellular space (Supplementary Files S6.1 and S6.2). A total of 35 reactions were placed in the mitochondria, including reactions of the photorespiratory, nitrate, glyoxylate, amino acid, folate, Tricarboxylic acid (TCA) cycle, and oxidative phosphorylation pathways. For the chloroplast, 98 reactions were included. The reactions were part of the following pathways: photosynthesis, Calvin cycle, photorespiration, starch metabolism, nitrogen assimilation, sulphur assimilation, amino acid metabolism, and fatty acid biosynthesis. In the cytosol, reactions from the pentose phosphate pathway, fatty acid biosynthesis, purine and pyrimidine metabolism were included, together with reactions from other compartments (e.g. glyoxisome). Finally, transport reactions between the compartments were added based on literature or requirement to make a functional model. The reactions, as well as the EC numbers for the enzymes, can be found in Supplementary File S6.1. Reactions were grouped into different metabolic pathways to facilitate the analysis of the flux distributions predicted by the model. 
All enzymes were present in the genome, except for 16 reactions (Table 6.1). Their absence could be associated with a poor annotation of the genome, as annotation from microalgae is mainly based on higher plants (Reijnders et al., 2014; Reijnders et al., 2015). Of these 16 missing reactions, the enzyme from reactions 162 (desaturase) is part of the fatty acid synthesis. This enzyme is responsible for introducing double bounds in fatty acids. Even though no evidence of its presence could be found in the genome, the enzyme was included in the model as $T$. obliquus is known to be able to perform these reactions. The other reactions are involved in the Calvin cycle (28), glycolysis (30), photorespiration (39 and 47), TCA cycle (78), amino acid synthesis (106, 128, 131), fermentation (150), fatty acid biosynthesis (154-156), and purine and pyrimidine metabolism (176, 180 and 187). These reactions were included as they are needed for the model to support growth.

\subsubsection{Batch growth and biomass composition}

Eight batch runs were performed at low light intensity to estimate the energy parameters for maintenance ( $\mathrm{m}_{\mathrm{ATP}}$ ) and formation of biomass $\left(\mathrm{K}_{\mathrm{x}}\right)$. Three of them were focused on the exponential and linear phase and the other five were carried out to the stationary phase. Commonly, a batch growth curve for algae can be divided into five phases: lag, exponential growth, linear growth, stationary, and death phase. Growth profiles are shown in Figure 6.1A. We observed that the growth was more or less exponential until the biomass concentration $\left(C_{x}\right)$ reached $0.14 \mathrm{gDw}_{\mathrm{D}} \mathrm{L}^{-1}$ (approximately $72 \mathrm{~h}$ ). After this point, the outgoing light decreased inverse proportionally to the increase in biomass concentration, reaching full light absorption approximately after $120 \mathrm{~h}\left(\mathrm{C}_{\mathrm{x}}\right.$ of approximately $\left.0.71 \mathrm{gDw} \cdot \mathrm{L}^{-1}\right)$. From there on, the increase in biomass was approximately linear in time to about $6 \mathrm{gDw}_{\mathrm{DW}} \mathrm{L}^{-1}$. After this point the increase in biomass gradually decreased to zero and the biomass concentration became constant (stationary phase). The range of specific growth rates calculated on different time points obtained from the batch runs was from 0 (stationary phase) to $0.053 \mathrm{~h}^{-1}$ (exponential phase). The differences in final biomass concentration (from 7 to 10 $\mathrm{gDW}_{\mathrm{DW}} \cdot \mathrm{L}^{-1}$ ) between the batch runs may be related to differences in mixing patterns and biomass sedimentation or small differences in maintenance requirements between the cultures (see below). 
Table 6.1. List of missing enzymes in the genome of Tetradesmus obliquus

\begin{tabular}{|c|c|c|c|}
\hline \# & Reaction & Enzyme name & $\begin{array}{c}\text { E.C. } \\
\text { number }\end{array}$ \\
\hline 28 & $\begin{array}{l}\text { MAL[h] + NADP[h] } \Leftrightarrow=>\mathrm{CO} 2[\mathrm{~h}]+\mathrm{NADPH}[\mathrm{h}]+ \\
\operatorname{PYR}[\mathrm{h}]\end{array}$ & Malate dehydrogenase & 1.1 .1 .40 \\
\hline 30 & $\mathrm{~F} 6 \mathrm{P}[\mathrm{h}] \Leftrightarrow=>\mathrm{G} 6 \mathrm{P}[\mathrm{h}]$ & $\begin{array}{l}\text { Glucose-6-phosphate } \\
\text { isomerase }\end{array}$ & 5.3 .1 .9 \\
\hline 39 & $\begin{array}{l}\mathrm{NAD}[\mathrm{m}]+\text { glycolate }[\mathrm{m}]->\mathrm{GLYX}[\mathrm{m}]+\mathrm{H}[\mathrm{m}]+ \\
\mathrm{NADH}[\mathrm{m}]\end{array}$ & $\begin{array}{l}\text { Glyoxylate reductase or } \\
\text { glycolate dehydrogenase }\end{array}$ & $\begin{array}{l}1.1 .1 .26 \\
1.1 .99 .14\end{array}$ \\
\hline 47 & $\mathrm{GA}[\mathrm{c}]+\mathrm{H}[\mathrm{c}]+\mathrm{NADPH}[\mathrm{c}] \Leftrightarrow=\mathrm{GLYC}[\mathrm{c}]+\mathrm{NADP}[\mathrm{c}]$ & Alcohol dehydrogenase & 1.1.1.2 \\
\hline 78 & $\operatorname{ATP}[c]+\mathrm{OXA}[c] \rightarrow \operatorname{ADP}[c]+\mathrm{CO} 2[\mathrm{c}]+\mathrm{PEP}[\mathrm{c}]$ & $\begin{array}{l}\text { Phosphoenolpyruvate } \\
\text { carboxykinase }\end{array}$ & 4.1.1.49 \\
\hline 106 & $\begin{array}{l}\text { ATP }[h]+E 4 P[h]+N A D P H[h]+2 P E P[h]-> \\
A D P[h]+C H O[h]+N A D P[h]+4 P i[h]\end{array}$ & Chorismate synthase & 4.2.3.5 \\
\hline 128 & $\begin{array}{l}\mathrm{ASA}[\mathrm{c}]+\mathrm{GLU}[\mathrm{c}]+2 \mathrm{H}[\mathrm{c}]+\mathrm{NADPH}[\mathrm{c}]+\mathrm{PYR}[\mathrm{c}] \\
<=>\mathrm{AKG}[\mathrm{c}]+\mathrm{DAP}[\mathrm{c}]+\mathrm{H} 2 \mathrm{O}[\mathrm{c}]+\mathrm{NADP}[\mathrm{c}]\end{array}$ & $\begin{array}{l}\text { Diaminopimelate } \\
\text { epimerase }\end{array}$ & 5.1.1.7 \\
\hline 131 & $\begin{array}{l}\text { SUCCOA[h] + HSER[h] } \Leftrightarrow=>\operatorname{CoA}[h]+ \\
\text { OSUCHSER[h] }\end{array}$ & $\begin{array}{c}\text { Homoserine O- } \\
\text { succinyltransferase }\end{array}$ & 2.3.1.46 \\
\hline 150 & $\begin{array}{l}\mathrm{MAL}[\mathrm{m}]+\mathrm{NAD}[\mathrm{m}] \Leftrightarrow=>\mathrm{CO} 2[\mathrm{~m}]+\mathrm{NADH}[\mathrm{m}]+ \\
\operatorname{PYR}[\mathrm{m}]\end{array}$ & Malate dehydrogenase & 1.1.1.39 \\
\hline 154 & $\begin{array}{l}\text { AcACP }[\mathrm{h}]+12 \mathrm{H}[\mathrm{h}]+6 \mathrm{MalACP}[\mathrm{h}]+12 \\
\mathrm{NADPH}[\mathrm{h}]->6 \mathrm{ACP}[\mathrm{h}]+\mathrm{C} 140 \mathrm{ACP}[\mathrm{h}]+6 \mathrm{CO} 2[\mathrm{~h}] \\
+6 \mathrm{H} 2 \mathrm{O}[\mathrm{h}]+12 \mathrm{NADP}[\mathrm{h}]\end{array}$ & $\begin{array}{l}\text { Enoyl-[acyl-carrier- } \\
\text { protein] reductase }\end{array}$ & 1.3.1.10 \\
\hline 155 & $\begin{array}{l}\text { AcACP }[\mathrm{h}]+14 \mathrm{H}[\mathrm{h}]+7 \mathrm{MalACP}[\mathrm{h}]+14 \\
\mathrm{NADPH}[\mathrm{h}]->7 \mathrm{ACP}[\mathrm{h}]+\mathrm{C} 160 \mathrm{ACP}[\mathrm{h}]+7 \mathrm{CO} 2[\mathrm{~h}] \\
+7 \mathrm{H} 2 \mathrm{O}[\mathrm{h}]+14 \mathrm{NADP}[\mathrm{h}]\end{array}$ & $\begin{array}{l}\text { Enoyl-[acyl-carrier- } \\
\text { protein] reductase }\end{array}$ & 1.3.1.10 \\
\hline 156 & $\begin{array}{l}\text { AcACP }[\mathrm{h}]+16 \mathrm{H}[\mathrm{h}]+8 \mathrm{MalACP}[\mathrm{h}]+16 \\
\mathrm{NADPH}[\mathrm{h}]->8 \mathrm{ACP}[\mathrm{h}]+\mathrm{C} 180 \mathrm{ACP}[\mathrm{h}]+8 \mathrm{CO} 2[\mathrm{~h}] \\
+8 \mathrm{H} 2 \mathrm{O}[\mathrm{h}]+16 \mathrm{NADP}[\mathrm{h}]\end{array}$ & $\begin{array}{l}\text { Enoyl-[acyl-carrier- } \\
\text { protein] reductase }\end{array}$ & 1.3.1.10 \\
\hline 162 & $\begin{array}{l}\mathrm{C} 181 \mathrm{CoA}[\mathrm{c}]+\mathrm{H}[\mathrm{c}]+\mathrm{NADH}[\mathrm{c}]+\mathrm{O} 2[\mathrm{c}] \Leftrightarrow=> \\
\mathrm{C} 182 \mathrm{CoA}[\mathrm{c}]+2 \mathrm{H} 2 \mathrm{O}[\mathrm{c}]+\mathrm{NAD}[\mathrm{c}]\end{array}$ & Desaturase & \\
\hline 176 & $\begin{array}{l}\mathrm{ASP}[\mathrm{c}]+\mathrm{GTP}[\mathrm{c}]+\mathrm{IMP}[\mathrm{c}] \Leftrightarrow=>\mathrm{AMP}[\mathrm{c}]+\mathrm{FUM}[\mathrm{c}] \\
+\mathrm{GDP}[\mathrm{c}]+\mathrm{Pi}[\mathrm{c}]\end{array}$ & $\begin{array}{l}\text { Adenylosuccinate } \\
\text { synthase }\end{array}$ & 6.3.4.4 \\
\hline 180 & $\begin{array}{l}\mathrm{ASP}[\mathrm{c}]+\mathrm{CaP}[\mathrm{c}]+\mathrm{H}[\mathrm{c}]+\mathrm{O} 2[\mathrm{c}]+\mathrm{PRPP}[\mathrm{c}] \Leftrightarrow=> \\
\mathrm{CO} 2[\mathrm{c}]+\mathrm{H} 2 \mathrm{O} 2[\mathrm{c}]+\mathrm{H} 2 \mathrm{O}[\mathrm{c}]+\mathrm{PPi}[\mathrm{c}]+\mathrm{Pi}[\mathrm{c}]+ \\
\mathrm{UMP}[\mathrm{c}]\end{array}$ & $\begin{array}{l}\text { Dihydroorotate } \\
\text { dehydrogenase }\end{array}$ & 1.3.1.14 \\
\hline 187 & $\begin{array}{l}\operatorname{METHF}[c]+\operatorname{UDP}[c]+\operatorname{trdrd}[c]->\operatorname{DHF}[c]+ \\
\mathrm{H} 2 \mathrm{O}[\mathrm{c}]+\mathrm{dTDP}[\mathrm{c}]+\operatorname{trdox}[\mathrm{c}]\end{array}$ & $\begin{array}{l}\text { Nucleoside-phosphate } \\
\text { kinase }\end{array}$ & 2.7.4.4 \\
\hline
\end{tabular}



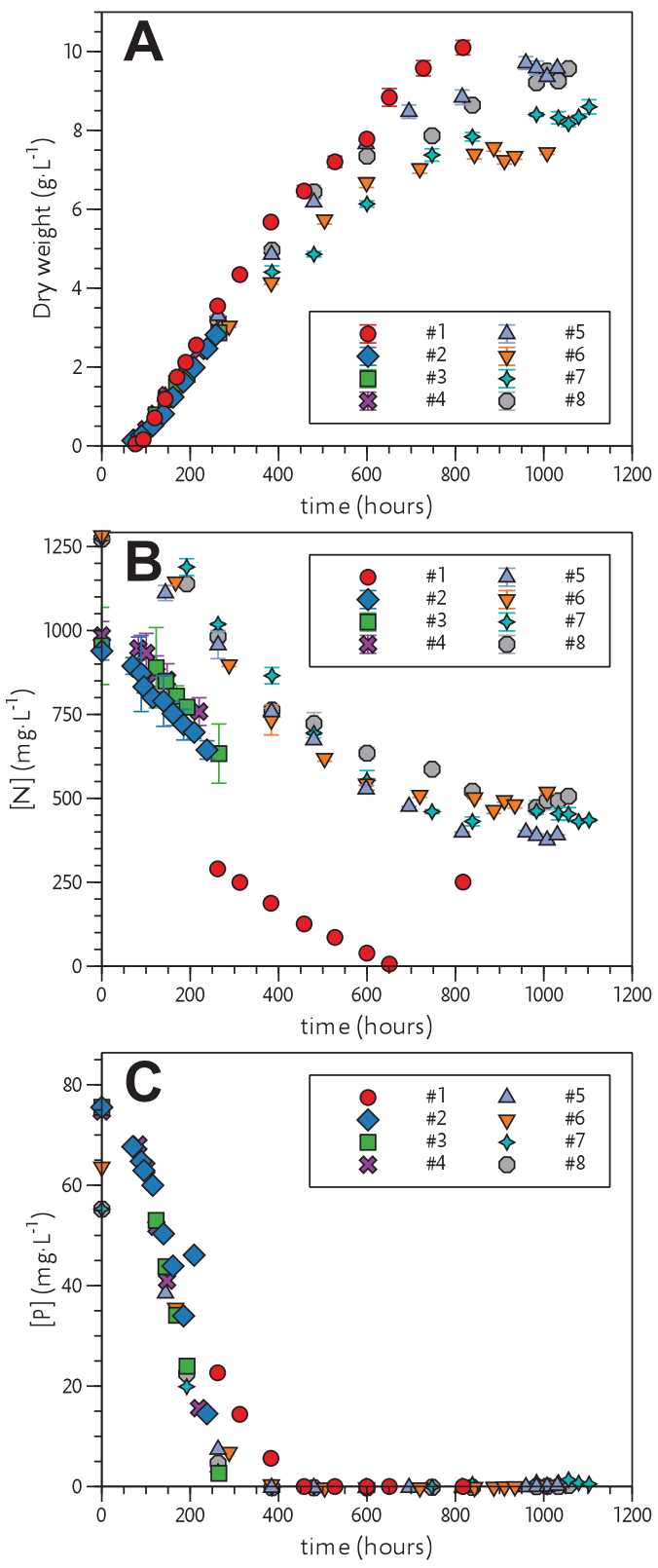

Figure 6.1. Dry weight of 8 independent batch runs of Tetradesmus obliquus (A). Supernatant nitrogen (B) and phosphorous (C) concentration during the eight different batch runs. Error bars in the dry weight represent standard deviation for triplicate measurements. Error bars in nitrogen measurements represent the deviation in technical replicates $(n \geq 2)$. 
To verify that the cultures were light-limited and that nitrogen and phosphorus concentration were not limiting growth, we measured the nitrogen and phosphate concentration in the supernatants (Figure 6.1B and C). Nitrogen and phosphorus concentration decreased throughout the cultivation. For replicate \#1 data is available from $260 \mathrm{~h}$ onwards. In this run, nitrogen limitation was observed and $\mathrm{KNO}_{3}$ was added again. To prevent nitrogen limitation in the following batch runs, a higher initial $\mathrm{KNO}_{3}$ concentration was used. In these runs nitrogen concentration remained above zero in the supernatant, showing that nitrogen was not limiting growth.

The phosphorus consumption was fast during all cultivations and phosphorus was depleted after approximately $300 \mathrm{~h}$. This does not necessarily mean that phosphorus was limiting growth as microalgae under certain conditions can consume more phosphorus than needed for growth (known as "luxury uptake") and store it in the form of polyphosphate (Powell et al., 2011). The average phosphorus content in freshwater algae is usually about $0.01 \mathrm{~g} \mathrm{gDw}_{\mathrm{DW}}^{-1}$ (Borchardt and Azad, 1968), and a value higher than that indicates luxury uptake. A minimum value of $0.004 \mathrm{~g} \cdot \mathrm{g}_{\mathrm{DW}}{ }^{-1}$ has been found without being qualified as harmful (Powell et al., 2008). For the different replicates, values between 0.06 and $0.006 \mathrm{~g} \cdot \mathrm{gDw}^{-1}$ were found throughout the culture (Supplementary Figure S6.1). Results therefore suggest that nitrogen and phosphorous were not limiting growth during the batch cultures.

With the biomass concentration and the absorbed photon flux density known, the light absorption rate $\mathrm{r}_{\mathrm{Ex}}$ (in $\mathrm{mmol}_{\mathrm{ph}} \cdot \mathrm{g}_{\mathrm{DW}}{ }^{-1} \cdot \mathrm{h}^{-1}$ ) was calculated for each specific growth rate $\mu$ (in $\mathrm{h}^{-1}$ ) as explained in section 6.2.5. In Figure 6.2 the specific light absorption rate is plotted as a function of the specific growth rate. Regression of the data gives a $m_{E}$ of $1.18 \pm 0.29 \mathrm{mmol} \mathrm{ph}_{\mathrm{D}} \cdot \mathrm{g}_{\mathrm{DW}}{ }^{-1} \mathrm{~h}^{-1}$ and a biomass yield on light $\mathrm{Y}_{\mathrm{x} / \mathrm{ph}}$ of $1.14 \pm 0.04 \mathrm{~g}_{\mathrm{DW}} \cdot \mathrm{mol}_{\mathrm{ph}}{ }^{-1}$. Four points (circled values in Figure 6.2) clearly deviate from the trend line. This was due to a low biomass concentration and thus a low amount of biomass in the sample, resulting in large errors in the measurement. The points were therefore not included in the regression and larger samples were taken for the subsequent cultivations. 


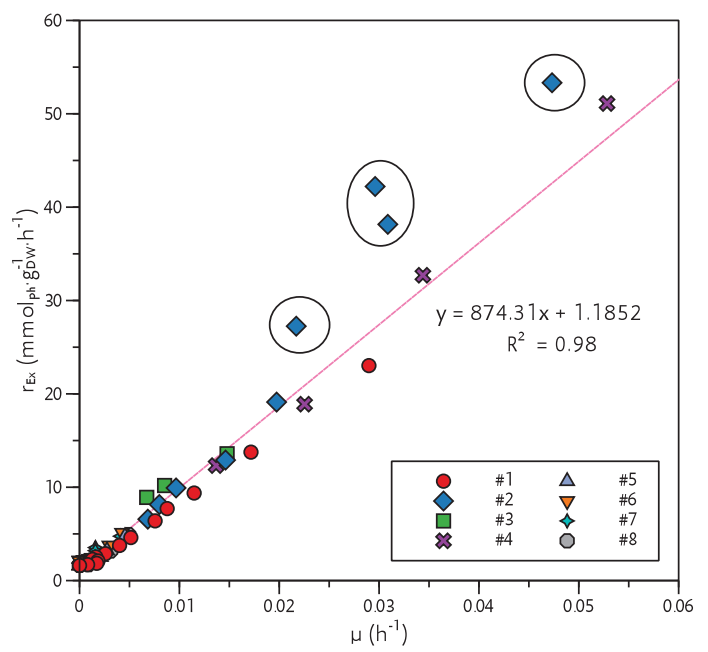

Figure 6.2. Plot of the light supply rate $r_{\mathrm{Ex}}$ against the specific growth rate calculated on different time points $(\mu)$ of eight batch runs of Tetradesmus obliquus. Circled values were not considered for the linear regression for reasons described in the main text. The $95 \%$ confidence intervals of the slope $\left(1 / Y_{x / p h}\right)$ ranged from 846.66 to 901.97 and of the intercept $\left(m_{E}\right)$ ranged from 0.89 to 1.48 .

An overview of the experimental values found with other microalgae for the biomass yield on light $\mathrm{Y}_{\mathrm{x} / \mathrm{ph}}$ (in $\mathrm{g} \cdot \mathrm{mol}_{\mathrm{ph}}{ }^{-1}$ ) and the photon maintenance requirement $\mathrm{m}_{\mathrm{E}}$ (in $\left.\mathrm{mmol} \mathrm{ph} \cdot \mathrm{gDw}^{-1} \cdot \mathrm{h}^{-1}\right)$ is presented in Table 6.2. For T. obliquus, Breuer et al. (2015b) found a value of $1.19 \pm 0.03 \mathrm{~g} \cdot \mathrm{molph}^{-1}$ under low light conditions $\left(200 \mu \mathrm{mol} \cdot \mathrm{m}^{-2} \cdot \mathrm{s}^{-1}\right)$, a value similar to the one obtained in this work. This yield was calculated from continuous chemostat cultivations based on the dilution rate, biomass concentration, light intensity and reactor thickness. The value found for the biomass yield on light was similar to that found for Chlamydomonas reinhardtii under similar low light intensity $\left(80 \mu \mathrm{mol} \cdot \mathrm{m}^{-2} \cdot \mathrm{s}^{-1}\right)$, with a biomass yield on light of $1.25 \pm 0.06 \mathrm{~g} \cdot \mathrm{mol}_{\mathrm{ph}}{ }^{-1}$ (Kliphuis et al., 2012). Under low light conditions, dissipation of light is reduced and the biomass yield on light is expected to be higher. Surprisingly, Sforza et al. (2015b) reported a value of more than twice the one obtained in this work $\left(2.52 \mathrm{~g} \cdot \mathrm{mol}_{\mathrm{ph}}{ }^{-1}\right)$ under a light intensity of $150 \mu \mathrm{mol} \cdot \mathrm{m}^{-2} \cdot \mathrm{s}^{-1} \mathrm{Th}$ is last value is high, as values for biomass yield on light are usually between 0.5 and $1.2 \mathrm{~g} \cdot \mathrm{mol}_{\mathrm{ph}}{ }^{-1}$ (Table 6.2). Under high light conditions, as expected, the values are lower as energy is dissipated or the photosystems can be damaged. For example, Breuer et al. (2015b) reported a $Y_{x / p h}$ of $0.65 \pm 0.03 \mathrm{~g} \cdot \mathrm{mol}_{\mathrm{ph}}{ }^{-1}$ at a light intensity 
of $1000 \mu \mathrm{mol} \cdot \mathrm{m}^{-2} \cdot \mathrm{s}^{-1}$; while León-Saiki et al. (2017) reported a value of $0.98 \pm 0.08 \mathrm{~g} \cdot \mathrm{molph}^{-1}$ under light conditions of $500 \mu \mathrm{mol} \cdot \mathrm{m}^{-2} \cdot \mathrm{s}^{-1}$. Overall, the biomass yield on light found for $T$. obliquus in this work is within the normal values reported in the literature under low light conditions.

Table 6.2. Energy parameters estimated for different microalgae

\begin{tabular}{|c|c|c|c|c|c|c|}
\hline Organism & $\begin{array}{c}\text { Light } \\
\text { intensity } \\
\left(\mu \mathrm{mol} \cdot \mathrm{m}^{-2} \text {. }\right. \\
\left.\mathrm{s}^{-1}\right)\end{array}$ & $\begin{array}{c}\mathrm{K}_{\mathrm{x}} \\
\underset{\left(\mathrm{mmol}_{\mathrm{ATP}}\right.}{\left.\mathrm{gDw}^{-1}\right)}\end{array}$ & $\begin{array}{c}\mathrm{m}_{\mathrm{ATP}} \\
\left(\mathrm{mmol}_{\mathrm{ATP}} \cdot\right. \\
\left.\mathrm{gDw}^{-1} \cdot \mathrm{h}^{-1}\right)\end{array}$ & $\begin{array}{c}\mathrm{Y}_{\mathrm{x} / \mathrm{ph}} \\
\left(\mathrm{g} \cdot \mathrm{mol}_{\mathrm{ph}}\right. \\
\left.{ }^{1}\right)\end{array}$ & $\begin{array}{c}\mathrm{m}_{\mathrm{E}} \\
\left(\mathrm{mmol}_{\mathrm{ph}} \cdot\right. \\
\left.\mathrm{gDw}^{-1} \cdot \mathrm{h}^{-1}\right)\end{array}$ & Reference \\
\hline $\begin{array}{l}\text { Tetradesmus } \\
\text { obliquus }^{\mathrm{a}}\end{array}$ & 90 & 121.02 & 0.66 & 1.14 & 1.18 & This work \\
\hline T. obliquus ${ }^{\text {a }}$ & 150 & - & - & 2.52 & 3.25 & $\begin{array}{c}\text { Sforza et al. } \\
\text { (2015b) }\end{array}$ \\
\hline T. obliquus ${ }^{\text {a }}$ & 650 & - & - & 1.13 & 19.65 & $\begin{array}{c}\text { Sforza et al. } \\
\text { (2015b) }\end{array}$ \\
\hline $\begin{array}{l}\text { Chlamydomonas } \\
\text { reinhardtii }{ }^{\text {b }}\end{array}$ & 35 & - & 1.68 & - & - & $\begin{array}{c}\text { Chen and } \\
\text { Johns (1996) }\end{array}$ \\
\hline C. reinhardtii ${ }^{\text {a }}$ & 65 & $29.89^{b}$ & $1.50^{c}$ & 5.31 & - & $\begin{array}{c}\text { Boyle and } \\
\text { Morgan } \\
(2009)\end{array}$ \\
\hline C. reinhardtii ${ }^{\text {a }}$ & $<100$ & 109.00 & 2.85 & 1.25 & 5.98 & $\begin{array}{c}\text { Kliphuis et al. } \\
\text { (2012) }\end{array}$ \\
\hline C. reinhardtii ${ }^{\text {a }}$ & 150 & 92.43 & 2.85 & - & - & $\begin{array}{l}\text { Imam et al. } \\
(2015)\end{array}$ \\
\hline $\begin{array}{l}\text { Chlorella sorokiniana } \\
\text { a }\end{array}$ & 930 & - & - & 0.75 & 6.80 & $\begin{array}{l}\text { Zijffers et al. } \\
\text { (2010) }\end{array}$ \\
\hline$\underset{\mathrm{a}}{\text { Dunaliella tertiolecta }}$ & 930 & - & - & 0.78 & 13.30 & $\begin{array}{l}\text { Zijffers et al. } \\
\text { (2010) }\end{array}$ \\
\hline $\begin{array}{l}\text { Nannochloropsis } \\
\text { salina }^{\text {a }}\end{array}$ & $150-600$ & - & - & 1.68 & 7.62 & $\begin{array}{l}\text { Sforza et al. } \\
\text { (2015a) }\end{array}$ \\
\hline
\end{tabular}

$\mathrm{K}_{\mathrm{x}}$ represents the ATP requirement for the formation of biomass from biopolymers, mATP is the maintenance requirement, $\mathrm{Y}_{\mathrm{x} / \mathrm{ph}}$ is the biomass yield on light and $\mathrm{m}_{\mathrm{E}}$ is the photon maintenance energy.

a phototrophic growth on light and $\mathrm{CO}_{2}$.

${ }^{b}$ heterotrophic growth on acetate. Acetate yields 9 ATP (Berg et al., 2002).

${ }^{\mathrm{c}}$ Not measured, taken from literature.

The photon maintenance requirement $\left(m_{E}\right)$ obtained from the slope (1.18 $\left.\mathrm{mmol} \mathrm{ph} \cdot \mathrm{g}_{\mathrm{DW}}{ }^{-1} \cdot \mathrm{h}^{-1}\right)$ is one of the lowest value reported for microalgae using this method (Table 6.2). When comparing the value obtained to the one reported by Sforza et al. (2015b) under low light $\left(150 \mu \mathrm{mol} \cdot \mathrm{m}^{-2} \cdot \mathrm{s}^{-1}\right)$ for $T$. obliquus, the value is more than double. The difference could be explained by dissipation or photoinhibition (as the light intensity used was above the saturation value of $89 \mu \mathrm{mol} \cdot \mathrm{m}^{-2} \cdot \mathrm{s}^{-1}$ estimated based on Breuer et al., (2015a)). The other $m_{E}$ values found for algae (Table 6.2), with the exception of the one presented by Kliphuis et al. (2012), were calculated under high 
light intensity, where maintenance requirements have been reported to increase at higher irradiances, probably related to photoinhibition and saturation (Sforza et al., 2015b) Additionally, the photon maintenance value was calculated from the stationary phase, where growth is zero, and we assume all the photons are used for maintenance. Using this second approach, the value was found to be $1.80 \pm 0.19 \mathrm{mmol}_{\mathrm{ph}} \cdot \mathrm{gDw}_{\mathrm{DW}}{ }^{-1} \cdot \mathrm{h}^{-1}$. This higher value may be caused by additional stress experienced by the algae during the stationary phase.

The metabolic network always contains a reaction which describes the synthesis of biomass from macromolecules (biomass equation). The formulation of this equation strongly depends on the biomass composition and the energetic requirements to generate new biomass (Feist and Palsson, 2010). To calculate the biomass equation coefficients, the biomass composition was analysed for the specific growth rates calculated on different time points (Figure 6.3). Protein content was the major component of the dry weight, ranging from 0.40 to $0.80 \mathrm{~g} \cdot \mathrm{g}_{\mathrm{DW}}{ }^{-1}$ (Figure 6.3B). Followed by carbohydrates (approximate $0.10-0.20 \mathrm{~g} \cdot \mathrm{g}_{\mathrm{DW}}{ }^{-1}$, Figure $6.3 \mathrm{~A}$ ) and polar lipids (approximately $0.08 \mathrm{~g} \cdot \mathrm{g}_{\mathrm{DW}}{ }^{-1}$, Figure $6.3 \mathrm{C}$ ).

In general, the biomass composition remained more or less constant, except at low growth rates below $0.01 \mathrm{~h}^{-1}$, where the carbohydrate content increased (Figure 6.3A), and protein (Figure 6.3B) content decreased. The chlorophyll content was stable around $0.03 \mathrm{~g} \cdot \mathrm{gDw}^{-1}$, (Figure 6.3E) and tryacylglycerols content and carotene content remained stable and close to zero (Figure 6.3D and F). With the aforementioned measured biomass components (together with the ash content), we could explain in average $90 \%$ of the biomass dry weight. Assuming that DNA and RNA content together account for $6.2 \%$ of the biomass, the mass balance for the biomass components was between $90-100 \%$, which is satisfactory for the modelling approach.

Using the biomass composition, the biomass coefficients were calculated as explained in section 6.2.8 and values are presented in the Supplementary Table S6.1. These values were then incorporated into the biomass synthesis reaction (reaction 220, 'BIOMASS_S', Supplementary File S6.1) to estimate the ATP production rate. 

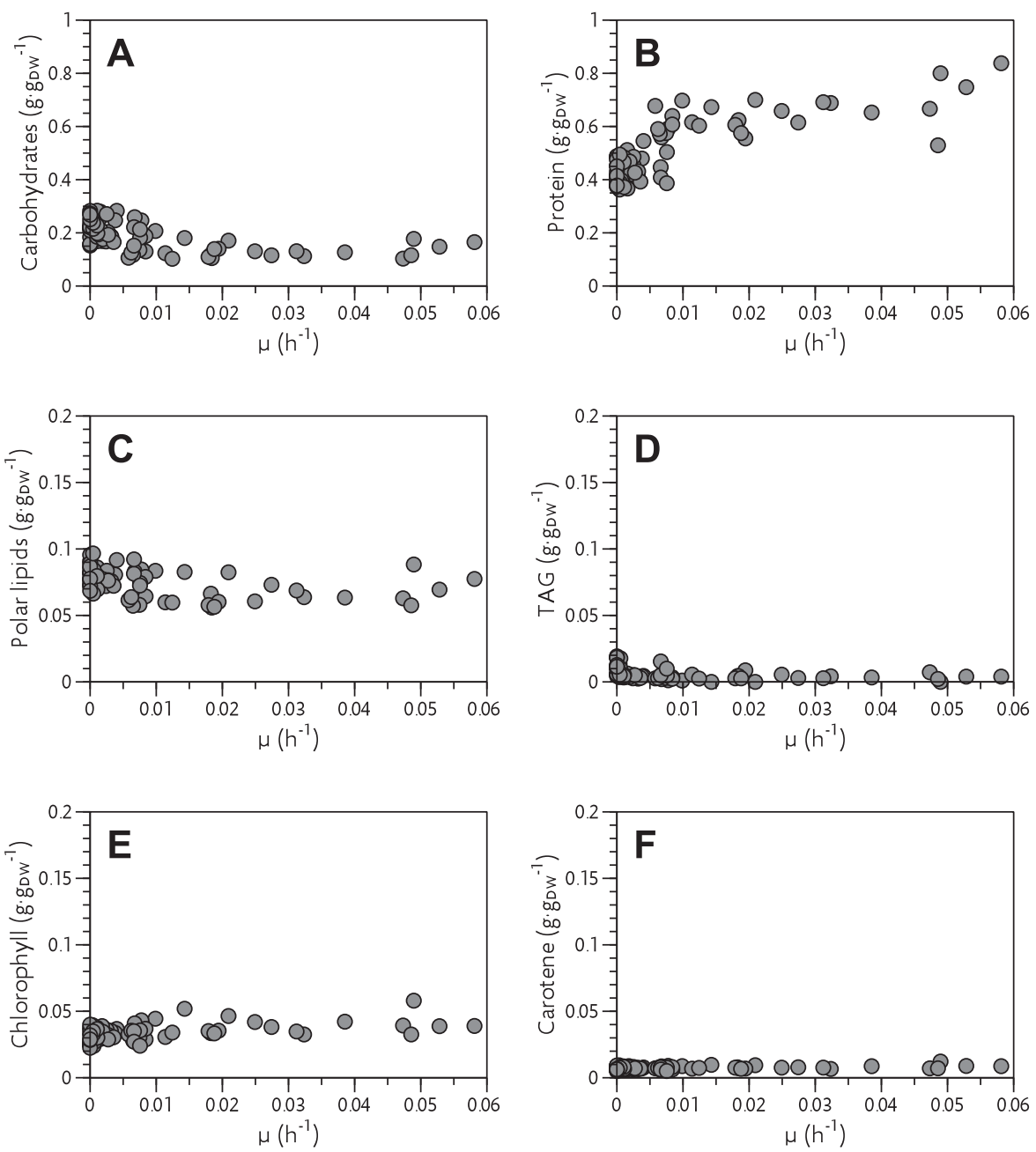

Figure 6.3. Measured biomass composition under different growth rates for Tetradesmus obliquus. A) carbohydrates; B) proteins; C) polar lipids; D) triacylglycerol (TAG); E) chlorophyll; and F) carotene content.

\subsubsection{Estimation of energy requirements for growth and maintenance}

Using the biomass coefficients (Supplementary Table S6.1), the specific light absorption rates and the specific growth rates $\mu$ as input for the model, the qATP was estimated for each $\mu$, and results are shown in Figure 6.4. According to Eq. 6.2, the slope of this line gives the $K_{x}$, the ATP requirement to make biomass from biopolymers, 
and has a value of $121.02 \mathrm{mmol}_{\mathrm{ATP}} \cdot \mathrm{g}_{\mathrm{DW}}{ }^{-1}$ (95\% confidence interval 106.14 to $\left.135.91 \mathrm{mmol}_{\mathrm{ATP}} \cdot \mathrm{g}_{\mathrm{DW}}{ }^{-1}\right)$. The intercept of this line represents the ATP required for maintenance ( $\mathrm{m}_{\text {ATP }}$ ) based on the model, which has a value of $0.66 \mathrm{mmol}_{\text {ATP }} \cdot \mathrm{gDW}_{\mathrm{DW}}{ }^{-1} \cdot \mathrm{h}^{-1}$ $\left(95 \%\right.$ confidence interval 0.50 to $\left.0.82 \mathrm{mmol}_{A T P} \cdot \mathrm{gDW}^{-1} \cdot \mathrm{h}^{-1}\right)$. As for the photon maintenance $\left(\mathrm{m}_{\mathrm{E}}\right)$, the ATP maintenance value was calculated from the stationary phase. Using this approach, the value was found to be $0.97 \pm 0.10 \mathrm{mmol}_{A T P} \mathrm{~g}_{D W^{-1}} \mathrm{~h}^{-1}$. This is higher than the value obtained from the regression $(p<0.05)$, which, as for $m_{E}$, may be due to additional stress experienced by the algae during the stationary phase.

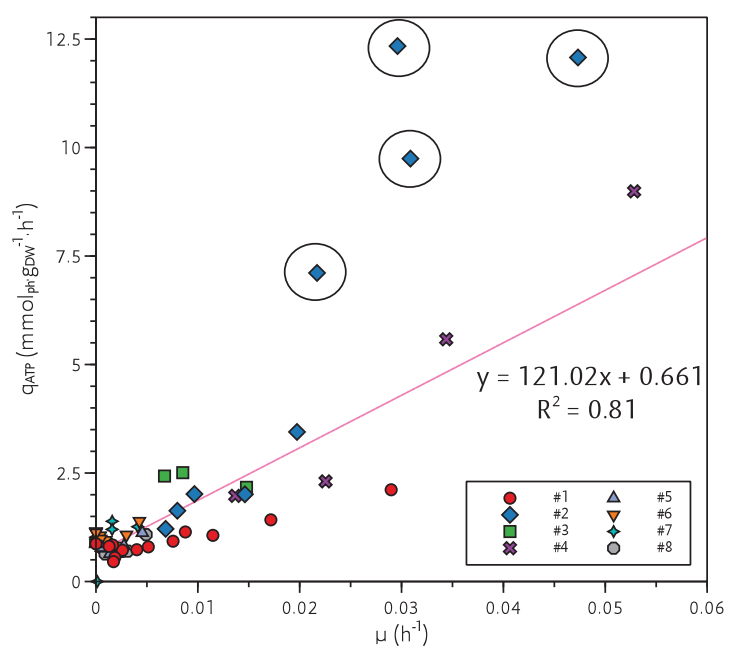

Figure 6.4. Calculated specific overall ATP production rate $\left(q_{A T P}\right.$ in $\left.\mathrm{mmol} \cdot \mathrm{g}_{D W^{-1}} \cdot \mathrm{h}^{-1}\right)$ against the specific growth rate calculated on different time points $\left(\mu\right.$ in $\left.h^{-1}\right)$ for Tetradesmus obliquus. $\mathrm{q}_{\text {ATP }}$ was calculated using the model for each growth rate and corresponding biomass equation. Circled values were not considered for the linear regression for reasons described in section 6.3.2.

The estimation of energy parameters has been done in organisms ranging from Escherichia coli (Kayser et al., 2005), Saccharomyces cerevisiae (Förster et al., 2003), Lactococcus lactis (Oliveira et al., 2005), and archae (Thor et al., 2017), to microalgae (Boyle and Morgan, 2009; Kliphuis et al., 2012; Sforza et al., 2015b). We presented in Table 6.2 an overview of the values for $K_{x}\left(\right.$ in $\mathrm{mmol}_{\text {ATP }} \cdot \mathrm{g}_{\mathrm{DW}}{ }^{-1}$ ), and $\mathrm{m}_{\text {ATP }}$ (in mmol $\mathrm{mTP}$. $\left.\mathrm{gDw}^{-1} \cdot \mathrm{h}^{-1}\right)$ found in literature for different microalgae. 
The variation in the values for $K_{x}$ can be explained by differences in model used, conditions applied, and differences between species (Kliphuis et al., 2012). Regarding $\mathrm{K}_{\mathrm{x}}$, the value for $T$. obliquus was comparable to that of $C$. reinhardtii reported by Kliphuis et al. (2012). A much lower value of $29.89 \mathrm{mmol}_{\mathrm{ATP}} \cdot \mathrm{g}_{\mathrm{DW}}{ }^{-1}$ was previously reported for C. reinhardtii (Boyle and Morgan, 2009). The model used by Boyle and Morgan (2009) was similar to the one developed for T. obliquus (484 metabolic reactions and 458 metabolites divided between the chloroplast, mitochondria, cytosol and extracellular space). However, their value of $\mathrm{K}_{\mathrm{x}}$ was calculated under heterotrophic conditions (on acetate), and assumed to remain equal under autotrophic conditions. However, by calculating the biomass on light yield based on their results, a value that is 4 times as high as our value is obtained ( $28.9 \mathrm{~g}$ per mole of carbon using 544 moles of photons per 100 moles of $\mathrm{CO}_{2}$, resulting in $5.3 \mathrm{~g} \cdot \mathrm{mol}_{\mathrm{ph}}{ }^{-1}$ ). This is unlikely and thus the growth associated maintenance is likely higher under photo-autotrophic conditions than under heterotrophic growth conditions.

Many studies have focused on estimating the maintenance energy requirement of the cells ( $\left.m_{\text {ATP }}\right)$. The estimation of the maintenance energy is needed to understand the energy requirements for the cells to grow (Sforza et al., 2015b), especially at low growth rates. For high growth rates the maintenance energy is usually negligible. The maintenance value of $0.66 \mathrm{mmol}_{\mathrm{ATP}} \cdot \mathrm{g}_{\mathrm{DW}}{ }^{-1} \cdot \mathrm{h}^{-1}$ found for $T$. obliquus based on the model is, to our knowledge, one of the lowest reported in literature for microalgae (Table 6.2), and other organisms such as E. coli (2.81 $\left.\mathrm{mmol}_{\text {ATP }} \cdot \mathrm{gDW}^{-1} \cdot \mathrm{h}^{-1}\right)$ (Kayser et al., 2005), Aspergillus niger $\left(3.73 \mathrm{mmol}_{\mathrm{ATP}} \cdot \mathrm{g}_{\mathrm{DW}}{ }^{-1} \cdot \mathrm{h}^{-1}\right)$ (Lu et al., 2017), and S. cerevisiae $\left(1.00 \mathrm{mmol}_{\mathrm{ATP}} \cdot \mathrm{g}_{\mathrm{DW}}{ }^{-1} \cdot \mathrm{h}^{-1}\right)$ (Förster et al., 2003). As expected, these low values are in agreement with the low value of maintenance in photons $\left(m_{E}\right)$ found (Table 6.2). This low values cannot be explained by the simplification of the model (primary metabolism), as the maintenance energy is large determined by the amount of absorbed light and the conversion efficiency to ATP in the absence of growth. Reactions involved on this are only a small part of the models. In this model, we assumed the practical maximum conversion efficiency, which is very comparable to other models. An extra indication of the low maintenance requirement in T. obliquus, was its capacity to grow for over 40 days with a low amount of light $\left(90 \mu \mathrm{mol} \cdot \mathrm{m}^{-2} \cdot \mathrm{s}^{-1}\right)$. 


\subsubsection{Theoretical yields}

Next, the model output was evaluated. For this, the value of $\mathrm{K}_{\mathrm{x}}$ was incorporated into the biomass synthesis reaction (in mol $\mathrm{ATP} \cdot \mathrm{gDW}^{-1}$, reaction 220 ) of the final model. The biomass equation (in $\mathrm{mmol} \cdot \mathrm{gDW}_{\mathrm{DW}}{ }^{-1}$ ) was:

$$
\begin{gathered}
\text { 0.7887Carbohydrate }+0.0849 \text { MGDG }+5.8576 \text { Protein }+0.0433 \text { Chlorophyll }+ \\
0.0141 \text { Carotene }+0.1769 R N A+0.0056 D N A+121.02 A T P+121.02 \mathrm{H}_{2} \mathrm{O} \rightarrow \\
\text { Biomass }+121.02 \mathrm{ADP}+121.02 \mathrm{P}_{i}+121.02 \mathrm{H}^{+}
\end{gathered}
$$

Where MGDG stands for monogalactosyl-diacylglycerol, main component of membrane lipids (polar lipids). The yields of biomass, starch, and TAG on light (in $\mathrm{g} \cdot \mathrm{mol}_{\mathrm{ph}}{ }^{-1}$ ) were calculated based on the model using geometric Flux Balance Analysis (FBA) and results are shown in Table 6.3.

Table 6.3. Theoretical maximum yields

\begin{tabular}{lccccc}
\hline Organism & $\begin{array}{c}\mathbf{Y}_{\mathrm{x} / \mathrm{ph}} \\
\left(\mathbf{g}_{\mathrm{DW}} \cdot \mathbf{m o l}_{\mathrm{ph}}{ }^{-1}\right)\end{array}$ & $\begin{array}{c}\mathbf{Y}_{\mathrm{TAG} / \mathrm{ph}} \\
\left(\mathbf{g}_{\mathrm{TAG}} \cdot \mathbf{m o l}_{\mathrm{ph}}{ }^{-1}\right)\end{array}$ & $\begin{array}{c}\mathbf{Y}_{\mathrm{starch} / \mathrm{ph}} \\
\left(\mathbf{g}_{\mathrm{starch}} \cdot \mathbf{m o l}_{\mathrm{ph}}{ }^{-1}\right)\end{array}$ & $\begin{array}{c}\mathbf{Y}_{\mathrm{TAG} / \mathrm{Starch}} \\
\left(\mathbf{g}_{\mathrm{TAG}} \cdot \mathbf{g}_{\mathrm{starch}}{ }^{-1}\right)\end{array}$ & Reference \\
\hline T. obliquus & 1.15 & 1.05 & 2.69 & 0.38 & This work \\
T. obliquus & 1.62 & 1.33 & 3.24 & 0.39 & Breuer et al. (2015a) \\
\hline
\end{tabular}

As expected, the theoretical biomass yield on light was the same as the one calculated from the experimental values (Table 6.2), but this was lower than the maximum value estimated by Breuer et al. (2015a) (Table 6.3). The TAG yield on light of T. obliquus is in the same range as the one estimated by Imam et al. (2015) in the metabolic model iCre1355 of Chlamydomonas reinhardtii $\left(1.43 \mathrm{~g}_{\mathrm{TAG}} \cdot \mathrm{mol}_{\mathrm{ph}}{ }^{-1}\right)$. The starch yield on light and the TAG yield on light estimated by Breuer et al. (2015a) are again higher than the values predicted by the model presented in this work. The differences observed between the predictions of this model and the one developed by Breuer et al. (2015a) can be attributed to differences in the equation for the light reaction. Breuer et al. (2015a) assumed that 8 photons produce 3 ATP and 2 NADPH during the linear electron transport, while we assumed that 10 photons were needed to give the same outcome. This would reduce the values of Breuer et al. (2015a) by $20 \%$ and would then become similar to those predicted by our model. 
Overall, these values are the theoretical maximum based on the model, without considering maintenance energy and assuming that only the desired product is formed (e.g. for biomass, no TAG nor starch is formed).

\subsection{Conclusions}

We constructed a metabolic model describing the primary metabolism of Tetradesmus obliquus. The energy parameters were estimated from a set of batch-operated bioreactor runs under low light conditions, resulting in an ATP requirement to make biomass from biopolymers $\left(K_{x}\right)$ of $121.02 \mathrm{mmol}_{\text {ATP }} \cdot \mathrm{g}_{D W^{-1}}$ and an ATP requirement for maintenance $\left(\mathrm{m}_{\text {ATP }}\right.$ ) of $0.66 \mathrm{mmol}_{\text {ATP }} \cdot \mathrm{g}_{\mathrm{DW}}{ }^{-1} \cdot \mathrm{h}^{-1}$. The prediction of the model on biomass yield on light $\left(1.15 \mathrm{~g}_{\mathrm{DW}} \cdot \mathrm{mol}_{\mathrm{ph}}{ }^{-1}\right)$, TAG yield on light $\left(1.05 \mathrm{~g}_{\mathrm{TAG}} \cdot \mathrm{mol}_{\mathrm{ph}}{ }^{-1}\right)$, starch yield on light $\left(2.69 \mathrm{~g}_{\text {starch }} \cdot \mathrm{mol}_{\mathrm{ph}}{ }^{-1}\right)$ and TAG yield on starch $\left(0.38 \mathrm{~g}_{\text {TAG }} \cdot \mathrm{g}_{\mathrm{starch}}{ }^{-1}\right)$ are within the range of values reported in the literature.

In addition, as the model describes the primary metabolism, customization towards other microalgae would be feasible by changing the biomass synthesis equation, estimating the corresponding energy parameters, and including some extra algae specific pathways if necessary, such as hydrogen production or fermentation from other carbon sources.

\section{Funding}

This research project was supported by the Consejo Nacional de Ciencia y Tecnología - CONACYT, Mexico, Scholar 218586/Scholarship 314173. In addition, GMLS is part of the program "Doctores Jóvenes para el Desarrollo Estratégico Institucional” by the Universidad Autónoma de Sinaloa.

\section{Acknowledgments}

The authors would like to thank Wendy Evers for her help with the biochemical analysis. 


\section{Supplementary information}

\section{Supplementary File S6.1. Reaction equations}

Arrows indicate the direction an reversibility of the reactions. Letter between brackets ' []$^{\prime}$ indicate the compartment, where ' $h$ ' stands for chloroplast, ' $c$ ' for cytosol, ' $m$ ' for mitochondria, and ' $\mathrm{e}$ ' extracellular space. First column includes the reaction number. Second column indicate the E.C. number. E.C. numbers separated by "," indicate "and", and separated by "I" indicate "or".

\section{Photosynthesis}

1

2

$\begin{array}{ll}2 & \\ 3 & 3.6 .3 .14\end{array}$
$3 \mathrm{ADP}[\mathrm{h}]+\mathrm{H}[\mathrm{h}]+2 \mathrm{NADP}[\mathrm{h}]+3 \mathrm{Pi}[\mathrm{h}]+10$ photon[h] $->3 \mathrm{ATP}[\mathrm{h}]+\mathrm{H} 2 \mathrm{O}[\mathrm{h}]+2$ $\mathrm{NADPH}[\mathrm{h}]+\mathrm{O} 2[\mathrm{~h}]$

$\mathrm{ADP}[\mathrm{h}]+\mathrm{H}[\mathrm{h}]+\mathrm{Pi}[\mathrm{h}]+2$ photon $[\mathrm{h}]->\mathrm{ATP}[\mathrm{h}]+\mathrm{H} 2 \mathrm{O}[\mathrm{h}]$

$\mathrm{ATP}[\mathrm{h}]+\mathrm{H} 2 \mathrm{O}[\mathrm{h}]->\mathrm{ADP}[\mathrm{h}]+\mathrm{H}[\mathrm{h}]+\mathrm{Pi}[\mathrm{h}]$

photon[h] $\rightarrow$ fluores[h]

\section{Oxidative phosphorylation}

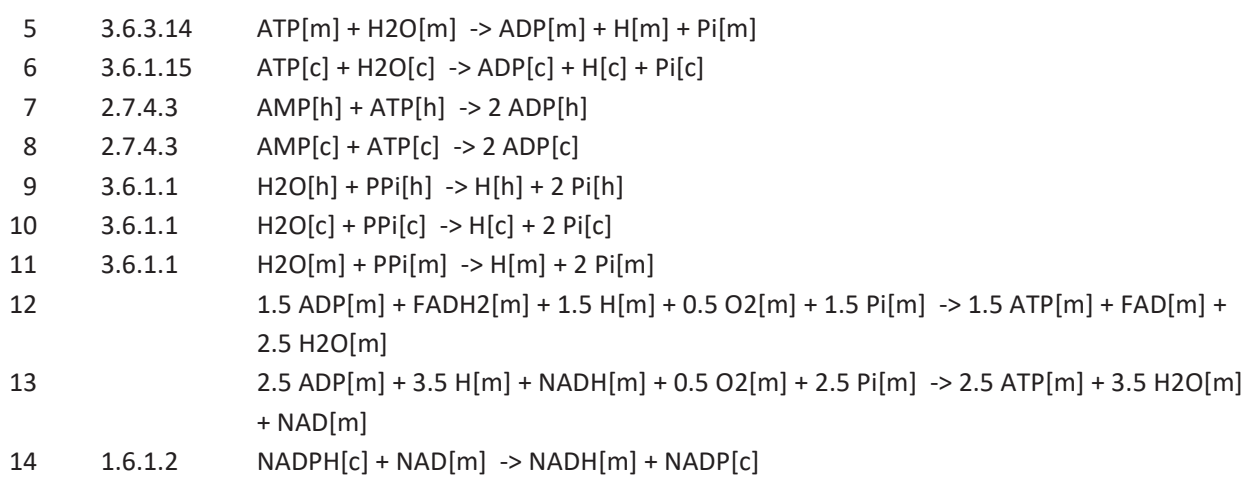

\section{Calvin cycle}

\begin{tabular}{|c|c|c|}
\hline 15 & 4.1.1.39 & $\mathrm{CO} 2[\mathrm{~h}]+\mathrm{H} 2 \mathrm{O}[\mathrm{h}]+\mathrm{RU} 15 \mathrm{DP}[\mathrm{h}] \quad \rightarrow 23 \mathrm{PG}[\mathrm{h}]$ \\
\hline 16 & 2.7.2.3 & $3 P G[h]+A T P[h]->13 D P G[h]+A D P[h]+H[h]$ \\
\hline 17 & 1.2.1.13 & $13 D P G[h]+H[h]+N A D P H[h] \Leftrightarrow=\operatorname{GAP}[h]+N A D P[h]+P i[h]$ \\
\hline 18 & 5.3.1.1 & GAP $[h] \Leftrightarrow=>$ DHAP $[h]$ \\
\hline 19 & 4.1.2.13 & $\mathrm{DHAP}[\mathrm{h}]+\mathrm{GAP}[\mathrm{h}] \Leftrightarrow=>\mathrm{F} 16 \mathrm{P}[\mathrm{h}]$ \\
\hline 20 & 3.1.3.11 & $\mathrm{F} 16 \mathrm{P}[\mathrm{h}]+\mathrm{H} 2 \mathrm{O}[\mathrm{h}]->\mathrm{F} 6 \mathrm{P}[\mathrm{h}]+\mathrm{Pi}[\mathrm{h}]$ \\
\hline 21 & 2.2.1.1 & $F 6 P[h]+G A P[h]<=>E 4 P[h]+X 5 P[h]$ \\
\hline 22 & $\begin{array}{l}\text { 5.3.1.1, } \\
4.1 .2 .13\end{array}$ & $\mathrm{E} 4 \mathrm{P}[\mathrm{h}]+\mathrm{GAP}[\mathrm{h}]+\mathrm{H} 2 \mathrm{O}[\mathrm{h}] \Leftrightarrow=>\mathrm{Pi}[\mathrm{h}]+\mathrm{S} 7 \mathrm{P}[\mathrm{h}]$ \\
\hline 23 & 2.2.1.1 & GAP $[h]+S 7 P[h] \Leftrightarrow=>$ R5P[h] +X5P[h] \\
\hline 24 & 2.2.1.2 & GAP $[h]+S 7 P[h] \Leftrightarrow=>E 4 P[h]+F 6 P[h]$ \\
\hline 25 & 5.1.3.1 & $X 5 P[h] \Leftrightarrow=>R U 5 P[h]$ \\
\hline 26 & 5.3.1.6 & R5P[h] $<=>$ RU5P[h] \\
\hline 27 & 2.7.1.19 & $A T P[h]+R U 5 P[h]->A D P[h]+H[h]+R U 15 D P[h]$ \\
\hline
\end{tabular}


28 1.1.1.40 MAL[h] + NADP $[h] \Leftrightarrow=>\mathrm{CO} 2[h]+N A D P H[h]+P Y R[h]$

\section{Glycolysis/gluconeogenesis}

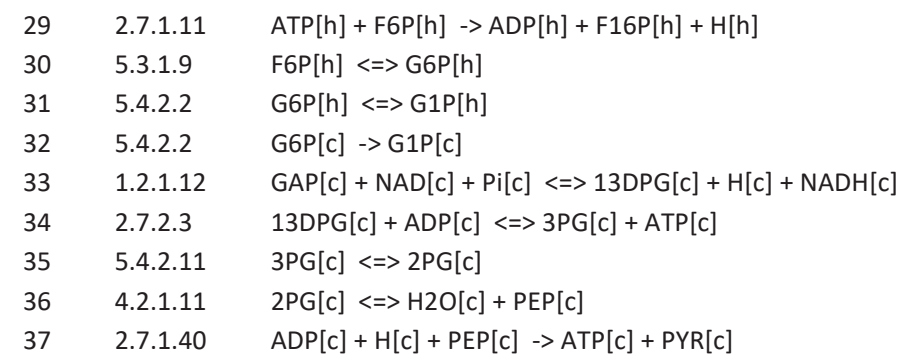

\section{Photorespiration}

\begin{tabular}{|c|c|c|}
\hline 38 & $\begin{array}{l}\text { 4.1.1.39, } \\
3.1 .3 .18\end{array}$ & $\mathrm{H} 2 \mathrm{O}[\mathrm{h}]+\mathrm{O} 2[\mathrm{~h}]+\mathrm{RU} 15 \mathrm{DP}[\mathrm{h}]->3 \mathrm{PG}[\mathrm{h}]+\mathrm{H}[\mathrm{h}]+\mathrm{Pi}[\mathrm{h}]+$ glycolate $[\mathrm{h}]$ \\
\hline 39 & 1.1.1.26/ & $\mathrm{NAD}[\mathrm{m}]+$ glycolate $[\mathrm{m}]->\mathrm{GLYX}[\mathrm{m}]+\mathrm{H}[\mathrm{m}]+\mathrm{NADH}[\mathrm{m}]$ \\
\hline & 1.1.99.14 & \\
\hline 40 & 1.1.3.15 & $\mathrm{O} 2[\mathrm{c}]+$ glycolate $[\mathrm{c}]->\mathrm{GLYX}[\mathrm{c}]+\mathrm{H} 2 \mathrm{O} 2[\mathrm{c}]$ \\
\hline 41 & 2.6.1.44 & $\mathrm{GLY}[\mathrm{m}]+\mathrm{H}[\mathrm{m}]+\operatorname{PYR}[\mathrm{m}] \Leftrightarrow=\mathrm{ALA}[\mathrm{m}]+\mathrm{GLYX}[\mathrm{m}]$ \\
\hline 42 & 2.1.2.1 & $\mathrm{GLY}[\mathrm{m}]+\mathrm{H} 2 \mathrm{O}[\mathrm{m}]+\mathrm{METHF}[\mathrm{m}]->\mathrm{SER}[\mathrm{m}]+\mathrm{THF}[\mathrm{m}]$ \\
\hline 43 & 2.6.1.45 & $\mathrm{GLYX}[\mathrm{m}]+\mathrm{SER}[\mathrm{m}]<=>\mathrm{GLY}[\mathrm{m}]+\mathrm{HPYR}[\mathrm{m}]$ \\
\hline 44 & 2.1.2.10 & $\mathrm{GLY}[\mathrm{m}]+\mathrm{NAD}[\mathrm{m}]+\mathrm{THF}[\mathrm{m}] \Leftrightarrow=\mathrm{CO} 2[\mathrm{~m}]+\mathrm{METHF}[\mathrm{m}]+\mathrm{NADH}[\mathrm{m}]+\mathrm{NH} 4[\mathrm{~m}]$ \\
\hline 45 & 1.1.1.29 & $\mathrm{HPYR}[\mathrm{m}]+\mathrm{H}[\mathrm{m}]+\mathrm{NADH}[\mathrm{m}] \Leftrightarrow=>\mathrm{GLYT}[\mathrm{m}]+\mathrm{NAD}[\mathrm{m}]$ \\
\hline 46 & 1.2.1.3 & $\mathrm{GLYT}[\mathrm{c}]+\mathrm{H}[\mathrm{c}]+\mathrm{NADH}[\mathrm{c}] \Leftrightarrow=>\mathrm{GA}[\mathrm{c}]+\mathrm{H} 2 \mathrm{O}[\mathrm{c}]+\mathrm{NAD}[\mathrm{c}]$ \\
\hline 47 & 1.1.1.2 & $\mathrm{GA}[\mathrm{c}]+\mathrm{H}[\mathrm{c}]+\mathrm{NADPH}[\mathrm{c}]<=>\mathrm{GLYC}[\mathrm{c}]+\mathrm{NADP}[\mathrm{c}]$ \\
\hline 48 & 2.7.1.30 & $\mathrm{ATP}[\mathrm{c}]+\mathrm{GLYC}[\mathrm{c}]->\mathrm{ADP}[\mathrm{c}]+\mathrm{GLYC3P}[\mathrm{c}]+\mathrm{H}[\mathrm{c}]$ \\
\hline 49 & 2.7.1.31 & $A T P[h]+G L Y T[h]->3 P G[h]+A D P[h]+2 H[h]$ \\
\hline
\end{tabular}

\section{Starch metabolism}

\begin{tabular}{|c|c|c|}
\hline 50 & 2.7.7.27 & ATP $[h]+$ G1P[h] + H[h] $->$ ADPG[h] + PPi[h] \\
\hline 51 & $\begin{array}{l}\text { 2.4.1.21, } \\
\text { 2.4.1.18 }\end{array}$ & ADPG[h] $->\mathrm{ADP}[\mathrm{h}]+\mathrm{H}[\mathrm{h}]+\mathrm{STARCH}[\mathrm{h}]$ \\
\hline 52 & & $\mathrm{ATP}[\mathrm{h}]+\mathrm{STARCH}[\mathrm{h}] \quad->\mathrm{ADP}[\mathrm{h}]+\mathrm{H}[\mathrm{h}]+\mathrm{STARCH}$ ddash_P$[\mathrm{h}]$ \\
\hline 53 & & $\mathrm{H} 2 \mathrm{O}[\mathrm{h}]+\mathrm{STARCH}$ dash_P$[\mathrm{h}]+\mathrm{STARCH}[\mathrm{h}]$-> Malt_dash_P[h] \\
\hline 54 & & Malt_dash_P $[\mathrm{c}]+\mathrm{Pi}[\mathrm{c}] \Leftrightarrow=>2 \mathrm{G} 1 \mathrm{P}[\mathrm{c}]$ \\
\hline
\end{tabular}

\section{Nitrogen assimilation}

\begin{tabular}{|c|c|}
\hline 55 & 1.7.1.2/ \\
\hline & 1.7.1.1 \\
\hline 6 & 1.7.7.1 \\
\hline 7 & 6.3.1.2 \\
\hline 58 & 1.4.7.1 \\
\hline & 1.4.1.14 \\
\hline & 1.4.1.4 \\
\hline & 1.4.1.2 \\
\hline
\end{tabular}

$\mathrm{H}[\mathrm{c}]+\mathrm{NADH}[\mathrm{c}]+\mathrm{NO} 3[\mathrm{c}] \Leftrightarrow=>\mathrm{H} 2 \mathrm{O}[\mathrm{c}]+\mathrm{NAD}[\mathrm{c}]+\mathrm{NO} 2[\mathrm{c}]$

$6 \mathrm{H}[\mathrm{h}]+\mathrm{NO} 2[\mathrm{~h}]+6 \mathrm{fdxrd}[\mathrm{h}]->2 \mathrm{H} 2 \mathrm{O}[\mathrm{h}]+\mathrm{NH} 4[\mathrm{~h}]+6 \mathrm{fdxox}[\mathrm{h}]$ $A T P[h]+G L U[h]+N H 4[h]->A D P[h]+G L N[h]+H[h]+P i[h]$ $A K G[h]+G L N[h]+H[h]+2 f d x r d[h]->2 G L U[h]+2 f d x o x[h]$ $A K G[h]+G L N[h]+H[h]+N A D H[h]->2 G L U[h]+N A D[h]$ $\mathrm{GLU}[\mathrm{h}]+\mathrm{H} 2 \mathrm{O}[\mathrm{h}]+\mathrm{NADP}[\mathrm{h}]->\mathrm{AKG}[\mathrm{h}]+\mathrm{H}[\mathrm{h}]+\mathrm{NADPH}[\mathrm{h}]+\mathrm{NH} 4[\mathrm{~h}]$ $\mathrm{GLU}[\mathrm{m}]+\mathrm{H} 2 \mathrm{O}[\mathrm{m}]+\mathrm{NAD}[\mathrm{m}]->\mathrm{AKG}[\mathrm{m}]+\mathrm{H}[\mathrm{m}]+\mathrm{NADH}[\mathrm{m}]+\mathrm{NH} 4[\mathrm{~m}]$

\section{Tricarboxylic acid cycle (TCA)}




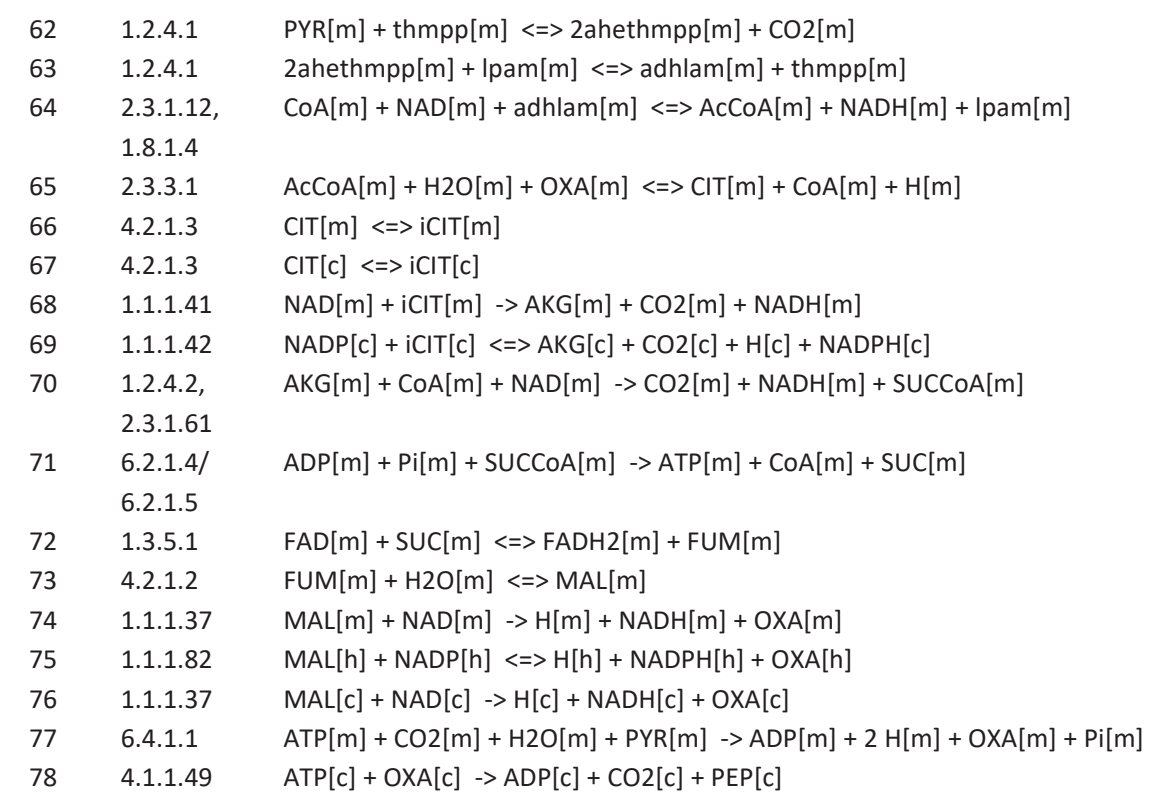

\section{Glyoxylate cycle}

$\begin{array}{lll}79 & 4.1 .3 .1 & \mathrm{iCIT}[\mathrm{m}]->\mathrm{GLYX}[\mathrm{m}]+\mathrm{SUC}[\mathrm{m}] \\ 80 & 4.1 .3 .1 & \mathrm{iCIT}[\mathrm{c}]->\mathrm{GLYX}[\mathrm{c}]+\mathrm{SUC}[\mathrm{c}] \\ 81 & 2.3 .3 .9 & \mathrm{AcCoA}[\mathrm{m}]+\mathrm{GLYX}[\mathrm{m}]+\mathrm{H} 2 \mathrm{O}[\mathrm{m}]->\mathrm{CoA}[\mathrm{m}]+\mathrm{MAL}[\mathrm{m}] \\ 82 & 2.3 .3 .9 & \mathrm{AcCoA}[\mathrm{c}]+\mathrm{GLYX}[\mathrm{c}]+\mathrm{H} 2 \mathrm{O}[\mathrm{c}]->\mathrm{CoA}[\mathrm{c}]+\mathrm{MAL}[\mathrm{c}]\end{array}$

\section{Pentose phosphate pathway}

\begin{tabular}{|c|c|c|}
\hline 83 & 1.1.1.49 & $\mathrm{G} 6 \mathrm{P}[\mathrm{c}]+\mathrm{NADP}[\mathrm{c}]->\mathrm{GLPG}[\mathrm{c}]+\mathrm{H}[\mathrm{c}]+\mathrm{NADPH}[\mathrm{c}]$ \\
\hline 84 & 1.1.1.49 & $\mathrm{G} 6 \mathrm{P}[\mathrm{h}]+\mathrm{NADP}[\mathrm{h}] \Leftrightarrow=>\mathrm{GLPG}[\mathrm{h}]+\mathrm{H}[\mathrm{h}]+\mathrm{NADPH}[\mathrm{h}]$ \\
\hline 85 & 3.1.1.31 & $\mathrm{GLPG}[\mathrm{h}]+\mathrm{H} 2 \mathrm{O}[\mathrm{h}] \quad \Leftrightarrow=>\mathrm{PG}[\mathrm{h}]+\mathrm{H}[\mathrm{h}]$ \\
\hline 86 & & $\mathrm{GLPG}[\mathrm{c}]+\mathrm{H} 2 \mathrm{O}[\mathrm{c}] \Leftrightarrow=>\mathrm{PG}[\mathrm{c}]+\mathrm{H}[\mathrm{c}]$ \\
\hline 87 & 1.1.1.44 & $6 P G[c]+N A D P[c]->C O 2[c]+N A D P H[c]+R U 5 P[c]$ \\
\hline 88 & 1.1.1.44 & $6 P G[h]+N A D P[h]->C O 2[h]+N A D P H[h]+R U 5 P[h]$ \\
\hline 89 & & $X 5 P[c]<=>R U 5 P[c]$ \\
\hline 90 & & $\mathrm{R} 5 \mathrm{P}[\mathrm{c}] \ll=>\mathrm{RU} 5 \mathrm{P}[\mathrm{c}]$ \\
\hline 91 & & $R 5 P[c]+X 5 P[c]<=>$ GAP $[c]+S 7 P[c]$ \\
\hline 92 & & $\mathrm{GAP}[\mathrm{c}]+\mathrm{S} 7 \mathrm{P}[\mathrm{c}] \Leftrightarrow=>\mathrm{F} 6 \mathrm{P}[\mathrm{c}]+\mathrm{E} 4 \mathrm{P}[\mathrm{c}]$ \\
\hline 93 & & $X 5 P[c]+E 4 P[c]<=>F 6 P[c]+G A P[c]$ \\
\hline 94 & 2.7.6.1 & $A T P[h]+R 5 P[h]->A M P[h]+H[h]+P R P P[h]$ \\
\hline 95 & 2.7.6.1 & $\mathrm{ATP}[\mathrm{c}]+\mathrm{R} 5 \mathrm{P}[\mathrm{c}]->\mathrm{AMP}[\mathrm{c}]+\mathrm{H}[\mathrm{c}]+\mathrm{PRPP}[\mathrm{c}]$ \\
\hline
\end{tabular}

\section{Sulphur assimilation}

$\begin{array}{rl}96 & 2.7 .7 .4 \\ 97 & 1.8 .4 .9 \\ 98 & 1.8 .1 .7 \\ 99 & 1.8 .7 .1 \\ 100 & 1.18 .1 .2\end{array}$

ATP $[h]+S O 4[h]->$ APS[h] + PPi[h]

APS $[h]+2$ gthrd[h] $\rightarrow$ AMP $[h]+\mathrm{SO} 3[h]+$ gthox $[h]$

$\mathrm{NADP}[\mathrm{h}]+2$ gthrd[h] $\Leftrightarrow \mathrm{NADPH}[\mathrm{h}]+$ gthox[h]

$6 \mathrm{H}[\mathrm{h}]+\mathrm{SO} 3[\mathrm{~h}]+6 \mathrm{fdxrd}[\mathrm{h}]->3 \mathrm{H} 2 \mathrm{O}[\mathrm{h}]+\mathrm{H} 2 \mathrm{~S}[\mathrm{~h}]+6 \mathrm{fdxox}[\mathrm{h}]$

$\mathrm{NADP}[\mathrm{h}]+2 \mathrm{fdxrd}[\mathrm{h}] \Leftrightarrow=>\mathrm{NADPH}[\mathrm{h}]+2 \mathrm{fdxox}[\mathrm{h}]$ 


\section{Amino acid metabolism}

101 1.1.1.95,

2.6.1.52,

3.1.3.3

102

103

104

105

106

109
4.3.1.17

2.3.1.30

2.5.1.47

3.3.1.1

2.5.1.54,

4.2.3.4,

4.2.1.10,

1.1.1.25,

2.7.1.71,

2.5.1.19,

4.2.3.5

5.4.99.5

4.2.1.51

2.6.1.9/

2.6.1.5/

2.6.1.1

1.3.1.12

2.6.1.5

4.1.3.27

2.4.2.18,

5.3.1.24,

4.1.1.48

4.2.1.20

2.7.2.11,

1.2.1.41

-

1.5.1.2

6.3.5.5

2.3.1.1,

2.7.2.8,

1.2.1.38

2.6.1.11

3.5.1.16

2.1.3.3

6.3.4.5,

4.3.2.1

2.6.1.1

2.6.1.1

6.3.5.4

2.7.2.4,

1.2.1.11

4.3.3.7,

1.17.1.8,

2.6.1.83,

5.1.1.7
$3 P G[h]+\mathrm{GLU}[\mathrm{h}]+\mathrm{H} 2 \mathrm{O}[\mathrm{h}]+\mathrm{NADP}[\mathrm{h}] \quad->\mathrm{AKG}[\mathrm{h}]+\mathrm{H}[\mathrm{h}]+\mathrm{NADPH}[\mathrm{h}]+\mathrm{Pi}[\mathrm{h}]+\mathrm{SER}[\mathrm{h}]$

SER[h] $->\mathrm{NH} 4[\mathrm{~h}]+\mathrm{PYR}[\mathrm{h}]$

AcCoA $[h]+$ SER $[h] \Leftrightarrow=$ ASER $[h]+\operatorname{CoA}[h]$

ASER $[h]+\mathrm{H} 2 \mathrm{~S}[\mathrm{~h}]+\mathrm{H}[\mathrm{h}]->\mathrm{Ac}[\mathrm{h}]+\mathrm{CYS}[\mathrm{h}]$

AdHCYS $[c]+\mathrm{H} 2 \mathrm{O}[\mathrm{c}] \Leftrightarrow \mathrm{Ad}[\mathrm{c}]+\mathrm{HCYS}[\mathrm{c}]$

$A T P[h]+E 4 P[h]+N A D P H[h]+2 P E P[h]->A D P[h]+C H O[h]+N A D P[h]+4 P i[h]$

$\mathrm{CHO}[\mathrm{h}] \Leftrightarrow=>\mathrm{PRE}[\mathrm{h}]$

$\mathrm{PRE}[\mathrm{c}]->\mathrm{CO} 2[\mathrm{c}]+\mathrm{H} 2 \mathrm{O}[\mathrm{c}]+\mathrm{PHPYR}[\mathrm{c}]$

GLU[c] + PHPYR $[c] \Leftrightarrow=A K G[c]+P H E[c]$

$\mathrm{NAD}[\mathrm{h}]+\mathrm{PRE}[\mathrm{h}] \Leftrightarrow=>4 \mathrm{HPHPYR}[\mathrm{h}]+\mathrm{CO} 2[\mathrm{~h}]+\mathrm{H}[\mathrm{h}]+\mathrm{NADH}[\mathrm{h}]$

4 HPHPYR $[\mathrm{h}]+\mathrm{GLU}[\mathrm{h}] \Leftrightarrow=\mathrm{AKG}[\mathrm{h}]+$ TYR $[\mathrm{h}]$

$\mathrm{CHO}[\mathrm{h}]+\mathrm{GLN}[\mathrm{h}] \Leftrightarrow=\mathrm{ANTH}[\mathrm{h}]+\mathrm{GLU}[\mathrm{h}]+\mathrm{PYR}[\mathrm{h}]$

ANTH[h] + PRPP[h] $->\mathrm{CO} 2[h]+\mathrm{H} 2 \mathrm{O}[\mathrm{h}]+\mathrm{I3GLYCP}[\mathrm{h}]+\mathrm{PPi}[\mathrm{h}]$

I3GLYCP[h] + SER[h] $\Leftrightarrow=$ GAP[h] + H2O[h] + TRYP[h]

$A T P[c]+\mathrm{GLU}[\mathrm{c}]+\mathrm{H}[\mathrm{c}]+\mathrm{NADPH}[\mathrm{c}] \Leftrightarrow=\mathrm{ADP}[\mathrm{c}]+\mathrm{GLUSAL}[\mathrm{c}]+\mathrm{NADP}[\mathrm{c}]+\mathrm{Pi}[\mathrm{c}]$

GLUSAL[c] $\Leftrightarrow=>P 5 C[c]$

$\mathrm{H}[\mathrm{c}]+\mathrm{NADPH}[\mathrm{c}]+\mathrm{P} 5 \mathrm{C}[\mathrm{c}]->\mathrm{NADP}[\mathrm{c}]+\mathrm{PRO}[\mathrm{c}]$

$2 \mathrm{ATP}[\mathrm{c}]+\mathrm{CO} 2[\mathrm{c}]+\mathrm{GLN}[\mathrm{c}]+2 \mathrm{H} 2 \mathrm{O}[\mathrm{c}]->2 \mathrm{ADP}[\mathrm{c}]+\mathrm{CaP}[\mathrm{c}]+\mathrm{GLU}[\mathrm{c}]+3 \mathrm{H}[\mathrm{c}]+\mathrm{Pi}[\mathrm{c}]$

$A T P[h]+A c C o A[h]+G L U[h]+H[h]+N A D P H[h] \Leftrightarrow=>A D P[h]+A c G L U S A L[h]+C o A[h]+$

NADP[h] + Pi[h]

AcGLUSAL[h] + GLU[h] $\Leftrightarrow$ AKG[h] + AcORN[h]

AcORN[h] $+\mathrm{H} 2 \mathrm{O}[\mathrm{h}] \Leftrightarrow \mathrm{Ac}[\mathrm{h}]+\mathrm{ORN}[\mathrm{h}]$

$\mathrm{CaP}[\mathrm{h}]+\mathrm{ORN}[\mathrm{h}] \Leftrightarrow \mathrm{CTU}[\mathrm{h}]+\mathrm{Pi}[\mathrm{h}]$

$A S P[h]+A T P[h]+C T U[h] \Leftrightarrow=A M P[h]+A R G[h]+F U M[h]+P P i[h]$

$\mathrm{GLU}[\mathrm{h}]+\mathrm{OXA}[\mathrm{h}] \Leftrightarrow=\mathrm{AKG}[\mathrm{h}]+\mathrm{ASP}[\mathrm{h}]$

$\mathrm{GLU}[\mathrm{m}]+\mathrm{OXA}[\mathrm{m}] \Leftrightarrow=\mathrm{AKG}[\mathrm{m}]+\mathrm{ASP}[\mathrm{m}]$

$\mathrm{ASP}[\mathrm{c}]+\mathrm{ATP}[\mathrm{c}]+\mathrm{GLN}[\mathrm{c}]+\mathrm{H} 2 \mathrm{O}[\mathrm{c}]-\mathrm{AMP}[\mathrm{c}]+\mathrm{ASN}[\mathrm{c}]+\mathrm{GLU}[\mathrm{c}]+\mathrm{H}[\mathrm{c}]+\mathrm{PPi}[\mathrm{c}]$

$A S P[h]+A T P[h]+H[h]+N A D P H[h] \Leftrightarrow=A D P[h]+A S A[h]+N A D P[h]+P i[h]$

$A S A[c]+\mathrm{GLU}[\mathrm{c}]+2 \mathrm{H}[\mathrm{c}]+\mathrm{NADPH}[\mathrm{c}]+\mathrm{PYR}[\mathrm{c}] \Leftrightarrow=\mathrm{AKG}[\mathrm{c}]+\mathrm{DAP}[\mathrm{c}]+\mathrm{H} 2 \mathrm{O}[\mathrm{c}]+\mathrm{NADP}[\mathrm{c}]$ 


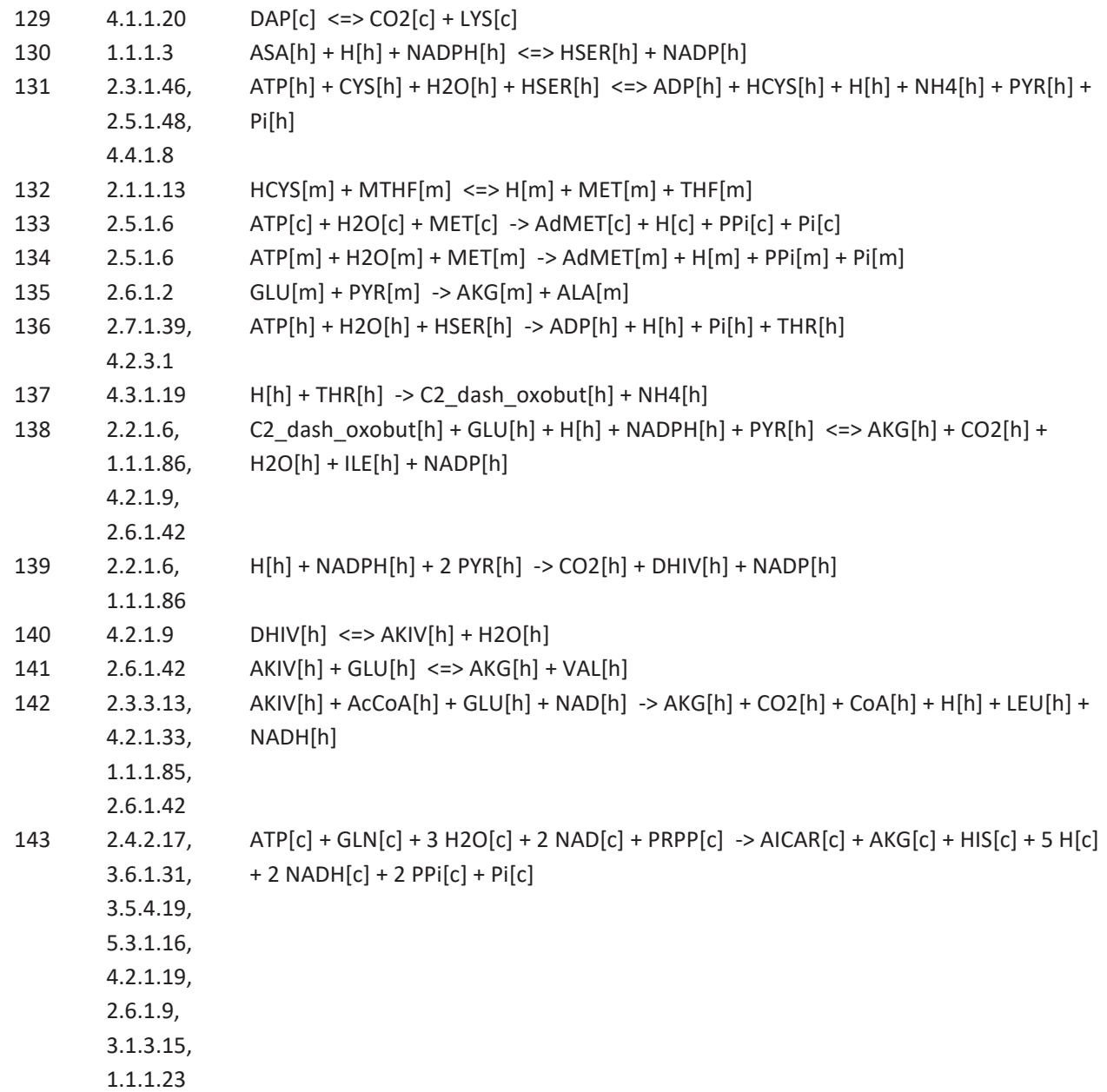

\section{Fermentation}

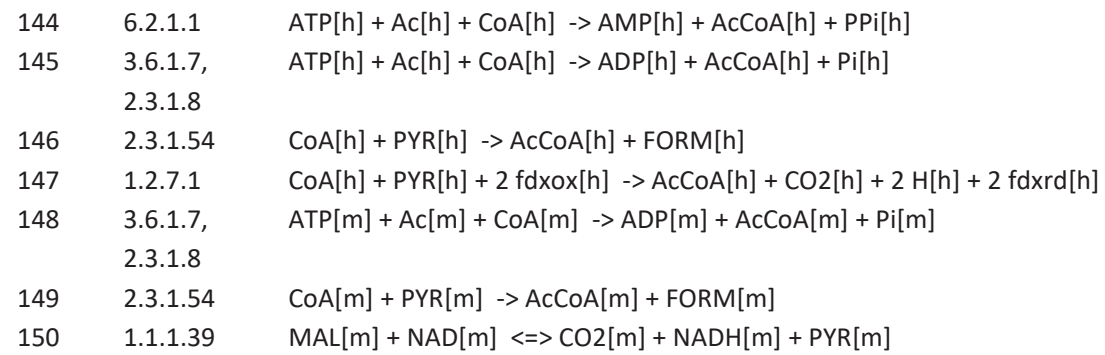

\section{Fatty acid biosynthesis}

$\begin{array}{lll}151 & 6.4 .1 .2 & \text { ATP }[h]+\mathrm{AcCoA}[\mathrm{h}]+\mathrm{CO} 2[\mathrm{~h}]+\mathrm{H} 2 \mathrm{O}[\mathrm{h}]->\mathrm{ADP}[\mathrm{h}]+\mathrm{H}[\mathrm{h}]+\mathrm{MalCoA}[\mathrm{h}]+\mathrm{Pi}[\mathrm{h}] \\ 152 & 2.3 .1 .38 & \mathrm{ACP}[\mathrm{h}]+\mathrm{AcCoA}[\mathrm{h}]+\mathrm{H}[\mathrm{h}]<=>\mathrm{AcACP}[\mathrm{h}]+\mathrm{CoA}[\mathrm{h}] \\ 153 & 2.3 .1 .39 & \mathrm{ACP}[\mathrm{h}]+\mathrm{MalCoA}[\mathrm{h}]<=>\operatorname{CoA}[\mathrm{h}]+\text { MalACP}[\mathrm{h}]\end{array}$


154 2.3.1.180, $\quad$ ACACP $[h]+12 \mathrm{H}[\mathrm{h}]+6 \mathrm{MalACP}[\mathrm{h}]+12 \mathrm{NADPH}[\mathrm{h}] \quad->6 \mathrm{ACP}[\mathrm{h}]+\mathrm{C} 140 \mathrm{ACP}[\mathrm{h}]+6$

1.1.1.100, $\quad \mathrm{CO} 2[\mathrm{~h}]+6 \mathrm{H} 2 \mathrm{O}[\mathrm{h}]+12 \mathrm{NADP}[\mathrm{h}]$

2.3.1.-,

1.3.1.10

AcACP $[\mathrm{h}]+14 \mathrm{H}[\mathrm{h}]+7 \mathrm{MalACP}[\mathrm{h}]+14 \mathrm{NADPH}[\mathrm{h}]->7 \mathrm{ACP}[\mathrm{h}]+\mathrm{C} 160 \mathrm{ACP}[\mathrm{h}]+7$

1.1.1.100, $\mathrm{CO} 2[\mathrm{~h}]+7 \mathrm{H} 2 \mathrm{O}[\mathrm{h}]+14 \mathrm{NADP}[\mathrm{h}]$

2.3.1.-

1.3.1.10

$156 \quad 2.3 .1 .180$,

$\mathrm{ACACP}[\mathrm{h}]+16 \mathrm{H}[\mathrm{h}]+8 \mathrm{MalACP}[\mathrm{h}]+16 \mathrm{NADPH}[\mathrm{h}]->8 \mathrm{ACP}[\mathrm{h}]+\mathrm{C} 180 \mathrm{ACP}[\mathrm{h}]+8$

1.1.1.100,

$\mathrm{CO} 2[\mathrm{~h}]+8 \mathrm{H} 2 \mathrm{O}[\mathrm{h}]+16 \mathrm{NADP}[\mathrm{h}]$

2.3.1.-

1.3.1.10

$157 \quad 1.14 .19 .2$

$\mathrm{C} 160 \mathrm{ACP}[\mathrm{h}]+\mathrm{H}[\mathrm{h}]+\mathrm{NADPH}[\mathrm{h}]+\mathrm{O} 2[\mathrm{~h}] \Leftrightarrow \mathrm{C} 161 \mathrm{ACP}[\mathrm{h}]+2 \mathrm{H} 2 \mathrm{O}[\mathrm{h}]+\mathrm{NADP}[\mathrm{h}]$

1.14.19.2

$\mathrm{C} 180 \mathrm{ACP}[\mathrm{h}]+\mathrm{H}[\mathrm{h}]+\mathrm{NADPH}[\mathrm{h}]+\mathrm{O} 2[\mathrm{~h}] \Leftrightarrow=\mathrm{C} 181 \mathrm{ACP}[\mathrm{h}]+2 \mathrm{H} 2 \mathrm{O}[\mathrm{h}]+\mathrm{NADP}[\mathrm{h}]$

$159 \quad 1.14 .19 .2$

$\mathrm{C} 161 \mathrm{ACP}[\mathrm{h}]+\mathrm{H}[\mathrm{h}]+\mathrm{NADPH}[\mathrm{h}]+\mathrm{O} 2[\mathrm{~h}] \Leftrightarrow=\mathrm{C} 162 \mathrm{ACP}[\mathrm{h}]+2 \mathrm{H} 2 \mathrm{O}[\mathrm{h}]+\mathrm{NADP}[\mathrm{h}]$

$160 \quad 1.14 .19 .1$

$\mathrm{C} 161 \mathrm{CoA}[\mathrm{c}]+\mathrm{H}[\mathrm{c}]+\mathrm{NADH}[\mathrm{c}]+\mathrm{O} 2[\mathrm{c}] \Leftrightarrow \mathrm{C} 162 \mathrm{CoA}[\mathrm{c}]+2 \mathrm{H} 2 \mathrm{O}[\mathrm{c}]+\mathrm{NAD}[\mathrm{c}]$

$161 \quad 1.14 .19 .2$

$162 \quad-$

$163 \quad 1.14 .19 .2$

$164 \quad 1.14 .19 .2$

$165 \quad 3.1 .2 .14$

$166 \quad 3.1 .2 .14$

$167 \quad 3.1 .2 .14$

$168 \quad 3.1 .2 .14$

$169 \quad 6.2 .1 .3$

170 -

171 -

172 -

$173 \quad 1.1 .1 .94$

$\mathrm{C} 181 \mathrm{ACP}[\mathrm{h}]+\mathrm{H}[\mathrm{h}]+\mathrm{NADPH}[\mathrm{h}]+\mathrm{O} 2[\mathrm{~h}] \Leftrightarrow \mathrm{C} 182 \mathrm{ACP}[\mathrm{h}]+2 \mathrm{H} 2 \mathrm{O}[\mathrm{h}]+\mathrm{NADP}[\mathrm{h}]$

$\mathrm{C} 181 \mathrm{CoA}[\mathrm{c}]+\mathrm{H}[\mathrm{c}]+\mathrm{NADH}[\mathrm{c}]+\mathrm{O} 2[\mathrm{c}] \Leftrightarrow=\mathrm{C} 182 \mathrm{CoA}[\mathrm{c}]+2 \mathrm{H} 2 \mathrm{O}[\mathrm{c}]+\mathrm{NAD}[\mathrm{c}]$

$\mathrm{C} 162 \mathrm{ACP}[\mathrm{h}]+\mathrm{H}[\mathrm{h}]+\mathrm{NADPH}[\mathrm{h}]+\mathrm{O} 2[\mathrm{~h}] \Leftrightarrow \mathrm{C} 163 \mathrm{ACP}[\mathrm{h}]+2 \mathrm{H} 2 \mathrm{O}[\mathrm{h}]+\mathrm{NADP}[\mathrm{h}]$

$\mathrm{C} 182 \mathrm{ACP}[\mathrm{h}]+\mathrm{H}[\mathrm{h}]+\mathrm{NADPH}[\mathrm{h}]+\mathrm{O} 2[\mathrm{~h}] \Leftrightarrow \mathrm{C} 183 \mathrm{ACP}[\mathrm{h}]+2 \mathrm{H} 2 \mathrm{O}[\mathrm{h}]+\mathrm{NADP}[\mathrm{h}]$

$\mathrm{C} 160 \mathrm{ACP}[\mathrm{h}]+\mathrm{H} 2 \mathrm{O}[\mathrm{h}] \Leftrightarrow=\mathrm{ACP}[\mathrm{h}]+\mathrm{C} 160[\mathrm{~h}]+\mathrm{H}[\mathrm{h}]$

$\mathrm{C} 161 \mathrm{ACP}[\mathrm{h}]+\mathrm{H} 2 \mathrm{O}[\mathrm{h}]<=>\mathrm{ACP}[\mathrm{h}]+\mathrm{C} 161[\mathrm{~h}]+\mathrm{H}[\mathrm{h}]$

$\mathrm{C} 180 \mathrm{ACP}[\mathrm{h}]+\mathrm{H} 2 \mathrm{O}[\mathrm{h}]<=>\mathrm{ACP}[\mathrm{h}]+\mathrm{C} 180[\mathrm{~h}]+\mathrm{H}[\mathrm{h}]$

$\mathrm{C} 181 \mathrm{ACP}[\mathrm{h}]+\mathrm{H} 2 \mathrm{O}[\mathrm{h}] \Leftrightarrow=\mathrm{ACP}[\mathrm{h}]+\mathrm{C} 181[\mathrm{~h}]+\mathrm{H}[\mathrm{h}]$

$\mathrm{ATP}[\mathrm{c}]+\mathrm{C} 160[\mathrm{c}]+\mathrm{CoA}[\mathrm{c}]->\mathrm{AMP}[\mathrm{c}]+\mathrm{C} 160 \mathrm{CoA}[\mathrm{c}]+\mathrm{PPi}[\mathrm{c}]$

$\mathrm{ATP}[\mathrm{c}]+\mathrm{C} 161[\mathrm{c}]+\mathrm{CoA}[\mathrm{c}] \rightarrow \mathrm{AMP}[\mathrm{c}]+\mathrm{C} 161 \mathrm{CoA}[\mathrm{c}]+\mathrm{PPi}[\mathrm{c}]$

$\mathrm{ATP}[\mathrm{c}]+\mathrm{C} 180[\mathrm{c}]+\mathrm{CoA}[\mathrm{c}]-\rightarrow \mathrm{AMP}[\mathrm{c}]+\mathrm{C} 180 \mathrm{CoA}[\mathrm{c}]+\mathrm{PPi}[\mathrm{c}]$

$\mathrm{ATP}[\mathrm{c}]+\mathrm{C} 181[\mathrm{c}]+\mathrm{CoA}[\mathrm{c}]->\mathrm{AMP}[\mathrm{c}]+\mathrm{C} 181 \mathrm{CoA}[\mathrm{c}]+\mathrm{PPi}[\mathrm{c}]$

GLYC3P[h] + NADP[h] -> DHAP[h] + H[h] + NADPH[h]

\section{Purine and Pyrimidine metabolism}

$174 \quad 2.4 .2 .14$,

6.3.4.13,

2.1.2.2,

6.3.5.3,

6.3.3.1,

4.1.1.21,

6.3.2.6,

4.3.2.2

175

2.1.2.3,

3.5.4.10

176 6.3.4.4,

4.3.2.2

177

1.1.1.205,

(6.3.4.1)

6.3.5.2)

178

2.7.4.8

2.7.4.6
$\mathrm{ASP}[\mathrm{c}]+4 \mathrm{ATP}[\mathrm{c}]+\mathrm{CO} 2[\mathrm{c}]+2 \mathrm{GLN}[\mathrm{c}]+\mathrm{GLY}[\mathrm{c}]+2 \mathrm{H} 2 \mathrm{O}[\mathrm{c}]+\mathrm{N} 10 \mathrm{FTHF}[\mathrm{c}]+\mathrm{PRPP}[\mathrm{c}]$-> $4 \mathrm{ADP}[\mathrm{c}]+\mathrm{AICAR}[\mathrm{c}]+\mathrm{FUM}[\mathrm{c}]+2 \mathrm{GLU}[\mathrm{c}]+7 \mathrm{H}[\mathrm{c}]+\mathrm{PPi}[\mathrm{c}]+4 \mathrm{Pi}[\mathrm{c}]+\mathrm{THF}[\mathrm{c}]$

$\operatorname{AICAR}[\mathrm{c}]+\mathrm{N} 10 \mathrm{FTHF}[\mathrm{c}] \Leftrightarrow=>\mathrm{H} 2 \mathrm{O}[\mathrm{c}]+\mathrm{IMP}[\mathrm{c}]+\mathrm{THF}[\mathrm{c}]$

$A S P[c]+G T P[c]+I M P[c] \Leftrightarrow=>A M P[c]+F U M[c]+G D P[c]+P i[c]$

$\mathrm{ATP}[\mathrm{c}]+\mathrm{GLN}[\mathrm{c}]+2 \mathrm{H} 2 \mathrm{O}[\mathrm{c}]+\mathrm{IMP}[\mathrm{c}]+\mathrm{NAD}[\mathrm{c}]-\mathrm{AMP}[\mathrm{c}]+\mathrm{GLU}[\mathrm{c}]+\mathrm{GMP}[\mathrm{c}]+3 \mathrm{H}[\mathrm{c}]+$ $\mathrm{NADH}[\mathrm{c}]+\mathrm{PPi}[\mathrm{c}]$

$A T P[c]+G M P[c] \rightarrow A D P[c]+G D P[c]$

$A T P[c]+G D P[c] \Leftrightarrow=A D P[c]+G T P[c]$ 


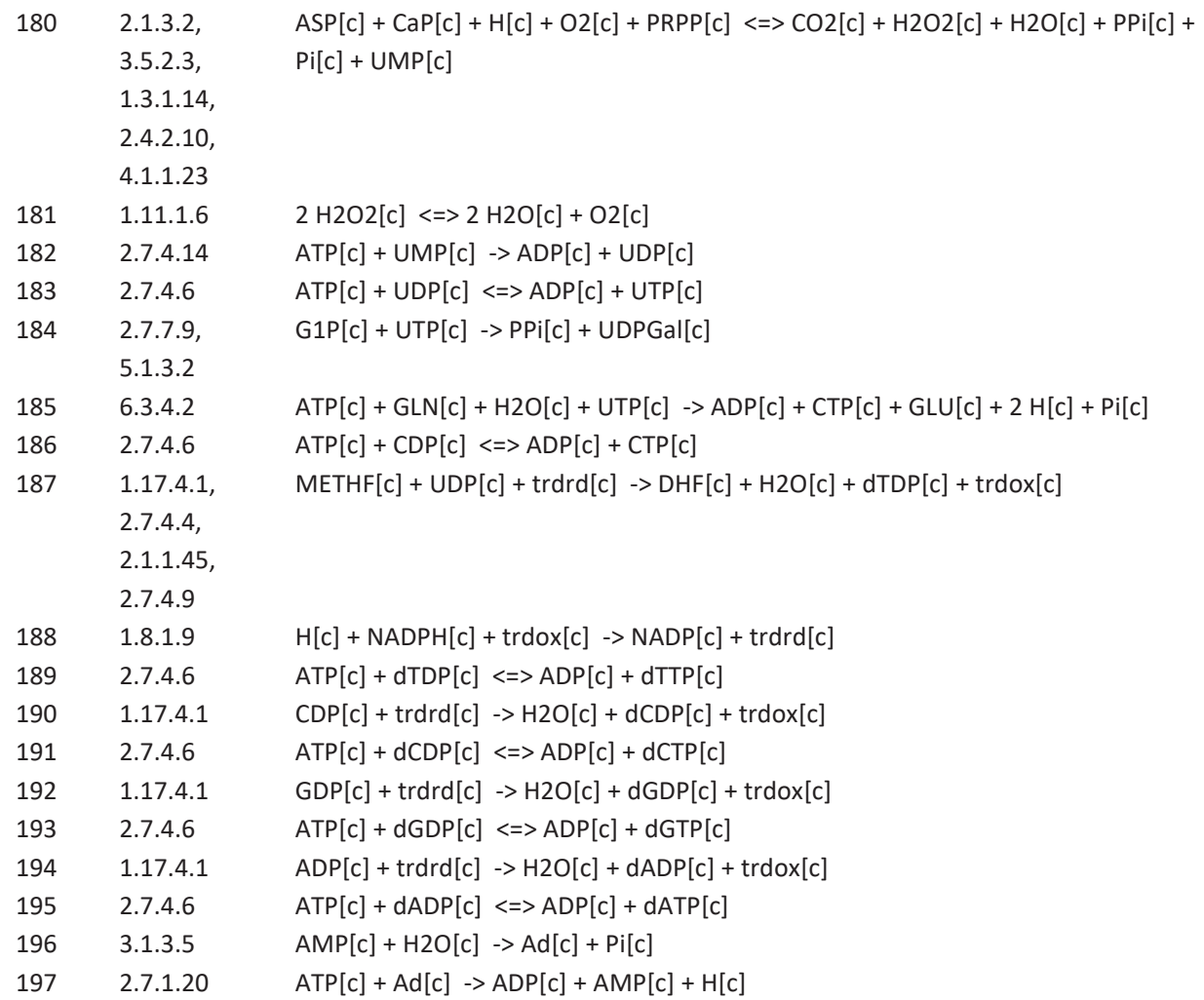

\section{Folate metabolism}

\begin{tabular}{|c|c|c|}
\hline 198 & 2.1.2.10 & $5 \mathrm{FTHF}[\mathrm{c}]+\mathrm{H}[\mathrm{c}] \Leftrightarrow=>\mathrm{H} 2 \mathrm{O}[\mathrm{c}]+\mathrm{MYLTHF}[\mathrm{c}]$ \\
\hline 199 & 3.5.4.9 & $\mathrm{H} 2 \mathrm{O}[\mathrm{c}]+\mathrm{MYLTHF}[\mathrm{c}] \Leftrightarrow=>\mathrm{H}[\mathrm{c}]+\mathrm{N} 10 \mathrm{FTHF}[\mathrm{c}]$ \\
\hline 200 & 3.5.4.9 & $\mathrm{H} 2 \mathrm{O}[\mathrm{m}]+\mathrm{MYLTHF}[\mathrm{m}]<=>\mathrm{H}[\mathrm{m}]+\mathrm{N} 10 \mathrm{FTHF}[\mathrm{m}]$ \\
\hline 201 & 6.3.4.3 & $A T P[c]+F O R M[c]+\operatorname{THF}[c]->A D P[c]+N 10 F T H F[c]+P i[c]$ \\
\hline 202 & 6.3.4.3 & ATP $[m]+F O R M[m]+\operatorname{THF}[m] \Leftrightarrow=>A D P[m]+N 10 F T H F[m]+P i[m]$ \\
\hline 203 & 1.5.1.5 & $H[c]+\operatorname{MYLTHF}[c]+N A D P H[c] \Leftrightarrow=>\operatorname{METHF}[c]+N A D P[c]$ \\
\hline 204 & 1.5.1.5 & $H[m]+M Y L T H F[m]+N A D H[m] \Leftrightarrow=\operatorname{METHF[m]+NAD[m]}$ \\
\hline 205 & 1.5.1.20 & $H[c]+\operatorname{METHF}[c]+N A D P H[c] \Leftrightarrow=>M T H F[c]+N A D P[c]$ \\
\hline 206 & $\begin{array}{l}\text { 6.3.3.2, } \\
3.5 .4 .9 / \\
2.1 .2 .2\end{array}$ & $5 \mathrm{FTHF}[\mathrm{c}]+\mathrm{ATP}[\mathrm{c}]+\mathrm{H} 2 \mathrm{O}[\mathrm{c}]->\mathrm{ADP}[\mathrm{c}]+\mathrm{H}[\mathrm{c}]+\mathrm{N} 10 \mathrm{FTHF}[\mathrm{c}]+\mathrm{Pi}[\mathrm{c}]$ \\
\hline 207 & 3.5.1.10 & $\mathrm{FORM}[\mathrm{c}]+\mathrm{H}[\mathrm{c}]+\mathrm{THF}[\mathrm{c}] \Leftrightarrow=>\mathrm{H} 2 \mathrm{O}[\mathrm{c}]+\mathrm{N} 10 \mathrm{FTHF}[\mathrm{c}]$ \\
\hline 208 & 1.5.1.3 & $\mathrm{DHF}[\mathrm{c}]+\mathrm{H}[\mathrm{c}]+\mathrm{NADPH}[\mathrm{c}] \Leftrightarrow=\mathrm{NADP}[\mathrm{c}]+\mathrm{THF}[\mathrm{c}]$ \\
\hline
\end{tabular}

\section{Pigment synthesis}

$8 \mathrm{ATP}[\mathrm{h}]+8 \mathrm{GLU}[\mathrm{h}]+12 \mathrm{H}[\mathrm{h}]+8 \mathrm{NADPH}[\mathrm{h}]+2.5 \mathrm{O} 2[\mathrm{~h}]->8 \mathrm{AMP}[\mathrm{h}]+6 \mathrm{CO} 2[\mathrm{~h}]+13$ $\mathrm{H} 2 \mathrm{O}[\mathrm{h}]+8 \mathrm{NADP}[\mathrm{h}]+4 \mathrm{NH} 4[\mathrm{~h}]+8 \mathrm{PPi}[\mathrm{h}]+$ PPorphyr[h] 
2 ATP[h] + AdMET[h] + Mg2[h] + 4 NADPH[h] + 2.5 O2[h] + PPorphyr[h] + Phytyl_dash_PP[h] -> 2 ADP[h] + AdHCYS[h] + Chlorophyl[h] + $2 \mathrm{H} 2 \mathrm{O}[\mathrm{h}]+3 \mathrm{H}[\mathrm{h}]+4$ $\mathrm{NADP}[\mathrm{h}]+\mathrm{PPi}[\mathrm{h}]+2 \mathrm{Pi}[\mathrm{h}]$ $6 \mathrm{NADP}[\mathrm{h}]+2 \mathrm{O} 2[\mathrm{~h}]+2$ Phytyl_dash_PP[h] -> Carotene[h] + $4 \mathrm{H} 2 \mathrm{O}[\mathrm{h}]+12 \mathrm{H}[\mathrm{h}]+6$ $\mathrm{NADPH}[\mathrm{h}]+2 \mathrm{PPi}[\mathrm{h}]$

\section{Macromolecules and functional biomass formation}

213
$\mathrm{G} 1 \mathrm{P}[\mathrm{c}]->\mathrm{CARB}[\mathrm{c}]+\mathrm{Pi}[\mathrm{c}]$

$0.6 \mathrm{ATP}[\mathrm{c}]+0.3 \mathrm{CTP}[\mathrm{c}]+0.3 \mathrm{GTP}[\mathrm{c}]+1.4 \mathrm{H} 2 \mathrm{O}[\mathrm{c}]+0.2 \mathrm{UTP}[\mathrm{c}]->0.4 \mathrm{ADP}[\mathrm{c}]+0.4 \mathrm{H}[\mathrm{c}]$ $+\mathrm{PPi}[\mathrm{c}]+0.4 \mathrm{Pi}[\mathrm{c}]+\mathrm{RNA}[\mathrm{c}]$

$1.372 \mathrm{ATP}[\mathrm{c}]+2.372 \mathrm{H} 2 \mathrm{O}[\mathrm{c}]+0.2 \mathrm{dATP}[\mathrm{c}]+0.3 \mathrm{dCTP}[\mathrm{c}]+0.3 \mathrm{dGTP}[\mathrm{c}]+0.2 \mathrm{dTTP}[\mathrm{c}]$-> $1.372 \mathrm{ADP}[\mathrm{c}]+\mathrm{DNA}[\mathrm{c}]+2.372 \mathrm{H}[\mathrm{c}]+\mathrm{PPi}[\mathrm{c}]+1.372 \mathrm{Pi}[\mathrm{c}]$

$0.142 \mathrm{ALA}[\mathrm{c}]+0.05 \mathrm{ARG}[\mathrm{c}]+0.042 \mathrm{ASN}[\mathrm{c}]+0.042 \mathrm{ASP}[\mathrm{c}]+4.306 \mathrm{ATP}[\mathrm{c}]+0.015$ $\mathrm{CYS}[\mathrm{c}]+0.054 \mathrm{GLN}[\mathrm{c}]+0.054 \mathrm{GLU}[\mathrm{c}]+0.099 \mathrm{GLY}[\mathrm{c}]+3.306 \mathrm{H} 2 \mathrm{O}[\mathrm{c}]+0.016 \mathrm{HIS}[\mathrm{c}]+$ $0.037 \mathrm{ILE}[\mathrm{c}]+0.085 \mathrm{LEU}[\mathrm{c}]+0.054 \mathrm{LYS}[\mathrm{c}]+0.021 \mathrm{MET}[\mathrm{c}]+0.037 \mathrm{PHE}[\mathrm{c}]+0.052$ $\mathrm{PRO}[\mathrm{c}]+0.052 \mathrm{SER}[\mathrm{c}]+0.049 \mathrm{THR}[\mathrm{c}]+0.01 \mathrm{TRYP}[\mathrm{c}]+0.024 \mathrm{TYR}[\mathrm{c}]+0.063 \mathrm{VAL}[\mathrm{c}]$-> 4.306 ADP $[\mathrm{c}]+4.306 \mathrm{H}[\mathrm{c}]+\mathrm{PROTEIN}[\mathrm{c}]+4.306 \mathrm{Pi}[\mathrm{c}]$ $0.05 \mathrm{C} 140 \mathrm{ACP}[\mathrm{h}]+0.53 \mathrm{C} 160 \mathrm{ACP}[\mathrm{h}]+0.03 \mathrm{C161ACP}[\mathrm{h}]+0.08 \mathrm{C162ACP}[\mathrm{h}]+0.1$ C163ACP $[h]+0.01 C 180 A C P[h]+0.23 C 181 A C P[h]+0.63 C 182 A C P[h]+0.34$ C183ACP $[h]+$ GLYC3P[h] + H2O[h] + UDPGal[h] -> $2 A C P[h]+2 H[h]+M G D G[h]+$ $\mathrm{Pi}[\mathrm{h}]+\mathrm{UDP}[\mathrm{h}]$

$0.56 \mathrm{C160ACP}[\mathrm{h}]+0.06 \mathrm{C161ACP}[\mathrm{h}]+0.11 \mathrm{C162ACP}[\mathrm{h}]+0.05 \mathrm{C180ACP}[\mathrm{h}]+1.38$ C181ACP $[h]+0.84$ C182ACP $[h]+\mathrm{GLYC3P}[\mathrm{h}]+\mathrm{H} 2 \mathrm{O}[\mathrm{h}]->3 \mathrm{ACP}[\mathrm{h}]+3 \mathrm{H}[\mathrm{h}]+\mathrm{Pi}[\mathrm{h}]+$ TAG[h]

$0.56 \mathrm{C} 160 \mathrm{CoA}[\mathrm{c}]+0.06 \mathrm{C} 161 \mathrm{CoA}[\mathrm{c}]+0.11 \mathrm{C} 162 \mathrm{CoA}[\mathrm{c}]+0.05 \mathrm{C} 180 \mathrm{CoA}[\mathrm{c}]+1.38$ $\mathrm{C} 181 \mathrm{CoA}[\mathrm{c}]+0.84 \mathrm{C} 182 \mathrm{CoA}[\mathrm{c}]+\mathrm{GLYC} 3 \mathrm{P}[\mathrm{c}]+\mathrm{H} 2 \mathrm{O}[\mathrm{c}]->3 \mathrm{CoA}[\mathrm{c}]+3 \mathrm{H}[\mathrm{c}]+\mathrm{Pi}[\mathrm{c}]+$ TAG[c]

121.02 ATP $[c]+0.7887$ CARB $[c]+0.0141$ Carotene $[c]+0.0433$ Chlorophyl[c] + 0.0056 DNA $[\mathrm{c}]+121.02 \mathrm{H} 2 \mathrm{O}[\mathrm{c}]+0.0849 \mathrm{MGDG}[\mathrm{c}]+5.8576 \mathrm{PROTEIN}[\mathrm{c}]+0.1769 \mathrm{RNA}[\mathrm{c}]$-> 121.02 ADP[c] + Biomass_ $x[c]+121.02 \mathrm{H}[\mathrm{c}]+121.02 \mathrm{Pi}[\mathrm{c}]$

\section{Transport chloroplast}

photon[c] $\rightarrow>$ photon $[\mathrm{h}]$

fluores[h] $\rightarrow>$ fluores[c]

$\mathrm{O} 2[\mathrm{~h}] \Leftrightarrow=\mathrm{O} 2[\mathrm{c}]$

$\mathrm{H} 2 \mathrm{O}[\mathrm{h}]<=>\mathrm{H} 2 \mathrm{O}[\mathrm{c}]$

$\mathrm{H}[\mathrm{h}]<=>\mathrm{H}[\mathrm{c}]$

$\mathrm{CO} 2[\mathrm{~h}] \Leftrightarrow \mathrm{CO} 2[\mathrm{c}]$

GAP $[\mathrm{h}] \Leftrightarrow$ GAP $[\mathrm{c}]$

$3 P G[h]<=>3 P G[c]$

$A D P[h]+A T P[c]<=>A D P[c]+A T P[h]$

$\mathrm{G} 1 \mathrm{P}[\mathrm{h}]+\mathrm{Pi}[\mathrm{c}] \Leftrightarrow=\mathrm{G} 1 \mathrm{P}[\mathrm{c}]+\mathrm{Pi}[\mathrm{h}]$

STARCH[h] $\Leftrightarrow$ STARCH[c]

$\mathrm{NO} 2[\mathrm{~h}] \Leftrightarrow=>\mathrm{NO} 2[\mathrm{c}]$

$\mathrm{NH} 4[\mathrm{~h}] \Leftrightarrow=>\mathrm{NH} 4[\mathrm{c}]$

$A K G[c]+G L U[h] \Leftrightarrow=A K G[h]+G L U[c]$

$\mathrm{GLU}[\mathrm{h}] \Leftrightarrow \mathrm{GLU}[\mathrm{c}]$

GLN[h] $\Leftrightarrow$ GLN[c]

$\mathrm{SO} 4[\mathrm{~h}] \Leftrightarrow=>\mathrm{SO} 4[\mathrm{c}]$ 


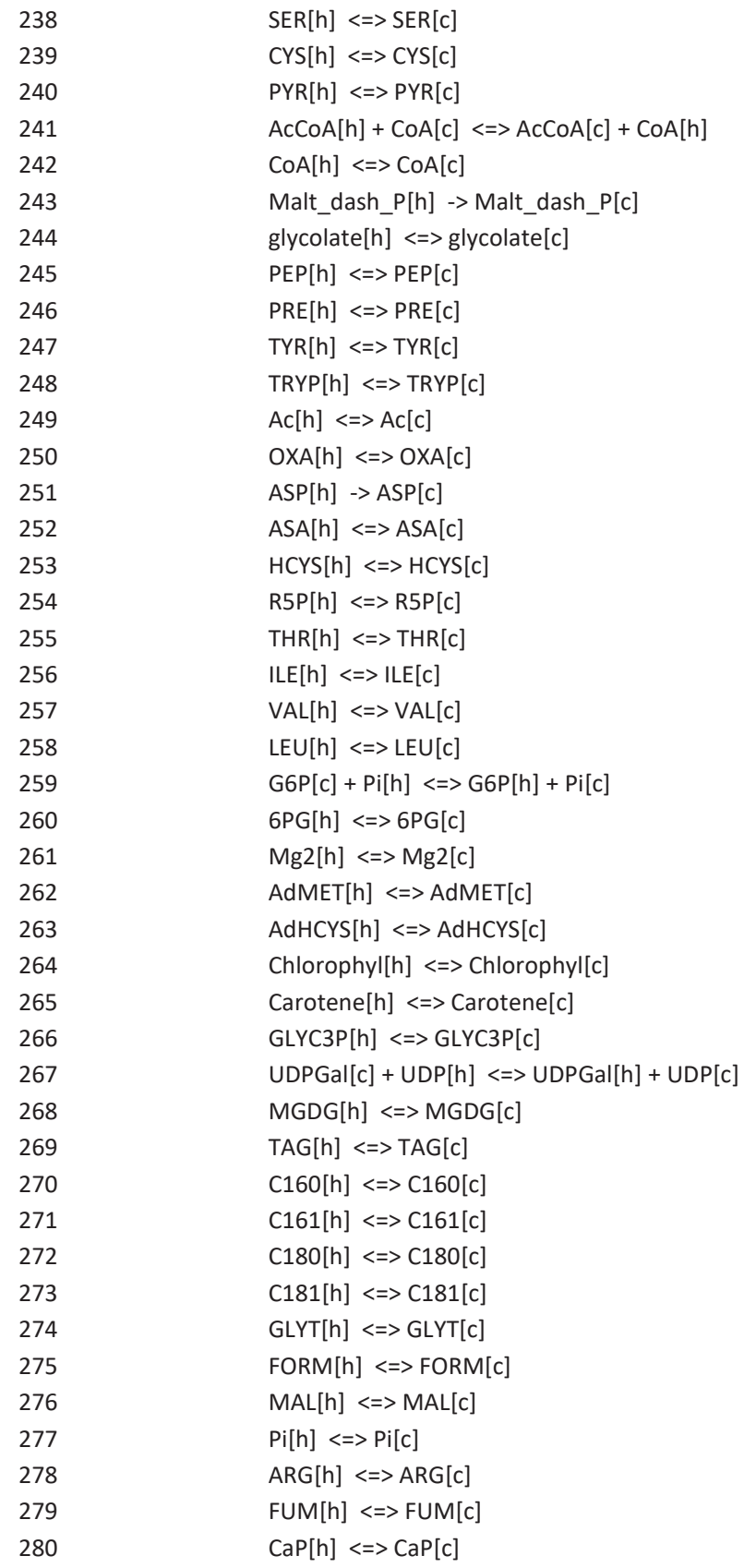

\section{Transport mitochondria}

281

282

283

284
GLYX[c] $\Leftrightarrow=$ GLYX[m]

$\operatorname{PYR}[\mathrm{c}] \Leftrightarrow \operatorname{PYR}[\mathrm{m}]$

$\mathrm{CO} 2[\mathrm{c}] \Leftrightarrow=>\mathrm{CO} 2[\mathrm{~m}]$

CIT $[\mathrm{c}] \Leftrightarrow=>$ CIT[m] 
$\mathrm{H}[\mathrm{c}] \Leftrightarrow \mathrm{H}[\mathrm{m}]$

$\mathrm{H} 2 \mathrm{O}[\mathrm{c}] \Leftrightarrow=\mathrm{H} 2 \mathrm{O}[\mathrm{m}]$

$A D P[c]+A T P[m] \Leftrightarrow A D P[m]+A T P[c]$

$\operatorname{MAL}[\mathrm{m}]+\mathrm{OXA}[\mathrm{c}] \Leftrightarrow=>\operatorname{MAL}[\mathrm{c}]+\mathrm{OXA}[\mathrm{m}]$

$\operatorname{MAL}[\mathrm{c}]->\operatorname{MAL}[\mathrm{m}]$

$\mathrm{H}[\mathrm{c}]+\mathrm{Pi}[\mathrm{c}] \Leftrightarrow=\mathrm{H}[\mathrm{m}]+\mathrm{Pi}[\mathrm{m}]$

$\mathrm{O} 2[\mathrm{c}] \Leftrightarrow=\mathrm{O} 2[\mathrm{~m}]$

glycolate[c] $<=>$ glycolate[m]

ALA $[\mathrm{c}] \Leftrightarrow=>$ ALA $[\mathrm{m}]$

$\mathrm{GLY}[\mathrm{c}] \Leftrightarrow=\mathrm{GLY}[\mathrm{m}]$

METHF[c] $\rightarrow$ METHF[m]

THF[c] $\Leftrightarrow=>$ THF[m]

$\mathrm{SER}[\mathrm{c}] \Leftrightarrow \mathrm{SER}[\mathrm{m}]$

$\mathrm{NH} 4[\mathrm{c}] \Leftrightarrow \mathrm{NH} 4[\mathrm{~m}]$

GLYT[c] $\Leftrightarrow=$ GLYT[m]

FUM[m] $\Leftrightarrow$ FUM[c]

$\operatorname{HCYS}[\mathrm{c}] \Leftrightarrow=\operatorname{HCYS}[\mathrm{m}]$

MTHF[c] $\Leftrightarrow$ MTHF[m]

$\operatorname{MET}[\mathrm{c}] \Leftrightarrow \operatorname{MET}[\mathrm{m}]$

$\mathrm{GLU}[\mathrm{c}] \Leftrightarrow=>\mathrm{GLU}[\mathrm{m}]$

$A K G[m]+M A L[c] \Leftrightarrow=A K G[c]+M A L[m]$

$\operatorname{AdMET}[\mathrm{c}] \Leftrightarrow=>\operatorname{AdMET}[\mathrm{m}]$

$\mathrm{AcCoA}[\mathrm{c}]+\mathrm{CoA}[\mathrm{m}] \Leftrightarrow=\mathrm{AcCoA}[\mathrm{m}]+\operatorname{CoA}[\mathrm{c}]$

$\mathrm{Ac}[\mathrm{c}] \Leftrightarrow \mathrm{Ac}[\mathrm{m}]$

FORM $[\mathrm{c}] \Leftrightarrow=>$ FORM $[\mathrm{m}]$

ASP $[\mathrm{c}] \Leftrightarrow$ ASP $[\mathrm{m}]$

$\mathrm{SUC}[\mathrm{c}]<=>\mathrm{SUC}[\mathrm{m}]$

\section{Extracellular transport}

312

313

314

315

316

317

318

319

320

321

322

323

324

325

326

327

328

329

330

331

photon[e] $\rightarrow>$ photon[c]

fluores[c] $\rightarrow>$ fluores[e]

$\mathrm{O} 2[\mathrm{e}] \Leftrightarrow=\mathrm{O}$ [c]

$\mathrm{ATP}[\mathrm{c}]+\mathrm{H} 2 \mathrm{O}[\mathrm{c}]+\mathrm{Pi}[\mathrm{e}]->\mathrm{ADP}[\mathrm{c}]+\mathrm{H}[\mathrm{c}]+2 \mathrm{Pi}[\mathrm{c}]$

$\mathrm{H} 2 \mathrm{O}[\mathrm{e}]<=>\mathrm{H} 2 \mathrm{O}[\mathrm{c}]$

$\mathrm{H}[\mathrm{e}]<=>\mathrm{H}[\mathrm{c}]$

$\mathrm{CO} 2[\mathrm{e}] \Leftrightarrow=>\mathrm{CO}[\mathrm{c}]$

STARCH[e] $\Leftrightarrow$ STARCH[c]

CARB $[\mathrm{e}] \Leftrightarrow=>\mathrm{CARB}[\mathrm{c}]$

$\mathrm{ATP}[\mathrm{c}]+\mathrm{H} 2 \mathrm{O}[\mathrm{c}]+\mathrm{NO} 3[\mathrm{e}]->\mathrm{ADP}[\mathrm{c}]+\mathrm{H}[\mathrm{c}]+\mathrm{NO} 3[\mathrm{c}]+\mathrm{Pi}[\mathrm{c}]$

$\mathrm{ATP}[\mathrm{c}]+\mathrm{H} 2 \mathrm{O}[\mathrm{c}]+\mathrm{SO} 4[\mathrm{e}]->\mathrm{ADP}[\mathrm{c}]+\mathrm{H}[\mathrm{c}]+\mathrm{Pi}[\mathrm{c}]+\mathrm{SO} 4[\mathrm{c}]$

RNA[c] $\Leftrightarrow$ RNA[e]

DNA[c] $\Leftrightarrow$ DNA[e]

PROTEIN[c] $\Leftrightarrow=>$ PROTEIN[e]

Mg2[c] $\Leftrightarrow$ Mg2[e]

Chlorophyl[c] $\Leftrightarrow=>$ Chlorophyl[e]

Carotene[c] $\Leftrightarrow=>$ Carotene[e]

MGDG[c] $\Leftrightarrow$ MGDG[e]

TAG $[\mathrm{c}] \Leftrightarrow=>\mathrm{TAG}[\mathrm{e}]$

Biomass_x[c] $\Leftrightarrow=>$ Biomass_x[e] 


\section{Exchange reactions}

$\begin{array}{ll}332 & \text { photon[e] }<=> \\ 333 & \text { fluores[e] }-> \\ 334 & \text { O2[e] <=> } \\ 335 & \text { Pi[e] <=> } \\ 336 & \mathrm{H} 2 \mathrm{O}[\mathrm{e}]<=> \\ 337 & \mathrm{H}[\mathrm{e}]<=> \\ 338 & \text { CO2[e] <=> } \\ 339 & \text { NO3[e] <=> } \\ 340 & \text { STARCH[e] -> } \\ 341 & \text { CARB[e] -> } \\ 342 & \text { SO4[e] <=> } \\ 343 & \text { RNA[e] }-> \\ 344 & \text { DNA[e] -> } \\ 345 & \text { PROTEIN[e] -> } \\ 346 & \text { Mg2[e] <=> } \\ 347 & \text { Chlorophyl[e] -> } \\ 348 & \text { Carotene[e] -> } \\ 349 & \text { MGDG[e] -> } \\ 350 & \text { TAG[e] -> } \\ 351 & \text { Biomass_x[e] -> }\end{array}$

\section{Supplementary File S6.2. Metabolite abbreviations}

\begin{tabular}{|c|c|c|c|}
\hline 13DPG & 1,3-diphosphoglycerate & GDP & Guanosine diphosphate \\
\hline 2ahethmpp & $\begin{array}{l}\text { 2-(alpha-Hydroxyethyl)thiamine } \\
\text { diphosphate }\end{array}$ & GLN & Glutamine \\
\hline $2 P G$ & 2-Phosophoglycerate & GLPG & $\begin{array}{l}\text { D-Glucono-1,5-lactone 6- } \\
\text { phosphate }\end{array}$ \\
\hline 3PG & 3-phosphoglycerate & GLU & Glutamate \\
\hline 4HPHPYR & 3-(4-Hydroxyphenyl)pyruvate & GLUSAL & $\begin{array}{l}\text { L-Glutamate 5- } \\
\text { semialdehyde }\end{array}$ \\
\hline $5 F T H F$ & 5-Formyl-THF & GLY & Glycine \\
\hline $6 P G$ & 6-Phosphogluconate & GLYC & Glycerol \\
\hline Ac & Acetate & GLYC3P & Glycerol 3-phosphate \\
\hline AcACP & Acetyl-ACP & glycolate & Glycolate \\
\hline $\mathrm{AcCoA}$ & Acetyl-CoA & GLYT & Glycerate \\
\hline AcGLUSAL & N-Acetyl-L-glutamate 5-semialdehyde & GLYX & Glyoxylate \\
\hline AcORN & N-Acetylornithine & GMP & $\begin{array}{l}\text { Guanosine } \\
\text { monophosphate }\end{array}$ \\
\hline $\mathrm{ACP}$ & Acetyl Carrier Protein & gthox & glutathione disulfide \\
\hline Ad & Adenosine & gthrd & glutathione \\
\hline AdHCYS & S-Adenosyl-L-homocysteine & GTP & Guanosine triphosphate \\
\hline
\end{tabular}




\begin{tabular}{|c|c|c|c|}
\hline adhlam & $\begin{array}{l}\text { [Dihydrolipoyllysine-residue } \\
\text { acetyltransferase] S- } \\
\text { acetyldihydrolipoyllysine }\end{array}$ & $\mathrm{H}$ & Proton \\
\hline AdMET & S-Adenosyl-L-methionine & $\mathrm{H} 2 \mathrm{O}$ & Water \\
\hline ADP & Adenosine diphosphate & $\mathrm{H} 2 \mathrm{O} 2$ & Hydrogen peroxide \\
\hline ADPG & Glucose Adenosine diphosphate & $\mathrm{H} 2 \mathrm{~S}$ & Sulphite \\
\hline AICAR & $\begin{array}{l}\text { 5-Aminoimidazole-4-carboxamide } \\
\text { ribonucleine }\end{array}$ & HCYS & Homocysteine \\
\hline AKG & $\alpha$-ketoglutarate & HIS & Histidine \\
\hline AKIV & 3-Methyl-2-oxobutanoic acid & HPYR & 3-Hydroxypyruvate \\
\hline ALA & Alanine & HSER & Homoserine \\
\hline AMP & Adenosine monophosphate & I3GLYCP & Indoleglycerol phosphate \\
\hline ANTH & Anthranilate & iClT & isocitrate \\
\hline APS & Adenylyl sulphate & ILE & Isoleucine \\
\hline ARG & Arginine & IMP & Inosine monophosphate \\
\hline ASA & L-Aspartate 4-semialdehyde & LEU & Leucine \\
\hline ASER & O-Acetyl-L-serine & Ipam & Enzyme N6-(lipoyl)lysine \\
\hline ASN & Asparagine & LYS & Lysine \\
\hline ASP & Aspartate & MAL & Malate \\
\hline ATP & Adenosine triphosphate & MalACP & Malonyl-ACP \\
\hline Biomass_x & Biomass & MalCoA & Malonyl-CoA \\
\hline C140ACP & Myristic acid ACP & Malt_dash_P & Maltose phosphate \\
\hline C160 & Palmitic acid & MET & Methionine \\
\hline C160ACP & Palmitic acid ACP & METHF & 5,10-Methylene-THF \\
\hline C160CoA & Palmitic acid CoA & Mg2 & Magnesium \\
\hline C161 & Palmitoleic acid & MGDG & $\begin{array}{l}\text { Monogalactosyl } \\
\text { diacylgycerol }\end{array}$ \\
\hline C161ACP & Palmitoleic acid ACP & MTHF & Methyl-THF \\
\hline C161CoA & Palmitoleic acid CoA & MYLTHF & 5,10-Methenyl-THF \\
\hline C162ACP & Hexadecadienoic ACP & N10FTHF & 10-Formyl-THF \\
\hline C162CoA & Hexadecadienoic acid CoA & NAD & Nicotinamide oxidised \\
\hline C163ACP & Hexadecatrienoic ACP & $\mathrm{NADH}$ & Nicotinamide reduced \\
\hline C180 & Stearic acid & NADP & $\begin{array}{l}\text { Nicotinamidephosphate } \\
\text { oxidised }\end{array}$ \\
\hline C180ACP & Stearic acid ACP & NADPH & $\begin{array}{l}\text { Nicotinamidephosphate } \\
\text { reduced }\end{array}$ \\
\hline C180CoA & Stearic acid CoA & $\mathrm{NH} 4$ & Ammonium \\
\hline C181 & Oleic acid & NO2 & Nitrite \\
\hline C181ACP & Oleic acid ACP & NO3 & Nitrate \\
\hline C181CoA & Oleic acid CoA & $\mathrm{O} 2$ & Oxygen \\
\hline C182ACP & Linoleic acid ACP & ORN & Ornithine \\
\hline C182CoA & Linoleic acid CoA & OXA & Oxaloacetate \\
\hline C183ACP & Linolenic acid ACP & P5C & $\begin{array}{l}\text { (S)-1-Pyrroline-5- } \\
\text { carboxylate }\end{array}$ \\
\hline C2_dash_oxobut & 2-Oxobutanoate & PEP & Phosphoenol pyruvate \\
\hline $\mathrm{CaP}$ & Carbomyl phosphate & PHE & Phenylalanine \\
\hline CARB & Carbohydrate & photon & Photon \\
\hline Carotene & $\beta$-carotene & PHPYR & Phenylpyruvate \\
\hline CDP & Cytidine diphosphate & Phytyl_dash_PP & Phytyl-diphosphate \\
\hline
\end{tabular}




\begin{tabular}{|c|c|c|c|}
\hline Chlorophyl & Chlorophyll a & $\mathrm{Pi}$ & Phosphate \\
\hline $\mathrm{CHO}$ & Chorismate & PPi & Diphosphate \\
\hline $\mathrm{CIT}$ & Citrate & PPorphyr & Protoporphyrine IX \\
\hline $\mathrm{CO} 2$ & Carbon dioxide & PRE & Prephenate \\
\hline CoA & Coenzyme A & PRO & Proline \\
\hline CTP & Cytosine triphosphate & PROTEIN & Protein \\
\hline CTU & L-Citrulline & PRPP & $\begin{array}{l}\text { Phosphoribosyl } \\
\text { pyrophosphate }\end{array}$ \\
\hline CYS & Cysteine & PYR & Pyruvate \\
\hline dADP & Deoxy ADP & R5P & Ribose 5-phosphate \\
\hline DAP & Diaminopimelate & RNA & Ribonucleic acid \\
\hline dATP & Deoxy ATP & RU15DP & Ribulose 1,5-bisphosphate \\
\hline $\mathrm{dCDP}$ & Deoxy CDP & RU5P & Ribulose 5-phosphate \\
\hline dCTP & Deoxy CTP & S7P & $\begin{array}{l}\text { Sedoheptulose 7- } \\
\text { phosphate }\end{array}$ \\
\hline dGDP & Deoxy GDP & SER & Serine \\
\hline dGTP & Deoxy GTP & SO3 & Sulphite \\
\hline DHAP & Dihydroxyacetone phosphate & SO4 & Sulphate \\
\hline DHF & Dihydrofolate & STARCH & Starch \\
\hline DHIV & 2,3-Dihydroxy-3-methylbutanoate & STARCH_dash_P & Starch phosphate \\
\hline DNA & Deoxiribonucleic acid & SUC & Succinate \\
\hline dTDP & Deoxy TDP & SUCCOA & Succinyl-CoA \\
\hline dTTP & Deoxy TTP & TAG & Triacylglycerol \\
\hline E4P & Erythrose 4-phosphate & THF & Tetrahydrofolate \\
\hline F16P & Fructose 1,6-biphosphate & thmpp & thiamin diphosphate \\
\hline F6P & Fructose 6-phosphate & THR & Threonine \\
\hline FAD & Flavine adenosine dinucleotide & trdox & Thioredoxin disulfide \\
\hline $\mathrm{FADH} 2$ & $\begin{array}{l}\text { Flavine adenosine dinucleotide } \\
\text { dihydride }\end{array}$ & trdrd & Thioredoxin \\
\hline fdxox & Ferredoxin oxidised & TRYP & Tryptophane \\
\hline fdxrd & Ferredoxin reduced & TYR & Tyrosine \\
\hline fluores & Fluorescent photon & UDP & Uridine diphosphate \\
\hline FORM & Formate & UDPGal & UDP-Galactose \\
\hline FUM & Fumarate & UMP & Uradine monophosphate \\
\hline G1P & Glucose 1-phosphate & UTP & Uracil triphosphate \\
\hline G6P & Glucose 6-phosphate & VAL & Valine \\
\hline GA & Glyceraldehyde & $\mathrm{X} 5 \mathrm{P}$ & Xylulose 5-phosphate \\
\hline GAP & Glyceraldehyde 3-phosphate & GDP & Guanosine diphosphate \\
\hline
\end{tabular}


Supplementary Table S6.1. Biomass equation coefficients under different specific growth rates. Where $\mu$ is the growth rate, $r_{E X}$ is the light supply rate, $\mathrm{CHO}$ is carbohydrates, $\mathrm{PL}$ is polar lipids, Pro is proteins, Chl is chlorophyll, and Car is carotene content.

\begin{tabular}{|c|c|c|c|c|c|c|c|c|}
\hline \multirow[t]{2}{*}{$\underset{\left(h^{-1}\right)}{\mu}$} & \multirow[t]{2}{*}{$\begin{array}{c}r_{E X} \\
\left(\mathrm{mmol}_{\mathrm{ph}} \cdot \mathrm{g}_{\mathrm{Dw}}{ }^{-1} \cdot \mathrm{h}^{-1}\right)\end{array}$} & \multicolumn{7}{|c|}{$\begin{array}{l}\text { Biomass equation coefficients } \\
\qquad\left(\mathrm{mmol} \cdot \mathrm{gDw}_{\mathrm{Dw}}\right)^{-1}\end{array}$} \\
\hline & & $\mathrm{CHO}$ & PL & Pro & Chl & Car & RNA & DNA \\
\hline 0.0290 & 23.0273 & 0.85 & 0.09 & 0.00 & 5.81 & 0.05 & 0.02 & 0.18 \\
\hline 0.0172 & 13.7583 & 0.91 & 0.09 & 0.00 & 5.68 & 0.05 & 0.02 & 0.18 \\
\hline 0.0115 & 9.3782 & 0.97 & 0.10 & 0.00 & 5.52 & 0.05 & 0.02 & 0.18 \\
\hline 0.0088 & 7.7120 & 1.07 & 0.09 & 0.00 & 5.49 & 0.04 & 0.01 & 0.18 \\
\hline 0.0076 & 6.3948 & 1.35 & 0.10 & 0.00 & 4.95 & 0.04 & 0.01 & 0.18 \\
\hline 0.0051 & 4.6098 & 1.45 & 0.10 & 0.00 & 4.79 & 0.04 & 0.01 & 0.18 \\
\hline 0.0040 & 3.7643 & 1.56 & 0.11 & 0.00 & 4.59 & 0.04 & 0.01 & 0.18 \\
\hline 0.0026 & 2.8801 & 1.54 & 0.11 & 0.00 & 4.55 & 0.04 & 0.01 & 0.18 \\
\hline 0.0016 & 2.5296 & 1.59 & 0.11 & 0.01 & 4.50 & 0.04 & 0.01 & 0.18 \\
\hline 0.0013 & 2.2697 & 1.63 & 0.10 & 0.01 & 4.54 & 0.03 & 0.01 & 0.18 \\
\hline 0.0018 & 2.1015 & 1.72 & 0.11 & 0.01 & 4.32 & 0.03 & 0.01 & 0.18 \\
\hline 0.0017 & 1.8502 & 1.70 & 0.10 & 0.01 & 4.36 & 0.04 & 0.01 & 0.18 \\
\hline 0.0000 & 1.6188 & 1.83 & 0.11 & 0.01 & 4.09 & 0.03 & 0.01 & 0.18 \\
\hline 0.0473 & 53.3250 & 0.62 & 0.08 & 0.01 & 6.10 & 0.04 & 0.01 & 0.18 \\
\hline 0.0296 & 42.2138 & 0.66 & 0.08 & 0.00 & 6.16 & 0.04 & 0.01 & 0.18 \\
\hline 0.0309 & 38.1589 & 0.75 & 0.08 & 0.00 & 6.01 & 0.04 & 0.01 & 0.18 \\
\hline 0.0217 & 27.2485 & 0.77 & 0.08 & 0.01 & 5.91 & 0.05 & 0.01 & 0.18 \\
\hline 0.0197 & 19.1209 & 0.74 & 0.09 & 0.01 & 5.88 & 0.04 & 0.01 & 0.18 \\
\hline 0.0146 & 12.9069 & 0.92 & 0.08 & 0.01 & 5.53 & 0.04 & 0.01 & 0.18 \\
\hline 0.0097 & 9.9252 & 0.78 & 0.08 & 0.01 & 5.90 & 0.04 & 0.01 & 0.18 \\
\hline 0.0080 & 8.1479 & 0.79 & 0.08 & 0.00 & 5.93 & 0.03 & 0.01 & 0.18 \\
\hline 0.0068 & 6.5758 & 0.89 & 0.08 & 0.00 & 5.72 & 0.04 & 0.01 & 0.18 \\
\hline 0.0148 & 13.5818 & 0.71 & 0.08 & 0.00 & 5.96 & 0.04 & 0.01 & 0.18 \\
\hline 0.0085 & 10.1829 & 0.67 & 0.08 & 0.00 & 6.00 & 0.04 & 0.01 & 0.18 \\
\hline 0.0067 & 8.9115 & 0.64 & 0.08 & 0.00 & 6.19 & 0.04 & 0.01 & 0.18 \\
\hline 0.0528 & 51.0985 & 0.78 & 0.08 & 0.00 & 6.02 & 0.04 & 0.01 & 0.18 \\
\hline 0.0344 & 32.7041 & 0.75 & 0.08 & 0.00 & 5.90 & 0.05 & 0.02 & 0.18 \\
\hline 0.0225 & 18.8957 & 0.72 & 0.10 & 0.00 & 5.83 & 0.04 & 0.01 & 0.18 \\
\hline 0.0137 & 12.3185 & 0.90 & 0.08 & 0.00 & 5.69 & 0.04 & 0.01 & 0.18 \\
\hline 0.0045 & 4.7383 & 1.32 & 0.11 & 0.01 & 4.43 & 0.05 & 0.02 & 0.18 \\
\hline 0.0027 & 3.2243 & 1.14 & 0.12 & 0.00 & 4.95 & 0.04 & 0.02 & 0.18 \\
\hline 0.0021 & 2.5327 & 1.18 & 0.12 & 0.01 & 4.99 & 0.05 & 0.02 & 0.18 \\
\hline 0.0014 & 2.0437 & 1.16 & 0.12 & 0.00 & 5.06 & 0.05 & 0.02 & 0.18 \\
\hline 0.0007 & 1.8469 & 1.16 & 0.11 & 0.00 & 5.04 & 0.05 & 0.02 & 0.18 \\
\hline 0.0005 & 1.7709 & 1.28 & 0.12 & 0.01 & 4.81 & 0.05 & 0.02 & 0.18 \\
\hline 0.0000 & 1.6125 & 1.07 & 0.14 & 0.01 & 5.19 & 0.04 & 0.02 & 0.18 \\
\hline 0.0000 & 1.6338 & 1.09 & 0.13 & 0.01 & 5.16 & 0.05 & 0.02 & 0.18 \\
\hline 0.0000 & 1.6718 & 1.11 & 0.12 & 0.01 & 5.08 & 0.05 & 0.02 & 0.18 \\
\hline 0.0000 & 1.6346 & 1.12 & 0.13 & 0.01 & 5.02 & 0.05 & 0.02 & 0.18 \\
\hline 0.0043 & 5.1123 & 1.31 & 0.11 & 0.00 & 4.64 & 0.04 & 0.01 & 0.18 \\
\hline 0.0030 & 3.7526 & 1.44 & 0.12 & 0.00 & 4.38 & 0.04 & 0.02 & 0.18 \\
\hline 0.0020 & 2.7097 & 1.60 & 0.12 & 0.01 & 4.25 & 0.04 & 0.02 & 0.18 \\
\hline
\end{tabular}




\begin{tabular}{|lllllllll}
\hline 0.0010 & 2.3263 & 1.83 & 0.11 & 0.01 & 4.09 & 0.04 & 0.02 & 0.18 \\
\hline 0.0004 & 2.2094 & 1.82 & 0.12 & 0.02 & 4.05 & 0.04 & 0.02 & 0.18 \\
\hline 0.0005 & 2.1004 & 1.61 & 0.12 & 0.02 & 4.44 & 0.03 & 0.01 & 0.18 \\
\hline 0.0000 & 2.1458 & 1.61 & 0.13 & 0.02 & 4.35 & 0.03 & 0.01 & 0.18 \\
\hline 0.0000 & 2.1153 & 1.52 & 0.14 & 0.02 & 4.41 & 0.03 & 0.01 & 0.18 \\
\hline 0.0000 & 2.0887 & 1.55 & 0.14 & 0.02 & 4.34 & 0.04 & 0.01 & 0.18 \\
\hline 0.0041 & 4.7519 & 1.21 & 0.11 & 0.00 & 4.64 & 0.04 & 0.01 & 0.18 \\
\hline 0.0016 & 3.5357 & 1.31 & 0.12 & 0.00 & 4.66 & 0.04 & 0.02 & 0.18 \\
\hline 0.0016 & 3.2059 & 1.21 & 0.11 & 0.00 & 4.90 & 0.04 & 0.02 & 0.18 \\
\hline 0.0016 & 2.5397 & 1.41 & 0.11 & 0.00 & 4.69 & 0.04 & 0.02 & 0.18 \\
\hline 0.0009 & 2.1122 & 1.63 & 0.12 & 0.01 & 4.35 & 0.04 & 0.01 & 0.18 \\
\hline 0.0006 & 1.9879 & 1.67 & 0.12 & 0.01 & 4.42 & 0.04 & 0.02 & 0.18 \\
\hline 0.0001 & 1.8544 & 1.55 & 0.12 & 0.02 & 4.52 & 0.03 & 0.01 & 0.18 \\
\hline 0.0000 & 1.8727 & 1.53 & 0.13 & 0.01 & 4.55 & 0.03 & 0.01 & 0.18 \\
\hline 0.0000 & 1.9073 & 1.73 & 0.12 & 0.01 & 4.19 & 0.04 & 0.01 & 0.18 \\
\hline 0.0000 & 1.8677 & 1.63 & 0.14 & 0.02 & 4.23 & 0.03 & 0.01 & 0.18 \\
\hline 0.0000 & 1.8122 & 1.59 & 0.12 & 0.01 & 4.40 & 0.03 & 0.01 & 0.18 \\
\hline 0.0049 & 4.9241 & 1.26 & 0.12 & 0.00 & 4.57 & 0.04 & 0.02 & 0.18 \\
\hline 0.0031 & 3.1699 & 1.38 & 0.12 & 0.01 & 4.64 & 0.04 & 0.02 & 0.18 \\
\hline 0.0019 & 2.4425 & 1.53 & 0.11 & 0.01 & 4.37 & 0.04 & 0.02 & 0.18 \\
\hline 0.0008 & 2.1411 & 1.47 & 0.10 & 0.01 & 4.76 & 0.04 & 0.02 & 0.18 \\
\hline 0.0008 & 2.0004 & 1.48 & 0.11 & 0.01 & 4.62 & 0.04 & 0.02 & 0.18 \\
\hline 0.0007 & 1.8206 & 1.47 & 0.13 & 0.01 & 4.67 & 0.04 & 0.01 & 0.18 \\
\hline 0.0009 & 1.7081 & 1.65 & 0.10 & 0.01 & 4.51 & 0.03 & 0.01 & 0.18 \\
\hline 0.0000 & 1.6550 & 1.80 & 0.11 & 0.02 & 4.16 & 0.04 & 0.01 & 0.18 \\
\hline 0.0000 & 1.6995 & 1.88 & 0.10 & 0.02 & 4.05 & 0.04 & 0.01 & 0.18 \\
\hline 0.0000 & 1.6442 & 1.65 & 0.09 & 0.01 & 4.55 & 0.04 & 0.01 & 0.18 \\
\hline & & & & & & & & \\
\hline
\end{tabular}

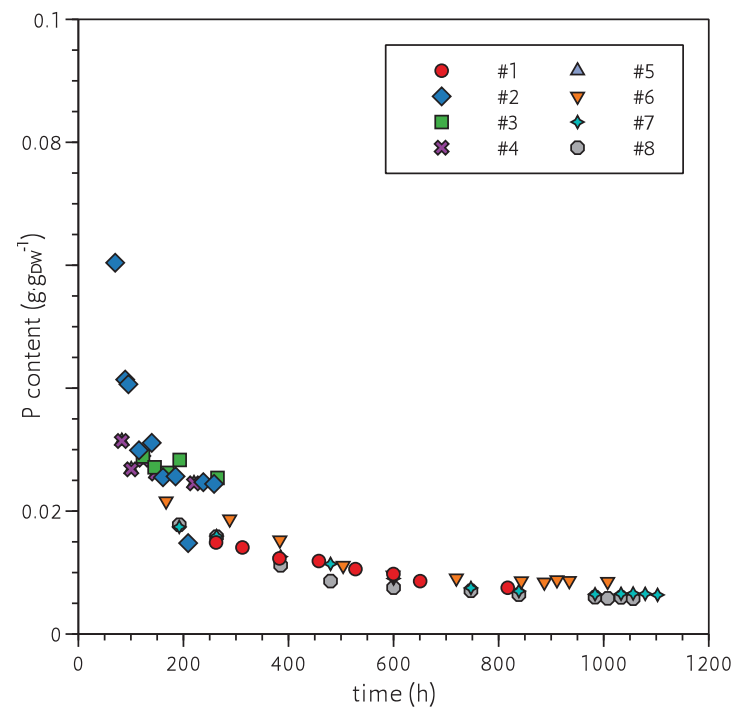

Supplementary Figure S6.1. Phosphorus content calculated based on uptake and biomass concentration during eight different batch cultures of Tetradesmus obliquus. 


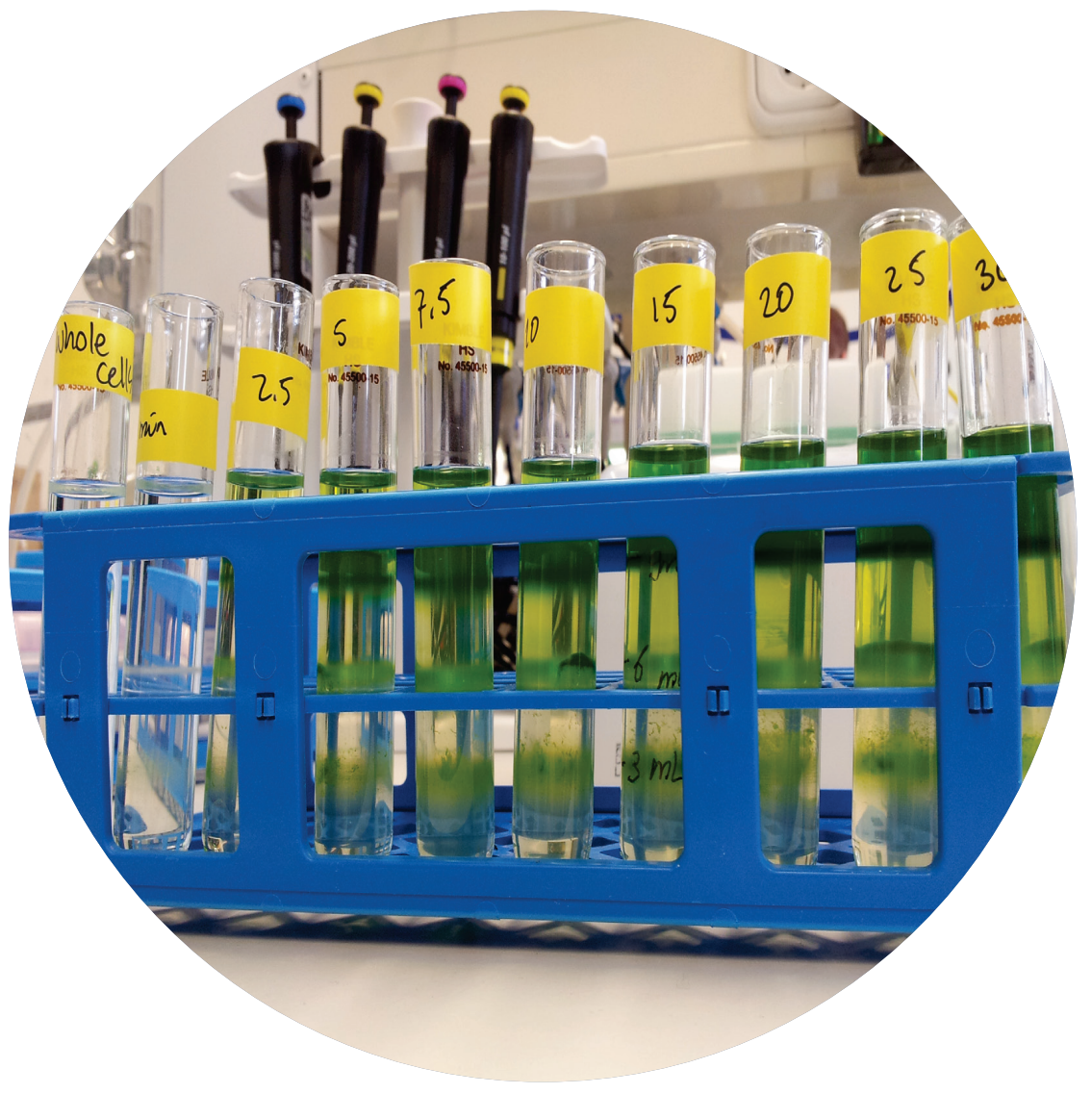




\section{Chapter}

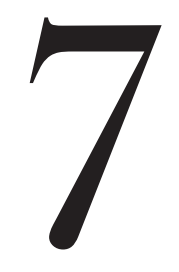

General discussion 
Microalgal large scale production of lipids is commonly divided in two phases: the growth phase and the lipid (triacylglycerides, TAG) production phase. During the growth phase, favourable nitrogen replete conditions and light/dark (LD) cycles are used. In this phase, microalgae synchronize their metabolism to anticipate the changing environment. This results in changes in starch content during the diurnal cycle, where starch is used during the dark period and the first $4 \mathrm{~h}$ of the light period and accumulated during the rest of the light period. Since starch competes for carbon precursors that could otherwise be directed towards TAG, a strategy to increase TAG production is to block starch production. The inability to produce starch results in higher production of TAG during nitrogen starvation (lipid production phase), but has a negative effect on the photosynthetic efficiency under nitrogen replete conditions (growth phase).

This chapter discusses the role and importance of starch in oleaginous microalgae. We found that the role of starch depends on the environmental conditions. Under nitrogen replete conditions, starch serves as a diurnal energy storage compound that can be easily used during the dark period to provide energy and carbon. Starch is also consumed at the beginning of the light period, possibly to provide extra energy to rapidly produce pigments and harvest light energy. Under nitrogen limitation, starch still functions as a diurnal energy storage compound, but to a lower extent. Finally, under nitrogen starvation, starch is still the primary storage compound for energy and carbon in $T$. obliquus, but it is no longer used as a diurnal energy storage compound under this condition. Instead, starch serves as a storage compound to be used when nitrogen becomes available again and possibly also as an electron sink. TAG accumulation mainly occurs once the storage capacity for starch is full $\left(0.40 \mathrm{~g} \cdot \mathrm{gDw}^{-1}\right)$. On longer terms, when nitrogen remains depleted, the starch is also converted to TAG and TAG serves as a long term storage compound, to be used for recovery when nitrogen becomes available again. 


\subsection{Introduction}

Microalgae are promising sustainable feedstocks for the production of fuels, chemicals, food, and feed, as they combine the advantages of high growth rate and high photosynthetic efficiency (Brányiková et al., 2011; Klok et al., 2014; Ruiz et al., 2016). However, according to a study performed by Ruiz et al. (2016), only high-value products (such as pigments) are currently economically feasible and bulk commodities (such as biofuels and chemicals) still require cost reductions to make them profitable. Microalgae are of special interest due to their high lipid content. Microalgae that produce large amounts of triacylglycerides (TAG) (between $20-60 \%$ of TAG on a dry weight (DW) basis under stress conditions) are known as oleaginous microalgae (Hu et al., 2008). A promising oleaginous microalgae is Tetradesmus obliquus. This microalga can reach a TAG content of up to $0.45 \mathrm{~g} \cdot \mathrm{gDw}^{-1}$ and a maximum TAG yield on light of $0.14 \mathrm{~g} \cdot \mathrm{mol}_{\mathrm{Ph}^{-1}}{ }^{-1}$ under batch nitrogen starvation (Breuer et al., 2014).

Many microalgae synchronize their metabolism to light/dark (LD) cycles and accumulate starch. Starch is produced and stored during the light period and used during the night to provide carbon and energy. When aiming for TAG accumulation, starch is an unwanted product. This is because starch and TAG are competing for carbon and energy as they share common C3 precursors in the carbon metabolism (Box 7.1). Therefore, a strategy to increase TAG content in microalgae is to develop starchless mutants by blocking starch production. This strategy has been successful in microalgae that accumulate starch as a storage compound, such as Chlamydomonas reinhardtii (Li et al., 2010a), Chlorella pyrenoidosa (Ramazanov and Ramazanov, 2006), and Tetradesmus obliquus (de Jaeger et al., 2014). The starchless mutant $\operatorname{sim} 1$ of T. obliquus reached a higher TAG content $\left(0.57 \mathrm{~g} \cdot \mathrm{gDW}^{-1}\right.$ compared to $0.45 \mathrm{~g} \cdot \mathrm{gDW}^{-1}$ for the wild-type) and a higher maximum TAG yield on light $\left(0.22 \mathrm{~g} \cdot \mathrm{mol}_{\mathrm{Ph}}{ }^{-1}\right.$ compared to $0.14 \mathrm{~g} \cdot \mathrm{molph}^{-1}$ for the wild-type) under batch nitrogen starvation (Breuer et al., 2014).

Additionally, not all oleaginous microalgae rely on starch to store energy during the diurnal cycles. Microalgae species belonging to the Nannochloropsis genus accumulate lipids during nitrogen replete conditions. Under LD cycles, lipids and carbohydrates act as diurnal energy storage compounds in the microalga $N$. gaditana (Fábregas et al., 2002). In N. oceanica grown under batch cultivation and 12:12 h LD cycles, TAG was 
the only lipid that showed oscillations (fatty acids content from approximately $10.5 \%$ DW at the beginning of the dark period to around 9.5\% DW at the beginning of the light period), suggesting a role of TAG in transitory carbon storage in this microalga (Poliner et al., 2015). Additionally, total glucose content also oscillated under diurnal LD cycles (from approximately $3.1 \%$ DW at the beginning of the dark period to $1.8 \%$ DW at the beginning of the light period). Poliner et al. (2015) attributed the decrease in glucose to shedding of the cell wall during cell division (cellulose) and the use of glucose-containing storage compounds (laminarin).

\section{Box 7.1 Starch and triacylglycerides (TAG) metabolism}

Photosynthetic organisms convert $\mathrm{CO}_{2}$ into organic compounds using light energy. The fixated carbon is partly stored in storage molecules (such as starch or TAG) that can be broken down to provide ATP, reducing power (NADPH), and carbon precursors to the cell (Johnson and Alric, 2013; Jia et al., 2015).

Starch and TAG are competing for carbon as they share common C3 precursors in the carbon metabolism (such as 3-phosphoglycerate, 3PG, and glyceraldehyde 3phosphate, GAP), as shown in the simplified schematic view of TAG and starch metabolism (Figure 7.1).

Starch is normally the storage molecule synthesized and degraded during light/dark cycles. For starch biosynthesis, the first step is the synthesis of ADP Glucose via the ADP Glucose pyrophosphorylase. Next, the Granule Bond Starch Synthase (GBSS) catalyses the elongation of $\alpha-1,4$-glucans by transferring the glucosyl part from the sugar nucleotide to the non-reducing end of the growing polyglucan chain. Amylopectin synthesis involves soluble starch synthases while amylose synthesis involves the GBSSs. In green algae, starch is accumulated in the plastids (Johnson and Alric, 2012; Busi et al., 2014).

De novo lipid biosynthesis is known to occur in two locations: the chloroplast and the endoplasmic reticulum (ER) membranes (de Jaeger et al., 2014; Klok et al., 2014) (Figure 7.1). In the chloroplast, fatty acids are synthesized from acetyl-CoA. First, acetyl-CoA is converted to malonyl-CoA (Mal-CoA) via the enzyme acetyl-CoA carboxylase. Subsequently, the Mal-CoA is transferred to an acyl carrier protein 
(ACP) after which the multi-subunit fatty acid synthase complex extends the malonyl-ACP by two carbon atoms per cycle. Once the fatty-acyl chain reaches the appropriate length, the chain is removed from the ACP group and transferred to glycerol-3-phosphate to produce TAG or membrane lipids (Stern, 2009; Klok et al., 2014). In the ER pathway, free fatty acids are transported from the chloroplast to the ER to be attached to CoA by the enzyme long-chain acyl-CoA synthase. After this step the pathway is the same as in the chloroplast, using acyl-CoA as a substrate instead of acyl-ACP (Klok et al., 2014).

Under prolonged nitrogen starvation, microalgae have been shown to degrade starch to most likely convert it into TAG (Breuer et al., 2014).

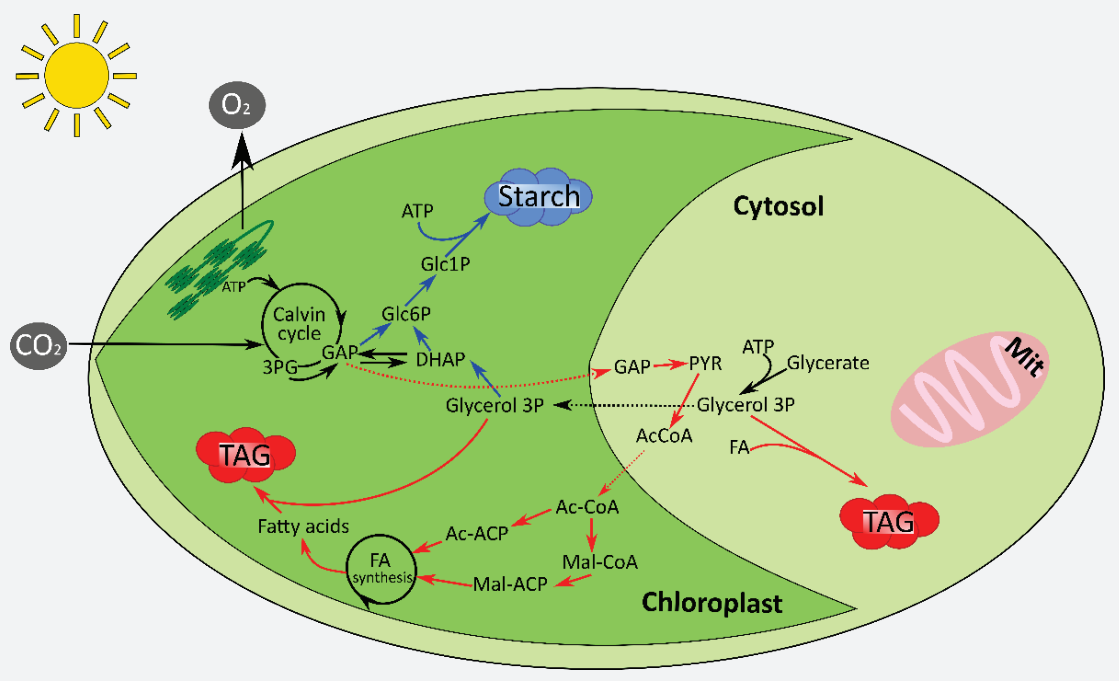

Figure 7.1. Simplified overview of starch and triacylglycerides (TAG) metabolism in Tetradesmus obliquus. Not all reactions and cofactors are shown. Blue arrows indicate flow towards starch and red arrows towards TAG. Dashed lines indicate transport reactions between the organelles. 3PG: 3-Phosphoglycerate; GAP: glyceraldehyde 3-phosphate; PYR: pyruvate; DHAP: Dihydroxyacetone phosphate; Glc6P: glucose 6-phosphate; Glc1P: glucose 1-phosphate; Ac-CoA: Acetyl-CoA; Mal-CoA: Malonyl-CoA; ACP: Acyl Carrier Protein; Mit: mitochondrion. 
Since starch is accumulated during the light period to be used as a carbon and energy source during dark periods, growth and energy efficiency of the starchless mutant was expected to be the same as for the wild-type under continuous light. This was, however, not the case for $T$. obliquus, as the mutant $\operatorname{s} / m 1$ showed a lower growth rate and biomass yield on light under continuous light (Chapter 2). Nevertheless, under nitrogen starvation, the starchless mutant $\operatorname{s} / m 1$ was indeed equally efficient as the wild-type under both continuous light (Breuer et al., 2014) and LD cycles (Remmers et al., 2017). These results made clear that starch has different functions depending on the environmental conditions and that during replete conditions the benefit is not only in supplying energy during the night. In this chapter, the role of starch in oleaginous microalgae under both nitrogen replete and deplete conditions is discussed.

\subsection{Role of starch and TAG in oleaginous algae under nitrogen replete conditions}

Microalgal large scale production must be done outdoors, where microalgae are exposed to diurnal light/dark (LD) cycles. Under LD cycles and nitrogen replete conditions, T. obliquus showed diurnal oscillations in starch content (Chapter 2). Starch thus served as the diurnal energy storage compound: it was accumulated during the final part of the light period (5-16 $\mathrm{h}$ after the light went on) and was used during the dark period (16-23 h) and early light period (0-4 h) (Figure 7.2A). These results are in agreement with the transcriptome data (Chapter 3), but to a lesser extent. During the dark period, starch is probably used to support cell division, DNA replication, and nitrogen assimilation (Figure 7.3A), allowing them to complete the cell cycle without any external supply of carbon and energy (Vitova et al., 2015). In Figure 7.3 it can be observed that whereas the wild-type consumes nitrate during the night the starchless mutant does not do this. This indicates that starch is needed to assimilate the nitrogen, by supplying the needed reducing equivalents and/or the carbon to eventually bind the nitrogen in amino acids. The usage of starch at night is also supported by the transcriptome data from Chapter 3: DNA replication seems to occur during the lateday or early-night, cell division occurs during the night, as is the case for nitrogen metabolism and protein synthesis. Autotrophically growing algae are known to also use their storage reserves even when photosynthesis is active (Vítová et al., 2011). This was observed for T. obliquus wild-type, where starch was not fully consumed during 
the dark period, and consumption continued when light was again available (Chapters 2 and 4). Starch consumption during the first $4 \mathrm{~h}$ of the light period is concomitant with a steep increase in dilution rate during our turbidostat controlled experiments, which is caused by a rapid increase in pigment production. Therefore starch might provide energy to rapidly produce pigments at the beginning of the day, after cell division is completed, allowing the algae to more rapidly harvest light. The rapid increase in pigment production at the beginning of the day is also supported by the transcriptome data in Chapter 3, where pigment synthesis is steeply upregulated at the beginning of the day.

The presence of starch is not needed for cell division at night, as the starchless mutant slm1 is also able to divide during the dark period (Chapter 4) without a clear internal source of energy or carbon. However, starch contributes to cell division in the wildtype, as cell division was faster in the wild-type compared to the $\operatorname{sim} 1$.

When testing for the effect of different day lengths (or photoperiods), variations were observed in starch content (Chapter 4). The longer the light period, the higher the starch content reached $\left(0.16 \mathrm{~g} \cdot \mathrm{g}_{\mathrm{DW}}{ }^{-1}\right.$ for the $12: 12 \mathrm{~h} \mathrm{LD}, 0.17 \mathrm{~g} \cdot \mathrm{g}_{\mathrm{DW}}{ }^{-1}$ for the 14:10 $\mathrm{h} \mathrm{LD}$, and $0.22 \mathrm{~g} \cdot \mathrm{gDw}^{-1}$ for the $16: 8 \mathrm{~h}$ LD). Additionally, starch was not fully consumed during the dark period and a fraction remained (0.03-0.06 g.g $\left.\mathrm{DW}^{-1}\right)$. This indicates that only a certain amount of energy is needed during the dark period (70-80\% of the reserve), independently of the length of the dark period.

We then looked at other oleaginous microalgae that produces both starch and TAG as storage compounds and estimated the energy supplied by their storage compounds (the yield from the complete oxidation of fatty acids is $38 \mathrm{~kJ} \cdot \mathrm{g}^{-1}$ and for carbohydrates is $17 \mathrm{~kJ} \cdot \mathrm{g}^{-1}$ (Berg et al., 2002)). For the microalga Neochloris oleoabundans, grown in turbidostat mode and $500 \mu \mathrm{mol} \cdot \mathrm{m}^{-2} \cdot \mathrm{s}^{-1}$ (based on the results presented by de Winter et al. (2017b)), the energy supplied by starch consumption during the night is comparable to the one of T. obliquus under $16: 8 \mathrm{~h}$ LD cycles $\left(2.63 \mathrm{KJ} \cdot \mathrm{gDw}^{-1}\right.$ for T. obliquus compared to $2.55 \mathrm{KJ} \cdot \mathrm{gDw}^{-1}$ for $N$. oleoabundans). Under $12: 12 \mathrm{~h} \mathrm{LD}$, the value estimated for $N$. oleoabundans $\left(1.36 \mathrm{KJ} \cdot \mathrm{g}_{\mathrm{DW}}{ }^{-1}\right)$ is lower than for T. obliquus $\left(2.14 \mathrm{KJ} \cdot \mathrm{gDw}^{-1}\right)$. However, for $N$. oleoabundans the last measured value of starch content was $2 \mathrm{~h}$ before the beginning of the dark period. Assuming that the starch content would still increase approximately 4\% DW (approximately from 12\% to 16\% 
DW), the energy used during the night would be comparable to the one used by T. obliquus.

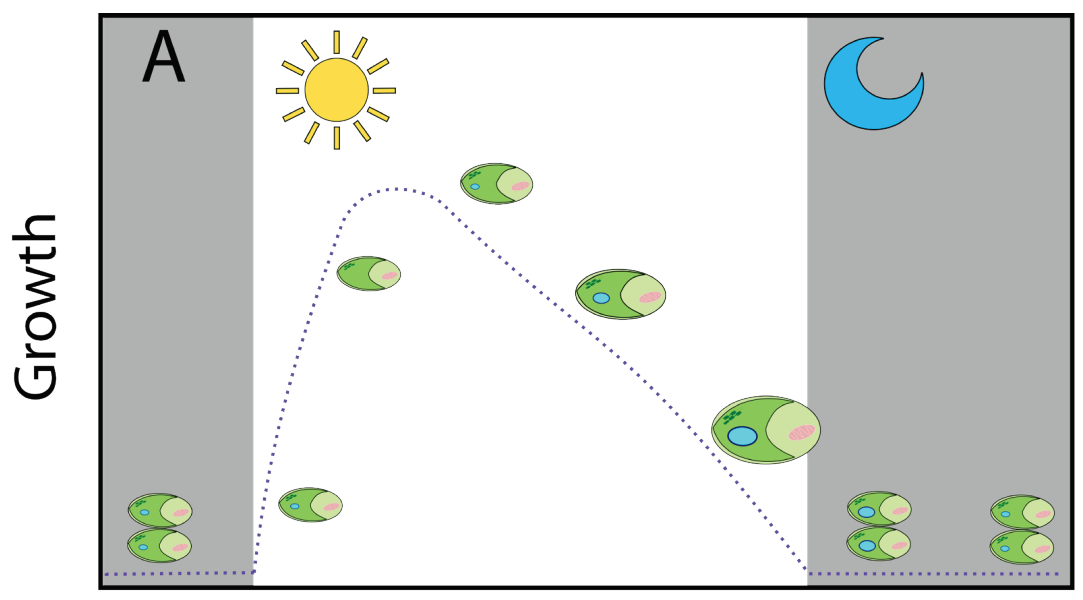

Time

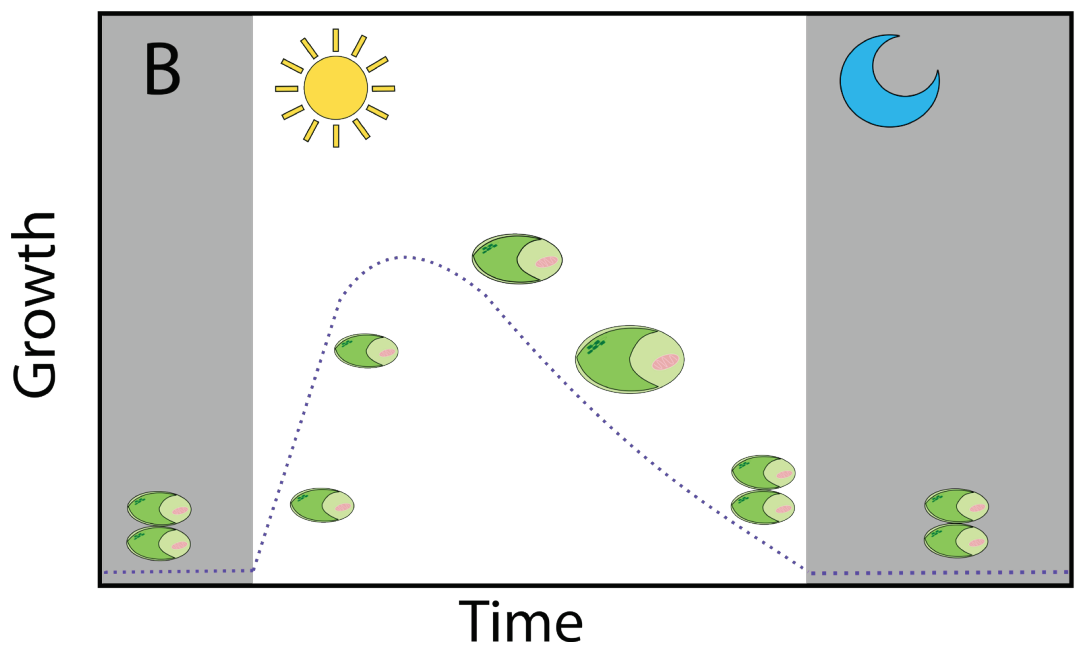

Figure 7.2. Representation of oscillations in cell size and starch content during light/dark cycles for Tetradesmus obliquus wild-type (A) and slm1 (B). Blue circle inside the cells represent the starch content. Dashed line indicates the dilution rate (growth rate) in a photobioreactor operated as turbidostat. Shaded area indicates the dark period. 

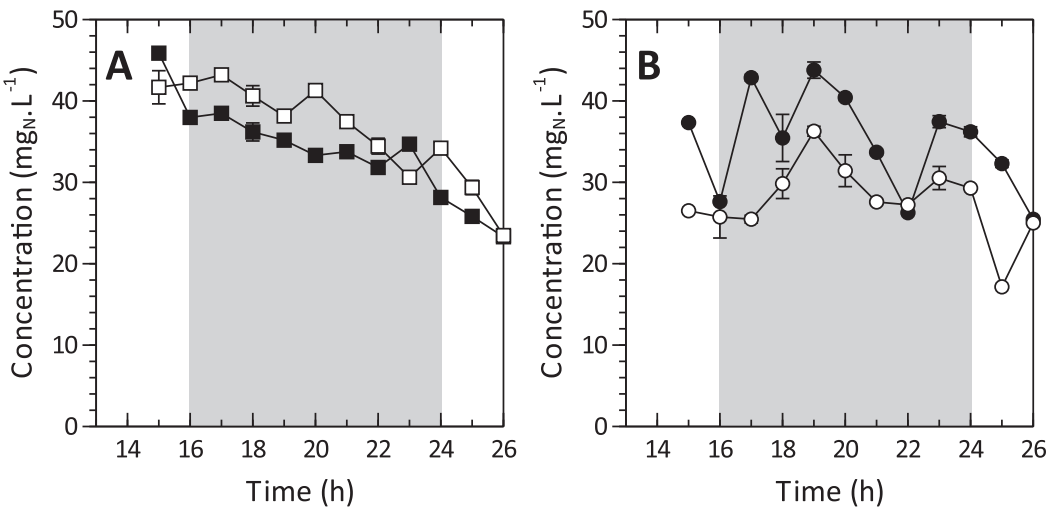

Figure 7.3. Nitrogen concentration in supernatant samples of Tetradesmus obliquus wild-type (A) and $s / m 1$ (B) under 16:8 h light/dark cycles. Open and closed symbols represent biological duplicates. The $x$ axis represents hours after 'sunrise'. Shaded area indicates the dark period. Error bars in nitrogen measurements represent the deviation in technical replicates $(n \geq 2)$.

As stated before, N. oceanica uses TAG as a diurnal storage compound. TAG is a reduced and anhydrous compound that can, therefore, concentrate a higher amount of energy compared to carbohydrates. By calculating the overnight differences in storage compounds for $N$. oceanica based on the results presented by Poliner et al. (2015) we estimated that the energy supplied by the oxidation of both fatty acids and glucose represents approximately $28 \%$ of the energy used by $T$. obliquus through the breakdown of starch overnight (under 12:12 h LD T. obliquus obtains $2.14 \mathrm{KJ} \cdot \mathrm{gDw}^{-1}$ compared to $0.60 \mathrm{KJ} \cdot \mathrm{gDW}^{-1}$ for $N$. oceanica). The difference can be explained by a lower growth rate for $N$. oceanica, the fact that they are a different microalgae genus, and/or the different culture conditions. The growth rate was about 3 times lower than the one for $T$. obliquus (approximately 0.30 day $^{-1}$ compared to 0.87 day $^{-1}$ ). Furthermore, the cultivation conditions were different: T. obliquus was grown under continuous cultivation and $500 \mu \mathrm{mol} \cdot \mathrm{m}^{-2} \cdot \mathrm{s}^{-1}$ while $N$. oceanica was grown in flasks under batch conditions and $40 \mu \mathrm{mol} \cdot \mathrm{m}^{-2} \cdot \mathrm{s}^{-1}$.

When looking at the impact of blocking starch production, as in the starchless mutant s/m1, TAG nor any other compound took over the role of starch as diurnal energy storage compound (Chapter 2). This translated into lower growth during the whole diurnal cycle and changes in cell division time for the $\operatorname{sim} 1$ compared to the wild-type 
(as represented in Figure 7.2B) (Chapter 4). Additionally, as previously stated, no nitrogen consumption was observed for the $\operatorname{sim} 1$ mutant at night (Figure 7.3B), indicating that starch is involved with this process. Also in the transcriptome data (Chapter 3) a shift of gene expression profiles in time was observed. However the link with the absence of starch and the observed differences in growth and division was not so clear. The starchless mutant $s / m 1$ also showed an increase in growth rate during the first hours of light; however, the wild-type reached a higher maximum growth rate, possibly due to starch providing extra energy during those first hours of light or to the fact that nitrogen is taken up during the night. This means that when the light is on, the wild-type already fixated nitrogen during the night and prepared for the next day, while the $\operatorname{sim} 1$ has to do this at the beginning of the day. Overall, the lack of starch results in lower biomass productivity and lower biomass yield on light under all the tested photoperiods (Table 7.1).

Table 7.1. Average steady state values of biomass productivity $\left(r_{x}\right)$ and biomass yield on light $\left(\mathrm{Y}_{\mathrm{x} / \mathrm{ph}}\right)$ for Tetradesmus obliquus wild-type and $s / m 1$ under different light/dark (LD) cycles. Values indicate the average \pm standard deviation.

\begin{tabular}{|c|c|c|c|c|c|}
\hline \multirow{2}{*}{$\begin{array}{l}\text { Strain } \\
\text { LD cycle }\end{array}$} & \multicolumn{2}{|c|}{ Wild-type } & \multicolumn{2}{|c|}{$s / m 1$} & \multirow[b]{2}{*}{ Reference } \\
\hline & $\begin{array}{c}r_{x} \\
\left(g_{D w} \cdot L^{-1} \cdot d_{a y}^{-1}\right)\end{array}$ & $\begin{array}{c}\mathrm{Y}_{\mathrm{x} / \mathrm{ph}} \\
\left(\mathrm{g}_{\mathrm{Dw}} \cdot \mathrm{mol}_{\mathrm{ph}}{ }^{-1}\right)\end{array}$ & $\begin{array}{c}r_{x} \\
\left(g_{D w} \cdot L^{-1} \cdot d^{-1}\right)\end{array}$ & $\begin{array}{c}\mathrm{Y}_{\mathrm{x} / \mathrm{ph}} \\
\left(\mathrm{g}_{\mathrm{DW}} \cdot \mathrm{mol}_{\mathrm{ph}}{ }^{-1}\right)\end{array}$ & \\
\hline $24: 0$ & $2.02 \pm 0.16$ & $0.98 \pm 0.08$ & $1.63 \pm 0.08$ & $0.80 \pm 0.04$ & Chapter 2 \\
\hline $16: 8$ & $1.61 \pm 0.04$ & $1.18 \pm 0.03$ & $0.97 \pm 0.06$ & $0.72 \pm 0.04$ & Chapter 2 \\
\hline $14: 10$ & $1.08 \pm 0.09$ & $0.91 \pm 0.07$ & $0.94 \pm 0.00$ & $0.79 \pm 0.00$ & Chapter 4 \\
\hline $12: 12$ & $0.93 \pm 0.03$ & $0.91 \pm 0.03$ & $0.70 \pm 0.03$ & $0.68 \pm 0.03$ & Chapter 4 \\
\hline
\end{tabular}

Results suggest that starch plays a role beyond the supply of energy at night, otherwise both strains would have maintained the same behaviour under continuous light. One explanation for this would be that, as the starchless mutant $\operatorname{sim} 1$ was created by random mutagenesis, the variations on energy efficiency and cell division could be related to other mutations that were introduced additionally to the one in the ADP glucose pyrophosphorylase. To verify that the mutation only changed the ability to synthesize starch, the starch synthesis capability should be restored in s/m1 and this revertant strain should again behave as the wild-type. Another possible explanation is that growth has not been optimized for the mutant, as has been done for the wildtype. However, most likely the differences in behaviour are related to the function of 
starch. Certainly, looking over a whole day the ability to produce starch allow microalgae to harvest extra light energy under LD cycles, which is not possible anymore under continuous light. Under continuous light synchronization is lost and cell division occurs randomly (de Winter, 2015). During this "unsynchronized" growth, some algae cells might still go through a period during cell division in which the cells cannot use light and they rely on starch. This would mean that under continuous light for individual cells the starch content is still fluctuating, but the overall change cannot be observed in the analysis. During this period of starch use, the light supplied is used less efficiently, resulting thus in an overall loss of photosynthetic efficiency.

\subsection{Role of starch and TAG in oleaginous algae under nitrogen deplete conditions}

Under stress conditions (nitrogen deplete), the metabolism of microalgae changes. Many microalgae respond to these conditions with the synthesis of triacylglycerides (TAG), as this serves not only as carbon and energy storage, but the de novo synthesis of TAG also serves as an electron sink under photo-oxidative stress to prevent formation of reactive oxygen species. Under stress, over-production of reactive oxygen species occurs due to the excess of electrons, causing inhibition of photosynthesis and damage to membrane lipids, proteins and other macromolecules (Hu et al., 2008). The stored compounds can also be used by the microalgae to recover from nitrogen starvation after the resupply of nitrogen, as seen in Chromochloris zofingiensis and Parachlorella kessleri, by, for example, supplying carbon (precursors) and energy (Fernandes et al., 2013; Mulders et al., 2014).

TAG production is commonly done using a batch nitrogen starvation process (Rodolfi et al., 2009; Breuer et al., 2015b; Benvenuti et al., 2016; Remmers et al., 2017) as this process allows TAG contents of up to $60 \%$ on a dry weight basis (Breuer et al., 2012). Some microalgae, such as Chlamydomonas reinhardtii, are known to accumulate large quantities of starch under stress conditions (Ball et al., 1990; Brányiková et al., 2011). While other microalgae, such as Tetradesmus obliquus, accumulate starch and TAG. Starch was the preferred storage compound and TAG is mainly formed once the maximum storage capacity of starch $\left(0.40 \mathrm{~g} \cdot \mathrm{g}_{\mathrm{DW}}{ }^{-1}\right)$ was reached, and the carbon and energy supply continued (Remmers et al., 2017). When blocking starch production, 
TAG accumulation starts immediately after nitrogen starvation and starchless mutants have a better carbon partitioning towards TAG (Ramazanov and Ramazanov, 2006; Li et al., 2010a; Breuer et al., 2014).

Therefore, during the lipid production phase, the starchless mutant $s / m 1$ offers the advantage of accumulating TAG directly during the first period of stress. While in the wild-type part of the carbon is stored as starch at the beginning and, therefore, the TAG productivity is lower. The maximum TAG content is higher in the $\operatorname{sim} 1\left(0.57 \mathrm{~g} \cdot \mathrm{gDW}^{-1}\right.$ compared to $\left.0.45 \mathrm{~g} \cdot \mathrm{gDw}_{\mathrm{DW}}^{-1}\right)$. With a higher maximum TAG yield on light for the $\operatorname{sim} 1$ $\left(0.20 \mathrm{~g}_{\mathrm{TAG}} \cdot \mathrm{mol}_{\mathrm{ph}}{ }^{-1}\right.$ for the $\mathrm{s} / \mathrm{m} 1$ compared to $0.16 \mathrm{~g}_{\mathrm{TAG}} \cdot \mathrm{mol}_{\mathrm{ph}}{ }^{-1}$ for the wild-type), corresponding to volumetric TAG productivities of $0.29 \mathrm{~g} \cdot \mathrm{L}^{-1} \mathrm{day}^{-1}$ for the $\operatorname{sim} 1$ and $0.23 \mathrm{~g}^{-\mathrm{L}^{-1}} \mathrm{day}^{-1}$ for the wild-type. Additionally, the $\mathrm{s} / \mathrm{m} 1$ reached the maximum TAG yield on light within 7.7 days, while the wild-type required 9 days (Remmers et al., 2017).

An attempt to overcome the loss of photosynthetic efficiency observed under nitrogen starvation involved the use of continuous nitrogen limitation processes for lipid production (Pruvost et al., 2009; Klok et al., 2013; de Winter et al., 2014; Remmers et al., 2017), where growth and TAG production can occur simultaneously (Klok et al., 2013). When studying the diurnal behaviour of $T$. obliquus under continuous nitrogen limitation and light/dark cycles, we found that starch is still used as diurnal energy storage compound (Chapter 5). In general, the wild-type is more efficient than the slm1, as was also observed under nitrogen replete conditions. Under these limiting conditions the better performance of the wild-type becomes more evident during the second half of the light period, where starch accumulation occurs.

Overall, however, T. obliquus wild-type and $\operatorname{s} / m 1$ showed lower TAG yields on light under continuous nitrogen limitation compared to batch nitrogen starvation (Remmers et al., 2017). Under batch nitrogen starvation, starch is still respired, but night respiration was marginal and only occurred during the first 3 days of culture. Eventually, starch was slowly degraded (from $0.40 \mathrm{~g} \cdot \mathrm{gDw}^{-1}$ to approximately $0.20 \mathrm{~g} \cdot \mathrm{gDW}^{-1}$ after 25 days of batch cultivation) while TAG synthesis continued (Remmers et al., 2017). This indicates that under nitrogen starvation, starch acts as an overflow sink for electrons. Once the starch storage capacity gets completely full $\left(0.40 \mathrm{~g} \cdot \mathrm{gDW}^{-1}\right)$, the energy is redirected to TAG synthesis. Starch thus no longer 
functions as a diurnal energy storage compound as the buffer is filled up and starch is hardly used anymore during the night. This hypothesis is supported by the loss in the benefit of LD cycles observed under nitrogen replete conditions, as the same maximum TAG yield on light was found under LD cycles $\left(0.16 \mathrm{gTAG}_{\mathrm{T}} \mathrm{mol}_{\mathrm{ph}}{ }^{-1}\right.$ for the wild-type and $0.20 \mathrm{~g}_{\mathrm{TAG}} \cdot \mathrm{mol}_{\mathrm{ph}}{ }^{-1}$ for the $\mathrm{s} / \mathrm{m} 1$ ) (Remmers et al., 2017) and continuous light $\left(0.14 \mathrm{~g}_{\mathrm{DW}} \cdot \mathrm{mol}_{\mathrm{ph}}{ }^{-1}\right.$ for the wild-type and $0.22 \mathrm{gDw}_{\mathrm{DW}} \cdot \mathrm{mol}_{\mathrm{ph}}{ }^{-1}$ for the $\left.\mathrm{s} / \mathrm{m} 1\right)$ (Breuer et al., 2014). Furthermore, under prolonged nitrogen starvation the photosynthetic capacity to convert photons into biomass was the same for both strains (Breuer et al., 2014; Remmers et al., 2017). Therefore, the capacity to accumulate starch is not relevant for the photosynthetic efficiency during the TAG production phase using a batch nitrogen starvation approach.

For the production of TAG, a nitrogen replete biomass growth phase is required to generate sufficient biomass to later produce TAG under nitrogen deplete conditions. The light used in this growth phase should be included in the overall yield of TAG on light. When looking at the growth phase (nitrogen replete growth conditions), the photosynthetic capacity to convert photons into biomass is reduced in the s/m1 mutant (Chapter 4) under continuous turbidostat cultivations. The decrease was between 13 to $31 \%$ compared to the performance of the wild-type. Photosynthetic efficiency is one of the main parameters influencing biomass production costs. Ruiz et al. (2016) found a potential $47 \%$ reduction in cost by doubling the photosynthetic efficiency from 2.7 to $6 \%$. The reduction in photosynthetic efficiency observed in the $\operatorname{sim} 1$ is thus undesirable.

When choosing a batch strategy for the growth phase instead, the outcome might be different than the one obtained under continuous cultivation. When comparing the performance of $T$. obliquus wild-type and $s / m 1$ in shake flasks and continuous light, de Jaeger et al. (2014) found a similar pattern in growth, with a slightly lower biomass concentration by the $\operatorname{s} / m 1$ after 12 days of cultivation. However, a detailed evaluation of the performance (e.g. growth and energy efficiency) under LD cycles and batch conditions still needs to be done.

In summary, a possible explanation for the results under different environmental conditions is that during the light period the capacity to turn light into functional biomass is limiting the yield of biomass on light. The metabolic pathway to make starch 
takes relatively a low amount of enzyme, so a higher yield of biomass on light can be reached by trading in a little bit of the capacity to make functional biomass during the light period for capacity to make starch. In this way, the cells can harvest more light during the day, while forming a little bit less functional biomass during the day. However, this is compensated by using the starch to make functional biomass during the night. In other words, the reduction in machinery to make functional biomass is resulting in less functional biomass during the day. However due to the storage of starch, this machinery can now also be used during the night, resulting overall in an increased biomass yield on light. This mechanism becomes extra advantageous if the algae schedule processes at night that limit the harvest of light during the day (such as under diurnal LD cycles). For example, if during cell division the efficiency to harvest light is decreased, it would be most favourable to schedule this process in the night. In addition, the availability of starch at the beginning of the light period may allow cells to build up the photosynthetic machinery quickly, which may also result in more efficient use of the light.

Under continuous light and nitrogen replete conditions, the biomass yield on light would then be lower because light is supplied during a period where algae cannot use light efficiently, which normally falls during the dark period. The wild-type is still more efficient than the $\operatorname{s} / m 1$ under continuous light because starch synthesis still occurs in the unsynchronized cells, providing a small benefit. This then also explains why for the slm1 mutant there is no difference between LD and continuous light, because starch synthesis is blocked.

However, under LD cycles with longer nights the yield of biomass on light decreases again and the advantage compared to continuous light is not present anymore. This could be linked to higher maintenance requirements, related to longer nights, resulting in loss of energy. However, the yield of biomass on light is still higher for the wild-type than for the mutant, showing that the wild-type is still able to harvest more light energy.

Under nitrogen starvation, the starch capacity is quickly filled-up in the wild-type, blocking additional harvesting of light. Besides, the use of starch during the night becomes minimal as cell division and nitrogen fixation to proteins is no longer active. Therefore, also the difference between LD cycles and continuous light disappears. The 
situation under nitrogen limitation is in-between the nitrogen replete and deplete conditions, where starch still provides a small benefit as it can be used during the dark period, but to a lesser extent.

\subsection{Future directions for better understanding starch and TAG metabolism}

Metabolic models constitute a powerful tool for finding targets for metabolic engineering or elucidating regulatory mechanisms in a metabolic network (Feist and Palsson, 2010) as they allow an overview of all the metabolic reactions. In addition, they summarize what we currently know about metabolism and allow us to see what information is missing in terms of reactions (and thus genes), and localisation. More specifically for algae, models can provide a better insight into metabolism under specific conditions for further improvements on TAG yield on light. Metabolic models for different microalgae have been developed with different number of reactions, metabolites, and degrees of compartmentalisation (Chapter 6) (Yang et al., 2000; Boyle and Morgan, 2009; Manichaikul et al., 2009; Chang et al., 2011; Cogne et al., 2011; Dal'Molin et al., 2011; Kliphuis et al., 2012; Imam et al., 2015).

However the development of metabolic models for algae is currently hindered by problems with annotation and localisation. Annotation of microalgal genomes is usually done by inferred homology with Arabidopsis thaliana (Reijnders et al., 2014) using predicting tools such as BLAST (basic local alignment search tool) (Altschul et al., 1997). Problems with annotation for Tetradesmus obliquus were found when developing the model in Chapter 6, where some lipid desaturase were not present in the genome. However, T. obliquus is able to synthesise these fatty acids (Sharma et al., 2015). In the fatty acid analysis of the samples under nitrogen limitation (Chapter 5), the presence of one of these fatty acids was also found.

When looking into the primary metabolism of eukaryotic microalgae, problems with annotation are less present, while localisation of enzymes is a bigger problem. Localisation has consequences on, for example, redox balancing, as the redox cofactors cannot freely move between compartments, and the ATP consumption, as some transporters use ATP. Additionally, understanding the localisation of processes in the 
cell can be relevant for strain improvement through metabolic engineering. Studies in other eukaryotic microorganisms have shown that targeting a pathway that is compartment specific offers the advantages of higher availability of intermediates, removing the need to transport intermediates and reducing the loss of intermediates to competing pathways (Avalos et al., 2013). For example, in Saccharomyses cerevisiae the over expression of mitochondrial enzymes involved in the production of isobutanol were $260 \%$ higher, compared to only $10 \%$ when overexpressing the enzymes in the cytoplasm (Avalos et al., 2013). In the case of microalgae, the chloroplast would be the obvious organelle to target as the carbon fixated by photosynthesis could be diverted into novel pathways (such as TAG production) (Purton et al., 2013). The algal chloroplast of the model microalgae Chlamydomonas reinhardtii has been successfully transformed (Boynton et al., 1988; Takahashi et al., 1991).

To better understand metabolism inside the chloroplast, this organelle needs to be isolated. The steps and critical factors to isolate the chloroplast are presented in Box 7.2. Once the organelle has been isolated, strategies such as proteomic analyses can be used to study the presence of specific proteins. The chloroplast is a relevant organelle to study the role of starch, as this is the place where starch synthesis and accumulation in granules occurs. Starch metabolism and the associated sugar metabolism is compartmentalized between the chloroplast, cytosol and the mitochondrion. Biosynthesis of starch occurs in the chloroplast, while the sugars are transported through the cytosol to the mitochondrion to be respired. However, gaps still remain. For example, the location where starch is converted into TAG, a process that has been suggested to occur under batch nitrogen starvation conditions (Breuer et al., 2014). 


\section{Box 7.2 Chloroplast isolation}

The chloroplast is the location of many metabolic processes, including photosynthesis, amino-acid biosynthesis, and chlorophyll biosynthesis. Many of these reactions occur in the stroma of the chloroplast and isolation of intact chloroplast allows the study of these processes. However, obtaining intact chloroplast is difficult (Mason et al., 2006). Here we present the critical steps found in literature and the results of our attempts to isolate the chloroplast of Tetradesmus obliquus.

\section{Cell disruption}

The first step is disruption of the cells. Cell disruption should be performed under mild conditions in order to keep the native structure of the individual cell components ('t Lam et al., 2017). The disruption method is strain-dependent, as the composition of the cell wall, and therefore the strength, varies depending on the strain. This method should be strong enough to break the cell wall while preserving the chloroplast intactness. In the case of the model microalgae Chlamydomonas reinhardtii, the first report on chloroplast isolation was in 1983, where the authors disrupted the cell wall by using auto-lysine digestion (Klein et al., 1983). For Chlorella protothecoides, sonication was successful to break the cell wall (Angelova et al., 2013). Subsequent methods reported the isolation of intact chloroplasts from cellwall-deficient mutants of C. reinhardtii (Mendiola-Morgenthaler et al., 1985; Mason et al., 1991).

For Tetradesmus obliquus, different methods to break the cell wall were tested, where usage of beads of different sizes $(<106 \mu \mathrm{m}, 150-212 \mu \mathrm{m}, 212-300 \mu \mathrm{m}$, and 425-600 $\mu \mathrm{m}$ ) showed to successfully destroy the cells (Figure 7.4). Since a mechanical breakage method was shown to be successful, the usage of the bead milling for large volumes is a good alternative (Postma et al., 2015). However, an optimization in biomass concentration, bead size, and milling time is needed in order to use this method.

When using a cell-wall-deficient strain, a mild method can be used to release the cell content, such as the one presented by Mason et al. (1991). Here, the cell-wall-less 
strain $c c-400$ of $C$. reinhardtii was successfully broken by passing them through a stainless steel needle, generating a pressure of approximately 80 p.s.i. (5.51 Bar).

To verify the efficiency of the cell wall disruption technique, methods such as staining with calcofluor white, a dye that binds to chitin and cellulose in cell walls, can be used (Figure 7.5).

\section{Chloroplast isolation}

Chloroplast enrichment or isolation from the cell debris mix is usually achieved using a density gradient centrifugation. Mason et al. (2006) utilized a percoll gradient, separating the cell mix into chloroplasts, thylakoids, and whole cells. Angelova et al. (2013) used a sucrose gradient, resulting in a 2.36-fold enrichment of the chloroplast fraction of $C$. protothecoides that could be used for genome sequencing.
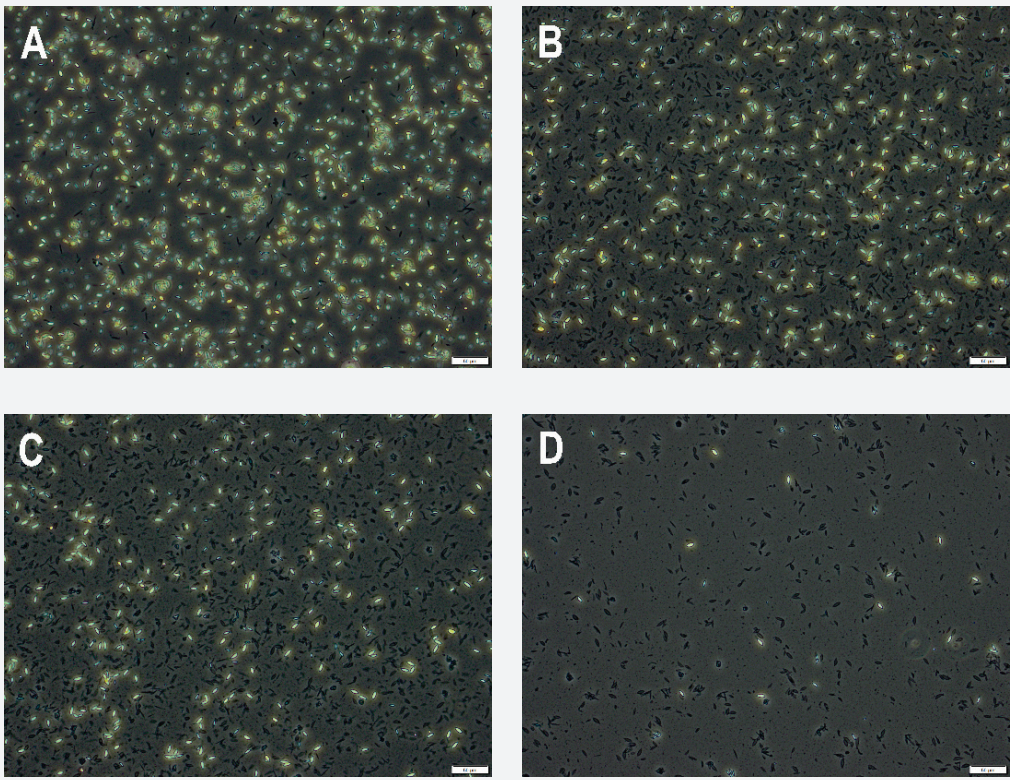

Figure 7.4. Microscope photographs of Tetradesmus obliquus cells treated with the bead beater for $10 \mathrm{~min}$ using different bead sizes: A) $\langle 106 \mu \mathrm{m}$; B) 150 $212 \mu \mathrm{m}$; C) 212-300 $\mu \mathrm{m}$; and D) 425-600 $\mu \mathrm{m}$. Scale bars represent $50 \mu \mathrm{m}$.

To test the purity of the isolated chloroplasts, the presence of other components has to be assessed. One method is by means of enzyme activity essays for enzymes 
present in the other compartments (Mason et al., 1991), such as the cytosolic phospho(enol)pyruvate (PEP) carboxylase and the mitochondrial Cytochrome $c$ Oxidase. Another method suitable for compartments containing DNA is the Polymerase Chain Reaction (PCR), designing marker sequences with selective primers for the chloroplast genome and for the mitochondrial genome.

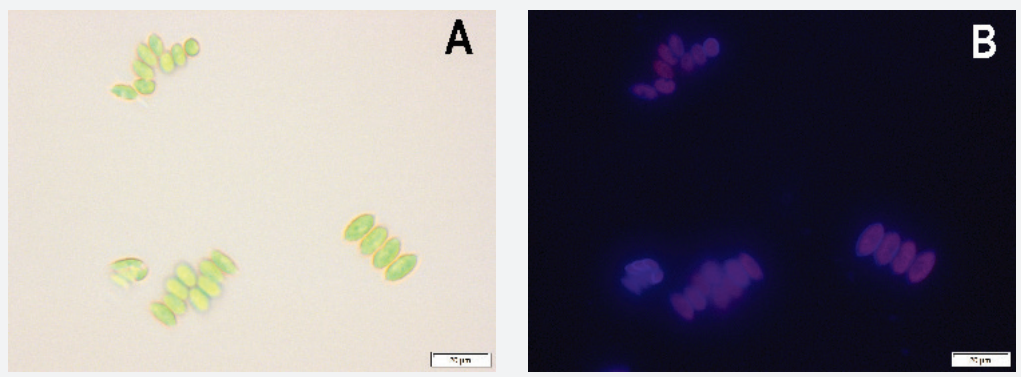

Figure 7.5. Microscope photographs of calcofluor white stained cells of Tetradesmus obliquus observed in bright field (A) and UV excitation (B). All cells contain the cell wall.

\section{Verification of intactness and functionality}

Finally, if necessary, the intactness and functionality of the chloroplasts can be assessed. For the intactness, Schulz et al. (2004) reported a specifically staining method for intact chloroplasts of the model plant Arabidopsis thaliana, using the fluorescent dye carboxyfluorescein diacetate. Another method is by observation using electron microscopy (Mason et al., 1991).

For the functionality, testing the enzyme activity of Rubisco (Ribulose-1,5bisphosphate carboxylase/oxygenase) would be the common approach, as this enzyme is only present in the chloroplast. Another approach would be to test for the $\mathrm{O}_{2}$ evolution and $\mathrm{CO}_{2}$ fixation (Mendiola-Morgenthaler et al., 1985).

Altogether, a method for chloroplast isolation must be tailored to the organism of interest. This includes the composition of the buffers (for breaking and isolating), density gradient used, and the disruption method. 


\subsection{Conclusions}

The role of starch in microalgae depends on the environmental conditions. During the growth phase under nitrogen replete conditions, starch serves as a short term diurnal compound to store energy during the light period that is used during the dark period to support metabolism (e.g. cell division, and nitrogen fixation). The synchronization and usage of starch allow a higher photosynthetic efficiency in the microalga Tetradesmus obliquus. Additionally, starch is consumed during the beginning of the light period, where photosynthesis is also active, probably providing additional energy and carbon to produce pigments in order to be able to rapidly harvest light energy. For replete conditions, the advantage of being able to accumulate starch could be explained by a redistribution of enzyme capacity from functional biomass synthesis to starch synthesis, allowing cells to harvest more energy during the day and allowing them to use their capacity to make functional biomass during the night.

For the lipid production phase, two strategies have been tested: continuous nitrogen limitation and batch nitrogen starvation. Under nitrogen limitation, starch still functions as a diurnal energy storage compound, as oscillations were observed during the diurnal cycle. This allowed a higher photosynthetic efficiency in T. obliquus wildtype compared to the starchless mutant $s / m 1$. Under batch nitrogen starvation, which is the preferred strategy to produce TAG, both starch and TAG are synthesised in T. obliquus wild-type, with starch as the preferred storage compound (up to a content of $\left.0.40 \mathrm{~g} \cdot \mathrm{gDw}^{-1}\right)$, followed by TAG accumulation. However, the benefit observed under light/dark cycles is absent as the capacity to store starch is fully used. During batch nitrogen starvation, the difference in photosynthetic efficiency of both the wild-type and the $\operatorname{sim} 1$ is negligible. Thus under this condition, starch serves as a sink for electrons and as a long term storage compound of carbon and energy that can be used for recovery once nitrogen is again available. 


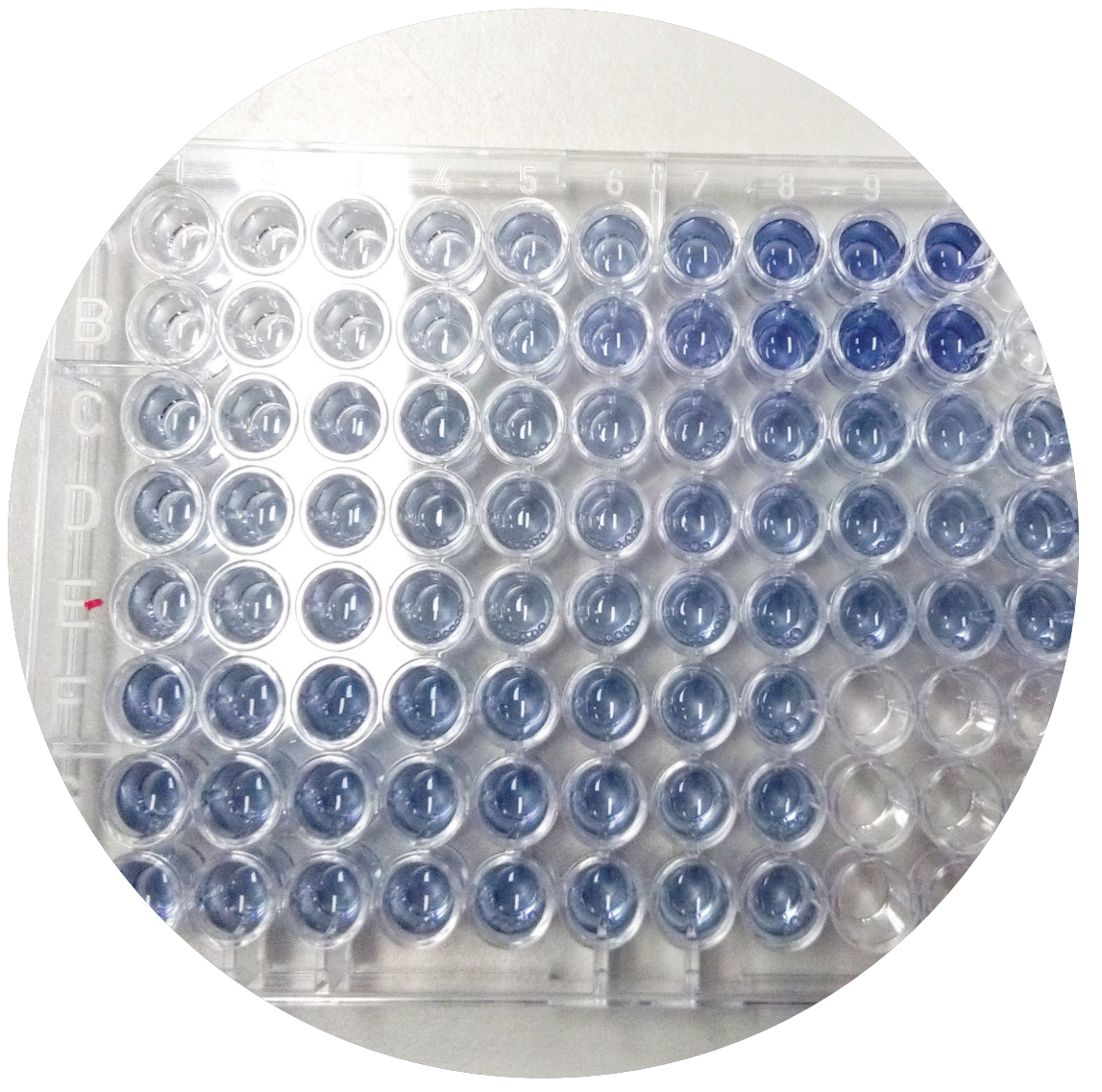


References 
Äijö T, Butty V, Chen Z, Salo V, Tripathi S, Burge CB, Lahesmaa R, Lähdesmäki H (2014) Methods for time series analysis of RNA-seq data with application to human Th17 cell differentiation. Bioinformatics 30: i113-i120

Alberts B, Johnson A, Lewis J, Raff M, Roberts K, Walter P (2002) Molecular Biology of the Cell, 4th edition.

Altschul SF, Madden TL, Schäffer AA, Zhang J, Zhang Z, Miller W, Lipman DJ (1997) Gapped BLAST and PSI-BLAST: a new generation of protein database search programs. Nucleic Acids Res 25: 33893402

Angelova A, Park S-H, Kyndt J, Fitzsimmons K, Brown JK (2013) Sonication-based isolation and enrichment of Chlorella protothecoides chloroplasts for Illumina genome sequencing. J Appl Phycol 26: 209-218

Atteia A, Adrait A, Brugière S, Tardif M, van Lis R, Deusch O, Dagan T, Kuhn L, Gontero B, Martin W, et al (2009) A Proteomic Survey of Chlamydomonas reinhardtii Mitochondria Sheds New Light on the Metabolic Plasticity of the Organelle and on the Nature of the $\alpha$-Proteobacterial Mitochondrial Ancestor. Mol Biol Evol 26: 1533-1548

Avalos JL, Fink GR, Stephanopoulos G (2013) Compartmentalization of metabolic pathways in yeast mitochondria improves the production of branched-chain alcohols. Nat Biotechnol 31: 335341

Baker NR, Harbinson J, Kramer DM (2007) Determining the limitations and regulation of photosynthetic energy transduction in leaves. Plant Cell Environ 30: 1107-1125

Ball SG, Dirick L, Decq A, Martiat J-C, Matagne R (1990) Physiology of starch storage in the monocellular alga Chlamydomonas reinhardtii. Plant Sci 66: 1-9

Ballin G, Doucha J, Zachleder V, Šetlík I (1988) Macromolecular syntheses and the course of cell cycle events in the chlorococcal alga Scenedesmus quadricauda under nutrient starvation: Effect of nitrogen starvation. Biol Plant 30: 81-91

Banerjee A, Maiti SK, Guria C, Banerjee C (2017) Metabolic pathways for lipid synthesis under nitrogen stress in Chlamydomonas and Nannochloropsis. Biotechnol Lett 39: 1-11

Becker SA, Feist AM, Mo ML, Hannum G, Palsson B $\varnothing$, Herrgard MJ (2007) Quantitative prediction of cellular metabolism with constraint-based models: the COBRA Toolbox. Nat Protoc 2: 727-738

Benvenuti G, Bosma R, Klok AJ, Ji F, Lamers PP, Barbosa MJ, Wijffels RH (2015) Microalgal triacylglycerides production in outdoor batch-operated tubular PBRs. Biotechnol Biofuels 8: 100

Benvenuti G, Lamers PP, Breuer G, Bosma R, Cerar A, Wijffels RH, Barbosa MJ (2016) Microalgal TAG production strategies: why batch beats repeated-batch. Biotechnol Biofuels 9: 64

Berg JM, Tymoczko JL, Stryer L, Berg JM, Tymoczko JL, Stryer L (2002) Biochemistry, 5th ed. W H Freeman

Bishop NI, Senger H (1971) Preparation and photosynthetic properties of synchronous cultures of Scenedesmus. In B-M in Enzymology, ed,Academic Press, pp 53-66

Bišová K, Zachleder V (2014) Cell-cycle regulation in green algae dividing by multiple fission. J Exp Bot ert466

Blanken W, Cuaresma M, Wijffels RH, Janssen M (2013) Cultivation of microalgae on artificial light comes at a cost. Algal Res 2: 333-340

Blanken W, Magalhães A, Sebestyén P, Rinzema A, Wijffels RH, Janssen M (2017) Microalgal biofilm growth under day-night cycles. Algal Res 21: 16-26 
Bongers L (1958) Changes in photosynthetic activity during algal growth and multiplication. Meded Landbouwhogesch Wagening 58: 1-10

Borchardt JA, Azad HS (1968) Biological Extraction of Nutrients. J Water Pollut Control Fed 40: 17391754

Boyle NR, Morgan JA (2009) Flux balance analysis of primary metabolism in Chlamydomonas reinhardtii. BMC Syst Biol 3: 4

Boynton JE, Gillham NW, Harris EH, Hosler JP, Johnson AM, Jones AR, Randolph-Anderson BL, Robertson D, Klein TM, Shark KB (1988) Chloroplast transformation in Chlamydomonas with high velocity microprojectiles. Science 240: 1534-1538

Brányiková I, Maršálková B, Doucha J, Brányik T, Bišová K, Zachleder V, Vítová M (2011) Microalgaenovel highly efficient starch producers. Biotechnol Bioeng 108: 766-776

Breuer G, Evers WAC, de Vree JH, Kleinegris DMM, Martens DE, Wijffels RH, Lamers PP (2013a) Analysis of Fatty Acid Content and Composition in Microalgae. J Vis Exp. doi: 10.3791/50628

Breuer G, de Jaeger L, Artus VG, Martens DE, Springer J, Draaisma RB, Eggink G, Wijffels RH, Lamers PP (2014) Superior triacylglycerol (TAG) accumulation in starchless mutants of Scenedesmus obliquus: (II) evaluation of TAG yield and productivity in controlled photobioreactors. Biotechnol Biofuels 7: 70

Breuer G, Lamers PP, Janssen M, Wijffels RH, Martens DE (2015a) Opportunities to improve the areal oil productivity of microalgae. Bioresour Technol 186: 294-302

Breuer G, Lamers PP, Martens DE, Draaisma RB, Wijffels RH (2012) The impact of nitrogen starvation on the dynamics of triacylglycerol accumulation in nine microalgae strains. Bioresour Technol 124: $217-26$

Breuer G, Lamers PP, Martens DE, Draaisma RB, Wijffels RH (2013b) Effect of light intensity, pH, and temperature on triacylglycerol (TAG) accumulation induced by nitrogen starvation in Scenedesmus obliquus. Bioresour Technol 143: 1-9

Breuer G, Martens DE, Draaisma RB, Wijffels RH, Lamers PP (2015b) Photosynthetic efficiency and carbon partitioning in nitrogen-starved Scenedesmus obliquus. Algal Res 9: 254-262

Busi MV, Barchiesi J, Martín M, Gomez-Casati DF (2014) Starch metabolism in green algae. Starch Stärke 66: 28-40

Carlson M, Falcon S, Pages H, Li N (2007) A set of annotation maps describing the entire Gene Ontology. ftp://ctan.uib.no/pub/bioconductor/2.7/data/annotation/html/GO.db.html

Carreres BM, Jaeger L de, Springer J, Barbosa MJ, Breuer G, End EJ van den, Kleinegris DMM, Schäffers I, Wolbert EJH, Zhang H, et al (2017) Draft Genome Sequence of the Oleaginous Green Alga Tetradesmus obliquus UTEX 393. Genome Announc 5: e01449-16

Caspar T, Huber SC, Somerville C (1985) Alterations in Growth, Photosynthesis, and Respiration in a Starchless Mutant of Arabidopsis thaliana (L.) Deficient in Chloroplast Phosphoglucomutase Activity. Plant Physiol 79: 11-17

Causton HC, Feeney KA, Ziegler CA, O'Neill JS (2015) Metabolic Cycles in Yeast Share Features Conserved among Circadian Rhythms. Curr Biol 25: 1056-1062

Chang RL, Ghamsari L, Manichaikul A, Hom EFY, Balaji S, Fu W, Shen Y, Hao T, Palsson B $\emptyset$, SalehiAshtiani K, et al (2011) Metabolic network reconstruction of Chlamydomonas offers insight into light-driven algal metabolism. Mol Syst Biol 7: n/a-n/a 
Chauton MS, Winge P, Brembu T, Vadstein O, Bones AM (2013) Gene Regulation of Carbon Fixation, Storage, and Utilization in the Diatom Phaeodactylum tricornutum Acclimated to Light/Dark Cycles. Plant Physiol 161: 1034-1048

Chen F, Johns MR (1996) Relationship between substrate inhibition and maintenance energy of Chlamydomonas reinhardtii in heterotrophic culture. J Appl Phycol 8: 15-19

Chisti Y (2007) Biodiesel from microalgae. Biotechnol Adv 25: 294-306

Chisti Y (2013) Constraints to commercialization of algal fuels. J Biotechnol 167: 201-214

Cogne G, Rügen M, Bockmayr A, Titica M, Dussap C-G, Cornet J-F, Legrand J (2011) A model-based method for investigating bioenergetic processes in autotrophically growing eukaryotic microalgae: Application to the green algae Chlamydomonas reinhardtii. Biotechnol Prog 27: 631-640

Dal'Molin CG de O, Quek L-E, Palfreyman RW, Nielsen LK (2011) AlgaGEM - a genome-scale metabolic reconstruction of algae based on the Chlamydomonas reinhardtii genome. BMC Genomics 12: S5

Dam JCJ van, Koehorst JJJ, Vik JO, Schaap PJ, Suarez-Diez M (2017) Interoperable genome annotation with GBOL, an extendable infrastructure for functional data mining. bioRxiv 184747

Dobin A, Davis CA, Schlesinger F, Drenkow J, Zaleski C, Jha S, Batut P, Chaisson M, Gingeras TR (2013) STAR: ultrafast universal RNA-seq aligner. Bioinformatics 29: 15-21

DuBois M, Gilles KA, Hamilton JK, Rebers PA, Smith F (1956) Colorimetric Method for Determination of Sugars and Related Substances. Anal Chem 28: 350-356

Edmundson SJ, Huesemann MH (2015) The dark side of algae cultivation: Characterizing night biomass loss in three photosynthetic algae, Chlorella sorokiniana, Nannochloropsis salina and Picochlorum sp. Algal Res 12: 470-476

Fábregas J, Maseda A, Domínguez A, Ferreira M, Otero A (2002) Changes in the cell composition of the marine microalga, Nannochloropsis gaditana, during a light:dark cycle. Biotechnol Lett 24: 1699-1703

Falda M, Toppo S, Pescarolo A, Lavezzo E, Di Camillo B, Facchinetti A, Cilia E, Velasco R, Fontana P (2012) Argot2: a large scale function prediction tool relying on semantic similarity of weighted Gene Ontology terms. BMC Bioinformatics 13: S14

Fan J, Yan C, Andre C, Shanklin J, Schwender J, Xu C (2012) Oil accumulation is controlled by carbon precursor supply for fatty acid synthesis in Chlamydomonas reinhardtii. Plant Cell Physiol 53: 1380-1390

Farré EM (2012) The regulation of plant growth by the circadian clock. Plant Biol 14: 401-410

Feist AM, Henry CS, Reed JL, Krummenacker M, Joyce AR, Karp PD, Broadbelt L, Hatzimanikatis V, Palsson B $\varnothing$ (2007) A genome-scale metabolic reconstruction for Escherichia coli K-12 MG1655 that accounts for 1260 ORFs and thermodynamic information. Mol Syst Biol 3: 121

Feist AM, Palsson BO (2010) The biomass objective function. Curr Opin Microbiol 13: 344-349

Fernandes B, Teixeira J, Dragone G, Vicente AA, Kawano S, Bišová K, Přibyl P, Zachleder V, Vítová M (2013) Relationship between starch and lipid accumulation induced by nutrient depletion and replenishment in the microalga Parachlorella kessleri. Bioresour Technol 144: 268-274

Förster J, Famili I, Fu P, Palsson B $\varnothing$, Nielsen J (2003) Genome-Scale Reconstruction of the Saccharomyces cerevisiae Metabolic Network. Genome Res 13: 244-253

Fujiwara T, Misumi O, Tashiro K, Yoshida Y, Nishida K, Yagisawa F, Imamura S, Yoshida M, Mori T, Tanaka K, et al (2009) Periodic Gene Expression Patterns during the Highly Synchronized Cell 
Nucleus and Organelle Division Cycles in the Unicellular Red Alga Cyanidioschyzon merolae. DNA Res Int J Rapid Publ Rep Genes Genomes 16: 59-72

Garz A, Sandmann M, Rading M, Ramm S, Menzel R, Steup M (2012) Cell-to-Cell Diversity in a Synchronized Chlamydomonas Culture As Revealed by Single-Cell Analyses. Biophys J 103: 1078-1086

Goldbeter A, Gérard C, Gonze D, Leloup J-C, Dupont G (2012) Systems biology of cellular rhythms. FEBS Lett 586: 2955-2965

Graf A, Schlereth A, Stitt M, Smith AM (2010) Circadian control of carbohydrate availability for growth in Arabidopsis plants at night. Proc Natl Acad Sci 107: 9458-9463

Guarnieri MT, Pienkos PT (2015) Algal omics: unlocking bioproduct diversity in algae cell factories. Photosynth Res 123: 255-263

Hennig C (2018) fpc: Flexible Procedures for Clustering. https://CRAN.R-project.org/package=fpc

Herbert, D., Phipps, P. J., Strange, R. E. (1971) Chemical analysis of microbial cells. Methods Microbiol. Academic Press, London and New York, pp 209-344

Ho S-H, Chen C-Y, Chang J-S (2012) Effect of light intensity and nitrogen starvation on CO2 fixation and lipid/carbohydrate production of an indigenous microalga Scenedesmus obliquus CNW-N. Bioresour Technol 113: 244-252

Hoefnagel MH., Atkin OK, Wiskich JT (1998) Interdependence between chloroplasts and mitochondria in the light and the dark. Biochim Biophys Acta BBA - Bioenerg 1366: 235-255

Hoff KJ, Lange S, Lomsadze A, Borodovsky M, Stanke M (2016) BRAKER1: Unsupervised RNA-SeqBased Genome Annotation with GeneMark-ET and AUGUSTUS. Bioinformatics 32: 767-769

Hu Q, Sommerfeld M, Jarvis E, Ghirardi M, Posewitz M, Seibert M, Darzins A (2008) Microalgal triacylglycerols as feedstocks for biofuel production: perspectives and advances. Plant J 54: 621-639

Imam S, Schäuble S, Valenzuela J, López García de Lomana A, Carter W, Price ND, Baliga NS (2015) A refined genome-scale reconstruction of Chlamydomonas metabolism provides a platform for systems-level analyses. Plant J 84: 1239-1256

Jacob-Lopes E, Scoparo CHG, Lacerda LMCF, Franco TT (2009) Effect of light cycles (night/day) on CO2 fixation and biomass production by microalgae in photobioreactors. Chem Eng Process Process Intensif 48: 306-310

de Jaeger L (2015) Strain Improvement of Oleaginous Microalgae. Bioprocess Engineering, Wageningen UR

de Jaeger L, Verbeek RE, Draaisma RB, Martens DE, Springer J, Eggink G, Wijffels RH (2014) Superior triacylglycerol (TAG) accumulation in starchless mutants of Scenedesmus obliquus: (I) mutant generation and characterization. Biotechnol Biofuels 7: 69

Jia J, Han D, Gerken HG, Li Y, Sommerfeld M, Hu Q, Xu J (2015) Molecular mechanisms for photosynthetic carbon partitioning into storage neutral lipids in Nannochloropsis oceanica under nitrogen-depletion conditions. Algal Res 7: 66-77

Johnson X, Alric J (2013) Central Carbon Metabolism and Electron Transport in Chlamydomonas reinhardtii: Metabolic Constraints for Carbon Partitioning between Oil and Starch. Eukaryot Cell 12: 776-793

Johnson X, Alric J (2012) Interaction between Starch Breakdown, Acetate Assimilation, and Photosynthetic Cyclic Electron Flow in Chlamydomonas reinhardtii. 26445-26452 
Jones P, Binns D, Chang H-Y, Fraser M, Li W, McAnulla C, McWilliam H, Maslen J, Mitchell A, Nuka G, et al (2014) InterProScan 5: genome-scale protein function classification. Bioinformatics 30: 1236-1240

Kanehisa M, Goto S (2000) KEGG: kyoto encyclopedia of genes and genomes. Nucleic Acids Res 28: 27-30

Kanehisa M, Sato Y, Kawashima M, Furumichi M, Tanabe M (2016) KEGG as a reference resource for gene and protein annotation. Nucleic Acids Res 44: D457-462

Kayser A, Weber J, Hecht V, Rinas U (2005) Metabolic flux analysis of Escherichia coli in glucose-limited continuous culture. I. Growth-rate-dependent metabolic efficiency at steady state. Microbiology 151: 693-706

Klein U (1987) Intracellular Carbon Partitioning in Chlamydomonas reinhardtii. Plant Physiol 85: 892897

Klein U, Chen C, Gibbs M, Platt-Aloia KA (1983) Cellular Fractionation of Chlamydomonas reinhardii with Emphasis on the Isolation of the Chloroplast. Plant Physiol 72: 481-487

Kliphuis AMJ, Klok AJ, Martens DE, Lamers PP, Janssen M, Wijffels RH (2012) Metabolic modeling of Chlamydomonas reinhardtii: energy requirements for photoautotrophic growth and maintenance. J Appl Phycol 24: 253-266

Klok AJ (2013) Lipid production in microalgae. Bioprocess Engineering, Wageningen UR

Klok AJ, Lamers PP, Martens DE, Draaisma RB, Wijffels RH (2014) Edible oils from microalgae: insights in TAG accumulation. Trends Biotechnol 32: 521-528

Klok AJ, Martens DE, Wijffels RH, Lamers PP (2013) Simultaneous growth and neutral lipid accumulation in microalgae. Bioresour Technol 134: 233-243

Koehorst JJ, Dam V, J JC, Saccenti E, Santos M dos, P VA, Suarez-Diez M, Schaap PJ (2018) SAPP: functional genome annotation and analysis through a semantic framework using FAIR principles. Bioinformatics 34: 1401-1403

Krienitz L, Bock C (2012) Present state of the systematics of planktonic coccoid green algae of inland waters. Hydrobiologia 698: 295-326

Krzemińska I, Pawlik-Skowrońska B, Trzcińska M, Tys J (2014) Influence of photoperiods on the growth rate and biomass productivity of green microalgae. Bioprocess Biosyst Eng 37: 735741

Lacour T, Sciandra A, Talec A, Mayzaud P, Bernard O (2012) Diel Variations of Carbohydrates and Neutral Lipids in Nitrogen-Sufficient and Nitrogen-Starved Cyclostat Cultures of Isochrysis Sp.1. J Phycol 48: 966-975

Laloi M (1999) Plant mitochondrial carriers: an overview. Cell Mol Life Sci CMLS 56: 918-944

't Lam GP, Vermuë MH, Eppink MHM, Wijffels RH, van den Berg C (2017) Multi-Product Microalgae Biorefineries: From Concept Towards Reality. Trends Biotechnol. doi: 10.1016/j.tibtech.2017.10.011

Lancelot C, Mathot S (1985) Biochemical fractionation of primary production by phytoplankton in Belgian coastal waters during short- and long-term incubations with 14C-bicarbonate. Mar Biol 86: $219-226$

León-Saiki GM, Cabrero Martí T, van der Veen D, Wijffels RH, Martens DE (2018) The impact of day length on cell division and efficiency of light use in a starchless mutant of Tetradesmus obliquus. Algal Res 31: 387-394 
León-Saiki GM, Remmers IM, Martens DE, Lamers PP, Wijffels RH, van der Veen D (2017) The role of starch as transient energy buffer in synchronized microalgal growth in Acutodesmus obliquus. Algal Res 25: 160-167

Li Y, Han D, Hu G, Dauvillee D, Sommerfeld M, Ball S, Hu Q (2010a) Chlamydomonas starchless mutant defective in ADP-glucose pyrophosphorylase hyper-accumulates triacylglycerol. Metab Eng 12: 387-391

Li Y, Han D, Hu G, Sommerfeld M, Hu Q (2010b) Inhibition of starch synthesis results in overproduction of lipids in Chlamydomonas reinhardtii. Biotechnol Bioeng 107: 258-268

Li Y, Han D, Sommerfeld M, Hu Q (2011) Photosynthetic carbon partitioning and lipid production in the oleaginous microalga Pseudochlorococcum sp. (Chlorophyceae) under nitrogen-limited conditions. Bioresour Technol 102: 123-129

Lichtenthaler HK (1987) [34] Chlorophylls and carotenoids: Pigments of photosynthetic biomembranes. In B-M in Enzymology, ed,Academic Press, pp 350-382

Lu H, Cao W, Ouyang L, Xia J, Huang M, Chu J, Zhuang Y, Zhang S, Noorman H (2017) Comprehensive reconstruction and in silico analysis of Aspergillus niger genome-scale metabolic network model that accounts for 1210 ORFs. Biotechnol Bioeng 114: 685-695

Lu Y (2005) Daylength and Circadian Effects on Starch Degradation and Maltose Metabolism. Plant Physiol 138: 2280-2291

Ma X, Liu J, Liu B, Chen T, Yang B, Chen F (2016) Physiological and biochemical changes reveal stressassociated photosynthetic carbon partitioning into triacylglycerol in the oleaginous marine alga Nannochloropsis oculata. Algal Res 16: 28-35

Mandal S, Mallick N (2009) Microalga Scenedesmus obliquus as a potential source for biodiesel production. Appl Microbiol Biotechnol 84: 281-291

Manichaikul A, Ghamsari L, Hom EFY, Lin C, Murray RR, Chang RL, Balaji S, Hao T, Shen Y, Chavali AK, et al (2009) Metabolic network analysis integrated with transcript verification for sequenced genomes. Nat Methods 6: 589-592

Mason CB, Bricker TM, Moroney JV (2006) A rapid method for chloroplast isolation from the green alga Chlamydomonas reinhardtii. Nat Protoc 1: 2227-2230

Mason CB, Matthews S, Bricker TM, Moroney JV (1991) Simplified Procedure for the Isolation of Intact Chloroplasts from Chlamydomonas reinhardtii. Plant Physiol 97: 1576-1580

Mata TM, Martins AA, Caetano NS (2010) Microalgae for biodiesel production and other applications: A review. Renew Sustain Energy Rev 14: 217-232

McClung CR (2006) Plant Circadian Rhythms. Plant Cell 18: 792-803

Mendiola-Morgenthaler L, Leu S, Boschetti A (1985) Isolation of biochemically active chloroplasts from chlamydomonas. Plant Sci 38: 33-39

Meng Y, Jiang J, Wang H, Cao X, Xue S, Yang Q, Wang W (2015) The characteristics of TAG and EPA accumulation in Nannochloropsis oceanica IMET1 under different nitrogen supply regimes. Bioresour Technol 179: 483-489

Merchant SS, Kropat J, Liu B, Shaw J, Warakanont J (2012) TAG, You're it! Chlamydomonas as a reference organism for understanding algal triacylglycerol accumulation. Curr Opin Biotechnol 23: 352-363

Merchant SS, Prochnik SE, Vallon O, Harris EH, Karpowicz SJ, Witman GB, Terry A, Salamov A, FritzLaylin LK, Marechal-Drouard L, et al (2007) The Chlamydomonas Genome Reveals the Evolution of Key Animal and Plant Functions. 245-250 
Michelle Wood A, Everroad RC, Wingard LM (2005) Measuring Growth Rates in Microalgal Cultures. In RA Andersen, ed, Algal Cult. Tech. Academic Press, pp 269-285

Michels MHA, Camacho-Rodríguez J, Vermuë MH, Wijffels RH (2014) Effect of cooling in the night on the productivity and biochemical composition of Tetraselmis suecica. Algal Res 6, Part B: 145151

Mittag M (2001) Circadian rhythms in microalgae. In B-IR of Cytology, ed,Academic Press, pp 213-247

de Mooij T, Schediwy K, Wijffels RH, Janssen M (2016) Modeling the competition between antenna size mutant and wild type microalgae in outdoor mass culture. J Biotechnol 240: 1-13

Mulders KJM, Lamers PP, Wijffels RH, Martens DE (2014) Dynamics of biomass composition and growth during recovery of nitrogen-starved Chromochloris zofingiensis. Appl Microbiol Biotechnol 99: 1873-1884

Muthuraj M, Palabhanvi B, Misra S, Kumar V, Sivalingavasu K, Das D (2013) Flux balance analysis of Chlorella sp. FC2 IITG under photoautotrophic and heterotrophic growth conditions. Photosynth Res 118: 167-179

Nguyen N-N, Srihari S, Leong HW, Chong K-F (2015) EnzDP: Improved enzyme annotation for metabolic network reconstruction based on domain composition profiles. J Bioinform Comput Biol 13: 1543003

Nikaido SS, Johnson CH (2000) Daily and Circadian Variation in Survival From Ultraviolet Radiation in Chlamydomonas reinhardtii. Photochem Photobiol 71: 758-765

Niwa Y, Matsuo T, Onai K, Kato D, Tachikawa M, Ishiura M (2013) Phase-resetting mechanism of the circadian clock in Chlamydomonas reinhardtii. Proc Natl Acad Sci 110: 13666-13671

Norsker N-H, Barbosa MJ, Vermuë MH, Wijffels RH (2011) Microalgal production - A close look at the economics. Biotechnol Adv 29: 24-27

Nueda MJ, Tarazona S, Conesa A (2014) Next maSigPro: updating maSigPro bioconductor package for RNA-seq time series. Bioinformatics 30: 2598-2602

Oldenhof H, Zachleder V, Ende H van den (2007) The cell cycle of Chlamydomonas reinhardtii: the role of the commitment point. Folia Microbiol (Praha) 52: 53

Oliveira AP, Nielsen J, Förster J (2005) Modeling Lactococcus lactis using a genome-scale flux model. BMC Microbiol 5: 39

Pirt SJ (1965) The Maintenance Energy of Bacteria in Growing Cultures. Proc R Soc Lond B Biol Sci 163: 224-231

Poiré R, Wiese-Klinkenberg A, Parent B, Mielewczik M, Schurr U, Tardieu F, Walter A (2010) Diel timecourses of leaf growth in monocot and dicot species: endogenous rhythms and temperature effects. J Exp Bot erq049

Poliner E, Panchy N, Newton L, Wu G, Lapinsky A, Bullard B, Zienkiewicz A, Benning C, Shiu S-H, Farré EM (2015) Transcriptional coordination of physiological responses in Nannochloropsis oceanica CCMP1779 under light/dark cycles. Plant J 83: 1097-1113

Postma PR, Miron TL, Olivieri G, Barbosa MJ, Wijffels RH, Eppink MHM (2015) Mild disintegration of the green microalgae Chlorella vulgaris using bead milling. Bioresour Technol 184: 297-304

Powell N, Shilton A, Pratt S, Chisti Y (2011) Luxury uptake of phosphorus by microalgae in full-scale waste stabilisation ponds. Water Sci Technol 63: 704-709

Powell N, Shilton AN, Pratt S, Chisti Y (2008) Factors Influencing Luxury Uptake of Phosphorus by Microalgae in Waste Stabilization Ponds. Environ Sci Technol 42: 5958-5962 
Pruvost J, Van Vooren G, Cogne G, Legrand J (2009) Investigation of biomass and lipids production with Neochloris oleoabundans in photobioreactor. Bioresour Technol 100: 5988-5995

Pulz O, Gross W (2004) Valuable products from biotechnology of microalgae. Appl Microbiol Biotechnol 65: 635-648

Purton S, Szaub JB, Wannathong T, Young R, Economou CK (2013) Genetic engineering of algal chloroplasts: Progress and prospects. Russ J Plant Physiol 60: 491-499

R Core Team (2017) R: A Language and Environment for Statistical Computing. https://www.rproject.org/

Ramazanov A, Ramazanov Z (2006) Isolation and characterization of a starchless mutant of Chlorella pyrenoidosa STL-PI with a high growth rate, and high protein and polyunsaturated fatty acid content. Phycol Res 54: 255-259

Reijnders MJMF, Carreres BM, Schaap PJ (2015) Algal Omics: The Functional Annotation Challenge. Curr Biotechnol 4: 457-463

Reijnders MJMF, van Heck RGA, Lam CMC, Scaife MA, Santos VAPM dos, Smith AG, Schaap PJ (2014) Green genes: bioinformatics and systems-biology innovations drive algal biotechnology. Trends Biotechnol 32: 617-626

Remmers IM, Hidalgo-Ulloa A, Brandt BP, Evers WAC, Wijffels RH, Lamers PP (2017) Continuous versus batch production of lipids in the microalgae Acutodesmus obliquus. Bioresour Technol 244: 1384-1392

Robinson MD, McCarthy DJ, Smyth GK (2010) edgeR: a Bioconductor package for differential expression analysis of digital gene expression data. Bioinformatics 26: 139-140

Robinson MD, Oshlack A (2010) A scaling normalization method for differential expression analysis of RNA-seq data. Genome Biol 11: R25

Rodolfi L, Chini Zittelli G, Bassi N, Padovani G, Biondi N, Bonini G, Tredici MR (2009) Microalgae for oil: strain selection, induction of lipid synthesis and outdoor mass cultivation in a low-cost photobioreactor. Biotechnol Bioeng 102: 100-12

Roenneberg T, Foster RG (1997) Twilight Times: Light and the Circadian System. Photochem Photobiol 66: 549-561

Rosenberg JN, Oyler GA, Wilkinson L, Betenbaugh MJ (2008) A green light for engineered algae: redirecting metabolism to fuel a biotechnology revolution. Curr Opin Biotechnol 19: 430-436

Ruiz J, Olivieri G, Vree J de, Bosma R, Willems P, Hans Reith J, M. Eppink MH, M. Kleinegris DM, H. Wijffels R, J. Barbosa M (2016) Towards industrial products from microalgae. Energy Environ Sci 9: 3036-3043

Salichos L, Rokas A (2010) The diversity and evolution of circadian clock proteins in fungi. Mycologia 102: $269-278$

Schellenberger J, Que R, Fleming RMT, Thiele I, Orth JD, Feist AM, Zielinski DC, Bordbar A, Lewis NE, Rahmanian S, et al (2011) Quantitative prediction of cellular metabolism with constraintbased models: the COBRA Toolbox v2.0. Nat Protoc 6: 1290-1307

Schulz A, Knoetzel J, Scheller HV, Mant A (2004) Uptake of a fluorescent dye as a swift and simple indicator of organelle intactness: import-competent chloroplasts from soil-grown Arabidopsis. J Histochem Cytochem Off J Histochem Soc 52: 701-704

Schulze W, Stitt M, Schulze E-D, Neuhaus HE, Fichtner K (1991) A Quantification of the Significance of Assimilatory Starch for Growth of Arabidopsis thaliana L. Heynh 1. Plant Physiol 95: 890-895 
Senger H (1970) Charakterisierung einer Synchronkultur von Scenedesmus obliquus, ihrer potentiellen Photosyntheseleistung und des Photosynthese-Quotienten während des Entwicklungscyclus. Planta 90: 243-266

Sforza E, Calvaruso C, Meneghesso A, Morosinotto T, Bertucco A (2015a) Effect of specific light supply rate on photosynthetic efficiency of Nannochloropsis salina in a continuous flat plate photobioreactor. Appl Microbiol Biotechnol 99: 8309-8318

Sforza E, Urbani S, Bertucco A (2015b) Evaluation of maintenance energy requirements in the cultivation of Scenedesmus obliquus: effect of light intensity and regime. J Appl Phycol 27: 1453-1462

Sharma DK, Gautam K, Jueppner J, Giavalisco P, Rihko-Struckmann L, Pareek A, Sundmacher K (2015) UPLC-MS analysis of Chlamydomonas reinhardtii and Scenedesmus obliquus lipid extracts and their possible metabolic roles. J Appl Phycol 27: 1149-1159

Shearman LP, Sriram S, Weaver DR, Maywood ES, Chaves I, Zheng B, Kume K, Lee CC, Der GTJ van, Horst, et al (2000) Interacting Molecular Loops in the Mammalian Circadian Clock. Science 288: 1013-1019

Siaut M, Heijde M, Mangogna M, Montsant A, Coesel S, Allen A, Manfredonia A, Falciatore A, Bowler C (2007) Molecular toolbox for studying diatom biology in Phaeodactylum tricornutum. Gene 406: $23-35$

Simionato D, Block MA, Rocca NL, Jouhet J, Maréchal E, Finazzi G, Morosinotto T (2013) The Response of Nannochloropsis gaditana to Nitrogen Starvation Includes De Novo Biosynthesis of Triacylglycerols, a Decrease of Chloroplast Galactolipids, and Reorganization of the Photosynthetic Apparatus. Eukaryot Cell 12: 665-676

Sirikhachornkit A, Vuttipongchaikij S, Suttangkakul A, Yokthongwattana K, Juntawong $P$, Pokethitiyook P, Kangvansaichol K, Meetam M (2016) Increasing the Triacylglycerol Content in Dunaliella tertiolecta through Isolation of Starch-Deficient Mutants. J Microbiol Biotechnol 26: 854-866

Smallbone K, Simeonidis E (2009) Flux balance analysis: A geometric perspective. J Theor Biol 258: 311-315

Smith AM, Stitt M (2007) Coordination of carbon supply and plant growth. Plant Cell Environ 30: 11261149

Smith SM (2004) Diurnal Changes in the Transcriptome Encoding Enzymes of Starch Metabolism Provide Evidence for Both Transcriptional and Posttranscriptional Regulation of Starch Metabolism in Arabidopsis Leaves. Plant Physiol 136: 2687-2699

Spolaore P, Joannis-Cassan C, Duran E, Isambert A (2006) Commercial applications of microalgae. J Biosci Bioeng 101: 87-96

Stephanopoulos G, Aristidou AA, Nielsen J (1998) Metabolic Engineering: Principles and Methodologies. Academic Press

Stern D (2009) The Chlamydomonas Sourcebook: Organellar and Metabolic Processes, 2nd ed. Academic Press

Stitt M, Zeeman SC (2012) Starch turnover: pathways, regulation and role in growth. Curr Opin Plant Biol 15: 282-292

Sulpice R, Pyl E-T, Ishihara H, Trenkamp S, Steinfath M, Witucka-Wall H, Gibon Y, Usadel B, Poree F, Piques MC, et al (2009) Starch as a major integrator in the regulation of plant growth. Proc Natl Acad Sci 106: 10348-10353 
Suzuki L, Johnson CH (2001) Algae Know the Time of Day: Circadian and Photoperiodic Programs. J Phycol 37: 933-942

Suzuki S, Ishida K-I, Hirakawa Y (2016) Diurnal Transcriptional Regulation of Endosymbiotically Derived Genes in the Chlorarachniophyte Bigelowiella natans. Genome Biol Evol 8: 2672-2682

Takahashi Y, Goldschmidt-Clermont M, Soen SY, Franzén LG, Rochaix JD (1991) Directed chloroplast transformation in Chlamydomonas reinhardtii: insertional inactivation of the psaC gene encoding the iron sulfur protein destabilizes photosystem I. EMBO J 10: 2033-2040

Thor S, Peterson JR, Luthey-Schulten Z (2017) Genome-Scale Metabolic Modeling of Archaea Lends Insight into Diversity of Metabolic Function. Archaea. doi: 10.1155/2017/9763848

Trapnell C, Williams BA, Pertea G, Mortazavi A, Kwan G, Baren MJ van, Salzberg SL, Wold BJ, Pachter L (2010) Transcript assembly and quantification by RNA-Seq reveals unannotated transcripts and isoform switching during cell differentiation. Nat Biotechnol 28: 511-515

Vitova M, Bisova K, Kawano S, Zachleder V (2015) Accumulation of energy reserves in algae: From cell cycles to biotechnological applications. Biotechnol Adv 33: 1204-1218

Vítová M, Bišová K, Umysová D, Hlavová M, Kawano S, Zachleder V, Čížková M (2011) Chlamydomonas reinhardtii: duration of its cell cycle and phases at growth rates affected by light intensity. Planta 233: 75-86

Vonlanthen S, Dauvillée D, Purton S (2015) Evaluation of novel starch-deficient mutants of Chlorella sorokiniana for hyper-accumulation of lipids. Algal Res 12: 109-118

Wang ZT, Ullrich N, Joo S, Waffenschmidt S, Goodenough U (2009) Algal lipid bodies: stress induction, purification, and biochemical characterization in wild-type and starchless Chlamydomonas reinhardtii. Eukaryot Cell 8: 1856-68

Wanka F, Joppen MMJ, Kuyper CMA (1970) Starch-degrading enzymes in synchronous cultures of Chlorella. Z. Pflanzenphysiol.

Webb AAR, Satake A (2015) Understanding Circadian Regulation of Carbohydrate Metabolism in Arabidopsis Using Mathematical Models. Plant Cell Physiol 56: 586-593

Weise SE, Wijk KJ van, Sharkey TD (2011) The role of transitory starch in C3, CAM, and C4 metabolism and opportunities for engineering leaf starch accumulation. J Exp Bot err035

Wijffels RH, Barbosa MJ (2010) An Outlook on Microalgal Biofuels. Science 329: 796-799

Wijffels RH, Barbosa MJ, Eppink MHM (2010) Microalgae for the production of bulk chemicals and biofuels. Biofuels Bioprod Biorefining 4: 287-295

Wijffels RH, Kruse O, Hellingwerf KJ (2013) Potential of industrial biotechnology with cyanobacteria and eukaryotic microalgae. Curr Opin Biotechnol 24: 405-413

Wilkinson MD, Dumontier M, Aalbersberg ljJ, Appleton G, Axton M, Baak A, Blomberg N, Boiten JW, Santos LB da S, Bourne PE, et al (2016) The FAIR Guiding Principles for scientific data management and stewardship. Sci Data. doi: 10.1038/sdata.2016.18

Willamme R, Alsafra Z, Arumugam R, Eppe G, Remacle F, Levine RD, Remacle C (2015) Metabolomic analysis of the green microalga Chlamydomonas reinhardtii cultivated under day/night conditions. J Biotechnol 215: 20-26

de Winter L (2015) Circadian rhythms in microalgae production. Bioprocess Engineering, Wageningen UR

de Winter L, Cabanelas ITD, Martens DE, Wijffels RH, Barbosa MJ (2017a) The influence of day/night cycles on biomass yield and composition of Neochloris oleoabundans. Biotechnol Biofuels 10: 104 
de Winter L, Cabanelas ITD, Órfão AN, Vaessen E, Martens DE, Wijffels RH, Barbosa MJ (2017b) The influence of day length on circadian rhythms of Neochloris oleoabundans. Algal Res 22: 31-38

de Winter L, Klok AJ, Cuaresma Franco M, Barbosa MJ, Wijffels RH (2013) The synchronized cell cycle of Neochloris oleoabundans and its influence on biomass composition under constant light conditions. Algal Res 2: 313-320

de Winter L, Schepers LW, Cuaresma M, Barbosa MJ, Martens DE, Wijffels RH (2014) Circadian rhythms in the cell cycle and biomass composition of Neochloris oleoabundans under nitrogen limitation. J Biotechnol 187: 25-33

Work VH, Radakovits R, Jinkerson RE, Meuser JE, Elliott LG, Vinyard DJ, Laurens LML, Dismukes GC, Posewitz MC (2010) Increased lipid accumulation in the Chlamydomonas reinhardtii sta7-10 starchless isoamylase mutant and increased carbohydrate synthesis in complemented strains. Eukaryot Cell 9: 1251-61

Wynne MJ, Hallan JK (2015) Reinstatement of Tetradesmus G. M. Smith (Sphaeropleales, Chlorophyta). Feddes Repert 126: 83-86

Yang C, Hua Q, Shimizu K (2000) Energetics and carbon metabolism during growth of microalgal cells under photoautotrophic, mixotrophic and cyclic light-autotrophic/dark-heterotrophic conditions. Biochem Eng J 6: 87-102

Zijffers J-WF, Schippers KJ, Zheng K, Janssen M, Tramper J, Wijffels RH (2010) Maximum Photosynthetic Yield of Green Microalgae in Photobioreactors. Mar Biotechnol 12: 708-718

Zones JM, Blaby IK, Merchant SS, Umen JG (2015) High-Resolution Profiling of a Synchronized Diurnal Transcriptome from Chlamydomonas reinhardtii Reveals Continuous Cell and Metabolic Differentiation. Plant Cell 27: 2743-2769 


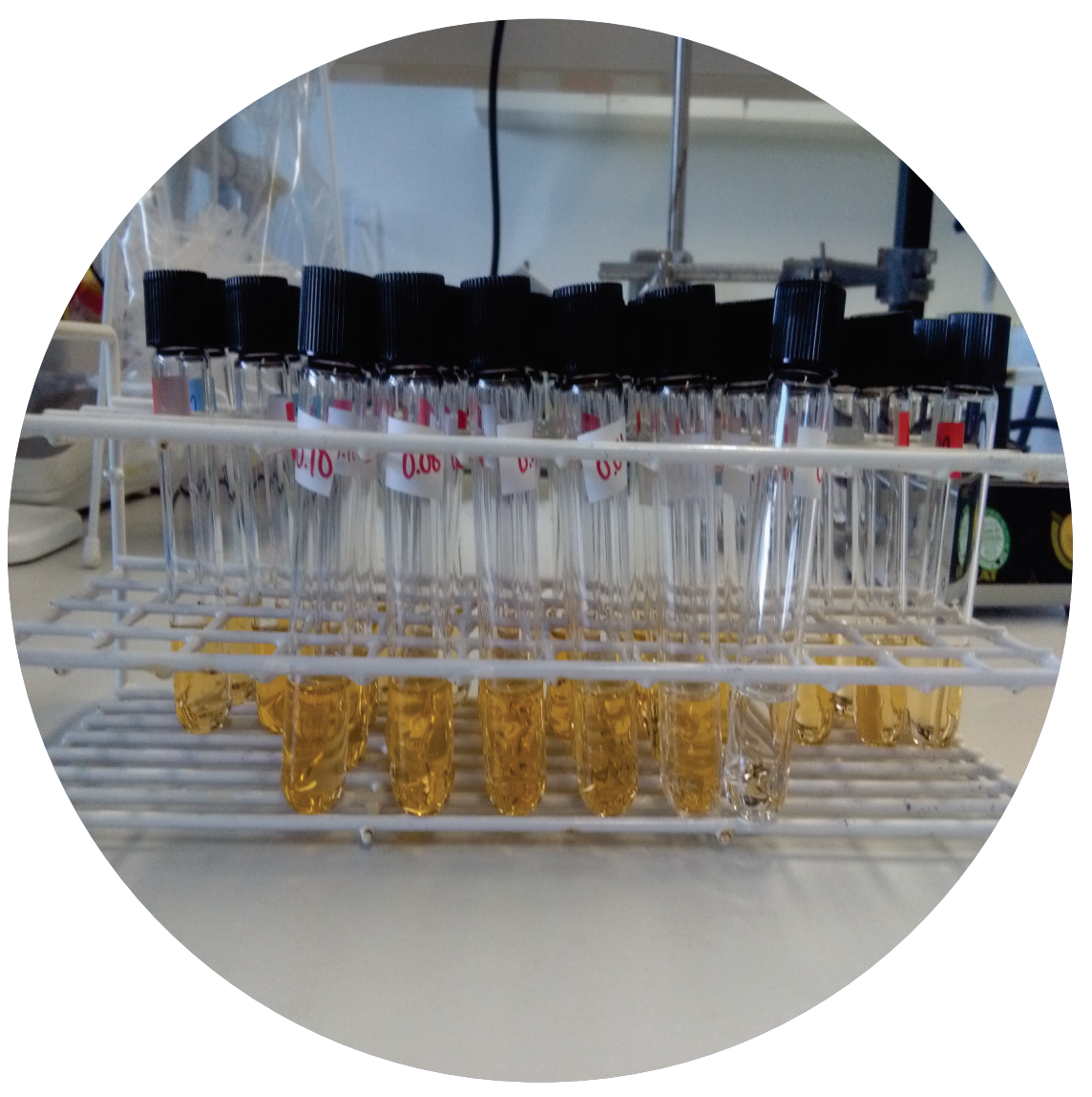




\section{Summary}

Resumen 


\section{Summary}

Microalgae are promising feedstocks for the production of biofuels, foods, feeds, and high value compounds. They are of special interest due to their capacity to produce lipids. Oleaginous microalgae can reach a triacylglycerides (TAG) content of up to $60 \%$ on a dry weight basis under stress conditions, have a higher areal productivity than agricultural crops and do not compete for arable land.

Large scale microalgal production is commonly divided in two phases: the growth phase and the lipid (TAG) production phase. During the growth phase, favourable nitrogen replete conditions are used, while for the TAG production phase mostly nitrogen deplete conditions are used. During the growth phase under nitrogen replete conditions and light/dark (LD) cycles, many photosynthetic microorganisms synchronize their metabolism. During LD cycles, many microalgae store light energy and carbon in the form of starch. This allows microalgae to capture sunlight efficiently during the day and use this during the night. Furthermore it allows them to perform light sensitive processes (such as cell division) at night. However, starch is considered as an unwanted product for TAG production, as starch and TAG compete for C3 precursors. With this in mind, starchless mutants have been created. Blocking the ability to make starch results in an higher TAG yield on light under batch nitrogen starvation conditions. However, it also results in reduced growth under nitrogen replete conditions compared to the wild-type.

The focus of this thesis is on understanding the role of starch in the oleaginous microalga Tetradesmus obliquus, a promising candidate for lipid production. In order to look into the role of starch, the previously developed starchless mutant of T. obliquus s/m1 was studied under different conditions and compared to the performance of the wild-type.

First, the role of starch was examined under nitrogen replete conditions (growth phase). Under these conditions the effect on cell physiology of LD cycles compared to continuous light was studied in Chapter 2. Here, the benefit of synchronization of metabolism to LD cycles was clear, as the wild-type utilized light $13 \%$ more efficiency under 16:8 h LD cycles compared to under continuous light. Concomitant with the 
synchronization to the LD cycles, cell composition changed throughout the diurnal cycle. Starch acted as a transitory diurnal energy storage compound in T. obliquus wildtype. It was accumulated during the late part of the light period and was consumed during the dark period and the following first hours of the light period. When looking into the behaviour of the starchless mutant $\operatorname{s} / m 1$, no other compound took over the role of starch as a transitory energy storage compound. Furthermore reduced growth was observed in the starchless mutant, compared to the wild-type, under both continuous light and LD cycles. Additionally, the benefit of the LD cycles compared to continuous light, as observed for the wild-type, was lost, as the energy utilization efficiency remained equal under both light regimes for the $\operatorname{s} / m 1$. These findings also showed that there are more benefits in accumulating starch on top of being a carbon and energy source during the dark periods, otherwise the energy efficiency of the $s / m 1$ would have remained equal to the wild-type under continuous light.

Next, we looked into the timing of cellular processes by studying the diurnal changes in the transcriptome profile of both $T$. obliquus wild-type and $\operatorname{s} / m 1$ under 16:8 h LD cycles in Chapter 3. RNA samples from turbidostat controlled experiments were analysed in intervals of one hour for the wild-type (for a high resolution) and three hours for the s/m1. For the wild-type, 4686 genes (23\% of all genes identified) were determined to have a significant change of expression over the diurnal cycle. These genes were classified into six clusters, whose expression peaked at different times of the diurnal cycle. Starting from the onset of light, these clusters captured enriched biological processes that occurred over time throughout the organism (e.g. expression of cluster 6 is maximum at 3 hours after light is on, and decreases until 10 hours after light on. Processes in this cluster revolve around the photosystem, including chlorophyll synthesis. The results from the transcriptomic analysis can also be correlated to the observations in Chapter 2. For example, the synthesis of both chlorophyll and carotenoids directly correlates to the dilution rate. Also, the increase in cell size during the day matches the expression profile of processes related to cell growth, with a high expression during most of the light period. When comparing the profile of the $\operatorname{sim} 1$ to the one from the wild-type, the majority of the genes with a different expression in time showed a time shift in expression. In addition, for some genes the profile changed completely. 
In the case of outdoor production with microalgae, variations in the length of the day and night periods will occur naturally. Therefore, in Chapter 4 we compared the impact of three different LD cycles (12:12 h, 14:10 h, and 16:8 h LD) on T. obliquus wild-type and starchless mutant s/m1. For the wild-type, the maximum measured content was reached when the night started and longer light periods resulted in an higher starch content. $\left(0.22 \mathrm{~g} \cdot \mathrm{g}_{D W^{-1}}\right.$ for the $16: 8 \mathrm{~h} \mathrm{LD}, 0.18 \mathrm{~g} \cdot \mathrm{g}_{D W^{-1}}$ for the $14: 10 \mathrm{~h}$ LD and $0.16 \mathrm{~g} \cdot \mathrm{g}_{D W^{-}}$ ${ }^{1}$ for the 12:2 h LD cycle). Additionally, starch was not fully consumed during the dark period and a fraction remained (0.03-0.06 $\left.\mathrm{g} \cdot \mathrm{g}_{\mathrm{DW}}{ }^{-1}\right)$, indicating that only $70-80 \%$ of the reserve is needed during the dark period, independently of its length. For the wild-type and the $\operatorname{sim} 1$ mutant the start of cell division was independent of the length of the photoperiod. However, cell division started earlier for the mutant (10-12 $\mathrm{h}$ after the light went on) than for the wild type ( $14 \mathrm{~h}$ after the beginning of the light period). Overall, the s/m1 mutant showed a lower photosynthetic efficiency compared to the wild-type, with the 12:12 h LD resulting into even less efficiency than the other two LD cycles.

After studying the role of starch under nitrogen replete conditions (growth phase), we continued by looking into the role of starch under nitrogen limitation (TAG production phase) in Chapter 5. During nitrogen limitation, starch continued to be the preferred storage compound for the wild-type to store energy and carbon. During the diurnal cycle, starch was accumulated to a maximum average content of $0.25 \mathrm{~g} \cdot \mathrm{gDw}^{-1}$, which is higher than the maximum observed under nitrogen replete conditions. Furthermore, small oscillations were observed, indicating that starch was still being used as a diurnal energy storage compound, but to a lesser extent than under nitrogen replete conditions. For the s/m1 mutant, the TAG content was higher than for the wild-type. However, despite the higher TAG content, we found that the photosynthetic efficiency was lower for the $\operatorname{s} / m 1$ mutant compared to the wild-type, especially during the second half of the light period, where starch accumulation occurred in the wild-type.

Metabolic models can help to get a better understanding of metabolism. With this in mind, we developed a metabolic model for the core metabolism of T. obliquus in Chapter 6. The network included 351 reactions with 183 metabolites distributed over 4 compartments: cytosol, chloroplast, mitochondria, and extracellular space. The energy requirements for biomass assembly and maintenance ( $K_{x}$ and $m_{A T P}$, respectively) were experimentally determined. A common strategy to estimate the 
energy parameters is using a chemostat set-up. However, we demonstrated the successful use of batch cultures to estimate these parameter in microalgae. This is because the light-limited growth in batch cultures allows to go slowly through different specific growth rates throughout the cultivation. The determined values were $121.02 \mathrm{mmol}_{A T P} \cdot g_{D W^{-1}}$ for $K_{x}$ and $0.66 \mathrm{mmol}_{\text {ATP }} \cdot g_{D W^{-1}} \cdot h^{-1}$ for the $m_{A T P}$. Based on the model the theoretical maximum yields for biomass, triacylglycerides (TAG), and starch yield on light were calculated to be $1.15 \mathrm{~g} \cdot \mathrm{mol}_{\mathrm{ph}}{ }^{-1}, 1.05 \mathrm{~g}_{\mathrm{TAG}} \cdot \mathrm{mol}_{\mathrm{ph}}{ }^{-1}$, and $2.69 \mathrm{~g}_{\mathrm{starch}} \cdot \mathrm{mol}_{\mathrm{ph}}{ }^{-1}$.

Finally, in Chapter 7 the role of starch was discussed based on the findings in the previous chapters. The role of starch depends on the environmental conditions. First, under nitrogen replete conditions (growth phase) starch serves as a short-term diurnal energy storage compound that can be easily used during the dark period. During the dark period, starch is used for processes such as cell division and nitrogen fixation. Additionally, starch is also consumed at the beginning of the light period when photosynthesis is active, possibly supplying extra energy to rapidly produce pigments for the harvesting of light energy. Under nitrogen limitation, starch still functions as a diurnal energy storage compound, but to a lesser extent. Finally, under nitrogen starvation, starch is the preferred storage compound for energy and carbon in T. obliquus wild-type, but this is no longer used as a diurnal energy storage compound. Instead, starch serves as a storage compound to be used for recovery and as an electron sink. TAG accumulation occurs once the starch storage capacity is "full" (approximately $0.40 \mathrm{~g} \cdot \mathrm{g}_{\mathrm{DW}}{ }^{-1}$ ). Moreover, when nitrogen remains depleted, starch is also converted to TAG, and TAG serves as a long term storage compound to be used for recovery when nitrogen is again available. 


\section{Resumen}

Las microalgas son una materia prima de gran potencial para la producción de biocombustibles, alimentos, y compuestos de valor agregado. En particular gracias a su capacidad de acumular lípidos. Las microalgas oleaginosas pueden alcanzar un contenido de triacilglicéridos (TAG) de hasta un $60 \%$ de su peso seco bajo condiciones de estrés. En contraste con las plantas oleaginosas, tienen una mayor productividad por unidad de área y no compiten por suelos cultivables.

La producción de microalgas a gran escala se divide comúnmente en dos fases: la fase de crecimiento y la de producción de lípidos (TAG). Durante la fase de crecimiento, se utilizan condiciones favorables de crecimiento en las cuales se provee nitrógeno en exceso, mientras que para la producción de lípidos se utilizan condiciones de limitación de nitrógeno. Muchos microorganismos fotosintéticos sincronizan su metabolismo durante la fase de crecimiento acorde con los ciclos de luz y oscuridad (L/O). Durante estos ciclos muchas microalgas acumulan energía luminosa y carbono en forma de almidón. De este modo, éstas capturan la luz solar de forma eficiente durante el día y utilizan las reservas durante la noche, permitiéndoles además realizar procesos fotosensibles (por ejemplo la división celular) en la oscuridad. No obstante, la síntesis de almidón es indeseable para la producción de TAG, ya que ambos compuestos compiten por precursores de tres carbonos. Con esto en mente, se han cepas microalgales incapaces de producir almidón, lo cual ha resultado en un mayor rendimiento lipídico bajo condiciones de inanición celular de nitrógeno. Sin embargo, el crecimiento de estas cepas es menor bajo condiciones de suficiencia de nitrógeno comparado con la cepa parental (silvestre).

El objetivo de esta tesis es el de entender el rol del almidón en la fisiología y la producción lipídica de la microalga oleaginosa Tetradesmus obliquus, la cual es una especie de interés para la producción de lípidos. Para este propósito, realizamos un estudio comparativo entre la cepa $T$. obliquus s/m1, incapaz de producir almidón, y su cepa parental. El comportamiento de ambas cepas se evaluó bajo diferentes condiciones de cultivo. 
Primeramente, estudiamos el rol del almidón bajo condiciones de suficiencia de nitrógeno (fase de crecimiento). Bajo estas condiciones, examinamos el efecto de ciclos de L/O comparado con el uso de luz continua en la fisiología celular de $T$. obliquus (Capítulo 2). Aquí, se observaron claramente los beneficios de la sincronización del metabolismo a los ciclos L/O, ya que la cepa parental utilizó la energía luminosa 13\% más eficientemente bajo ciclos de 16:8 h L/O comparado con luz continua. Simultáneamente con la sincronización a los ciclos L/O, la composición celular cambió durante el ciclo diurno. El almidón es un compuesto diurno transitorio de almacenamiento de energía en $T$. obliquus, siendo acumulado durante la última parte del período de luz y posteriormente consumido durante el período de oscuridad y las siguientes horas de luz. En la cepa mutante $s / m 1$, ningún otro compuesto adicional reemplazó el rol del almidón como almacenamiento transitorio de energía. En contraste con la cepa parental, el crecimiento en $\operatorname{s} / m 1$ fue menor bajo luz continua y los ciclos L/O. Adicionalmente, el beneficio observado durante los ciclos L/O comparado con luz continua se perdió, ya que la eficiencia de uso de energía permaneció constante durante ambas condiciones en $s / m 1$. Estos resultados demuestran que la acumulación de almidón está relacionada con procesos fisiológicos adicionales a su uso como fuente de carbono y energía durante los períodos de oscuridad, de lo contrario la eficiencia energética de $\operatorname{s} / m 1$ hubiera permanecido igual a la de su cepa parental bajo condiciones de luz continua.

A continuación, estudiamos la regulación de los procesos celulares durante el ciclo diurno mediante el análisis transcriptómico de T. obliquus bajo ciclos de 16:8 h L/O (Capítulo 3). Muestras de ARN provenientes de cultivos turbidostato se colectaron en intervalos de una y tres horas para la cepa parental (para alta resolución) y cepa mutante $s / m 1$, respectivamente. En la cepa parental, 4686 genes (23\% de todos los identificados) mostraron un cambio significativo en su expresión durante el ciclo diurno. Estos genes fueron clasificados en seis grupos (clusters), acorde con su perfil de expresión durante el ciclo diurno, alcanzando su punto máximo a diferentes horas del día. Comenzando por el momento en el que la luz se enciende, estos grupos incluyen enriquecimientos de procesos biológicos que ocurren durante el ciclo diurno en el organismo (por ejemplo, la expresión del grupo 6 alcanza su máximo 3 horas después de que la luz se enciende, y disminuye gradualmente hasta las 10 horas). Los procesos en este grupo giran en torno al fotosistema, incluyendo la síntesis de clorofila. 
Los resultados del análisis transcriptómico son consistentes con las observaciones del análisis descrito en el Capítulo 2. Por ejemplo, la expresión de genes relaciones con la síntesis de los pigmentos clorofila y carotenoides directamente correlaciona con la tasa de dilución. Asimismo, el incremento observado en el tamaño celular durante el día coincidió con el perfil de expresión de genes relacionados con el crecimiento celular, con una expresión alta durante la mayor parte del período de luz. Al comparar el perfil transcriptómico de la cepa mutante $\operatorname{s} / m 1$ con el de la cepa parental, la mayoría de los genes con una diferencia en la expresión muestran un cambio de momento en la expresión, aunque el perfil transcriptómico de algunos genes cambió drásticamente.

La producción de microalgas a gran escala en exteriores está supeditada a las variaciones naturales en la duración del día y la noche. Basándonos en esto, en el Capítulo 4 comparamos el efecto de tres diferentes ciclos L/O (12:12 h, 14:10 h, y 16:8 h L/O) en T. obliquus. En la cepa parental, el contenido máximo se alcanzó al inicio de la noche y los periodos de luz más largos resultaron en una contenido más alto de almidón (en peso seco, PS: $0.22 \mathrm{~g} \cdot \mathrm{gPs}^{-1}, 16: 8 \mathrm{~h} \mathrm{L/O}$; $0.18 \mathrm{~g} \cdot \mathrm{gPS}^{-1}, 14: 10 \mathrm{~h} \mathrm{~L} / 0$; y 0.16 g. Ps $^{-1}, 12: 2 \mathrm{~h}$ L/O). Adicionalmente, el almidón nunca fue consumido totalmente durante el período de oscuridad, quedando una fracción (0.03-0.06 g. $\left.\mathrm{gPs}^{-1}\right)$, indicando que sólo el $70-80 \%$ de las reservas de almidón son necesarias durante el periodo de oscuridad, independientemente de su duración. Para ambas cepas, el inicio de la división celular fue independiente del fotoperiodo. Sin embargo, la división celular inició más temprano para la mutante (10-12 h después del encendido de la luz) comparada con la cepa parental (14 h después del encendido de la luz). En general, la mutante s/m1 mostró una menor eficiencia fotosintética comparada con la cepa parental, particularmente en el ciclo con el periodo de luz más corto (12:12 h L/O).

Después de estudiar el rol del almidón bajo condiciones de suficiencia de nitrógeno (fase de crecimiento), investigamos el rol del almidón bajo condiciones de limitación de nitrógeno (fase de producción de lípidos, TAG) en el Capítulo 5. Bajo condiciones de limitación de nitrógeno, el almidón continuó siendo el compuesto preferido para almacenar energía y carbono en la cepa parental. Durante el ciclo diurno, el almidón fue acumulado hasta un convenido promedio máximo de $0.25 \mathrm{~g} \cdot \mathrm{gPs}^{-1}$, el cual fue mayor al contenido máximo observado en condiciones de suficiencia de nitrógeno. Asimismo, observamos pequeñas oscilaciones en el contenido de almidón, indicando que éste funciona como compuesto diurno de almacenamiento de energía, pero en menor 
grado que bajo condiciones de suficiencia de nitrógeno. El contenido de TAG fue más alto en la mutante s/m1 comparada con la cepa parental. Sin embargo, la eficiencia fotosintética de la cepa mutante fue menor, especialmente durante la segunda mitad del periodo de luz, etapa en la cual el almidón fue acumulado en la cepa parental.

Los modelos metabólicos permiten un mejor entendimiento de la fisiología de un organismo. Con esto en mente, desarrollamos un modelo para el metabolismo central de T. obliquus en el Capítulo 6. El modelo incluye 351 reacciones con 183 metabolitos distribuidos en cuatro compartimentos: citosol, cloroplasto, microcondria, y espacio extracelular. Los requerimientos energéticos para la producción de biomasa y mantenimiento celular ( $K_{x} \quad y \quad m_{A T P}$, respectivamente) fueron determinados experimentalmente. A pesar de que estos parámetros energéticos son comúnmente estimados mediante el uso de un quimiostato, en este trabajo demostramos el uso exitoso de cultivos en lote (batch) para este propósito. El cálculo de estos parámetros es posible mediante esta estrategia porque el crecimiento limitado por luz en cultivos microalgales tipo lote permite ir lentamente a través de diferentes tasas de crecimiento específicas durante todo el cultivo. Los valores determinados fueron $121.02 \mathrm{mmol}_{A T P} \cdot \mathrm{gPS}^{-1}$ para $K_{\mathrm{x}}$ y $0.66 \mathrm{mmol}_{\text {ATP }} \cdot \mathrm{g}_{\mathrm{PS}}{ }^{-1} \cdot \mathrm{h}^{-1}$ para $\mathrm{m}_{\text {ATP. }}$ Los rendimientos teóricos máximos calculados en base en el modelo fueron: biomasa, $1.15 \mathrm{~g} \cdot \mathrm{mol}_{\text {fotones }}{ }^{-1}$, TAG, $1.05 \mathrm{~g}_{\mathrm{TAG}} \cdot \mathrm{mol}_{\text {fotones }}{ }^{-1}$, y almidón, $2.69 \mathrm{~g}_{\text {almidón }} \cdot \mathrm{mol}_{\text {fotones }}{ }^{-1}$.

Finalmente, en el Capítulo 7 se discute el rol del almidón con base en los resultados descritos en los capítulos anteriores. La función del almidón depende de las condiciones ambientales. Primero, bajo condiciones de suficiencia de nitrógeno (fase de crecimiento) el almidón sirve como un compuesto diurno de almacenamiento de energía a corto plazo que puede ser fácilmente utilizado durante el periodo de oscuridad, en el cual es utilizado para procesos como la división celular y la fijación de nitrógeno. Adicionalmente, el almidón es catabolizado durante el periodo de luz, cuando la fotosíntesis está activa, probablemente suministrando energía para la producción inmediata de pigmentos para la recolección de energía luminosa. Bajo condiciones de limitación de nitrógeno, el almidón aún funciona como un compuesto diurno de almacenamiento de energía, pero en un menor grado. Finalmente, bajo condiciones de inanición celular de nitrógeno, el almidón es el compuesto preferido para almacenar energía y carbono en la cepa silvestre de T. obliquus, pero no es utilizado como compuesto diurno de almacenamiento. En su lugar, el almidón funciona 
como sumidero de electrones, así como un compuesto temporal de reserva para la posterior recuperación de la microalga. La acumulación de lípidos (TAG) ocurren una vez que la capacidad de almacenamiento de almidón ha sido saturada (aprox.

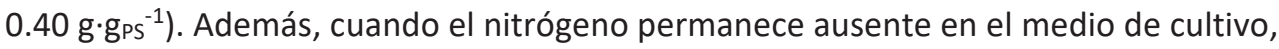
el almidón también es convertido a TAG, fungiendo éste como un compuesto de almacenamiento a largo plazo que será utilizado para la recuperación una vez que el nitrógeno vuelve a estar disponible. 


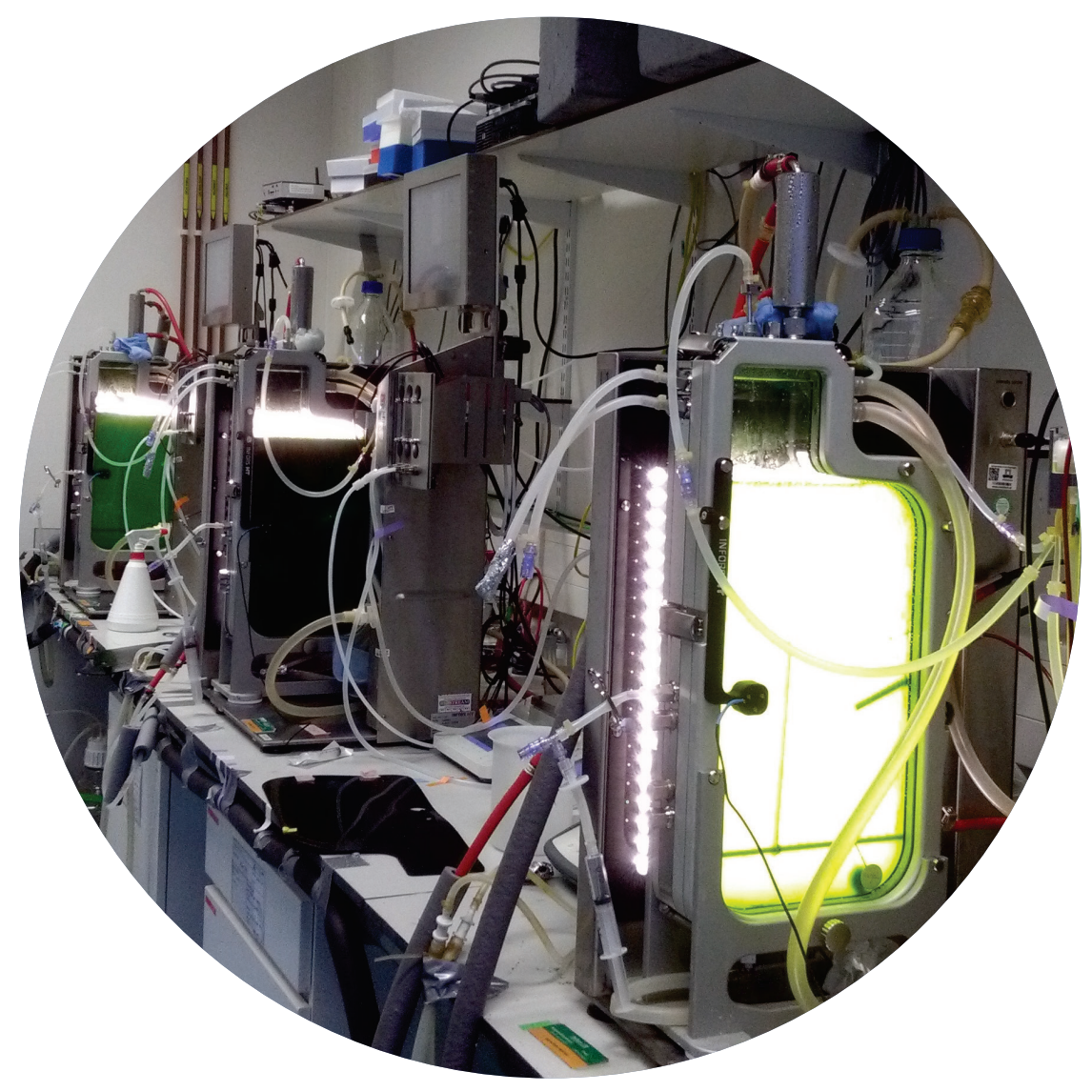


Nomenclature 
$\mu$

$\mu_{\mathrm{t}}$

$\mathrm{C}_{\text {cells }}$

CET

$\mathrm{CHO}$

$\mathrm{CL}$

$D_{24 h}$

$D_{t}$

DW

FBA

GO

$\mathrm{K}_{\mathrm{x}}$

LD

L/O

LET

$\mathrm{m}_{\text {ATP }}$

$\mathrm{mol}_{\mathrm{ph}}$

NSC

OD

PC

$\mathrm{Ph}$

$r_{p h}$

$r_{\text {starch, } t}$

slm1

$\mathrm{t}$

$t_{d}$

TAG

TES

TFA

$V_{P B R}$

$Y_{T A G, p h}$

$Y_{\mathrm{x}, \mathrm{ph}}$
Growth rate

Time-specific cell division rate

Concentration of cells

Cyclic electron transport

Carbohydrates

Continuous light

Average dilution rate over $24 \mathrm{~h}$

Dilution rate over small time intervals

Dry weight

Flux Balance Analysis

Gene Ontology

Growth associated maintenance

Light/dark

Luz/oscuridad

Linear electron transport

non-growth associated maintenance

$$
\text { mol of photons }
$$

Non-starch carbohydrates

Optical density

Principal component

Photons

volumetric photon supply rate

Starch productivity

Starchless mutant 1

Time

Doubling time

Triacylglycerides

Transitory energy storage

Total fatty acids

Photobioreactor volume

Triacylglycerol yield on light

Biomass yield on light 


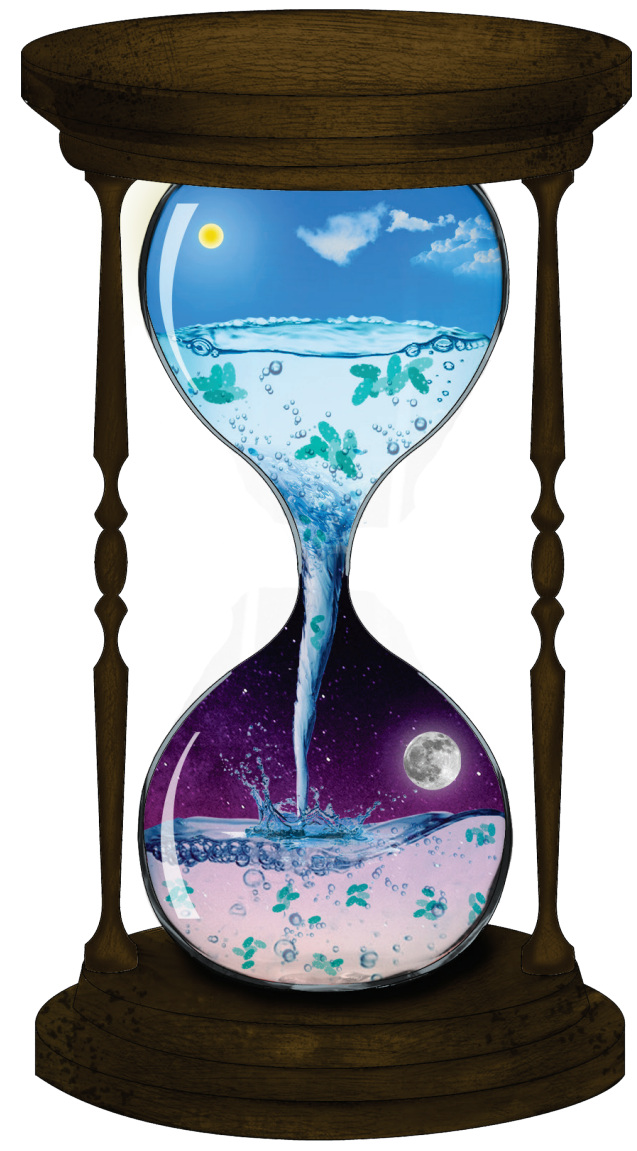


Acknowledgements

Agradecimientos 
I would like to thank all the people who were part of my PhD during the past four years. First or all, I want to thank my supervisors for their guidance. René, thank you for all the useful input during the meetings and for giving me the opportunity to be part of the BPE family for my PhD. Douwe, it was really enjoyable to work with you from day one. Thanks for, amongst others, supporting all the "crazy" ideas for my project, for introducing me into the Dutch culture, and for all the useful meetings and nice coffee breaks. Dirk, thank you for your daily supervision, for always making time to discuss, read and answer my questions (either in person or on skype).

Next I want to thank all the technicians, because all PhDs need technical support. Snežana, "angel", thanks for the motivation and the good talks, you always had a moment to check on us. Wendy, thank you for the help with all the analytical methods and equipment and for always making time to answer my questions. Fred, thanks for all the help with the reactors, the autoclave and "iris", the last two for sure gave me quite some problems. Sebastiaan, even though my shoes never arrived, thanks for your help with the reactors.

Also, thanks to Miranda and Marina for all the administrative help. Specially, Miranda thanks for your help since the day I started my application to come to the Netherlands! I would like to thank the members of the BPE team for all the good chats, coffee breaks, borrels, labuitjes, and Christmas dinners: Ági, Anne, Arjen, Azis, Brenda, Camilo, Catalina, Christian, Corjan, Dorinde, Douwe, Edgar, Edwin, Elisa, Enrico, Fabian, Gerard, Gosia, Hans, Iago, Ilse, João, Jorijn, Kim, Kylie, Lenny, Luci, Marcel, Maria, Marian, Marjon, Mark, Marta, Michel, Narcís, Packo, Pauline, Pieter, Rafa, Renske, Richard, Rouke, Ruchir, Rupali, Ruud, Stephanie, Ward, Xiao, Youri. Arjen, thanks for always cheering us up in the office (and for bringing candy for us). I would like to give a special "thank you" to the members of the "sexy girl office": Ilse, Marjon, Edwin, Edgar, and Christian. It was a real pleasure sharing the office with all of you, you made the time in the office much more enjoyable!

Thanks to all the students who were always enthusiastic and motivated, and never complained about coming to the lab on the weekends: Seyi, Sidrah, Narcís, Davide, David y Tània.

To my international friends: Ági, Gerard, Ilse, Imma, Jorijn, Marta, Paulo (and Jo), Rupali, Xiao (and Yimin). Thanks for all the nice dinners, cooking was never so fun! and 
I hope we can meet again soon! Rupali, you made the lunch and coffee breaks more lively and fun (though Douwe probably didn't enjoy them as much). Ági, it was always very fun to have you around, let's see where we meet next (knowing you, it could be anywhere in the world, maybe Mexico?). Gerard, I had a lot of nice talks with you, somehow you could always find a nice conversation topic to keep the coffee breaks alive. Imma, even though your time in Wageningen was not so long, we became really good friends. Thanks for being there for me to talk and hope to see you in my defence! Marta, thanks for all the good times and food (Natas), it's been really nice to share so many happy moments (including meeting little Jo). Xiao and Yimin, thanks for showing us a bit of your culture, I had a lot of fun with the cooking and the tea workshop, I hope to meet Muyun soon!

To my lovely Paranymphs, I am really happy that you both are joining me on stage (additionally to have joined you both there)! Ilse, you were like my PhD sister, we shared the office since the day I started and we even wrote a paper together! Thanks for all the support, and for always being someone I could talk to (about work and nonwork related topics). Jorijn, I was really surprised and happy when Ilse and you joined me on my trip to Mexico. I had a great time during that and all the other trips we made together. Thanks for the constant support, for the nice dinners and tea evenings where I could share my frustrations and joys. You even made the gym a bit less annoying for me (gezellig?).

Thanks to all the nice people I met in Wageningen during these 4 years! Ale, me dio mucho gusto conocerte por estos rumbos y, aunque no estuvimos mucho tiempo en el mismo país, siempre podíamos platicar de todo. Vera and James ("Mr. President"), thanks for all the fun moments we shared while working in the same team.

A todos mis amigos dentro y fuera de México, gracias por, de una u otra forma, apoyarme sin importar la distancia. Especialmente, igracias Paul por ayudarme con el diseño de la portada! Adriana, gracias por introducirme al mundo de las microalgas y por ayudarme a corregir el resumen.

Lily, our friendship started a few years ago in Canada and we somehow have managed to stay in touch (for almost 10 years). I was really happy to see you again in the Netherlands, Germany, and France, and I hope we can meet again soon (Christmas markets?). 
Quiero agradecer a mi familia, por alegrarme cada día con fotos, mensajes, y llamadas. Papá, mamá, Sashi (Juan, Sayuri y Satoru), Hiro (y Brenda), gracias por su apoyo incondicional. Gracias también a tía Chela, tío Take y Tía Lupita, tía Nelly, tía Tere y tío Felipe, Take y Kohji. Katzuo, nos dejaste cuando apenas empezaba esta etapa y seguimos extrañándote como el primer día.

Für meine neue Familie, Helena und Hubert. Ihr habt mich seit dem ersten Tag in eurem Haus willkommen geheißen. Danke, dass ihr meine Familie weit weg von zu Hause seid. Dasselbe gilt für Jola, Marta, Ela und Zygmunt, danke, dass ihr mich so herzlich aufgenommen habt.

Finally, my dear Martin, my better half. You joined me in the middle of my journey through my PhD and we have been walking together ever since (although you actually have been in my life before I started). Danke, dass du für mich immer da bist, für deine Motivation, Unterstützung und dein Verständnis. I do not know how I would have done it without you! 


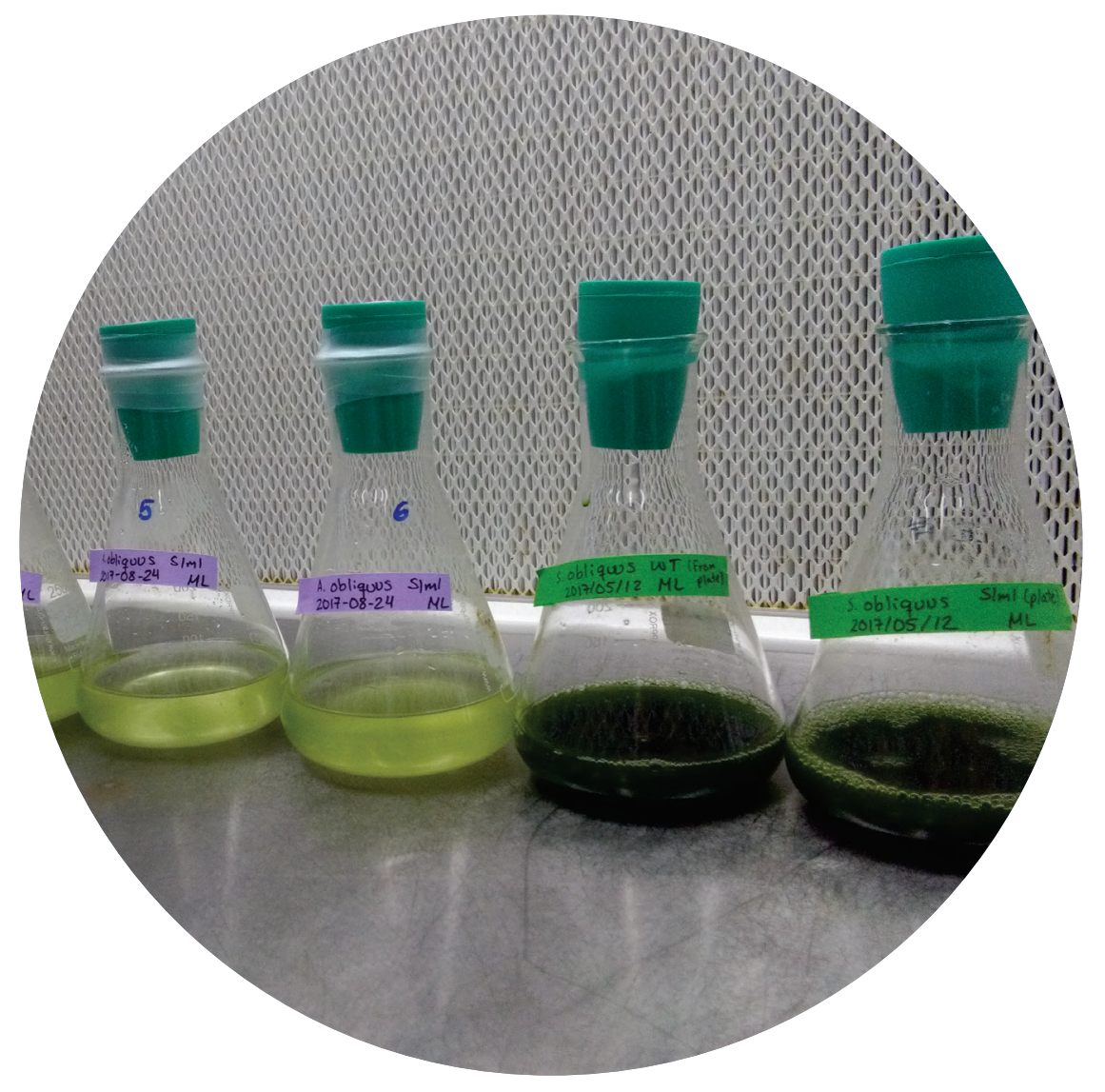


About the author 


\section{Curriculum Vitae}

Graciela Mitsue León Saiki was born on September $23^{\text {rd }} 1986$ in Culiacán, México. She grew up and attended primary, secondary and high school in Culiacán.

In 2004, she started her studies at the Faculty of Biological and Chemical Sciences from the Autonomous University of Sinaloa (Universidad Autónoma de Sinaloa). During her Bachelor, she did a semester abroad at McGill University in Ste.-Anne-deBellevue, Canada. In 2009 she graduated with honours

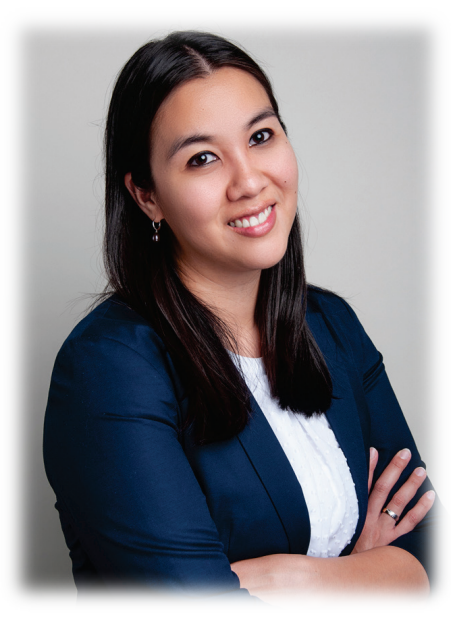
from her Bachelor in Biochemical Engineering. In 2010 she moved to Cuernavaca, México and she started her MSc in Biochemical Sciences at the Institute of Biotechnology of the Nacional Autonomous University of Mexico (Universidad Nacional Autónoma de México) where she started her research work on microalgae. For her MSc thesis she studied the effect of $\mathrm{CO}_{2}$ on growth and biochemical composition of the microalga Neochloris oleoabundans for its further use as a scale up criterion. During her MSc thesis, she visited the Institute of Biochemical Engineering from the Technical University of Braunschweig in Germany for an internship to conduct experimental work related to her thesis.

In 2013 she moved to Wageningen to start her doctorate (PhD) research in the Bioprocess Engineering group of Wageningen University and Research, studying the role of starch metabolism inside the microalga Tetradesmus obliquus. The results of her $\mathrm{PhD}$ research are described in this thesis. 


\section{List of publications}

GM León-Saiki*, IM Remmers*, DE Martens, PP Lamers, RH Wijffels, D van der Veen (2017).The role of starch as transient energy buffer in synchronized microalgal growth in Acutodesmus obliquus. Algal Research 25, 160-167.

* Authors contributed equally

GM León-Saiki, T Cabrero Martí, D van der Veen, RH Wijffels, DE Martens (2018). The impact of day length on cell division and efficiency of light use in a starchless mutant of Tetradesmus obliquus. Algal Research 31, 387-394

GM León-Saiki, N Ferrer Ledo, D Lao-Martil, D van der Veen, RH Wijffels, DE Martens. Metabolic modelling and energy parameter estimation of Tetradesmus obliquus. Submitted for publication.

BM Carreres, GM León-Saiki, IM Remmers, D van der Veen, PJ Schaap, VAP Martins dos Santos, RH Wijffels, DE Martens, M Suarez-Diez. The diurnal transcriptional landscape of the microalga Tetradesmus obliquus. To be submitted for publication.

GM León-Saiki, IM Remmers, RH Wijffels, D van der Veen, DE Martens. Diurnal biochemical responses of Tetradesmus obliquus under light/dark cycles and nitrogen limitation. In preparation. 


\section{Overview of completed training activities}

\section{Discipline specific activities}

BPE Mini-symposium: 'Microalgae biotechnology: towards industrial

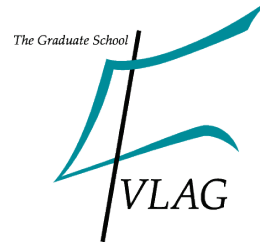

2013

strains and processes'

Biology of Plastids - Towards a Blueprint for Synthetic Organelles ${ }^{1}$

2013

2014

2014

NBC-15 Biotechnology by Dutch Design (Ede, The Netherlands) ${ }^{1}$

2014

PE\&PC Measuring the Photosynthetic Phenome ${ }^{1}$

2014

France $)^{1}$

VLAG/EPS Microscopy and Spectroscopy in Food and Plant Sciences

2014

VLAG Microalgae Process Design: from cells to photobioreactors

2014

BPE Mini-symposium: 'The dynamics of oil accumulation'

2015

Microbial stress: From Molecules to Systems (Sitges, Spain) ${ }^{1}$

2015

NBV/MSD Seminar 'Continuous processing for biotherapeutic proteins' VLAG Basic Statistics

2015

2016

BSDL Advance course Metabolomics for microbial system biology
2016

2013

2014

2015

2016

2016

2017

2013

2014

2014

2013-2016

${ }^{1}$ Poster ${ }^{2}$ Presentation 
This study was carried out at the department of Bioprocess Engineering of Wageningen University and Research, Wageningen, the Netherlands. The research described in this thesis was supported by the Consejo Nacional de Ciencia y Tecnología - CONACYT, Mexico, Scholar 218586/Scholarship 314173. In addition, GMLS is part of the program "Doctores Jóvenes para el Desarrollo Estratégico Institucional" by the Universidad Autónoma de Sinaloa.

This thesis was printed by DigiForce || ProefschriftMaken

Cover design by Paul Alejandro Pérez Ramos 
